COMMUNICATIONS IN

ANALYSIS AND GEOMETRY

Volume 4, Number 1, 1-120, 1996

\title{
Braid Group Technique in Complex Geometry V: The Fundamental Group of a Complement of a Branch Curve of a Veronese Generic Projection
}

\author{
Boris MoIShezon AND Mina TeICHER ${ }^{1}$
}

In this paper we compute $\pi_{1}\left(\mathbb{C}^{2}-S_{3}\right), S_{3}$ the branch curve of a generic projection of the Veronese surface $V_{3}$ on $\mathbb{C P}^{2}$. Throughout this paper we use $G$ for $\pi_{1}\left(\mathbb{C}^{2}-S_{3}\right)$. We also have a similar result concerning $\bar{G}=\pi_{1}\left(\mathbb{C P}^{2}-S_{3}\right)$.

Fundamental groups of complements of curves are very important invariants but it is very difficult to compute them. We obtained $G$ and $\bar{G}$ using our braid group techniques.

This paper is a continuation of "Braid Group Techniques in Complex Geometry, I, II, III, IV." (BGT I, BGT II, BGT III, BGT IV for short.) In BGT I we laid the foundation of our braid monodromy techniques and applied them to line arrangements. In BGT II we dealt with the braid monodromy of almost real curves and showed how to regenerate these formulas to cuspidal curves. In BGT III we presented a series of generic projected degenerations of the Veronese surface $V_{3}$ and the branch curve $S_{3}$ of its generic projection to $\mathbb{C P}^{2}$ to a union of 9 planes, and a branch curve $S^{(6)}$ which is a union of lines. In BGT IV we computed the braid monodromy of $S_{3} \cap \mathbb{C}^{2}$ using the braid monodromy of $S^{(6)}$ and the regeneration rules proved in BGT II. We obtained the factorized expression for the braid monodromy denoted by $\varepsilon(18)$. In this paper we use $\varepsilon(18)$ and the Van Kampen Theorem to compute $G$ and $\bar{G}$. If the reader is only interested in the final results he can go directly to Chapter VI.

\section{Definition of Braid Group and Presentation of the Van Kampen Theorem.}

We need certain terminology in order to formulate the Van Kampen Theorem.

\footnotetext{
${ }^{1}$ This research was partially supported by the Emmy Noether Mathematics Research Institute, Bar-Ilan University, Israel.
} 


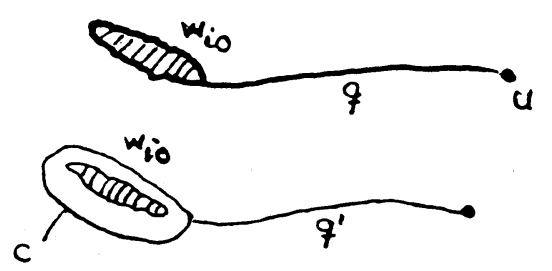

Figure 0.1.

We first recall from BGT I (Section II) the definition of a good geometric base of $\pi_{1}(D-K, *)$ for $K$ a finite set in a disc $D$.

Definition. $\ell(\gamma)$.

Let $D$ be a disc. Let $w_{i} i=1, \ldots, n$ small discs in $\operatorname{Int}(D)$ s.t. $w_{i} \cap w_{j}=\emptyset$ for $i \neq j$. Let $u \in \partial D$. Let $\gamma$ be a simple path connecting $u$ with one of the $w_{i}$ 's, say $w_{i_{0}}$, which does not meet any other $w_{j}, j \neq i_{0}$. We assign to $\gamma$ a loop (actually an element of $\pi_{1}(D-K, u)$ ) as follows. Let $c$ be a simple loop equal to the (oriented) boundary of a small neighborhood $V$ of $w_{i_{0}}$ chosen such that $\gamma^{\prime}=\gamma-V \cap \gamma$ is a simple path. Then $\ell(\gamma)=\gamma^{\prime} \cup c \cup \gamma^{\prime-1}$. We also use the same notation $\ell(\gamma)$ for the element of $\pi_{1}(D-K, u)$ corresponding to $\ell(\gamma)$ (see Fig. 0.1).

Definition. $\quad g$-base (good geometric base)

Let $D$ be a disk, $K \subseteq D, \# K<\infty$. Let $u \in D-K$. Let $\left\{\gamma_{i}\right\}$ be a bush in $(D, K, u)$, i.e., $\forall i, j \gamma_{i} \cap \gamma_{j}=u, \forall i \gamma_{i} \cap K=$ one point, and $\gamma_{i}$ are ordered counterclockwise around $u$. Let $\Gamma_{i}=\ell\left(\gamma_{i}\right) \in \pi_{1}(D-K, u)$ be the loop around $K \cap \gamma_{i}$ determined by $\gamma_{i} .\left\{\Gamma_{i}\right\}$ is a $g$-base of $\pi_{1}(D-K, *)$.

Definition. Braid group $B_{n}=B_{n}[D, K]$

Let $D$ be a closed disc in $\mathbb{R}^{2}, K \subset D, K$ finite. Let $B$ be the group of all diffeomorphisms $\beta$ of $D$ such that $\beta(K)=K,\left.\beta\right|_{\partial D}=\operatorname{Id}_{\partial D}$. For $\beta_{1}, \beta_{2} \in B$, we say that $\beta_{1}$ is equivalent to $\beta_{2}$ if $\beta_{1}$ and $\beta_{2}$ induce the same automorphism of $\pi_{1}(D-K, u)$. The quotient of $B$ by this equivalence relation is called the braid group $B_{n}[D, K](n=\# K)$. We sometimes denote by $\bar{\beta}$ the braid represented by $\beta$. The elements of $B_{n}[D, K]$ are called braids.

Definition. $H(\sigma)$, half-twist defined by $\sigma$ 
Let $D, K$ be as above. Let $a, b \in K, K_{a, b}=K-a-b$ and $\sigma$ be a simple path in $D-\partial D$ connecting $a$ with $b$ s.t. $\sigma \cap K=\{a, b\}$. Choose a small regular neighborhood $U$ of $\sigma$ and an orientation preserving diffeomorphism $f: \mathbb{R}^{2} \rightarrow \mathbb{C}^{1}\left(\mathbb{C}^{1}\right.$ is taken with the usual "complex" orientation) such that $f(\sigma)=[-1,1], \quad f(U)=\left\{z \in \mathbb{C}^{1}|| z \mid<2\right\}$. Let $\alpha(r), r \geq 0$, be a real smooth monotone function such that $\alpha(r)=1$ for $r \in\left[0, \frac{3}{2}\right]$ and $\alpha(r)=0$ for $r \geq 2$.

Define a diffeomorphism $h: \mathbb{C}^{1} \longrightarrow \mathbb{C}^{1}$ as follows. For $z \in \mathbb{C}^{1}, z=r e^{i \varphi}$, let $h(z)=r e^{i(\varphi+\alpha(r))}$. It is clear that on $\left\{z \in \mathbb{C}^{1}|| z \mid \leq \frac{3}{2}\right\}, h(z)$ is the positive rotation by $180^{\circ}$ and that $h(z)=$ Identity on $\left\{z \in \mathbb{C}^{1}|| z \mid \geq 2\right\}$, in particular, on $\mathbb{C}^{1}-f(U)$. Considering $\left.\left(f \circ h \circ f^{-1}\right)\right|_{D}$ (we always take composition from left to right), we get a diffeomorphism of $D$ which switches $a$ and $b$ and is the identity on $D-U$. Thus it defines an element of $B_{n}[D, K]$, called the half-twist defined by $\sigma$ and denoted $H(\sigma)$.

Definition. Frame of $B_{n}[D, K]$

Let $D$ be a disc in $\mathbb{R}^{2}$. Let $K=\left\{a_{1}, \ldots, a_{n}\right\}, K \subset D$. Let $\sigma_{1}, \ldots, \sigma_{n-1}$ be a system of simple paths in $D-\partial D$ such that each $\sigma_{i}$ connects $a_{i}$ with $a_{i+1}$ and for

$$
i, j \in\{1, \ldots, n-1\}, i<j \quad, \quad \sigma_{i} \cap \sigma_{j}= \begin{cases}\emptyset & \text { if }|i-j| \geq 2 \\ a_{i+1} & \text { if } j=i+1 .\end{cases}
$$

Let $H_{i}=H\left(\sigma_{i}\right)$. We call the ordered system of (positive) half-twists $\left(H_{1}, \ldots, H_{n-1}\right)$ a frame of $B_{n}[D, K]$ defined by $\left(\sigma_{1}, \ldots, \sigma_{n-1}\right)$, or a frame of $B_{n}[D, K]$ for short.

\section{Notation.}

$$
\begin{aligned}
& {[A, B]=A B A^{-1} B^{-1} .} \\
& \langle A, B\rangle=A B A B^{-1} A^{-1} B^{-1} . \\
& (A)_{B}=B^{-1} A B .
\end{aligned}
$$

Theorem (E. Artin's braid group presentation). $B_{n}$ is generated by the half-twists $H_{i}$ of a frame $\left\{H_{i}\right\}$ and all the relations between $H_{1}, \ldots, H_{n-1}$ follow from

$$
\begin{gathered}
{\left[H_{i}, H_{j}\right]=1 \quad \text { if } \quad|i-j|>1} \\
\left\langle H_{i}, H_{j}\right\rangle=1 \quad \text { if } \quad|i-j|=1 \\
1 \leq i, j \leq n-1 .
\end{gathered}
$$


Proof. See proof in [MoTe4], Chapter 4.

Proposition-Definition. $\Delta_{n}^{2}\left(\in B_{n}\right)$

$\Delta_{n}^{2}=\left(H_{1} \ldots H_{n}\right)^{n-1}$ for any frame $H_{1} \ldots H_{n-1}$ of $B_{n}$.

We shall need the following definition:

Definition. $R(\varepsilon), N(\varepsilon), G(\varepsilon)$ where $\varepsilon$ is a factorized expression in $B_{m}$

Let $D$ be a disk in $\mathbb{C}, K \subseteq D, \# K=m$.

Let $\mathbb{F}_{m}=\pi_{1}(D-K)$. Consider the natural action of $B_{m}=B[D, K]$ on $\mathbb{F}_{m}$, denoted by $(\Gamma) g_{i}$.

Let $\varepsilon=g_{1} \cdots g_{t}$ be a factorized expression in $B_{m}$.

Let $\Gamma_{1} \cdots \Gamma_{m}$ be a good geometric base of $\mathbb{F}_{m}$.

Let $M(\varepsilon)$ be the subgroup of $B_{m}$ generated by $\left\{g_{i}\right\}_{i=1}^{t}$.

Let $R(\varepsilon)$ be the subgroup of $\mathbb{F}_{m}$ generated by $\left\{\left(\Gamma_{j}\right) g_{i} \circ \Gamma_{j}^{-1}\right\}_{i=1, j=1}^{t}$.

Let $N(\varepsilon)$ be the normal subgroup of $\mathbb{F}_{m}$ generated by $R(\varepsilon)$.

Let $G(\varepsilon)=\frac{\mathbb{F}_{m}}{N(\varepsilon)}$.

Lemma 0.1. $\quad$ (i) $N(\varepsilon)=\left\{(\alpha) \beta \cdot \alpha^{-1} \mid \alpha \in \mathbb{F}_{m}, \quad \beta \in M(\varepsilon)\right\}$.

(ii) $G(\varepsilon)=F_{m}$ with the relations induced from $R(\varepsilon)$.

Proof. Trivial.

We recall from BGT IV [MoTe7] the definitions of Hurwitz equivalent factorizations and factorization invariant under $h \in B_{m}$.

Definition. Hurwitz move

Let $g_{i} \cdots \cdots g_{k}=h_{i} \cdots \cdots h_{k}$ be two factorized expressions of the same element in a group $G$. We say that $g_{i} \cdots \cdots g_{k}$ is obtained from $h_{i} \cdots \cdots h_{k}$ by a Hurwitz move if $\exists 1 \leq p \leq k-1$ s.t. $g_{i}=h_{i} i \neq p, p+1, g_{p}=h_{p} h_{p+1} h_{p}^{-1}$ and $g_{p+1}=h_{p}$ or $g_{p}=h_{p+1}$ and $g_{p+1}=h_{p+1}^{-1} h_{p} h_{p+1}$

Definition. Hurwitz equivalence of factorized expressions

Let $g_{i} \cdots g_{k}=h_{i} \cdots h_{k}$ be two factorized expressions of the same element in a group $G$. We say that $g_{i} \cdots \cdots g_{k}$ is a Hurwitz equivalent to $h_{i} \cdots h_{k}$ if $h_{i} \cdots g_{k}$ is obtained from $h_{i} \cdots h_{k}$ by a finite number of Hurwitz moves. We denote it by $g_{1} \cdots g_{k} \underset{\overline{H e}}{\widetilde{C}} h_{i} \cdots h_{k}$. 
Definition. Factorized expression in $B_{m}$ invariant under $h \in B_{m}$

We say that a factorized expression $g_{1} \ldots g_{t}$ is invariant under $h$ if $\left(g_{1}\right)_{h}$. $\cdots\left(g_{t}\right)_{h}$ is Hurwitz equivalent to $g_{1} \ldots g_{t}$, i.e., can be obtained from $g_{1} \ldots g_{t}$ by a finite number of Hurwitz moves $\left((g)_{h}=h^{-1} g h\right)$.

Lemma 0.2. If a factorized expression $\varepsilon=g_{1} \ldots g_{t}$ in $B_{m}$ is invariant under $h \in B_{m}$, then $h$ induces an automorphism of $G(\varepsilon)$.

Proof. The group $B_{m}$ acts on $\pi_{1}(D-K, u)$; thus there is a natural action of $h \in B_{m}$ on $\pi_{1}(D-k, u)=\mathbb{F}_{m}$. Therefore, $h$ induces an automorphism of $\mathbb{F}_{m}$. Since $\varepsilon$ is Hurwitz equivalent to $(\varepsilon)_{h}$, we get that $h^{-1} M(\varepsilon) h=M(\varepsilon)$ and thus $(N(\varepsilon)) h \subset N(\varepsilon)$, and $h$ induces an automorphism of $\mathbb{F} / N(\varepsilon)=G(\varepsilon)$.

Certain factorized expressions of $\Delta_{m}^{2}$ in $B_{m}$ play an important role in the computation of the fundamental group of complements of curves, as we shall see in Theorem 0.3 .

Let $S$ be a curve in $\mathbb{C P}^{2}$ of degree $m$.

We refer the reader to BGT I, Chapter VI, [MoTe4] for the definition of a certain factorized expression in $B_{m}$ related to $S$ : S-factorization of $\Delta_{\mathbf{m}}^{2}$ or S-factorization or product form of $\Delta_{\mathrm{m}}^{\mathbf{2}}$ or braid monodromy factorization w.r.t. $S$ and $u$.

Theorem 0.3. Zariski-Van Kampen Theorem. (see [VK]) Let $\bar{S}$ be a curve in $\mathbb{C P}^{2}$ of $\operatorname{deg} m$, s.t. $\bar{S}$ is transversal to the line in infinity. Let $S=\bar{S} \cap \mathbb{C}^{2}$. Let $\varepsilon$ be the braid monodromy factorization w.r.t. to $S$ and $u$. Let $\mathbb{C}_{u}=u \times \mathbb{C}$. Let $\left\{\Gamma_{i}\right\}$ be a g-base of $\pi_{1}\left(\mathbb{C}_{u}-S, u\right)$. Then:

$\quad \pi_{1}\left(\mathbb{C}^{2}-S, *\right)=G(\varepsilon)$ and $\pi_{1}\left(\mathbb{C P}^{2}-\bar{S}, *\right)=G(\varepsilon)$ with extra relation
$\prod_{i=1}^{m} \Gamma_{i}=1$.

Remark. We shall use this theorem for $S_{3}$, the branch curve of a generic projection of $V_{3}$ (the Veronese of order 3) to $\mathbb{C P}^{2}$. In BGT IV we computed the braid monodromy factorization related to $S_{3}$. We denote it $\varepsilon(18)$. We shall again present $\varepsilon(18)$ in the next chapter.

We are going to reformulate the Zariski-Van Kampen Theorem in a more precise form for a cuspidal curve, i.e., for a curve with only nodes and cusps. 
Theorem 0.4. (Zariski). If $S$ is a cuspidal curve, then the related braid monodromy factorization $\varepsilon$ is of the form $\prod_{j=1}^{p} V_{j}^{\nu_{j}}$, where $V_{j}$ is a half-twist and $\nu_{j}=1$ or 2 or 3 .

Theorem 0.5. Zariski-Van Kampen (precise version). Let $\bar{S}$ be a cuspidal curve in $\mathbb{C P}^{2}$. Let $S=\mathbb{C}^{2} \cap \bar{S}$. Let $\varepsilon$ be a braid monodromy factorization w.r.t. $S$ and $u$. Let $\varepsilon=\prod_{j=1}^{p} V_{j}^{\nu_{j}}$, where $V_{j}$ is a half-twist and $\nu_{j}=1,2,3$.

For every $j=1 \ldots p$ let $A_{j}, B_{j} \in \pi_{1}\left(\mathbb{C}_{u}-S, u\right)$ be such that $A_{j}, B_{j}$ can be extended to a g-base of $\pi_{1}\left(\mathbb{C}_{u}-S, u\right)$ and $\left(A_{j}\right) V_{j}=B_{j}$. Let $\left\{\Gamma_{i}\right\}$ be a $g$-base of $\pi_{1}\left(\mathbb{C}_{u}-S, u\right)$. Then $\pi_{1}\left(\mathbb{C}^{2}-S, u\right)$ is generated by the images of $\left\{\Gamma_{i}\right\}$ in $\pi_{1}\left(\mathbb{C}^{2}-S, u\right)$ and the only relations are those implied from $\left\{V_{j}^{\nu_{j}}\right\}$, as follows:

$$
\begin{array}{lll}
A_{j} \cdot B_{j}^{-1}=1 & \text { if } & \nu_{j}=1 \\
{\left[A_{j}, B_{j}\right]=1} & \text { if } & \nu_{j}=2 \\
\left\langle A_{j}, B_{j}\right\rangle=1 & \text { if } & \nu_{j}=3
\end{array}
$$

$\pi_{1}\left(\mathbb{C P}^{2}-\bar{S}, *\right)$ is generated by $\left\{\Gamma_{i}\right\}$ with the above relations and one more relation $\prod_{i} \Gamma_{i}=1$.

Remark 0.5'. How to determine $A_{j}$ and $B_{j}$ from $V_{j}$ or how to determine $A_{V}$ and $B_{V}$ from $V=H(\sigma)$ (see formulation of Van Kampen Theorem).

To be able to use the Zariski-Van Kampen Theorem, we must know how to compute $A_{j}$ if $B_{j}$ for every $j=1 \ldots p$. Assume, for simplicity, that $u_{o}$ is below real lines and $\left\{q_{i}\right\}=\mathbb{C}_{u} \cap S$ are real points. Assume that $\rho\left(\delta_{j}\right)=V_{j}^{\nu_{j}}$, where $V_{j}=H(\sigma)$, a half-twist corresponding to a path $\sigma$ from $q_{1}$ to $q_{2}$. Take a homotopically-equivalent path $\sigma^{\prime}$ that passes through $u_{0}$. Let $\sigma_{1}, \sigma_{2}$ be the part of $\sigma^{\prime}$ from $u_{0}$ to $q_{1}, q_{2}$ respectively. Let $A_{j}=\ell\left(\sigma_{1}\right) B_{j}=\ell\left(\sigma_{2}\right)$ be the loops of $\pi_{1}\left(\mathbb{C}^{2}-S, u_{0}\right)$ built from $\sigma_{1}, \sigma_{2}$ as in the definition in the beginning of the Chapter. See Fig. 0.2 for an example how to determine $A_{V}$ and $B_{V}$ for $V=H(\sigma)$.

Proposition 0.6. If an $S$-factorization $\Delta^{2}=\prod g_{i}$ is invariant under $h \in$ $B_{m}$ then $R\left(\left(g_{i}\right)_{h}\right)$ is also a relation on $\pi_{1}\left(\mathbb{C}^{2}-S, *\right)$. 

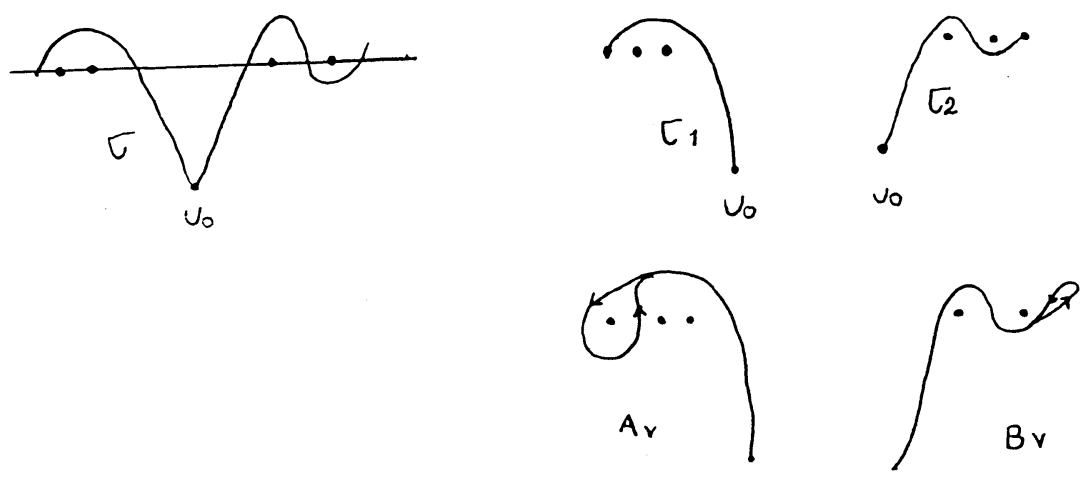

Figure 0.2 .

Proof. Zariski-Van Kampen Theorem and the fact that an invariant factorization of a braid monodromy factorization, namely $\Pi\left(g_{i}\right)_{h}$, is also a braid monodromy factorization (see Proposition VI.4.2 from BGT I, [MoTe4]).

Remark. Proposition 0.6 indicates why it is important to prove invariant properties of $S$-factorizations. We use such properties to induce more relations on the fundamental group.

I. The braid monodromy related to $\mathrm{S}$, the branch curve of a $\mathrm{V}_{3}$-projection.

Notation. $\quad V_{3}, f, S_{3}, S$

Let $V_{3}$ be the Veronese surface of order 3 , i.e., the following embedding of $\mathbb{C P}^{2}$ into $\mathbb{C P}^{N}$ :

$$
(x, y, z) \rightarrow\left(\ldots, x^{i} y^{j} z^{k}, \ldots\right)_{i+j+k=3} .
$$

Let $f=f_{3}$ be a generic projection: $V_{3} \stackrel{f}{\rightarrow} \mathbb{C P}^{2}$.

Let $S_{3}$ be its branch curve in $\mathbb{C P}^{2}$.

Let $\mathbb{C}^{2}$ be a "generic" affine piece of $\mathbb{C P}^{2}$.

Let $S=S_{3} \cap \mathbb{C}^{2}$.

$\operatorname{deg} S=\operatorname{deg} S_{3}=18$.

We are interested in $\pi_{1}\left(\mathbb{C P}^{2}-S_{3}, *\right)$ and $\pi_{1}\left(\mathbb{C}^{2}-S, *\right)$. 
We constructed in BGT III [MoTe7] a projective degeneration of $V_{3} \stackrel{f}{\rightarrow}$ $\mathbb{C P}^{2}$ into $Z^{(6)} \stackrel{f^{(6)}}{\rightarrow} \mathbb{C P}^{2}$ where $Z^{(6)}$ is a union of 9 planes $P_{j}, j=1 \ldots 9$, the ramification curve is a union of 9 intersection lines $\hat{L}_{i}, i=1 \ldots 9$, as in Fig. II.1. (Each $\hat{L}_{i}$ is an intersection line of $2 P_{j}$ 's.) $S^{(6)}$, the branch curve of $f^{(6)}$ in $\mathbb{C P}^{2}$, is a union of 9 lines $L_{i}, i=1 \ldots 9$. $\left(L_{i}=\pi^{(6)}\left(\hat{L}_{i}\right)\right)$.

$$
\begin{aligned}
& K^{(6)}=\mathbb{C} u \cap S^{(6)} . \\
& \# K^{(6)}=9 .
\end{aligned}
$$

Definition. $u, \mathbb{C}_{u}, K$.

Let us choose $u$ in the $x$-axis of $\mathbb{C}^{2}$ far away from the $x$-projection singularities of the $x$-projection of $S_{3}$ and of $S^{(6)}$.

$\mathbb{C}_{u}=u \times \mathbb{C}$.

$K=\mathbb{C}_{u} \cap S$.

From the regeneration process it is obvious that for every point $q_{i}$ that we had in $K^{(6)}$ we have 2 points $q_{t}, q_{t^{\prime}}$ in $K$ which are close to each other. Recall that we used a "real model" of $\left(\mathbb{C}_{u}, K\right)$. Thus, we assume that $K=$ $\left\{q_{i}, q_{i^{\prime}}\right\}_{i=1}^{9}, q_{i}, q_{i^{\prime}}$ are real.

Remark. For arbitrary $n$ we would get that $V_{n} \rightarrow \mathbb{C P}^{2}$ degenerated into $Z \rightarrow \mathbb{C P}^{2}$ where $Z=$ a union of $n^{2}$ planes with a ramification curve which consists of $\frac{3}{2} n(n-1)$ intersection lines, and a branch curve consisting of $\frac{3}{2} n(n-1)$ lines. Thus, $S_{n}$, the branch curve of $V_{n} \rightarrow \mathbb{C P}^{2}$, is of order $3 n(n-1)$.

In BGT IV we computed a braid monodromy factorization of $S_{3}$ denoted $\varepsilon(18)$.

We also proved those invariance properties of $\varepsilon(18)$ and invariance under complex conjugation. We shall repeat these results here. For this we have to recall some notations.

\section{Notations.}

$\underline{z}_{i j}=z_{i j}=$ a path below the real line from $q_{i}$ to $q_{j}$.

$$
Z_{i j}=H\left(z_{i j}\right)
$$

$\bar{z}_{i j} \quad=$ a path above the real line from $q_{i}$ to $q_{j}$.

$$
\bar{Z}_{i j}=H\left(\bar{z}_{i j}\right)
$$

$\underline{z}_{i j}^{(a)} \quad=$ a path above $a$ and below the real line elsewhere from $q_{i}$ to $q_{j}$. 


$$
\underline{(a)}_{i j}=H\left(\underline{z}_{i j}\right)
$$

$\bar{z}_{i j} \quad=$ a path below $a$ and $b$ above the real line elsewhere

$(a)(b)$

$$
\text { from } q_{i} \text { to } q_{j} \text {. }
$$

$$
\begin{array}{r}
\bar{Z}_{i j}=H\left(\bar{z}_{i j}\right) \\
(a)(b)
\end{array}
$$

$\rho_{i} \quad=Z_{i i^{\prime}}=H\left(z_{i i^{\prime}}\right)=$ half-twist corresponds to the shortest line between $q_{i}$ and $q_{i^{\prime}}$

$$
\begin{aligned}
Y_{i, j j^{\prime}}^{(2)} & =\prod_{m=0}^{1}\left(Y_{i j}^{2}\right) \rho_{j}^{m} \\
Y_{i i^{\prime}, j j^{\prime}}^{(2)} & =\prod_{\ell=0}^{1} \prod_{m=0}^{1}\left(Y_{i j}^{2}\right) \rho_{i}^{\ell} \rho_{j}^{m} \\
Y_{i, j j^{\prime}}^{(3)} & =\prod_{m=-1}^{1}\left(Y_{i j}^{3}\right) \rho_{j}^{m}
\end{aligned}
$$

For $\beta>\gamma$

$\underline{\tilde{z}}_{\gamma \gamma^{\prime}(\beta)}=$
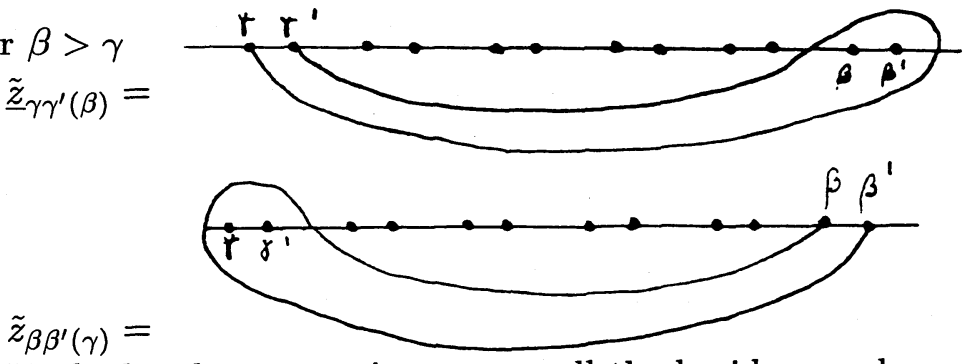

With the above notations we recall the braid monodromy factorization of $S=S^{(0)}$, denoted $\varepsilon(18)$ and some invariance properties.

Theorem I.1. $\varepsilon(18)=\prod_{\nu=7}^{1} C_{\nu} H_{\nu}$

where $H_{i}$, the braid monodromy of $S$ around $v_{i}$ factors as follows:

$$
\begin{aligned}
& H_{1}=Z_{11^{\prime}, 2}^{(3)} \cdot \tilde{Z}_{22^{\prime}(1)} \\
& H_{2}=Z_{11^{\prime}, 3}^{(3)} \cdot \tilde{Z}_{33^{\prime}(1)} \\
& H_{3}=Z_{44^{\prime}, 6}^{(3)} \cdot \tilde{Z}_{66^{\prime}(4)} \\
& H_{5}=Z_{55^{\prime}, 9}^{(3)} \cdot \tilde{Z}_{99^{\prime}(5)} \\
& H_{6}=Z_{6^{\prime}, 77^{\prime}}^{(3)} \cdot \tilde{Z}_{66^{\prime}(7)} \\
& H_{7}=Z_{8^{\prime}, 99^{\prime}}^{(3)} \cdot \tilde{Z}_{88^{\prime}(9)}
\end{aligned}
$$




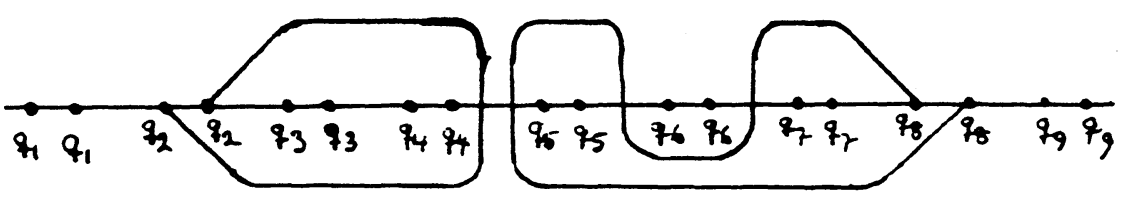

Figure I.0(a).

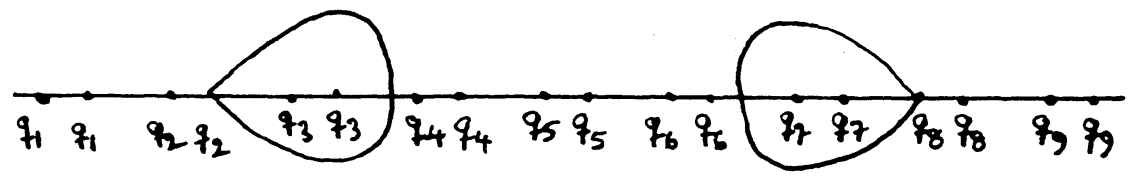

Figure I.0(b).

$$
\begin{aligned}
H_{4}= & Z_{2^{\prime}, 33^{\prime}}^{(3)} \tilde{Z}_{88^{\prime}} Z_{44^{\prime}, 8^{\prime}}^{(2)}\left(Z_{33^{\prime}, 8}^{(2)}\right)^{\bullet} \bar{Z}_{55^{\prime}, 8}^{(3)}\left(Z_{44^{\prime}, 8}^{(2)}\right)^{\bullet}\left(Z_{33^{\prime}, 8}^{(2)}\right)^{\bullet} \hat{F}_{1}\left(\hat{F}_{1}\right)_{\rho^{-1}} \\
& \cdot Z_{77^{\prime}, 8}^{(3)} Z_{2^{\prime}, i}^{2}, i=8,8,7^{\prime}, 7,5^{\prime}, 5 \quad \bar{Z}_{2^{\prime}, 44^{\prime}}^{(3)} Z_{2 i}^{2} i=8^{\prime}, 8,7^{\prime}, 7,5^{\prime}, 5 \quad \tilde{Z}_{22^{\prime}}
\end{aligned}
$$

where $\tilde{Z}_{22^{\prime}}$, $\tilde{Z}_{88^{\prime}}$ correspond to the paths $\tilde{z}_{22^{\prime}}, \tilde{z}_{88^{\prime}}$ described in Fig. I.0(a).

- denotes conjugation by a braid $b^{\bullet}$ induced from the motion described in Fig. I.0(b).

$\left(\right.$ In fact, $b^{\bullet}=Z_{2^{\prime} 3^{\prime}}^{2} Z_{2^{\prime} 3^{\prime}}^{2} Z_{7^{\prime} 8}^{-2} Z_{78}^{-2}$.)

$$
\begin{aligned}
& \hat{F}_{1}=Z_{3,44^{\prime}}^{(3)} Z_{55^{\prime}, 7}^{(3)} \alpha^{(1)}{\stackrel{(}{Z^{\prime} 7}}^{2} \bar{Z}_{3^{\prime} 7^{\prime}}^{2} \\
& \hat{F}_{2}=\left(Z_{3,44^{\prime}}^{(3)}\right) \rho^{-1}\left(Z_{55^{\prime}, 7}^{(3)}\right)_{\rho^{-1}}\left(\alpha^{(1)}\right)_{\rho^{-1}}\left(\underline{Z}_{3^{\prime} 7}^{(4)}\right)_{\rho^{-1}}\left(\bar{Z}_{3^{\prime} 7^{\prime}}\right)^{2} \rho^{-1} .
\end{aligned}
$$

$$
\alpha^{(1)}=\alpha_{1} \alpha_{2}
$$

where $\alpha_{1}$ and $\alpha_{2}$ are the curves described in Fig. I.0(c), I.0(d), respectively.

$$
\begin{aligned}
& \quad \rho=\rho_{7} \rho_{3} \\
& \quad\left(\alpha^{(1)}\right) \rho=\left(\alpha^{(1)}\right) \rho_{3}^{-1} \rho_{7}^{-1}=\left(\alpha_{1}\right)\left(\rho_{3}^{-1} \rho_{7}^{-1}\right) \cdot\left(\alpha_{2}\right)\left(\rho_{3} \rho_{7}\right)^{-1} \\
& \text { and }
\end{aligned}
$$

$$
\begin{aligned}
& C_{1}=C_{2}=I_{d} \\
& C_{3}=Z_{11^{\prime}, 44^{\prime}}^{(2)} \prod_{i=1,2,3,5} Z_{i i^{\prime}, 66^{\prime}}^{(2)}
\end{aligned}
$$

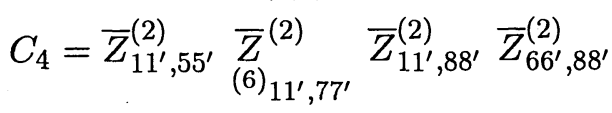




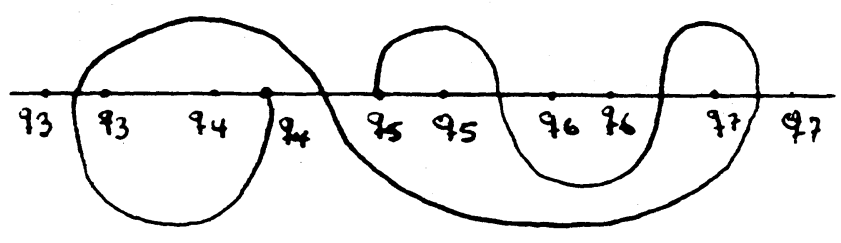

Figure I.0(c).

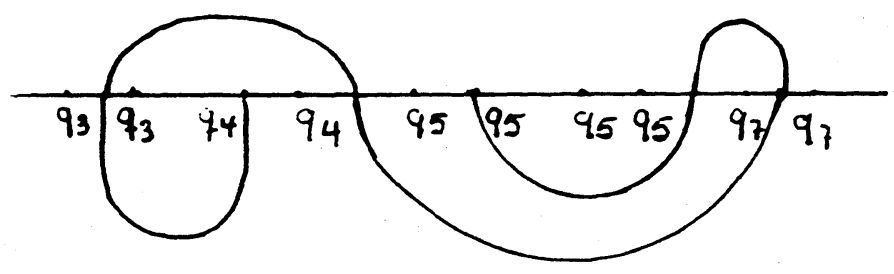

Figure I.0(d).

$$
\begin{aligned}
& C_{5}=\prod_{\substack{i=1 \\
i \neq 5}}^{7} Z_{i i^{\prime}, 99^{\prime}}^{(2)} \\
& C_{6}=C_{7}=I_{d} .
\end{aligned}
$$

The following remark gives an explicit description of a half-twist conjugated by some $\rho_{j}$ and will help us later to deduce relations from $\varepsilon(18)$ using the Van Kampen method.

\section{Remark I.1.}

(i) $Z_{i, j j^{\prime}}^{(3)}=\underline{Z}_{i j^{\prime}}^{(j)} \underline{Z}_{i j}^{3} \underline{Z}_{i j^{\prime}}^{3} \quad$ (Fig. I.1(a))

(ii) $Z_{i i^{\prime}, j j^{\prime}}^{(2)}=Z_{i j}^{2} Z_{i j^{\prime}}^{2} Z_{i^{\prime} j}^{2} Z_{i^{\prime} j^{\prime}}^{2}$ (Fig. I.1(b))

(iii) Let $Y_{i j}=H\left(y_{i j}\right)$ where $y_{i j}$ is a path connecting $q_{i}$ or $q_{i^{\prime}}$ with $q_{j}$ or $q_{j^{\prime}}$. The following graph (see Fig. I.1(c)) indicates the conjugation of $Y_{i j}$ by $\rho_{j}, \rho_{j}^{-1}, \rho_{i}, \rho_{i}^{-1}$ for different types of $y_{i j}$. In the graph we only indicate the action of $\rho_{j}$ and $\rho_{j}^{-1}$ on the "head" of $y_{i j}$ within a small circle around $q_{j}$ and $q_{j^{\prime}}$ and the action of $\rho_{i}$ and $\rho_{i}^{-1}$ on the "tail" of $y_{i j}$ in a small circle around $q_{i}$ and $q_{i^{\prime}}$. The "body" of $y_{i j}$ is not changing under $\rho_{j}^{ \pm 1}$ and $\rho_{i}^{ \pm 1}$. 
Theorem I.2. Invariance Theorem (BGT IV, Proposition 18, [MoTe7])

Let $\rho=\rho_{m_{1} \ldots m_{4}, m_{6}, m_{9}}=\rho_{1}^{m_{1}}\left(\rho_{2} \rho_{8}\right)^{m_{2}} \cdot\left(\rho_{3} \rho_{7}\right)^{m_{3}} \cdot\left(\rho_{4} \rho_{5}\right)^{m_{4}} \cdot \rho_{6}^{m_{6}} \cdot \rho_{9}^{m_{9}}$ then, $\varepsilon(18)$ is invariant under $\rho$ for every $\left.m_{i} \in \mathbb{Z} . \rho_{i}=Z_{i i^{\prime}}\right)$.

Theorem I.3. Complex Conjugation Theorem (BGT IV, Proposition 19, [MoTe7])

$\varepsilon(18)$ is invariant under complex conjugation.

A finite set of generators for $\pi_{1}\left(\mathbb{C}^{2}-\mathbf{S}, \mathbf{u}_{0}\right)$ and $\pi_{1}\left(\mathbb{C P}^{2}-\mathbf{S}_{3}, \mathbf{u}_{0}\right)$.

Let us choose $u_{0} \in \mathbb{C}_{u}, u_{0}$ below the real line. Let $\left\{\Gamma_{i}, \Gamma_{i^{\prime}}\right\}$ be a $g$ base of $\pi_{1}\left(\mathbb{C}_{u}-S, u_{o}\right)$. When considered as elements of $\pi_{1}\left(\mathbb{C}^{2}-S, u_{0}\right)$ and of $\pi_{1}\left(\mathbb{C P}^{2}-S_{3}, u_{o}\right)$, they generate (not freely) the groups. Thus we have $\left\{\Gamma_{i}, \Gamma_{i^{\prime}}\right\}_{i=1}^{9}$, a set of generators for $\pi_{1}\left(\mathbb{C}^{2}-S, u_{0}\right)$ and $\pi_{1}\left(\mathbb{C P}^{2}-S_{3}, u_{0}\right)$.

We want to compute $G=\pi_{1}\left(\mathbb{C}^{2}-S, u_{0}\right)$ and $\bar{G}=\pi_{1}\left(\mathbb{C P}^{2}-S_{3}, u_{0}\right)$.

By the Zariski-Van Kampen Theorem, $G \simeq G(\varepsilon(18))$ and $\bar{G} \cong \frac{G(\varepsilon(18))}{\prod_{i=1}^{9} \Gamma_{i} \Gamma_{i^{\prime}}}$.

Corollary I.4. $G=\pi_{1}\left(\mathbb{C}^{2}-S, u_{0}\right)$ satisfies all the relations induced in $R(\varepsilon(18))$, all the relations induced from $\left(R(\varepsilon(18)) \rho_{m_{1}, \ldots, m_{4}, m_{6}, m_{9}}\right.$ and all the relations induced from the complex conjugation of $\varepsilon(18)$, where $\rho_{m_{1} \ldots m_{4}, m_{6}, m_{9}}=\rho_{1}^{m_{1}}\left(\rho_{2} \rho_{8}\right)^{m_{2}} \cdot\left(\rho_{3} \rho_{7}\right)^{m_{3}} \cdot\left(\rho_{4} \rho_{5}\right)^{m_{4}} \cdot \rho_{6}^{m_{6}} \cdot \rho_{9}^{m_{9}}$ and $\rho_{i}=Z_{i i^{\prime}}$. Moreover,

$$
\begin{aligned}
& G \sim G(\varepsilon(18)) \\
& \bar{G}=G / \prod_{i} \Gamma_{i} \Gamma_{i^{\prime}} .
\end{aligned}
$$

Proof. Theorems I.1, I.2, I.3 and the Van Kampen Theorem.

Corollary 1.5. Let $G=\pi_{1}\left(\mathbb{C}^{2}-S, u_{0}\right)$. If $R$ is any relation in $G$ then $(R) \rho_{m_{1}, \ldots, m_{4}, m_{6}, m_{9}}$ is also a relation in $G$, where $(R) \rho_{m_{1}, \ldots, m_{4}, m_{6}, m_{9}}$ is the relation induced from $R$ by replacing $\Gamma_{i}$ and $\Gamma_{i^{\prime}}$ with $\left(\Gamma_{i}\right) \rho_{i}^{m_{i}}$ and $\left(\Gamma_{i^{\prime}}\right) \rho_{i}^{m_{i}}, i=1,2,3,4,6,9$, respectively and replacing $\Gamma_{8}, \Gamma_{8^{\prime}}, \Gamma_{7}, \Gamma_{7^{\prime}}, \Gamma_{5}, \Gamma_{5^{\prime}}$ with $\left(\Gamma_{8}\right) \rho_{8}^{m_{2}},\left(\Gamma_{8^{\prime}}\right) \rho_{8}^{m_{2}},\left(\Gamma_{7}\right) \rho_{7}^{m_{3}},\left(\Gamma_{7^{\prime}}\right) \rho_{7}^{m_{3}},\left(\Gamma_{5}\right) \rho_{5}^{m_{4}},\left(\Gamma_{5^{\prime}}\right) \rho_{5}^{m_{4}}$, respectively. 

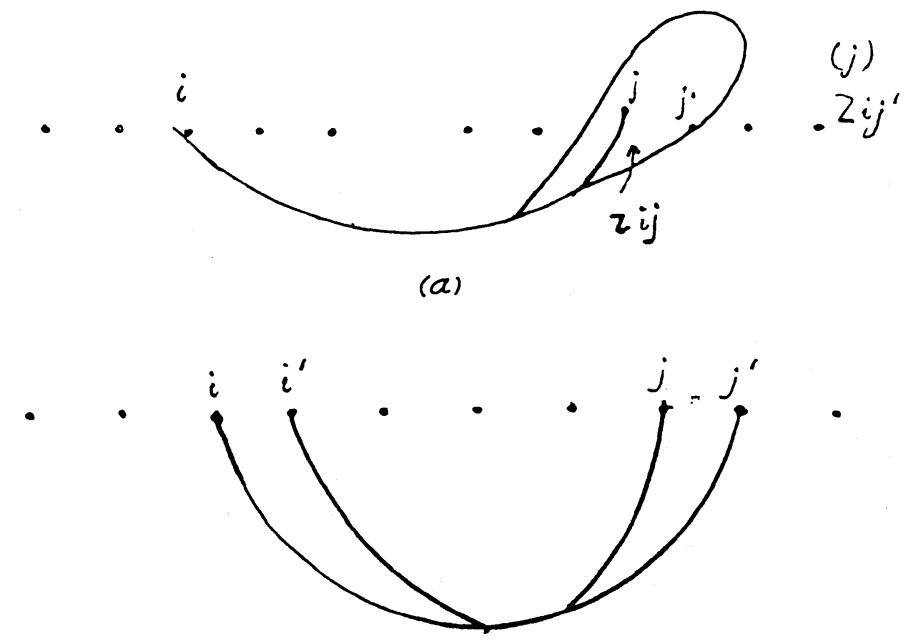

(b)
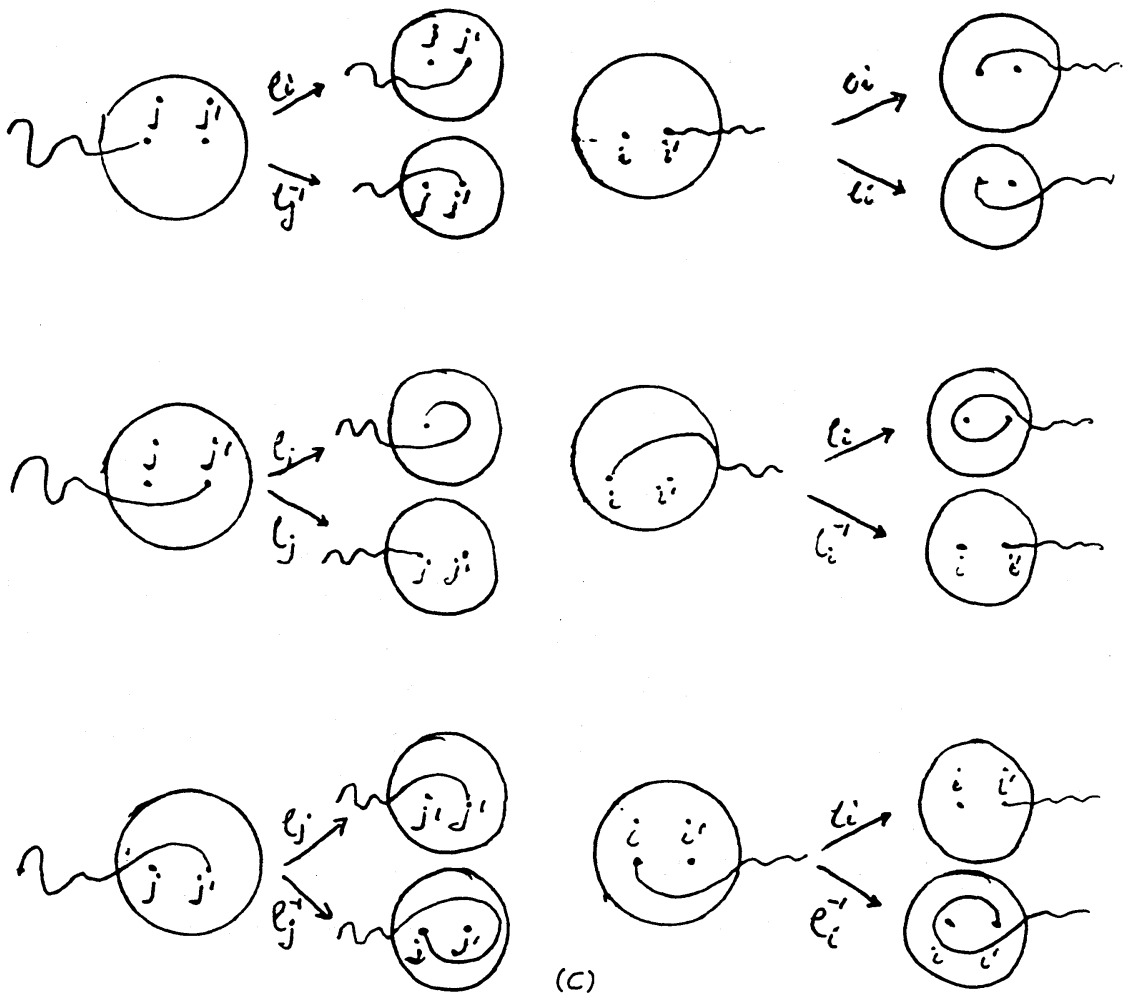

Figure I.1. 
Proof. Proposition 0.6 and Theorem I.2.

Remark. In other words, $\rho_{m_{1} \ldots m_{4}, m_{6}, m_{9}}$ defines an automorphism of $G$.

\section{Notation.}

$$
\Gamma_{\underline{i}}=\text { any element of the set }\left\{\left(\Gamma_{i}\right) \rho_{i}^{m_{i}}\right\}_{m} \in \mathbb{Z} .
$$

Corollary I.6. (Complete invariance in 3-points)

$$
\text { Let }(\alpha, \beta)=(1,2) \text { or }(1,3) \text { or }(4,6) \text { or }(5,9) \text { or }(6,7) \text { or }(8,9) \text {. Any }
$$
relation $R$ in $G$ that involves only $\Gamma_{\alpha}$ and $\Gamma_{\beta}$ is true when $\Gamma_{\underline{\alpha}}$ replaces $\Gamma_{\alpha}$ amd $\Gamma_{\underline{\beta}}$ replaces $\Gamma_{\beta}$.

Proof. Without loss of generality assume $(\alpha, \beta)=(1,2)$. Let $\Gamma_{1}=\left(\Gamma_{1}\right) \rho_{1}^{m_{1}}$ $\Gamma_{\underline{2}}=\left(\Gamma_{2}\right) \rho_{2}^{m_{2}}$. By Corollary I.5, $(R) \rho_{m_{1}, m_{2}, 0,0,0,0}$ is also a relation on $R$, and differs from $R$ by replacing $\Gamma_{1}$ by $\Gamma_{1}$ and $\Gamma_{\underline{2}}$ by $\Gamma_{2}$.

Corollary I.7. Let $R$ be a relation in $G$ that involves at most one index of each of the pairs $(2,8),(3,7),(4,5)$. For every index $i$ that appears in $R$ choose some $\Gamma_{\underline{i}}$. Then $R$ is true when we simultaneously replace $\Gamma_{i}$ by $\Gamma_{\underline{i}}$ (or $\left.E_{i}\right)$ and $\Gamma_{i}^{\prime}$ by $\left(\Gamma_{\underline{i}}\right) \rho_{i}$ (or $\left(E_{\underline{i}}\right) \rho_{i}$, respectively), for $i \in\{$ indices that appear in $R\}$.

Proof. We can assume w.l.o.g. that $\Gamma_{8}, \Gamma_{8^{\prime}}, \Gamma_{7} \Gamma_{7^{\prime}}, \Gamma_{5}, \Gamma_{5^{\prime}}$ appear in $R$ and $\Gamma_{2}, \Gamma_{2^{\prime}}, \Gamma_{3}, \Gamma_{3^{\prime}}, \Gamma_{4}, \Gamma_{4^{\prime}}$ do not appear in $R$. Let $i$ be s.t. $\Gamma_{i}$ or $\Gamma_{i^{\prime}}$ appears in $R(i \in 1,5,6,7,8,9)$. There exist $m_{i}$ s.t. $\Gamma_{\underline{i}}=\left(\Gamma_{i}\right) \rho_{i}^{m_{i}}$. Let $\rho=\left(\prod_{i=1,5,6,8,9} \rho_{i}^{m_{i}}\right) \rho_{2}^{m_{8}} \rho_{3}^{m_{7}} \rho_{4}^{m_{5}}$. By Corollary I.5 $(R) \rho$ is also a relation in $G$. Since $\Gamma_{2}, \Gamma_{2^{\prime}}, \Gamma_{3}, \Gamma_{3^{\prime}}, \Gamma_{4}, \Gamma_{4^{\prime}}$ do not appear in $R$ the relation $(R) \rho$ is actually equal to $(R) \prod_{i=1,5,6,8,9} \rho_{i}^{m_{i}}$ and it differs from $R$ by replacing $\Gamma_{i}$ with $\left(\Gamma_{i}\right) \rho_{i}^{m_{i}}$ and $\Gamma_{i^{\prime}}$ by $\left(\Gamma_{i^{\prime}}\right) \rho_{i}^{m_{i}}$ for $i=1,5,6,7,8,9$. But $\left(\Gamma_{i}\right) \rho_{i}^{m_{i}}=\Gamma_{\underline{i}}$ and $\left(\Gamma_{i^{\prime}}\right) \rho_{i}^{m_{i}}=\left(\Gamma_{i}\right) \rho_{i} \rho_{i}^{m_{i}}=\left(\Gamma_{i}\right) \rho_{i}^{m_{i}} \rho_{i}=\left(\Gamma_{\underline{i}}\right) \rho_{i}$, so we get the corollary.

Remark. We can replace every $\Gamma_{i}$ that appear in $R$ by any $\Gamma_{\underline{i}}=\left(\Gamma_{i}\right) \rho_{i}^{m_{i}}$ (i.e., different $m_{i}$ 's for different $\Gamma_{i}$ 's) since both $\Gamma_{3}$ and $\Gamma_{7}\left(\Gamma_{2}\right.$ and $\Gamma_{8}, \Gamma_{5}$ and $\Gamma_{4}$, respectively) do not appear in $R$. If both $\Gamma_{3}$ and $\Gamma_{7}$ appear in $R$, then we could only replace $\Gamma_{3}$ by $\left(\Gamma_{3}\right) \rho_{3}^{m}$ and $\Gamma_{7}$ by $\left(\Gamma_{7}\right) \rho_{7}^{m}$ for the same $m$. 


\section{List of relations in G.}

We are going to describe $G$ using a different set of generators than those introduced in Chapter I. We use all the notations from Chapter I, all the relations induced from the braid monodromy factorization $\varepsilon(18)$ (Theorem I.1), the complex conjugation (Theorem I.3), and Corollaries I.5 and I.6.

Remark II.0. First set of generators for $G$

Let $\left\{\Gamma_{i}, \Gamma_{i^{\prime}}\right\}$ be a $g$-base of $\pi_{1}\left(\mathbb{C}_{u}-S, u_{0}\right)$. Considered. as elements of $G=\pi_{1}\left(\mathbb{C}^{2}-S, u_{0}\right),\left\{\Gamma_{i}, \Gamma_{i^{\prime}}\right\}$ generates $G$.

\section{Definition.}

$$
\begin{gathered}
E_{i}= \begin{cases}\Gamma_{i} & i \neq 2,7 \\
\Gamma_{i^{\prime}} & i=2,7\end{cases} \\
E_{i^{\prime}}=\left(E_{i}\right) \rho_{i} .
\end{gathered}
$$

\section{Notations.}

$\rho_{i}=Z_{i i^{\prime}}$ the half-twist corresponding to the shortest path between $q_{j}$ and $q_{j^{\prime}}$.

$E_{\underline{i}}=$ an element of $\left\{\left(E_{i}\right) \rho_{i}^{m}\right\}_{m} \in \mathbb{Z}$.

$\Gamma_{\underline{i}}=$ an element of $\left\{\left(\Gamma_{i}\right) \rho_{i}^{m}\right\}_{m} \in \mathbb{Z}$.

In order to use the invariance theorem we need the following lemma.

Lemma II.0. (i) $\quad\left(\Gamma_{j}\right) \rho_{i}=\Gamma_{j} \quad\left(\Gamma_{j^{\prime}}\right) \rho_{i}=\Gamma_{j^{\prime}}$ for $i \neq j$.

(ii) $\quad\left(\Gamma_{i}\right) \rho_{i}=\Gamma_{i^{\prime}} \quad\left(\Gamma_{i^{\prime}}\right) \rho_{i}=\Gamma_{i^{\prime}} \Gamma_{i} \Gamma_{i^{\prime}}^{-1} \quad\left(\Gamma_{i}\right) \rho_{i}^{-1}=\Gamma_{i}^{-1} \Gamma_{i^{\prime}} \Gamma_{i}$.

(iii) Let $\rho=\rho_{m_{1}, \ldots, m_{4}, m_{6}, m_{9}}=\rho_{1}^{m_{1}}\left(\rho_{2} \rho_{8}\right)^{m_{2}} \cdot\left(\rho_{3} \rho_{7}\right)^{m_{3}} \cdot\left(\rho_{4} \rho_{5}\right)^{m_{4}} \cdot \rho_{6}^{m_{6}} \cdot \rho_{9}^{m_{9}}$. Then $\left(\Gamma_{i}\right) \rho=\Gamma_{\underline{i}}$.

(iv) $\Gamma_{\underline{i}} \in\left\langle\Gamma_{i}, \Gamma_{i^{\prime}}\right\rangle$.

Proof. Geometric observation (Fig. II.0(a) and (b)) or BGT I, Section II, $\S 2$.

Lemma II.1. (i) $E_{i^{\prime}}= \begin{cases}\Gamma_{i^{\prime}} & i \neq 2,7 \\ \Gamma_{i^{\prime}} \Gamma_{i} \Gamma_{i^{\prime}}^{-1} & i=2,7\end{cases}$ 

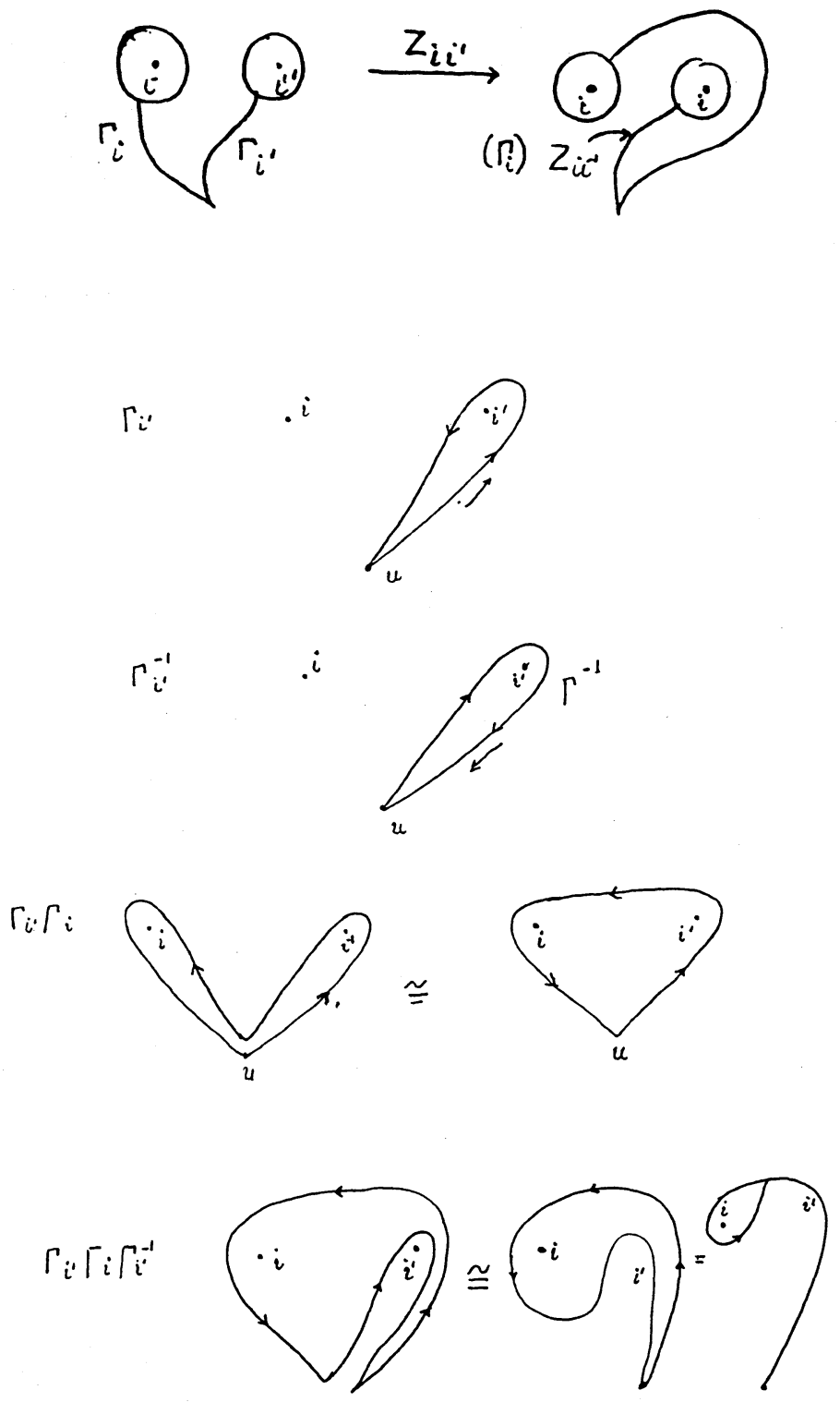

Figure II.0. 
(ii) $\Gamma_{i^{\prime}} \Gamma_{i}=E_{i^{\prime}} E_{i}$

(iii) $\left(E_{i}\right) \rho_{i}=E_{i^{\prime}}$

(iv) $\left(E_{i^{\prime}}\right) \rho_{i}=E_{i^{\prime}} E_{i} E_{i^{\prime}}^{-1}$

(v) $\Gamma_{i}= \begin{cases}E_{i} & i \neq 2,7 \\ E_{i}^{-1} E_{i^{\prime}} E_{i} & i=2,7 \quad\left(=\left(E_{i^{\prime}}\right) \rho_{i}^{-2}=\left(E_{i}\right) \rho_{i}^{-1}\right)\end{cases}$

Proof. Trivial.

Lemma II.2. $\left\{E_{i}, E_{i^{\prime}}\right\}$ generate $G$.

Proof. Trivial.

Remark II.2. A second set of generators for $G$

We start with a set of generators $\Gamma_{i}, \Gamma_{i^{\prime}}$ and exchange it for a set of generators $E_{i}, E_{i^{\prime}}$.

Definition. Let $\psi$ be the classical monodromy homomorphism from $G$ to the symmetric group of order 9 induced by the projection $V_{3} \rightarrow \mathbb{C P}^{2}$.

Lemma II.3. $\psi\left(\Gamma_{i}\right)=\psi\left(\Gamma_{i^{\prime}}\right)=\psi\left(E_{i}\right)=\psi\left(E_{i^{\prime}}\right)=\left(k_{i}, \ell_{i}\right)$ where $\hat{L}_{i}=$ $P_{k_{i}} \cap P_{\ell_{i}}$, and $\left\{L_{i}\right\}$ and $\left\{P_{j}\right\}$ are arranged as in Fig. II.1.

Proof. Let $\gamma_{i}$ be a path in $\mathbb{C}^{2}$ connecting $u_{0}$ to $q_{i}$ s.t. $\Gamma_{i}=\ell\left(\gamma_{i}\right)$ (see the definition of $\ell(\gamma)$ in Chapter 0$)$. One has to consider the degeneration of $V_{3} \stackrel{f}{\rightarrow} \mathbb{C P}^{2}$ to $Z^{(6)} \stackrel{f^{(6)}}{\rightarrow} \mathbb{C P}^{2}$ and of $S_{3}$ to $S^{(6)}$, constructed in BGT III, [MoTe7]. The surface $Z^{(6)}$ is a union of 9 planes, $P_{1} \cdots P_{9}$. The configuration of the planes and their intersection lines $\hat{L}_{1}, \ldots, \hat{L}_{9}$ are as in Fig. II.1. Let $f^{(6)}$ be a generic projection $Z^{(6)} \stackrel{f^{(6)}}{\rightarrow} \mathbb{C P}^{2}$ and $S^{(6)}$ its branch curve in $\mathbb{C}^{2}$. We choose $V_{3}$ to be close to $Z^{(6)}$. Let $p_{i}^{(6)}=P_{i} \cap \pi^{(6)^{-1}}\left(u_{0}\right)$. Let $p_{i}$ be a point in $\pi^{-1}\left(u_{o}\right)$ which is close to $p_{i}^{(6)}$. Fix $i$ between 1 and 9 . It is clear that when we move along $\gamma_{i}$ from $u_{0}$ to $q_{i}$, the lifted path in $Z^{(6)}$ which starts in $p_{k_{i}}^{(6)}$ will lie in $P_{k_{i}}$ and will end on a point in $\hat{L}_{i}$ above $q_{i}$. The lifted path in $Z^{(6)}$ 

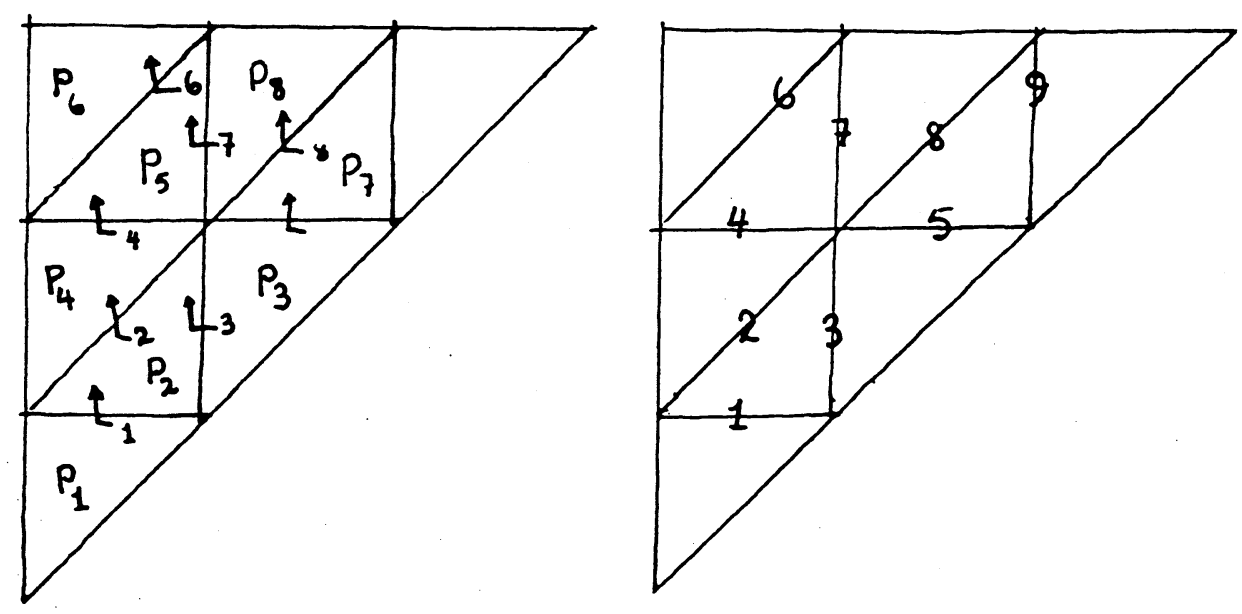

Figure II.1.

that starts in $p_{\ell_{i}}^{(6)}$ will lie in $P_{\ell_{i}}$ and will end in the same point in $\hat{L}_{i}$. Thus, in the regenerated case, the lifted path of $\Gamma_{k}$ that starts in $p_{i}$ will end in $p_{j}$. The lifted paths of $\gamma_{i}$ in $Z^{(6)}$ that start in $p_{t}^{(6)}, t \neq k_{i}, \ell_{i}$ will be closed loops. Thus, in the regenerated case, the path obtained from lifting $\Gamma_{k}$ that starts in $p_{t}, t \neq k_{i}, \ell_{i}$ is a loop. Thus, $\psi\left(\Gamma_{k}\right)=$ the transposition $\left(k_{i} \ell_{i}\right)$ of the symmetric group on 9 elements In the same way $\psi\left(\Gamma_{i^{\prime}}\right)=\left(\begin{array}{ll}k_{i} & \ell_{i}\end{array}\right)$, and thus $\psi\left(E_{i}\right)=\psi\left(E_{i^{\prime}}\right)=\left(\begin{array}{ll}k_{i} & \ell_{i}\end{array}\right)$.

Corollary II.3. The transpositions $\psi\left(E_{i}\right)$ are as follows:

$$
\begin{aligned}
& \psi\left(E_{1}\right)=\left(\begin{array}{ll}
1 & 2
\end{array}\right) \\
& \psi\left(E_{2}\right)=\left(\begin{array}{ll}
2 & 3
\end{array}\right) \\
& \psi\left(E_{3}\right)=\left(\begin{array}{ll}
2 & 4
\end{array}\right) \\
& \psi\left(E_{4}\right)=\left(\begin{array}{ll}
3 & 5
\end{array}\right) \\
& \psi\left(E_{5}\right)=\left(\begin{array}{ll}
4 & 7
\end{array}\right) \\
& \psi\left(E_{6}\right)=\left(\begin{array}{ll}
5 & 6
\end{array}\right) \\
& \psi\left(E_{7}\right)=\left(\begin{array}{ll}
5 & 8
\end{array}\right) \\
& \psi\left(E_{8}\right)=\left(\begin{array}{ll}
7 & 8
\end{array}\right) \\
& \psi\left(E_{9}\right)=\left(\begin{array}{ll}
7 & 9
\end{array}\right)
\end{aligned}
$$

Moreover, $\psi\left(E_{i}\right)$ and $\psi\left(E_{j}\right)$ have one common index $\Leftrightarrow \hat{L}_{i}$ and $\hat{L}_{j}$ are edges of some triangle in Fig. II.1. 
Proof. Immediate from the previous Lemma.

Before continuing with $G$, we want to prove some claims concerning an arbitrary group.

Claim II.4. (a) If $\langle A, B\rangle=1$, i.e., $A B A B^{-1} A^{-1} B^{-1}=1$, then

$$
\begin{aligned}
A B A & =B A B \\
A^{-1} B^{k} A & =B A^{k} B^{-1} \\
A B^{k} A^{-1} & =B^{-1} A^{k} B \\
A^{-1} B^{-1} A^{k} & =B^{k} A^{-1} B^{-1} .
\end{aligned}
$$

(b) $[A, B]=[A, C]=1 \Rightarrow[A, D]=1$ for $D \in\langle B, C\rangle=$ subgroup generated by $B, C$.

(c) $\left[A_{C}, B_{D}\right]=1,[C, B]=[C, D]=[A, D]=1 \Rightarrow[A, B]=1$.

(d) $[A, X Y Z]=[A, X][A, Y]_{X^{-1}}[A, Z]_{Y^{-1} X^{-1}}$.

(e) $[X Y, A]=[Y, A]_{X^{-1}}[X, A]$.

(f) $\left[A_{C}, B_{C}\right]=[A, B]_{C}$.

(g) $[X, Z]=1 \Rightarrow\left[X, Y_{Z}\right]=[X, Y]_{Z}$.

(h) If $\langle x, y\rangle=1$, then $\langle A x, y\rangle=1 \Leftrightarrow A_{y^{-1} x^{-1}}=A^{-1} A_{y^{-1}}$. A

Proof. (a) - (g) are easy to verify.

We shall only prove $(\mathrm{h})$ here.

If $\langle A x, y\rangle=1$, then:

$$
\begin{aligned}
& 1=\langle A x, y\rangle=A x y x y^{-1} x^{-1} A^{-1} y^{-1}
\end{aligned}
$$

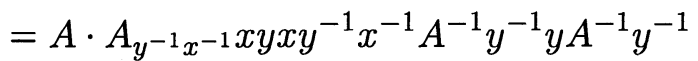

$$
\begin{aligned}
& =A \cdot A_{y^{-1} x^{-1}} \cdot 1 \cdot A_{y^{-1}}^{-1} \\
& \Rightarrow A_{y^{-1} x^{-1}} \quad=A^{-1} A_{y^{-1}} .
\end{aligned}
$$

If $A^{-1} A_{y^{-1}}=A_{y^{-1} x^{-1}}$, then

$$
\begin{aligned}
& A A_{y^{-1} x^{-1} A_{y^{-1}}^{-1}=1} \\
\Rightarrow & A x y \underbrace{A y^{-1} x^{-1} y} A^{-1} y^{-1}=1 \\
\Rightarrow & A x y \overbrace{A x y^{-1} x^{-1}} A^{-1} y^{-1}=1 \\
\Rightarrow & A x \cdot y \cdot A x \cdot y^{-1} \cdot(A x)^{-1} \cdot y^{-1}=1 \\
\Rightarrow & \langle A x, y\rangle=1 .
\end{aligned}
$$


Lemma II.5. (a) $\left[\Gamma_{i}, \Gamma_{j}\right]=\left[\Gamma_{i^{\prime}}, \Gamma_{j}\right]=1 \Rightarrow\left[\Gamma_{\underline{i}}, \Gamma_{\underline{j}}\right]=1$.

(b) $\left[\Gamma_{i}, \Gamma_{j}\right]=\left[\Gamma_{i}^{-1} \Gamma_{i^{\prime}} \Gamma_{i}, \Gamma_{j}\right]=1 \Rightarrow\left[\Gamma_{\underline{i}}, \Gamma_{j}\right]=1$.

Proof. We only prove (a); (b) is the same argument. Since $\Gamma_{j}$ is a product of $\Gamma_{j}$ and $\Gamma_{j^{\prime}}$, we can apply Claim II.4 (b) to get $\left[\Gamma_{i}, \Gamma_{j}\right]=1$. In particular, $\left[\Gamma_{i},\left(\Gamma_{j}\right) \rho_{j}^{-1}\right]=1$. We use invariance under $\rho_{i} \rho_{j}$ and Lemma 0.2 on it to get $\left[\Gamma_{i^{\prime}}, \Gamma_{j}\right]=1$. We use invariance under $\rho_{i} \rho_{j}$ and Lemma 0.2 on $\left[\Gamma_{i}, \Gamma_{j}\right]=1$ to get $\left[\Gamma_{i^{\prime}}, \Gamma_{j^{\prime}}\right]=1$. From $\left[\Gamma_{i^{\prime}}, \Gamma_{j^{\prime}}\right]=\left[\Gamma_{i^{\prime}}, \Gamma_{j}\right]=1$ we get, using Lemma II.4(b), that $\left[\Gamma_{i^{\prime}}, \Gamma_{j}\right]=1$. From $\left[\Gamma_{i}, \Gamma_{j}\right]=\left[\Gamma_{i^{\prime}}, \Gamma_{j}\right]=1$ we get, using Lemma II.4(b), that $\left[\Gamma_{\underline{i}}, \Gamma_{\underline{j}}\right]=1$.

Proposition II.6. The following relations hold in $G$ :

(1) $\left\langle E_{\underline{i}}, E_{\underline{j}}\right\rangle=1 \forall i, j$ s.t. $\psi\left(E_{i}\right)$ and $\psi\left(E_{j}\right)$ have exactly one common index.

(2) $\left[E_{\underline{i}}, E_{\underline{j}}\right]=1 \forall i, j$ s.t. $\psi\left(E_{i}\right)$ and $\psi\left(E_{j}\right)$ have no common index.

(3) $1=\left(E_{7} E_{5} E_{3}^{-1} E_{4}^{-1} E_{2} E_{4} E_{3} E_{5}^{-1} E_{7}^{-1} E_{8}^{-1}\right)\left(\rho_{3} \rho_{7}\right)^{i}\left(\rho_{4} \rho_{5}\right)^{j} \quad \forall i, j \in \mathbb{Z}$.

(4) $E_{4^{\prime}} E_{4} E_{3^{\prime}} E_{3} E_{2^{\prime}} E_{3}^{-1} E_{3^{\prime}}^{-1} E_{4}^{-1} E_{4^{\prime}}^{-1}=E_{2}$.

(5) $E_{5}^{-1} E_{5^{\prime}}^{-1} E_{7}^{-1} E_{7^{\prime}}^{-1} E_{8} E_{7^{\prime}} E_{7} E_{5^{\prime}} E_{5}=E_{8^{\prime}}$.

(6)

$$
\begin{aligned}
E_{\beta^{\prime}}=E_{\alpha}^{-1} E_{\alpha^{\prime}}^{-1} E_{\beta} E_{\alpha^{\prime}} E_{\alpha} \quad(\alpha, \beta) & =(1,2) \\
& =(1,3) \\
& =(4,6) \\
& =(5,9)
\end{aligned}
$$

(7)

$$
\begin{aligned}
E_{\alpha}=E_{\beta^{\prime}} E_{\beta} E_{\alpha^{\prime}} E_{\beta}^{-1} E_{\beta^{\prime}}^{-1} \quad(\alpha, \beta) & =(6,7) \\
& =(8,9)
\end{aligned}
$$

Definitions of $\psi\left(E_{i}\right)$ as in Corollary II.3. 
Proof. We divide the proof into the following 48 claims:

Claim 0. $\left[\Gamma_{\underline{i}}, \Gamma_{\underline{j}}\right]=1$ for $i, j$ s.t. $\hat{L}_{i} \cap \hat{L}_{j}$ do not intersect, $j \neq 9$, i.e., for $(i, j)=(1,4),(1,5),(1,6),(1,7),(1,8),(2.6),(3.6),(6.8)$.

Claim 1. $\left\langle\Gamma_{\underline{i}}, \Gamma_{\underline{j}}\right\rangle=1(i, j)=(1,2),(1,3),(4,6),(5,9),(6,7),(8,9)$

Claim 2. $\left\langle\Gamma_{2^{\prime}}, \Gamma_{\underline{3}}\right\rangle=1$.

Claim 3. $\Gamma_{3^{\prime}}^{\bullet}=\Gamma_{3} \Gamma_{2^{\prime}} \Gamma_{3}^{-1}=\Gamma_{2^{\prime}}^{-1} \Gamma_{3} \Gamma_{2^{\prime}}$.

Claim 4. $\Gamma_{3}^{\bullet}=\Gamma_{2^{\prime}}^{-1} \Gamma_{3^{\prime}}^{-1} \Gamma_{3^{\prime}} \Gamma_{3} \Gamma_{2^{\prime}}$.

Claim 5. $\left\langle\Gamma_{\underline{7}}, \Gamma_{8}\right\rangle=1$.

Claim 6. $\Gamma_{7^{\prime}}^{\bullet}=\Gamma_{8} \Gamma_{7^{\prime}} \Gamma_{7} \Gamma_{7^{\prime}}^{-1} \Gamma_{8^{\prime}}^{-1}$.

Claim 7. $\Gamma_{7}^{\bullet}=\Gamma_{8} \Gamma_{7^{\prime}} \Gamma_{8^{\prime}}^{-1}=\Gamma_{7^{\prime}}^{-1} \Gamma_{8} \Gamma_{7^{\prime}}$.

Claim 8. $\Gamma_{2^{\prime}}^{\bullet}=\Gamma_{3^{\prime}} \Gamma_{3} \Gamma_{2^{\prime}} \Gamma_{3}^{-1} \Gamma_{3^{\prime}}^{-1}$

$$
\Gamma_{8}^{\bullet}=\Gamma_{8}^{-1} \Gamma_{7^{\prime}}^{-1} \Gamma_{8} \Gamma_{7^{\prime}} \Gamma_{7} \text {. }
$$

Claim 9. $\Gamma_{2}^{\bullet}=\Gamma_{2}, \Gamma_{8^{\prime}}^{\bullet}=\Gamma_{8^{\prime}}, \Gamma_{4}^{\bullet}=\Gamma_{4}, \Gamma_{4^{\prime}}^{\bullet}=\Gamma_{4^{\prime}}, \Gamma_{5^{\prime}}^{\bullet}=\Gamma_{5^{\prime}}, \Gamma_{5}^{\bullet}=\Gamma_{5}$.

Claim 10. $\left[\Gamma_{\underline{2}}, \Gamma_{\underline{i}}\right]=1 \quad i=5,7,8$.

Claim 11. $\left[\Gamma_{8^{\prime}}, \Gamma_{i}\right]=1 \quad i=3,4$.

Claim 12. $\left[\Gamma_{\underline{8}}, \Gamma_{\underline{i}}\right]=1 \quad i=3,4$.

Claim 13. $E_{\beta^{\prime}}=E_{\alpha}^{-1} E_{\alpha^{\prime}}^{-1} E_{\beta} E_{\alpha^{\prime}} E_{\alpha} \quad(\alpha, \beta)=(1,2)$

$$
\begin{aligned}
& =(1,3) \\
& =(4,6) \\
& =(5,9) \\
E_{\alpha}=E_{\beta^{\prime}} E_{\beta} E_{\alpha^{\prime}} E_{\beta}^{-1} E_{\beta^{\prime}}^{-1}(\alpha, \beta) & =(6,7) \\
& =(8,9)
\end{aligned}
$$


Claim 14. $\left[\Gamma_{3^{\prime}}^{\bullet}, \Gamma_{7^{\prime}}^{\bullet}\right]=1$.

Claim 15. $\left[\Gamma_{3}, \Gamma_{7^{\prime}} \Gamma_{7} \Gamma_{7^{\prime}}^{-1}\right]=1$.

Claim 16. $\left[\Gamma_{3^{\prime}}, \Gamma_{7^{\prime}} \Gamma_{7} \Gamma_{7^{\prime}}^{-1}\right]=1$.

Claim 17. $\left[\Gamma_{\underline{3}}, \Gamma_{7^{\prime}} \Gamma_{7} \Gamma_{7^{\prime}}^{-1}\right]=1$.

Claim 18. $\left[\Gamma_{\underline{3}}, \Gamma_{\underline{7}}\right]=1$.

Claim 19. $\left[\Gamma_{\underline{3}}^{\bullet}, \Gamma_{\underline{7}}^{\bullet}\right]=1$.

Claim 20. $\left\langle\Gamma_{\underline{3}}^{\bullet}, \Gamma_{\underline{4}}^{\bullet}\right\rangle=1$.

Claim 21. $\left\langle\Gamma_{\underline{5}}^{\bullet}, \Gamma_{\underline{7}}^{*}\right\rangle=1$.

Claim 22. $\Gamma_{7}^{\bullet} \Gamma_{5}^{\bullet} \Gamma_{7}^{\bullet-1}=\Gamma_{3^{\prime}}^{\bullet-1} \Gamma_{4}^{\bullet} \Gamma_{3^{\prime}}^{\bullet}\left(\rho_{3} \rho_{7}\right)^{i}\left(\rho_{4} \rho_{5}\right)^{j}$.

Claim 23. $\left[\Gamma_{7}^{\bullet}, \Gamma_{4^{\prime}}^{\bullet} \Gamma_{4}^{\bullet} \Gamma_{3^{\prime}}^{\bullet} \Gamma_{4}^{\bullet-1} \Gamma_{4^{\prime}}^{\bullet-1}\right]=1$.

Claim 24. $\left\langle\Gamma_{\underline{3}}^{\circ}, \Gamma_{\underline{5}}^{\circ}\right\rangle=1$.

Claim 25. $\left\langle\Gamma_{\underline{\underline{4}}}^{\bullet}, \Gamma_{\underline{\underline{ }}}^{\bullet}\right\rangle=1$.

Claim 26. $\left\langle\Gamma_{\underline{3}}, \Gamma_{\underline{5}}\right\rangle=1$.

Claim 27. $\left\langle\Gamma_{\underline{\underline{Z}}}, \Gamma_{\underline{4}}\right\rangle=1$.

Claim 28. $\left[\Gamma_{4^{\prime}}, \Gamma_{5}\right]=1$.

Claim 29. $\left[\Gamma_{4}, \Gamma_{5}\right]=1$

Claim 30. $\left[\Gamma_{\underline{4}}, \Gamma_{5}\right]=1$.

Claim 31. $\left[\Gamma_{\underline{4}}, \Gamma_{5}\right]=1$.

Claim 32. $\left[\Gamma_{2^{\prime}}, \Gamma_{7^{\prime}}^{\bullet}\right]=1$.

Claim 33. $\left[\Gamma_{3}, \Gamma_{7}^{\bullet}\right]=1$. 
Claim 34. $\left[\Gamma_{7}^{\bullet}, \Gamma_{5}^{2} \Gamma_{3} \Gamma_{5}^{-2}\right]=1$.

Claim 35. $\left[\Gamma_{2^{\prime}}, \Gamma_{3}^{2} \Gamma_{5} \Gamma_{3}^{-2}\right]=1$.

Claim 36. $\left[\Gamma_{\underline{3}}, \Gamma_{4}\right]=1$.

Claim 37. $\left[\Gamma_{7^{\prime}}^{\bullet}, \Gamma_{4}^{2} \Gamma_{3^{\prime}}^{\bullet} \Gamma_{4}^{-2}\right]=1$.

Claim 38. $\left[\Gamma_{7^{\prime}}, \Gamma_{3^{\prime}}^{\bullet}\right]=1, \quad\left[\Gamma_{8}, \Gamma_{3^{\prime}}^{\bullet}\right]=1$.

Claim 39. $\left[\Gamma_{4}, \Gamma_{7^{\prime}}^{2} \Gamma_{8} \Gamma_{7^{\prime}}^{-2}\right]=1$.

Claim 40. $\left[\Gamma_{5}, \Gamma_{7^{\prime}}\right]=1$.

Claim 41. $\left[\Gamma_{\underline{5}}, \Gamma_{\underline{7}}\right]=1$.

Claim 42. $\left\langle\Gamma_{\underline{4}}, \Gamma_{\underline{2}}\right\rangle=1$.

Claim 43. $\left\langle\Gamma_{\underline{2}}, \Gamma_{\underline{3}}\right\rangle=1$. $\left\langle\Gamma_{\underline{7}}, \Gamma_{\underline{8}}\right\rangle=1$.

Claim 44. $\left\langle\Gamma_{\underline{5}}, \Gamma_{\underline{8}}\right\rangle=1$.

Claim 45. $\Gamma_{8}=\Gamma_{7^{\prime}} \Gamma_{5} \Gamma_{3}^{-1} \Gamma_{4}^{-1} \Gamma_{2^{\prime}} \Gamma_{4} \Gamma_{3} \Gamma_{5}^{-1} \Gamma_{7^{\prime}}^{-1}$.

Claim 46. $\Gamma_{2^{\prime}}=\Gamma_{4^{\prime}} \Gamma_{4} \Gamma_{3^{\prime}} \Gamma_{3} \Gamma_{2^{\prime}} \Gamma_{2} \Gamma_{2^{\prime}}^{-1} \Gamma_{4}^{-1} \Gamma_{3}^{-1} \Gamma_{3^{\prime}}^{-1} \Gamma_{4}^{-1} \Gamma_{4^{\prime}}^{-1}$.

Claim 47. $\Gamma_{8^{\prime}}=\Gamma_{5}^{-1} \Gamma_{5^{\prime}}^{-1} \Gamma_{7}^{-1} \Gamma_{7^{\prime}}^{-1} \Gamma_{8} \Gamma_{7^{\prime}} \Gamma_{7} \Gamma_{5^{\prime}} \Gamma_{5}$.

Claim 48. $\left[\Gamma_{\underline{i}}, \Gamma_{\underline{9}}\right]=1 \quad i=1,2,3,4,6,7$.

Proofs of the Claims.

We use the braids in $\varepsilon(18)$ (see Theorem I.1) to induce relations on $G$ via the Van Kampen Theorem (Theorem 0.5). For every factor $V^{\nu}$ in $\varepsilon(18)$, we have to find $A_{V}$ and $B_{V}$ to get a relation. In Remark 0.4 one can find an algorithm how to determine $A_{V}$ and $B_{V}$. In $\varepsilon(18)$ we have sometimes used a compact notation for a product of a few factors. Then we use Remark I.1 to determine the factors precisely. Sometimes, instead of using a factor $b$ in $\varepsilon(18)$, we shall use its complex conjugation $\bar{b}$. In that way, we get $R(b)$ 
and/or $R(\bar{b})$ a relation on $G$ induced by the Van Kampen Theorem. We also use Corollary I.5 to get other relations using $(R) \rho_{m_{1}, \ldots, m_{4}, m_{6}, m_{9}}$.

Proof of Claim 0. Taking the factors $C_{i}^{\prime}$ or the complex conjugate of $C_{i}^{\prime} i=$ $1 \ldots 4$ and applying the Van Kampen method on it to produce relations on $G$, we get $\left[\Gamma_{i i^{\prime}}, \Gamma_{j j^{\prime}}\right]=1 \forall i, j$ s.t. $\hat{L}_{i} \cap \hat{L}_{j}=\emptyset j \neq 7,9$. By Lemma II.5 we get $\left[\Gamma_{\underline{i}}, \Gamma_{\underline{j}}\right]=1 \forall i, j$ s.t. $\hat{L}_{i} \cap \hat{L}_{j}=\emptyset j \neq 7,9$. For $j=7$ we consider $C_{4}^{\prime}$. In $C_{4}^{\prime}$ we have $\tilde{Z}_{17}=\underset{\left(6,6^{\prime}\right)}{\bar{Z}} \quad$ :

Its complex conjugate is $\stackrel{(6,6}{\underline{Z}}_{17}$ :

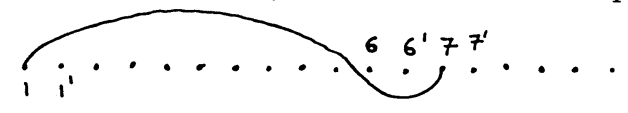
which implies on $G$ the relation:

$$
\left[\Gamma_{1}, \Gamma_{6}^{-1} \Gamma_{6^{\prime}}^{-1} \Gamma_{7} \Gamma_{6^{\prime}} \Gamma_{6}\right]=1 .
$$

Since we already know that $\left[\Gamma_{1}, \Gamma_{6}\right]=1$ we get $\left[\Gamma_{1}, \Gamma_{7}\right]=1$. Now, we are using the Invariance Theorem (Corollary I.6) and we get $\left[\Gamma_{\underline{1}}, \Gamma_{\underline{7}}\right]=1$.

Proof of Claim 1. From $H_{i}^{\prime}, i \neq 4$ and Corollary I.5.

Proof of Claim 2. By using $Z_{2^{\prime}, 33^{\prime}}^{(3)}$ of $H_{4}^{\prime}$ we get $\left\langle\Gamma_{2^{\prime}}, \Gamma_{3}\right\rangle=\left\langle\Gamma_{2^{\prime}}, \Gamma_{3^{\prime}}\right\rangle=$ $\left\langle\Gamma_{2^{\prime}}, \Gamma_{3^{\prime}} \Gamma_{3} \Gamma_{3^{\prime}}^{-1}\right\rangle$. We apply on it the invariance automorphism $\left(\rho_{3} \rho_{7}\right)^{m_{3}}$ for all possible values of $m_{3}$ to get $\left\langle\Gamma_{2^{\prime}}, \Gamma_{3}\right\rangle=1$.

Proof of Claim 3. By definition of $\bullet$ (see Theorem I.1).

$$
\begin{aligned}
\Gamma_{3^{\prime}}^{\bullet} & =\Gamma_{3^{\prime}} \Gamma_{3} \Gamma_{2^{\prime}} \Gamma_{3}^{-1} \Gamma_{3^{\prime}} \Gamma_{3} \Gamma_{2^{\prime}}^{-1} \Gamma_{3}^{-1} \Gamma_{3}^{-1} \\
& =\Gamma_{3^{\prime}} \Gamma_{3}\left(\Gamma_{3}^{-1} \Gamma_{3^{\prime}} \Gamma_{3}\right)^{-1} \Gamma_{2^{\prime}}\left(\Gamma_{3}^{-1} \Gamma_{3^{\prime}} \Gamma_{3}\right) \cdot \Gamma_{3}^{-1} \Gamma_{3}^{-1} \\
& \text { by Claims } 2 \text { and II.4(a) } \Gamma_{3} \Gamma_{2^{\prime}} \Gamma_{3}^{-1} \\
& \stackrel{=}{=} \stackrel{2}{=} \stackrel{\text { and II.4(a) }}{=} \Gamma_{2^{\prime}}^{-1} \Gamma_{3} \Gamma_{2^{\prime}} .
\end{aligned}
$$

Proof of Claim 4. By definition of $\bullet$ (see Theorem I.1).

$$
\Gamma_{3^{\prime}}^{\bullet}=\Gamma_{3^{\prime}} \Gamma_{3} \Gamma_{2^{\prime}} \underbrace{\Gamma_{3}^{-1} \Gamma_{3^{\prime}}^{-1} \Gamma_{3} \Gamma_{3^{\prime}} \Gamma_{3}} \Gamma_{2^{\prime}}^{-1} \Gamma_{3}^{-1} \Gamma_{3}^{-1}
$$




$$
\begin{aligned}
& \text { by Claims } 2 \text { and II.4(a) } \Gamma_{3^{\prime}} \Gamma_{3}\left(\Gamma_{3}^{-1} \Gamma_{3^{\prime}}^{-1} \Gamma_{3} \Gamma_{3^{\prime}} \Gamma_{3}\right)^{-1} \Gamma_{2^{\prime}} \\
& \qquad\left(\Gamma_{3}^{-1} \Gamma_{3^{\prime}}^{-1} \Gamma_{3} \Gamma_{3} \Gamma_{3^{\prime}} \Gamma_{3}\right) \Gamma_{3}^{-1} \Gamma_{3^{\prime}}^{-1} \\
& =\Gamma_{3}^{-1} \Gamma_{3^{\prime}} \Gamma_{3} \Gamma_{2^{\prime}} \Gamma_{3}^{-1} \Gamma_{3^{\prime}}^{-1} \Gamma_{3} \\
& \text { by Claims } 2 \text { and II.4(a) } \Gamma_{2^{\prime}}^{-1} \Gamma_{3}^{-1} \Gamma_{3^{\prime}} \Gamma_{3} \Gamma_{2^{\prime}} .
\end{aligned}
$$

Proof of Claims 5,6,7. Arguments symmetric to 2, 3, 4 .

Proof of Claim 8. Easy to see from the geometric observation of the action of $b^{\bullet}$.

Proof of Claim 9. $b^{\bullet}$ does not affect those loops.

Proof of Claim 10. By $H_{4}^{\prime}$ we have $Z_{2^{\prime}, i}^{2}$ and $Z_{2 i}^{2}$ for $i=8,8^{\prime}, 7,7^{\prime}, 5,5^{\prime}$. Thus, by the Van Kampen Theorem, $\left[\Gamma_{2^{\prime}}, \Gamma_{i i^{\prime}}\right]=\left[\Gamma_{2}, \Gamma_{i i^{\prime}}\right]=1 \quad i=5,7,8$. By Claim II.5, we have $\left[\Gamma_{2^{\prime}}, \Gamma_{\underline{i}}\right]=\left[\Gamma_{2}, \Gamma_{\underline{i}}\right]=1 \quad i=5,7,8$. By Claim II.5, again $\left[\Gamma_{\underline{2}}, \Gamma_{\underline{i}}\right]=1$.

Proof of Claim 11. By $H_{4}^{\prime}$ we have $Z_{44^{\prime}, 8^{\prime}}^{(2)}$. Using the Van Kampen method $\left[\Gamma_{4}, \Gamma_{8^{\prime}}\right]=1$ and $\left[\Gamma_{4^{\prime}}, \Gamma_{8^{\prime}}\right]=1$ By Lemma II.5, $\left[\Gamma_{4}, \Gamma_{8^{\prime}}\right]=1$. We have in $H_{4}^{\prime}$, $\left(Z_{33^{\prime}, 8^{\prime}}\right)^{2 \bullet}$. By the Van Kampen method we have $\left[\Gamma_{3}^{\bullet}, \Gamma_{8^{\prime}}^{\bullet}\right]=\left[\Gamma_{3^{\prime}}^{\bullet}, \Gamma_{8^{\prime}}^{\bullet}\right]=1$. By Claims 4, 3, and 9, $\Gamma_{3^{\prime}}^{\cdot}=\Gamma_{2^{\prime}}^{-1} \Gamma_{3} \Gamma_{2^{\prime}}, \Gamma_{3}=\Gamma_{2^{\prime}}^{-1} \Gamma_{3}^{-1} \Gamma_{3^{\prime}} \Gamma_{3} \Gamma_{2^{\prime}}, \quad \Gamma_{8^{\prime}}^{\circ}=$ $\Gamma_{8^{\prime}}$ and thus $\left[\Gamma_{2^{\prime}}^{-1} \Gamma_{3} \Gamma_{2^{\prime}}, \Gamma_{8^{\prime}}\right]=\left[\Gamma_{2^{\prime}}^{-1} \Gamma_{3}^{-1} \Gamma_{3^{\prime}} \Gamma_{3} \Gamma_{2^{\prime}}, \Gamma_{8^{\prime}}\right]=1 .\left[\Gamma_{3^{\prime}} ; \Gamma_{8^{\prime}}\right]=1$. Since $\left[\Gamma_{2^{\prime}}, \Gamma_{8}\right]=1$, (Claim 10) we get by Lemma II.4(g), $\left[\Gamma_{3^{\prime}}, \Gamma_{8^{\prime}}\right]=1$ and $\left.\Gamma_{3}^{-1} \Gamma_{3^{\prime}} \Gamma_{3}, \Gamma_{8^{\prime}}\right]=1$. By Lemma II.5, $\left[\Gamma_{\underline{3}}, \Gamma_{8^{\prime}}\right]=1$.

Proof of Claim 12. By Claim 11, $\left[\Gamma_{\underline{3}}, \Gamma_{8^{\prime}}\right]=1$. We apply on it Corollary I.5 with $\left(Z_{88^{\prime}} Z_{22^{\prime}}\right)^{m}$ to get $\left[\Gamma_{\underline{3}}, \Gamma_{\underline{8}}\right]=1$. Similarly, we get $\left[\Gamma_{\underline{4}}, \Gamma_{\underline{8}}\right]=1$. 
Proof of Claim 13. We shall only prove $(\alpha, \beta)=(1,2)$. In $H_{1}^{\prime}$ we have $\tilde{Z}_{22^{\prime}(1)}$. By the Van Kampen Theorem we get $\Gamma_{2}=\Gamma_{1}^{-1} \Gamma_{1^{\prime}}^{-1} \Gamma_{2}^{-1} \Gamma_{2^{\prime}} \Gamma_{2} \Gamma_{1^{\prime}} \Gamma_{1}$. We apply on it $Z_{22^{\prime}}^{2}$ to get

$$
\Gamma_{2^{\prime}} \Gamma_{2} \Gamma_{2^{\prime}}^{-1}=\Gamma_{1}^{-1} \Gamma_{1^{\prime}}^{-1} \Gamma_{2^{\prime}} \Gamma_{1^{\prime}} \Gamma_{1}
$$

Thus, $E_{2}^{\prime}=E_{1}^{-1} E_{1^{\prime}}^{-1} E_{2^{\prime}} E_{1^{\prime}} E_{1}$. The other relations are induced from $H_{2}^{\prime}, H_{3}^{\prime}, H_{5}^{\prime}, H_{6}^{\prime}, H_{7}^{\prime}$.

Proof of Claim 14. In $\hat{F}$ we have $\left(\bar{Z}_{3^{\prime} 7^{\prime}}^{2}\right)^{\bullet}$. After complex conjugation this braid transforms to $\left(\underline{Z}_{3^{\prime} 7^{\prime}}^{2}\right)^{\bullet}$. By Corollary I.4, $\left(\underline{Z}_{3^{\prime} 7^{\prime}}^{2}\right)^{\bullet}$ implies, via the Van Kampen method, the following relation on $G$ : $\left[\Gamma_{3^{\prime}}^{\bullet}, \Gamma_{7^{\prime}}^{\bullet}\right]=1$.

Proof of Claim 15.

$$
\begin{aligned}
1 \stackrel{\text { Claim } 14}{=}\left[\Gamma_{3^{\prime}}^{\bullet}, \Gamma_{7^{\prime}}^{\bullet}\right] & =\left[\Gamma_{2^{\prime}}^{-1} \Gamma_{3} \Gamma_{2^{\prime}}, \Gamma_{8} \Gamma_{7^{\prime}} \Gamma_{7} \Gamma_{7^{\prime}}^{-1} \Gamma_{8}\right] \\
& \text { Claims } \stackrel{10 \text { and II.4(g) }}{=}\left[\Gamma_{3}, \Gamma_{8} \Gamma_{7^{\prime}} \Gamma_{7} \Gamma_{7^{\prime}}^{-1} \Gamma_{8}\right] \\
& \stackrel{\text { Claims } 12 \text { and II.4(g) }}{=}\left[\Gamma_{3}, \Gamma_{7^{\prime}} \Gamma_{7} \Gamma_{7^{\prime}}^{-1}\right] .
\end{aligned}
$$

Proof of Claim 16.

By Claim 1: $\left[\Gamma_{1}, \Gamma_{7}\right]=1$. Thus, $\left[\Gamma_{1^{\prime}} \Gamma_{1}, \Gamma_{7^{\prime}} \Gamma_{7} \Gamma_{7^{\prime}}^{-1}\right]=1$.

By Claim 15: $\left[\Gamma_{3}, \Gamma_{7^{\prime}} \Gamma_{7} \Gamma_{7^{\prime}}^{-1}\right]=1$.

Thus, $\left[\Gamma_{1}^{-1} \Gamma_{1^{\prime}}^{-1} \Gamma_{3} \Gamma_{1^{\prime}} \Gamma_{1}, \Gamma_{7^{\prime}} \Gamma_{7} \Gamma_{7^{\prime}}^{-1}\right]=1$.

But by Claim 13: $\Gamma_{3^{\prime}}=\Gamma_{1}^{-1} \Gamma_{1^{\prime}}^{-1} \Gamma_{3} \Gamma_{1^{\prime}} \Gamma_{1}$.

Thus, $\left[\Gamma_{3^{\prime}}, \Gamma_{7^{\prime}} \Gamma_{7} \Gamma_{7^{\prime}}^{-1}\right]=1$.

Proof of Claim 1\%. Claim 16, Claim 15 and Claim II.4(b).

Proof of Claim 18. We apply $\left(Z_{33^{\prime}} Z_{77^{\prime}}\right)^{m}$ on $\left[\Gamma_{3^{\prime}}, \Gamma_{7^{\prime}} \Gamma_{7} \Gamma_{7^{\prime}}^{-1}\right]=1$ and on $\left[\Gamma_{3}, \Gamma_{7^{\prime}} \Gamma_{7} \Gamma_{7^{\prime}}^{-1}\right]=1$ to get $\left[\Gamma_{3}, \Gamma_{7^{\prime}}\right]=\left[\Gamma_{3} \Gamma_{3^{\prime}} \Gamma_{3}^{-1}, \Gamma_{7^{\prime}}\right]=1$. By Claim II.4(b) $\left.\Gamma_{\underline{3}}, \Gamma_{7^{\prime}}\right]=1$. We use Claim 17 and Claim II.4(b) to get $\left[\Gamma_{\underline{3}}, \Gamma_{\underline{7}}\right]=1$. 
Proof of Claim 19. $\left[\Gamma_{2^{\prime}}, \Gamma_{8}\right]=\left[\Gamma_{2^{\prime}} \Gamma_{7}\right]=\left[\Gamma_{2^{\prime}} \Gamma_{7^{\prime}}\right]\left(\right.$ Claim 10), thus $\left[\Gamma_{2^{\prime}}, \Gamma_{7^{\prime}}^{\bullet}\right]=$ $\left[\Gamma_{2^{\prime}}, \Gamma_{7}^{*}\right]=1 \Rightarrow\left[\Gamma_{2^{\prime}}, \Gamma_{7}^{*}\right]=1$. Now, $\left[\Gamma_{\underline{3}}, \Gamma_{7}\right]=\left[\Gamma_{\underline{3}}, \Gamma_{8}\right]=1$ (Claim 11, Claim 18). Thus, $\left[\Gamma_{\underline{3}}, \Gamma_{\underline{7}}^{\bullet}\right]=1$. From $\left[\Gamma_{2^{\prime}}, \Gamma_{\underline{7}}^{\bullet}\right]=\left[\Gamma_{\underline{3}}, \Gamma_{7}^{\circ}\right]=1$, we get $\left[\Gamma_{3}^{\circ}, \Gamma_{\underline{7}}^{\bullet}\right]=$ $\left[\Gamma_{3^{\prime}}^{\circ}, \Gamma_{\underline{7}}^{*}\right]=1$. Thus, $\left[\Gamma_{\underline{3}}^{\circ}, \Gamma_{\underline{7}}^{*}\right]=1$.

Proof of Claim 20. In $\hat{F}$ we have $\left(Z_{33^{\prime}, 44^{\prime}}^{(3)}\right)^{\bullet}$, which by the Van Kampen Theorem implies $\left\langle\Gamma_{3}^{\circ}, \Gamma_{4}^{*}\right\rangle=1$. We apply on it $\left(Z_{33^{\prime}} Z_{77^{\prime}}\right)^{m_{3}}\left(Z_{44^{\prime}} Z_{55^{\prime}}\right)^{m_{4}}$ for all possible $m_{4}$ and $m_{3}$ to get $\left\langle\Gamma_{\underline{3}}^{\bullet}, \Gamma_{\underline{4}}^{*}\right\rangle=1 \quad\left(\left\{\left(\Gamma_{3}^{*}\right) \rho^{m}\right\}=\Gamma_{\underline{3}}^{*}\right)$.

Proof of Claim 21. Same proof as Claim 20.

Proof of Claim 22. In $\hat{F}$ we have $\alpha_{2}^{\bullet}$ where $\alpha_{2}$ is described in Fig.I.0(d). By the Van Kampen Theorem we get

$$
\Gamma_{7}^{\bullet} \Gamma_{5^{\prime}}^{\bullet} \Gamma_{7}^{\bullet-1}=\Gamma_{4^{\prime}}^{\bullet} \Gamma_{4}^{\bullet} \Gamma_{3^{\prime}}^{\bullet} \Gamma_{4}^{\bullet} \Gamma_{3^{\prime}}^{\bullet^{-1}} \Gamma_{4}^{\bullet-1} \Gamma_{4^{\prime}}^{\bullet^{-1}} .
$$

Since, $\left\langle\Gamma_{\underline{4}}^{\bullet}, \Gamma_{\underline{3}}^{\bullet}\right\rangle=1$ we can apply Claim II.4(a) to get

$$
\begin{aligned}
& =\Gamma_{4^{\prime}}^{\bullet} \Gamma_{4}^{\bullet} \Gamma_{4}^{\bullet-1} \Gamma_{3^{\prime}}^{\bullet} \Gamma_{4}^{\bullet} \Gamma_{4}^{\bullet^{-1}} \Gamma_{4^{\prime}}^{\bullet-1} \\
& =\Gamma_{4^{\prime}}^{\bullet} \Gamma_{3^{\prime}}^{\bullet} \Gamma_{4^{\prime}}^{\bullet-1} .
\end{aligned}
$$

We apply Claim II.4(a) again to get

$$
=\Gamma_{3^{\prime}}^{\bullet^{-1}} \Gamma_{4^{\prime}}^{\bullet} \Gamma_{3^{\prime}}^{\bullet}
$$

Proof of Claim 23. Directly from $\left(\underline{\underline{Z}}_{3^{\prime} 7}^{\circ}\right)^{2}$ in $\hat{F}$.

Proof of Claim 24.

By Claim $20\left\langle\Gamma_{3^{\prime}}^{\bullet} \Gamma_{3}^{\bullet} \Gamma_{3^{\prime}}^{0^{-1}}, \Gamma_{4}^{\bullet}\right\rangle=1$.

$\operatorname{Thus}\left\langle\Gamma_{3}^{\bullet}, \Gamma_{3^{\prime}}^{\circ-1} \Gamma_{4}^{\bullet} \Gamma_{3^{\prime}}^{\bullet}\right\rangle=1$.

Since $\left[\Gamma_{3}^{\bullet}, \Gamma_{7}^{\bullet}\right]=1,\left\langle\Gamma_{3}^{\bullet}, \Gamma_{7}^{\circ-1} \Gamma_{3^{\prime}}^{-1} \Gamma_{4}^{\bullet} \Gamma_{3^{\prime}}^{\bullet} \Gamma_{7}^{\bullet}\right\rangle=1$. 
By Claim $22\left\langle\Gamma_{3^{\prime}}^{\bullet}, \Gamma_{5}^{\bullet}\right\rangle=1$

Proof of Claim 25. Follows from Claim 21, as in Claim 24.

Proof of Claim 26. Follows from Claim 24, using $\left[\Gamma_{2^{\prime}}, \Gamma_{5}\right]=1$.

Proof of Claim 27. From Claim 25, using $\left[\Gamma_{8}, \Gamma_{4}\right]=1$.

Proof of Claim 28.

By Claim $23\left[\Gamma_{7}^{\bullet}, \Gamma_{4^{\prime}}^{\bullet} \Gamma_{4}^{\bullet} \Gamma_{3^{\prime}}^{\bullet} \Gamma_{4}^{\bullet-1} \Gamma_{4^{\prime}}^{\bullet-1}\right]=1$.

Thus $\left[\Gamma_{4^{\prime}}^{\bullet-1} \Gamma_{7}^{\bullet} \Gamma_{4^{\prime}}^{\bullet}, \Gamma_{4}^{\bullet} \Gamma_{3^{\prime}}^{\bullet} \Gamma_{4}^{\bullet^{-1}}\right]=1$.

From Claim 27, Claim 20 and Claim II.4(a) $\quad\left[\Gamma_{7}^{\bullet} \Gamma_{4^{\prime}}^{\bullet} \Gamma_{7}^{\bullet-1}, \Gamma_{3^{\prime}}^{\bullet-1} \Gamma_{4}^{\bullet} \Gamma_{3^{\prime}}^{\bullet}\right]=1$.

By Claim $22\left[\Gamma_{7}^{\bullet} \Gamma_{4^{\prime}}^{\bullet} \Gamma_{7}^{\bullet^{-1}}, \Gamma_{7}^{\bullet} \Gamma_{5}^{\bullet} \Gamma_{7}^{\bullet^{-1}}\right]=1$.

Thus, $\left[\Gamma_{4^{\prime}}^{\bullet}, \Gamma_{5}^{\bullet}\right]=1$.

Since $\Gamma_{4^{\prime}}^{\bullet}=\Gamma_{4^{\prime}}, \Gamma_{5}^{\bullet}=\Gamma_{5}$ we get the Claim.

Proof of Claim 29.

By Claim $13 \Gamma_{6^{\prime}}=\Gamma_{4}^{-1} \Gamma_{4^{\prime}}^{-1} \Gamma_{6} \Gamma_{4^{\prime}} \Gamma_{4}$.

Thus, $\Gamma_{4} \Gamma_{6^{\prime}} \Gamma_{4}^{-1}=\Gamma_{4^{\prime}}^{-1} \Gamma_{6} \Gamma_{4^{\prime}}$.

By Claim 1 and Claim II, 4(a)

$$
\Gamma_{6^{\prime}}^{-1} \Gamma_{4} \Gamma_{6^{\prime}}=\Gamma_{6} \Gamma_{4^{\prime}} \Gamma_{6}^{-1}
$$

Thus,

$$
\Gamma_{4}=\Gamma_{6^{\prime}} \Gamma_{6} \Gamma_{4^{\prime}} \Gamma_{6}^{-1} \Gamma_{6^{\prime}}^{-1} .
$$

We substitute the last equation in Claim 29 to get

$$
\left[\Gamma_{6^{\prime}} \Gamma_{6} \Gamma_{4^{\prime}} \Gamma_{6}^{-1} \Gamma_{6^{\prime}}^{-1}, \Gamma_{5}\right]=1 .
$$

By Claim $9\left[\Gamma_{5}, \Gamma_{6}\right]=1$. Thus,

$$
\left[\Gamma_{4^{\prime}}, \Gamma_{5}\right]=1
$$


Proof of Claim 30. By Claim 28, Claim 29 and Claim II.5(a).

Proof of Claim 31. We apply $Z_{44^{\prime}} Z_{55^{\prime}}$ on $\left[\Gamma_{4^{\prime}}, \Gamma_{5}\right]=1$ and on $\left[\Gamma_{4}, \Gamma_{5}\right]=1$ to get $\left[\Gamma_{4^{\prime}} \Gamma_{4} \Gamma_{4^{\prime}}^{-1}, \Gamma_{5^{\prime}}\right]=\left[\Gamma_{4^{\prime}}, \Gamma_{5^{\prime}}\right]=1$. We then use Lemma II.5(b) to get $\left[\Gamma_{4}, \Gamma_{5^{\prime}}\right]=1$. Together with Claim 30 , we get the Claim.

Proof of Claim 32. By Claim 7 and Claim 10.

Proof of Claim 33.

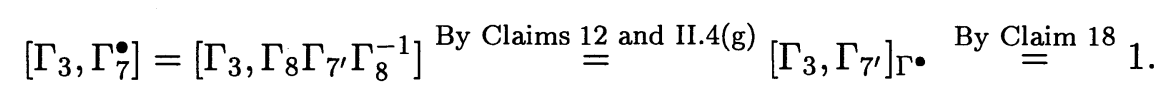

Proof of Claim 34.

$\left[\Gamma_{7}^{\bullet}, \Gamma_{5}^{2} \Gamma_{3} \Gamma_{5}^{-2}\right] \stackrel{\text { Claims } 10 \text { and } 32}{=}\left[\Gamma_{7}^{\bullet}, \Gamma_{5}^{2} \Gamma_{2^{\prime}}^{-1} \Gamma_{3} \Gamma_{2^{\prime}} \Gamma_{5}^{-2}\right] \stackrel{\text { Claim } 3}{=}\left[\Gamma_{7}^{\bullet}, \Gamma_{5}^{2} \Gamma_{3^{\prime}}^{\bullet} \Gamma_{5}^{-2}\right]$.

Thus, $\left[\Gamma_{7}^{\bullet}, \Gamma_{5}^{2} \Gamma_{3^{\prime}}^{\bullet} \Gamma_{5}^{-2}\right] \Leftrightarrow\left[\Gamma_{5^{-1}} \Gamma_{7}^{\circ} \Gamma_{5}, \Gamma_{5} \Gamma_{3^{\prime}}^{*} \Gamma_{5}^{-1}\right]=1$.

Now:

$$
\begin{aligned}
& {\left[\Gamma_{5}^{-1} \Gamma_{7}^{\bullet} \Gamma_{5}, \Gamma_{5} \Gamma_{3^{\prime}}^{\bullet} \Gamma_{5}^{-1}\right]} \\
& \stackrel{\text { Claim } 9}{=}\left[\Gamma_{5}^{\bullet^{-1}} \Gamma_{7}^{\bullet} \Gamma_{5}^{\bullet^{-1}}, \Gamma_{5}^{\bullet} \Gamma_{3^{\prime}}^{\bullet} \Gamma_{5}^{\bullet-1}\right] \\
& \text { Claims 21, 24, and II.4(a) }\left[\Gamma_{7}^{\circ} \Gamma_{5}^{\circ} \Gamma_{7}^{\circ-1}, \Gamma_{3^{\prime}}^{\circ-1} \Gamma_{5}^{\circ} \Gamma_{3^{\prime}}^{\circ}\right]= \\
& \stackrel{\text { Claim }}{=}{ }^{22}\left[\Gamma_{3^{\prime}}^{\bullet-1} \Gamma_{4}^{\bullet} \Gamma_{3^{\prime}}^{\bullet}, \Gamma_{3^{\prime}}^{\bullet-1} \Gamma_{5}^{\bullet} \Gamma_{3^{\prime}}^{\bullet}\right]=1 \\
& \stackrel{\text { Claim II.4(f) }}{=}\left[\Gamma_{4}^{*}, \Gamma_{5}^{*}\right]_{\Gamma_{3^{\prime}}}=1 \\
& \stackrel{\text { Claim }}{=}\left[\Gamma_{4}, \Gamma_{5}\right]_{\Gamma_{3^{\prime}}} \text { • } \\
& \text { Claim }{ }^{29} 1 \text {. }
\end{aligned}
$$

Proof of Claim 35. Let $f: B_{5} \rightarrow G$ be as follows:

$B_{5}=\left\langle X_{1}, \ldots, X_{4}\left|\left[X_{i}, X_{j}\right]=1\right| i-j \mid>2,\left\langle X_{i}, X_{i+1}\right\rangle=1, i=1,2,3\right\rangle$. $f\left(X_{1}\right)=\Gamma_{2^{\prime}}, f\left(X_{2}\right)=\Gamma_{3}, f\left(X_{3}\right)=\Gamma_{5}, f\left(X_{4}\right)=\Gamma_{7}^{\circ}$. 
By Claims 10, 32, 33, 2, 26, 21, $f$ is well-defined.

Let $d$ be the braid that satisfies $\left(X_{i}\right)_{d}=X_{5-i}, i=1,2,3$.

Then

$$
\begin{aligned}
1 & =\left[\Gamma_{7}^{\bullet}, \Gamma_{5}^{2} \Gamma_{3} \Gamma_{5}^{-2}\right]=f\left[X_{4}, X_{3}^{2} X_{2} X_{3}^{-2}\right] \\
& =f\left[\left(X_{1}\right)_{d},\left(X_{2}\right)_{d}^{2}\left(X_{3}\right)_{d}\left(X_{2}\right)_{d}^{-2}\right] \\
& =f\left(\left[X_{1}, X_{2}^{2} X_{3} X_{2}^{-2}\right]_{d}\right) \\
& =\left[f\left(X_{1}\right), f\left(X_{2}\right)^{2} f\left(X_{3}\right) f\left(X_{2}\right)^{-2}\right]_{f(d)}
\end{aligned}
$$

Thus, $\left[f\left(X_{1}\right), f\left(X_{2}\right)^{2} f\left(X_{3}\right) f\left(X_{2}^{-2}\right]=1\right.$.

Thus, $\left[\Gamma_{2^{\prime}}, \Gamma_{3}^{2} \Gamma_{5} \Gamma_{3}^{-2}\right]=1$.

Proof of Claim 36.

$\left[\Gamma_{3}, \Gamma_{4}\right] \stackrel{\text { Claim } 9}{=}\left[\Gamma_{3}, \Gamma_{4}^{\bullet}\right] \stackrel{\text { Claim } 22}{=}\left[\Gamma_{3}, \Gamma_{3^{\prime}}^{\bullet} \Gamma_{7}^{\bullet} \Gamma_{5}^{\bullet} \Gamma_{7}^{\bullet^{-1}} \Gamma_{3^{\prime}}^{\bullet-1}\right]$.

Thus,

$$
\begin{aligned}
& {\left[\Gamma_{3}, \Gamma_{4}\right]=1} \\
& \Leftrightarrow\left[\Gamma_{3}, \Gamma_{3^{\prime}}^{\bullet} \Gamma_{7}^{\bullet} \Gamma_{5}^{\bullet} \Gamma_{7}^{\bullet-1} \Gamma_{3^{\prime}}^{\bullet^{-1}}\right]=1 \\
& \text { Claims } \stackrel{18}{\Leftrightarrow} \text { and } 19\left[\Gamma_{3}, \Gamma_{3^{\prime}}^{\bullet} \Gamma_{5}^{\bullet} \Gamma_{3^{\prime}}^{\bullet^{-1}}\right]=1 \\
& \Leftrightarrow\left[\Gamma_{3}, \Gamma_{2^{\prime}}^{-1} \Gamma_{3} \Gamma_{2^{\prime}} \Gamma_{5} \Gamma_{2^{\prime}}^{-1} \Gamma_{3}^{-1} \Gamma_{2^{\prime}}\right]=1 \\
& \Leftrightarrow\left[\Gamma_{2^{\prime}} \Gamma_{3} \Gamma_{2^{\prime}}^{-1}, \Gamma_{3} \Gamma_{2^{\prime}} \Gamma_{5} \Gamma_{2^{\prime}}^{-1} \Gamma_{3}^{-1}\right]=1 \\
& \text { Claim } 10\left[\Gamma_{2^{\prime}} \Gamma_{3} \Gamma_{2^{\prime}}^{-1}, \Gamma_{3} \Gamma_{5} \Gamma_{3}^{-1}\right]=1 \\
& \text { Claims } 2 \text { and II.4(a) }\left[\Gamma_{3}^{-1} \Gamma_{2^{\prime}} \Gamma_{3}, \Gamma_{3} \Gamma_{5} \Gamma_{3}^{-1}\right]=1 \\
& \Leftrightarrow\left[\Gamma_{2^{\prime}}, \Gamma_{3}^{2} \Gamma_{5} \Gamma_{3}^{-2}\right]=1
\end{aligned}
$$

which is true by Claim 35.

We get $\left[\Gamma_{\underline{3}}, \Gamma_{4}\right]$ by Corollary I.5.

Proof of Claim 37. Similar to Claim 34.

Proof of Claim 38. Claim 18, Claim 10, Claim 12, Claim 3. 
Proof of Claim 39. Let $f: B_{5} \rightarrow G$ be as follows:

$$
f\left(X_{1}\right)=\Gamma_{8}, f\left(X_{2}\right)=\Gamma_{7^{\prime}}, f\left(X_{3}\right)=\Gamma_{4}, f\left(X_{4}\right)=\Gamma_{3^{\prime}}^{\bullet} .
$$

By Claim 12, Claim 38, Claim 5, Claim 27, Claim 20, $f$ is well defined. Let $d$ be a braid such that $\left(X_{i}\right)_{d}=X_{4-i}, i=1,2,3,4$.

Now:

$$
\begin{aligned}
1=\left[\Gamma_{7^{\prime}}, \Gamma_{4}^{2} \Gamma_{3^{\prime}}^{\bullet} \Gamma_{4}^{-2}\right] & =\left[f\left(X_{2}\right), f\left(X_{3}\right)^{2} f\left(X_{4}\right) f\left(X_{3}\right)^{-2}\right] \\
& =f\left[X_{2}, X_{3}^{2} X_{4} X_{3}^{-2}\right] \\
& =f\left[\left(X_{3}\right)_{d},\left(X_{2}\right)_{d}^{2}\left(X_{1}\right)_{d}\left(X_{2}\right)_{d}^{-2}\right] \\
& =\left[f\left(X_{3}\right), f\left(X_{2}\right)^{2} f\left(X_{1}\right) f\left(X_{2}\right)^{-2}\right]_{f(d)} \\
& =\left[\Gamma_{4}, \Gamma_{7^{\prime}}^{2} \Gamma_{8} \Gamma_{7^{\prime}}^{-2}\right]_{f(d)} .
\end{aligned}
$$

Thus, $\left[\Gamma_{4}, \Gamma_{7^{\prime}}^{2} \Gamma_{8} \Gamma_{7^{\prime}}^{-2}\right]=1$.

Proof of Claim 40. Follows from Claim 30, like in Claim 36.

Proof of Claim 41. Apply $\left(Z_{44^{\prime}} Z_{55^{\prime}}\right)^{m_{4}}\left(Z_{77^{\prime}} Z_{33^{\prime}}\right)^{m_{3}}$ on $\left[\Gamma_{5}, \Gamma_{7^{\prime}}\right]=1$ from Claim 40.

Proof of Claim 42. In $H_{4}^{\prime}$ there is $\bar{Z}_{2^{\prime}, 44^{\prime}}^{(3)}$. We take its complex conjugate $\underline{Z}_{2^{\prime}, 44^{\prime}}^{(3)}$ and apply Corollary I.4 on $\underline{Z}_{2^{\prime}, 4}^{(3)}$ to get $\left\langle\Gamma_{2^{\prime}}, \Gamma_{4}\right\rangle=1$ and then apply Corollary I.5 to get $\left\langle\Gamma_{\underline{2}}, \Gamma_{\underline{4}}\right\rangle=1$.

Proof of Claim 43. We apply Corollary I.5 on Claims 2 and 5.

Proof of Claim 44. Similar to Claim 42.

Proof of Claim 45. By Claim 22, $\Gamma_{7}^{\bullet} \Gamma_{5}^{\bullet} \Gamma_{7}^{\bullet^{-1}}=\Gamma_{3^{\prime}}^{\bullet-1} \Gamma_{4}^{\bullet} \Gamma_{3^{\prime}}^{\bullet}$. Since $\left\langle\Gamma_{5}^{\bullet}, \Gamma_{7}^{\bullet}\right\rangle=1$ we have

$$
\Gamma_{5}^{\bullet-1} \Gamma_{7}^{\bullet} \Gamma_{5}^{\bullet}=\Gamma_{3^{\prime}}^{\bullet-1} \Gamma_{4}^{\bullet} \Gamma_{3^{\prime}}^{\bullet}
$$

Thus, $\Gamma_{7}^{\bullet}=\Gamma_{5}^{\bullet} \Gamma_{3^{\prime}}^{\bullet-1} \Gamma_{4}^{\bullet} \Gamma_{3^{\prime}}^{\bullet} \Gamma_{5}^{\bullet^{-1}}$. 
We substitute the formulas for $\Gamma_{3^{\prime}}^{\bullet}$ and $\Gamma_{7}^{\bullet}$ to get

$$
\Gamma_{7^{\prime}}^{-1} \Gamma_{8} \Gamma_{7^{\prime}}=\Gamma_{5} \Gamma_{2^{\prime}}^{-1} \Gamma_{3}^{-1} \Gamma_{2^{\prime}} \Gamma_{4} \Gamma_{2^{\prime}}^{-1} \Gamma_{3} \Gamma_{2^{\prime}} \Gamma_{5}^{-1} .
$$

Since $\left\langle\Gamma_{2^{\prime}}, \Gamma_{4}\right\rangle=1($ Claim 42$)$

$$
\Gamma_{7^{\prime}}^{-1} \Gamma_{8} \Gamma_{7^{\prime}}=\Gamma_{5} \Gamma_{2^{\prime}}^{-1} \Gamma_{3}^{-1} \Gamma_{4}^{-1} \Gamma_{2^{\prime}} \Gamma_{4} \Gamma_{3} \Gamma_{2^{\prime}}^{-1} \Gamma_{5}^{-1} .
$$

Since $\left[\Gamma_{5}, \Gamma_{3^{\prime}}\right]=1$

$$
\Gamma_{7^{\prime}}^{-1} \Gamma_{8} \Gamma_{7^{\prime}}=\Gamma_{2^{\prime}}^{-1} \Gamma_{5} \Gamma_{3}^{-1} \Gamma_{4}^{-1} \Gamma_{2^{\prime}} \Gamma_{4} \Gamma_{3} \Gamma_{5}^{-1} \Gamma_{2^{\prime}} .
$$

Since $\left[\Gamma_{2^{\prime}}, \Gamma_{8}\right]=\left[\Gamma_{2^{\prime}}, \Gamma_{7^{\prime}}\right]=1$

$$
\Gamma_{7^{\prime}}^{-1} \Gamma_{8} \Gamma_{7^{\prime}}=\Gamma_{5} \Gamma_{3}^{-1} \Gamma_{4}^{-1} \Gamma_{2^{\prime}} \Gamma_{4} \Gamma_{3} \Gamma_{5}^{-1}
$$

and

$$
\Gamma_{8}=\Gamma_{7^{\prime}} \Gamma_{5} \Gamma_{3}^{-1} \Gamma_{4}^{-1} \Gamma_{2^{\prime}} \Gamma_{4} \Gamma_{3} \Gamma_{5}^{-1} \Gamma_{7^{\prime}}^{-1}
$$

Proof of Claim 46. By $\tilde{Z}_{22^{\prime}}$ of $H_{4}^{\prime}$ we have:

$$
\Gamma_{2}=\Gamma_{4^{\prime}} \Gamma_{4} \Gamma_{3^{\prime}} \Gamma_{3} \Gamma_{2^{\prime}} \Gamma_{3}^{-1} \Gamma_{3^{\prime}}^{-1} \Gamma_{4}^{-1} \Gamma_{4^{\prime}}^{-1}
$$

We apply on it $Z_{88^{\prime}} Z_{22^{\prime}}$ using Corollary I.5 to get (recall that $\left(\Gamma_{2}\right)$ $Z_{22^{\prime}}^{\prime} Z_{88^{\prime}}=\Gamma_{2^{\prime}}$ and $\left.\left(\Gamma_{2}^{\prime}\right) Z_{22^{\prime}} Z_{88^{\prime}}=\left(\Gamma_{2}^{\prime}\right) Z_{22^{\prime}}=\Gamma_{2^{\prime}} \Gamma_{2} \Gamma_{2^{\prime}}^{-1}\right)$

$$
\Gamma_{2^{\prime}}=\Gamma_{4^{\prime}} \Gamma_{4} \Gamma_{3^{\prime}} \Gamma_{3} \Gamma_{2^{\prime}} \Gamma_{2} \Gamma_{2^{\prime}}^{-1} \Gamma_{3}^{-1} \Gamma_{3^{\prime}}^{-1} \Gamma_{4}^{-1} \Gamma_{4^{\prime}}^{-1}
$$

Proof of Claim 4\%. By $\tilde{Z}_{88^{\prime}}$ of $H_{4}^{\prime}$ we get, using the Van Kampen Theorem:

$$
\Gamma_{8^{\prime}}=\Gamma_{5}^{-1} \Gamma_{5^{\prime}}^{-1} \Gamma_{7}^{-1} \Gamma_{7^{\prime}}^{-1} \Gamma_{8} \Gamma_{7} \Gamma_{7} \Gamma_{5^{\prime}} \Gamma_{5}
$$

Proof of Claim 48.

In $C_{5}^{\prime}$ we have $\tilde{Z}_{i 9}^{2}=H\left(\tilde{z}_{i 9}\right)^{2}$ for $i=1,2,3,4,6,7$, where: 


$$
\left.\tilde{z}_{i 9}=\underset{\left(8,8^{\prime}\right)}{\overline{\bar{s}}}\right)_{i 9}
$$

Case 1. $\quad i \neq 7$

The complex conjugation of $\tilde{Z}_{i 9}$ is $\overline{\tilde{Z}}_{i 9}=H\left(\overline{\tilde{z}}_{i 9}\right)$ and

$\overline{\tilde{z}}_{i 9}={\left.\stackrel{\left(8,8^{\prime}\right)}{z}\right)}_{i 9}$ which implies on $G($ as in Claim 0$) \quad\left[\Gamma_{i}, \Gamma_{8}^{-1} \Gamma_{8^{\prime}}^{-1} \Gamma_{9} \Gamma_{8^{\prime}} \Gamma_{8}\right]=1$.

By Claim 10, for $i=2,3,4$ we have $\left[\Gamma_{i}, \Gamma_{\underline{8}}\right]=1$.

By Claim 0 , for $i=1,6$ we have $\left[\Gamma_{i}, \Gamma_{8}\right]=1$.

Thus, $\left[\Gamma_{i}, \Gamma_{9}\right]=1$. We use Corollary I.5 to get $\left[\Gamma_{\underline{i}}, \Gamma_{\underline{9}}\right]=1$.

Case 2. $\quad i=7$

In $C_{5}^{\prime}$ we have $\underline{Z}_{7,9}^{2}$ which implies $\left[\Gamma_{7}, \Gamma_{9}\right]=1$ and thus, by Corollary I.7, $\left[\Gamma_{\underline{7}}, \Gamma_{\underline{g}}\right]=1$.

From Case 1 and Case 2 we get Claim 48.

Thus we have proved all the Claims.

We shall now prove the statements of the Proposition. We use the above claims, the definition of $E_{i}, E_{i^{\prime}}$ and the facts $\left\{\Gamma_{\underline{i}}\right\}=\left\{E_{\underline{i}}\right\}$ and $E_{i^{\prime}} E_{i}=$ $\Gamma_{i^{\prime}} \Gamma_{i} \forall i$.

(1) From Claim 1, Claim 26, Claim 27, Claim 42, Claim 43, Claim 44.

(2) From Claim 0, Claim 10, Claim 12, Claim 18, Claim 31, Claim 36, Claim 41, Claim 48.

(3) From Claim 45, definifions of $E_{i}$ and Corollary I.5.

(4) From Claim 46, definitions of $E_{i}$ and Corollary II.1.

(5) From Claim 47, definition of $E_{i}$ and the above fact.

(6) From Claim 13.

(7) From Claim 13.

Proposition II.6

We need the following corollary in order to obtain in Chapter IV, $\S 8$ a smaller set of generators for $G$.

Corollary II.7. Let $E_{i}, E_{i^{\prime}}$ be as in the beginning of the chapter. Let $A_{i}=E_{i^{\prime}} E_{i}^{-1}$. Then:

(1) $A_{5}=\left(A_{4}\right)_{E_{2}^{-1} E_{3} E_{7}^{-1} E_{8}}$ 
(2) $A_{7}=\left(A_{3}\right)_{E_{2}^{-1} E_{4} E_{5}^{-1} E_{7}^{-1}}$

(3) $A_{8}=\left(A_{2}\right)_{E_{4} E_{3} E_{5}^{-1} E_{7}^{-1}}$

(4) $A_{2}=E_{1}^{-2} A_{1}^{-1}(A)_{E_{2}^{-1}}\left(E_{1}^{2}\right)_{E_{2}^{-1}}$

(5) $A_{6}=E_{4}^{-2} A_{4}^{-1}\left(A_{4}\right)_{E_{6}^{-1}}\left(E_{4}^{2}\right)_{E_{6}^{-1}}$

(6) $A_{3}=E_{1}^{-2} A_{1}^{-1}(A)_{E_{3}^{-1}}\left(E_{1}^{2}\right)_{E_{3}^{-1}}$

(7) $A_{9}=E_{5}^{-2} A_{5}^{-1}\left(A_{5}\right)_{E_{9}^{-1}}\left(E_{5}^{2}\right)_{E_{9}^{-1}}$

(8) $\left(A_{4}\right)_{E_{2}^{-1} E_{4}^{-1}}=E_{4}^{2} A_{3} E_{3}^{2} A_{2}\left(E_{3}^{-2}\right)_{E_{2}^{-1}}\left(A_{3}^{-1}\right)_{E_{2}^{-1}}\left(E_{4}^{-2}\right)_{E_{2}^{-1}}$

(9) $\left(A_{7}\right)_{E_{6}^{-1} E_{7}^{-1}}=E_{7}^{2} A_{6}\left(E_{7}^{-2}\right)_{E_{6}^{-1}}$

(10) $\left(A_{9}\right)_{E_{8}^{-1} E_{9}^{-1}}=E_{9}^{2} A_{8}\left(E_{9}^{-2}\right)_{E_{8}^{-1}}$

Proof. We use proposition II.6 (1), ., (7). The claims are grouped according to the similarity of their proofs and not according to the order that we use them in Proposition IV.8.1.

Recall:

$$
\begin{aligned}
\left(E_{i}\right) \rho_{i} & =E_{i^{-1}} \\
\left(E_{i^{\prime}}\right) \rho_{i} & =E_{i^{\prime}} E_{i} E_{i^{\prime}} \\
E_{i^{\prime}} & =A_{i} E_{i} \\
E_{i^{\prime}} E_{i} & =A_{i} E_{i}^{2} .
\end{aligned}
$$

For (1), (2), (3) we use (5) of Proposition II.6.

For Claims (4)-(7) we use (6) of Proposition II.6. The 4 claims are symmetric and we shall only prove the first one.

For (8) we use (4) of Proposition II.6.

For (9)-(10) we use (7) of Proposition II.6. The claims are symmetric and we shall only prove the first one.

(1) By (5) of Proposition II.6

$$
E_{8}=\left(E_{4}^{-1} E_{2} E_{4}\right)_{E_{3} E_{5}^{-1} E_{7}^{-1}}
$$

By (1), (2) and Claim II.4(a)

$$
E_{8}=\left(E_{2} E_{4} E_{2}^{-1}\right)_{E_{3} E_{7}^{-1} E_{5}^{-1}}
$$


Thus,

$$
E_{5}^{-1} E_{8} E_{5}=\left(E_{4}\right)_{E_{2}^{-1} E_{3} E_{7}^{-1}} .
$$

By (1) and Claim II.4(a)

$$
E_{8} E_{5} E_{8}^{-1}=\left(E_{4}\right)_{E_{2}^{-1} E_{3} E_{7}^{-1}} .
$$

Thus,

$$
E_{5}=\left(E_{4}\right)_{E_{2}^{-1} E_{3} E_{7}^{-1} E_{8}} .
$$

We apply on it $\rho_{4} \rho_{5}$ to get

$$
E_{5^{\prime}}=\left(E_{4^{\prime}}\right)_{E_{2}^{-1} E_{3} E_{7}^{-1} E_{8}} .
$$

We multiply the 2 results to get

$$
A_{5}=\left(A_{4}\right)_{E_{2}^{-1} E_{3} E_{7}^{-1} E_{8} .} .
$$

(2) By (5) of Proposition II.6

$$
E_{7}^{-1} E_{8} E_{7}=E_{5} E_{4}^{-1} E_{3}^{-1} E_{2} E_{3} E_{4} E_{5}^{-1} .
$$

By (1) and Claim II.4(a)

$$
E_{8} E_{7} E_{8}^{-1}=E_{4} E_{4}^{-1} E_{2} E_{3} E_{2}^{-1} E_{4} E_{5}^{-1} .
$$

Thus,

$$
E_{7}=\left(E_{9}\right)_{E_{2}^{-1} E_{4} E_{5}^{-1} E_{8}} .
$$

We apply on this $\rho_{2} \rho_{3}$ to get

$$
E_{7^{\prime}}=\left(E_{3^{\prime}}\right)_{E_{2}^{-1} E-4 E-5^{-1} E_{8}} .
$$

We multiply the results to get

$$
A_{7}=\left(A_{3}\right)_{E_{2}^{-1} E_{4} E_{5}^{-1} E_{8}} .
$$

(3) By (5) of Proposition II.6

$$
E_{8}=\left(E_{2}\right)_{E_{4} E_{3} E_{5}^{-1} E_{7}^{-1}} .
$$

We apply on it $\rho_{2} \rho_{8}$ to get

$$
E_{8^{\prime}}=\left(E_{2^{\prime}}\right)_{E_{4} E_{3} E_{5}^{-1} E_{7}^{-1} .}
$$

We multiply the 2 results to get

$$
A_{8}=\left(A_{2}\right)_{E_{4} E_{3} E_{5}^{-1} E_{7}} .
$$


(4) From (6) of Proposition II.6

$$
\begin{aligned}
& E_{2^{\prime}}=E_{1}^{-1} E_{1^{\prime}}^{-1} E-2 E_{1^{\prime}} E_{1} \\
& \Rightarrow A_{2} E_{2}=E_{1}^{-2} A_{1}^{-1} E_{2} A_{1} E_{1}^{2} \\
& \Rightarrow A_{2}=E_{1}^{-2} A_{1}^{-1}\left(A_{1}\right)_{2}^{-1}\left(E_{1}^{2}\right)_{E_{2}^{-1}}
\end{aligned}
$$

(8) From (4) of Proposition II.6

$$
\begin{aligned}
& E_{2}=E_{4^{\prime}} E_{4} E_{3^{\prime}} E_{3} E_{2^{\prime}} E_{3}^{-1} E_{3^{\prime}}^{-1} E_{4}^{-1} E_{4^{\prime}}^{-1} \\
& \Rightarrow E_{2}=A_{4} E_{4}^{2} A_{3} E_{3}^{2} A_{2} E_{2} E_{3}^{-2} A_{3}^{-1} E_{4}^{-2} A_{4}^{-1} \\
& \Rightarrow A_{4}^{-1} E_{2} A_{4}=E_{4}^{2} A_{3} E_{3}^{2} A_{2} E_{2} E_{3}^{-2} A_{3}^{-1} E_{4}^{-2} \\
& \Rightarrow A_{4}^{-1}\left(A_{4}\right)_{E_{2}^{-1}}=E_{4}^{2} A_{3} E_{3}^{2} A_{2}\left(E_{3}^{-2} A_{3}^{-1} E_{4}^{-2}\right)_{E_{2}^{-1}} \\
& \Rightarrow A_{4}^{-1}\left(A_{4}\right)_{E_{2}^{-1}}=E_{4}^{2} A_{3} E_{3}^{2} A_{2}\left(E_{3}^{-2}\right)_{E_{2}^{-1}}\left(A_{3}^{-1}\right)_{E_{2}^{-1}}\left(E_{4}^{-2}\right)_{E_{2}^{-1}} .
\end{aligned}
$$

By Lemma II.4(h)

$$
\begin{aligned}
& \left(A_{4}\right)_{E_{2}^{-1} E_{4}^{-1}}=A_{4}^{-1}\left(A_{4}\right)_{E_{2}^{-1}} . \text { Thus, } \\
& \left(A_{4}\right)_{E_{2}^{-1} E_{4}^{-1}}=E_{4}^{2} A_{3} E_{3}^{2} A_{2}\left(E_{3}^{-2}\right)_{E_{2}^{-1}}\left(A_{3}^{-1}\right)_{E_{2}^{-1}}\left(E_{4}^{-2}\right)_{E_{2}^{-1}} .
\end{aligned}
$$

(9) From (7)

$$
\begin{aligned}
& E_{6}=E_{7^{\prime}} E_{7} E_{6^{\prime}} E_{7}^{-1} E_{7^{\prime}}^{-1} \\
& \Rightarrow E_{6}=A_{7} E_{7}^{2} A_{6} E_{6} E_{7}^{-2} A_{7}^{-1} \\
& \Rightarrow A_{7}^{-1} E-6 A_{7}=E_{7}^{2} A_{6} E_{6} E_{7}^{-2} \\
& \Rightarrow A_{7}^{-1}\left(A_{7}\right)_{E_{6}^{-1}}=E_{7}^{2} A_{6}\left(E_{7}^{-2}\right)_{E_{6}^{-1}} .
\end{aligned}
$$

By Lemma II.4(h), $A_{7}^{-1}\left(A_{7}\right)_{E_{6}^{-1}}=\left(A_{7}\right)_{E_{6}^{-1} E_{7}^{-1}}$. Thus,

$$
\left(A_{7}\right)_{E_{6}^{-1} E_{7}^{-1}}=E_{7}^{2} A_{6}\left(E_{7}^{-2}\right)_{E_{6}^{-1}} \text {. }
$$

\section{Construction theory for $\tilde{B}_{n}$.}

Let $D$ be a disc, $K \subset D, \# K=n$. Let $B_{n}=B_{n}[D, K]$ (see definition in Chapter 0). 


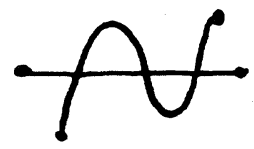

(l)

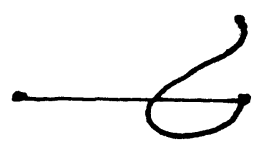

(iv)

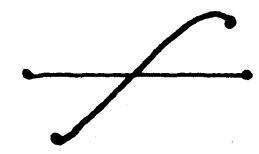

(ii)

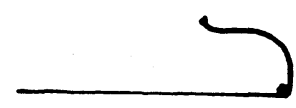

(v)

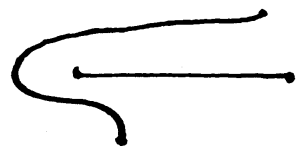

(iii)

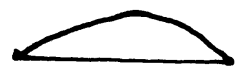

$(v i)$

Figure III.1.0.

\section{III.1. Definition of $\tilde{\mathbf{B}}_{\mathbf{n}}$.}

Definition. Let $D, K$ be as before. Let $H\left(\sigma_{1}\right)$ and $H\left(\sigma_{2}\right)$ be 2 half-twists in $B=B_{n}[D, K]$. We say that $H\left(\sigma_{1}\right)$ and $H\left(\sigma_{2}\right)$ are:

(i) weakly disjoint if $\sigma_{1} \cap \sigma_{2} \cap K=\emptyset$.

(ii) transversal if $\sigma_{1}$ and $\sigma_{2}$ are weakly disjoint and intersect each other exactly once (and not in any point of $K$ ), i.e., $\sigma_{1} \cap \sigma_{2}=$ one point, $\sigma_{1} \cap \sigma_{2} \cap K=\emptyset$.

(iii) disjoint if $\sigma_{1} \cap \sigma_{2}=\emptyset$.

(iv) adjacent if $\sigma_{1} \cap \sigma_{2} \cap K=$ one point.

(v) consecutive if they are adjacent and $\sigma_{1} \cap \sigma_{2}$ do not intersect outside of $K$, i.e., $\sigma_{1} \cap \sigma_{2}=$ point $\in K$.

(vi) $\underline{\text { cyclic }}$ if $\sigma_{1} \cap \sigma_{2}=2$ points $\in K$.

Claim III.1.0. Let $X, Y$ be 2 half-twists in $B_{n}$. Then: 

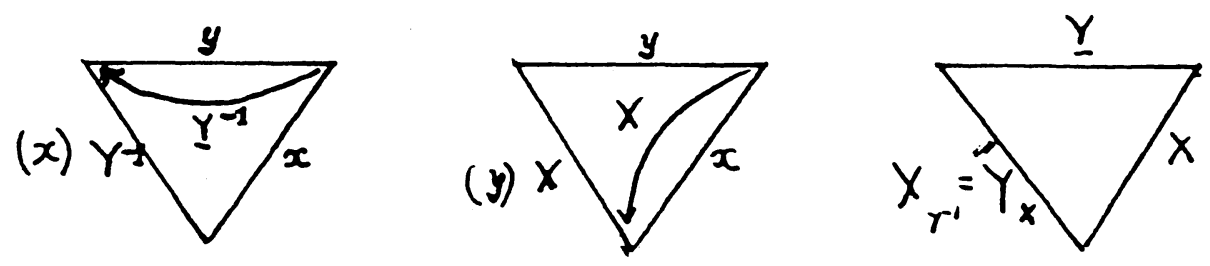

Figure III.1.1.

(i) If $X, Y$ are disjoint, then $[X, Y]=1$, i.e., $X Y=Y X$.

(ii) If $X, Y$ are consecutive, then $\langle X, Y\rangle=X Y X Y^{-1} X^{-1} Y^{-1}=1$ and $X Y X=Y X Y$ or $X_{Y^{-1}}=Y_{X}$. We say then that $X$ and $Y$ satisfy the triple relation.

(iii) If $X=H(x)=\bar{\beta}, Y=H(y)$, then $Y_{X}=X^{-1} Y X=H((y) \beta)$.

Proof. (i) (ii) Basic properties of a braid group. See, for example, Chapter 3 of [MoTe4].

(iii) See Fig. III.1.1 for a geometrical presentation of $Y_{X}$. The common point of $x$ and $y$ travels under $\beta$ counterclockwise to the other end of $x$. Thus (y) $\beta$ connects the 2 ends of $x$ and $y$, which is not a common end of either of them. By IV.3.4 of [MoTe12], $Y_{X}=X^{-1} Y X=(Y) X^{\vee}$ (see definition or $f^{\vee}$ in [MoTe4], Chapter 4) which is equal by Claim IV.3.0 of [MoTe12] to $H\left((y) \beta_{1}\right)$.

Definition. $\tilde{B}_{n}$

Let $\tilde{B}_{n}$ be the quotient of $b_{n}$, the Braid group of order $n$, by the subgroup generated by the commutators $\left[H\left(\sigma_{1}\right), H\left(\sigma_{2}\right)\right]$ where $H\left(\sigma_{1}\right)$ and $H\left(\sigma_{2}\right)$ are transversal half-twists.

\section{Notations.}

Let $Y \in B_{n}$. We denote the image of $Y$ in $\tilde{B}_{n}$. by $\tilde{Y}$. When possible, we shall abuse notation and denote $\tilde{Y}$ by $Y$. If $Y$ is a half-twist in $B_{n}$ we call 


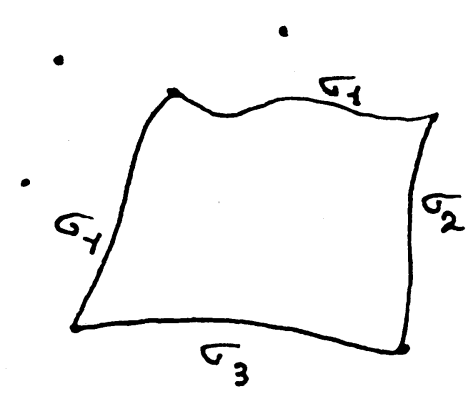

Figure III.1.2.

$\tilde{Y}$ a half-twist in $\tilde{B}_{n}$. We call two half-twists $\tilde{Y}, \tilde{X}$ in $\tilde{B}_{n}$ disjoint (or weakly disjoint, adjacent, consecutive, transversal) if $Y, X$ are disjoint (or weakly disjoint, adjacent, consecutive, transversal). If $\left\{X_{i}\right\}$ is a frame of $B_{n}$, then $\left\{\tilde{X}_{i}\right\}$ is a frame in $\tilde{B}_{n}$. We also refer to $\left\{\tilde{X}_{i}\right\}$ as a standard base of $\tilde{B}_{n}$.

Definition. Polarized half-twist, polarization

We say that a half-twist $X \in B_{n}$ (or $\tilde{X}$ in $\tilde{B}_{n}$ ) is polarized if we choose an order on the end points of $X$. The order is called the polarization of $X$ or $\tilde{X}$.

\section{Definition. Orderly adjacent}

Let $X, Y$ be two adjacent polarized half-twists in $B_{n}$ (resp. in $\tilde{B}_{n}$ ). We say that $X, Y$ are orderly adjacent if their common point is the "end" of one of them and the "origin" of another.

Definition. Good quadrangle

Let $H\left(\sigma_{i}\right) \quad i=1,2,3,4$ be 4 half-twists such that $H\left(\sigma_{i}\right)$ and $H\left(\sigma_{i+1}\right)$ are consecutive, $H\left(\sigma_{4}\right)$ and $H\left(\sigma_{1}\right)$ are consecutive, $H\left(\sigma_{1}\right)$ and $H\left(\sigma_{3}\right)$ are disjoint and $H\left(\sigma_{2}\right)$ and $H\left(\sigma_{4}\right)$ are disjoint, and in the interior of $\bigcup_{i=1}^{4} \sigma_{i}$ there is no point of $K$. We say that $\left\{H\left(\sigma_{i}\right)\right\}$ is a good quadrangle in $B_{n}$ and $\left\{\widetilde{H\left(\sigma_{i}\right)}\right\}$ is a good quadrangle in $\tilde{B}_{n}$.

Remark III.1.1. (a) transversal, disjoint $\Rightarrow$ weakly disjoint. consecutive $\Rightarrow$ adjacent.

(b) Any two pairs of disjoint (or transversal, consecutive, cyclic) halftwists are conjugate to each other by an element $b \in B$. 


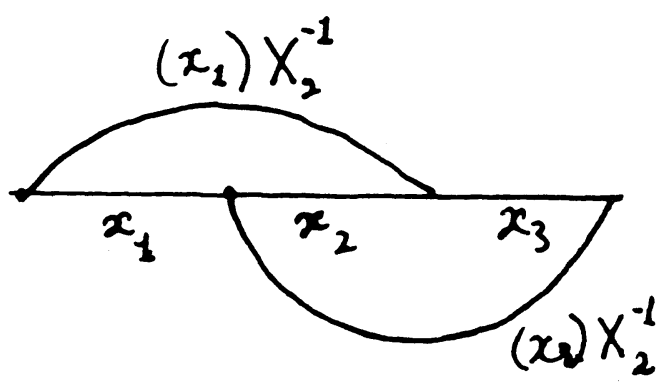

Figure III.1.3.

(c) Any two half-twists in $B_{n}$ (or $\tilde{B}_{n}$ ) are conjugate to each other by an element of $B_{n}$ (or $\tilde{B}_{n}$ ).

(d) Every 2 transversal or disjoint half-twists in $\tilde{B}_{n}$ commute. Every 2 consecutive half-twists in $B_{n}$ or in $\tilde{B}_{n}$ satisfy the triple relation $(X Y X=Y X Y)$.

(e) Any two good quadrangles in $B_{n}$ or in $\tilde{B}_{n}$ are conjugate.

(f) Every 2 pairs of orderly adjacent (non-orderly adjacent) consecutive half-twists are conjugate to each other by an element $b \in B$ preserving polarization.

Proof. Geometric observation in $B_{n}$ and $\tilde{B}_{n}$, and Claim III.1.0.

Lemma III.1.2. If $\left\{\tilde{Y}_{i}\right\} \quad i=1, \ldots, 4$ is a good quadrangle in $\tilde{B}_{n}$, then (a) $\tilde{Y}_{1} \tilde{Y}_{3}=\tilde{Y}_{3} \tilde{Y}_{1}, \quad$ (b) $\tilde{Y}_{1}^{2} \tilde{Y}_{3}^{3}=\tilde{Y}_{2}^{2} \tilde{Y}_{4}^{2}$.

Proof.

(a) Since they are disjoint.

(b) Let $X_{1}, X_{2}, X_{3}$ be 3 half-twists such that $X_{1}$ and $X_{2}$ are consecutive, $X_{2}$ and $X_{3}$ are consecutive and $X_{1}$ and $X_{3}$ are disjoint. Denote $X_{i}=H\left(x_{i}\right), \quad i=1, \ldots, 4$. Clearly, $X_{1},\left(X_{3}\right)_{X_{2}^{-1}}, X_{3},\left(X_{1}\right)_{X_{2}^{-1}}$ is a good quadrangle (see Fig. III.1.3).

In $B_{n}:\left[X_{1}, X_{3}\right]=1$ and $\left\langle X_{1}, X_{2}\right\rangle=\left\langle X_{3}, X_{2}\right\rangle=1$. Thus, we can use Claim II.4(a). 
It is clear that $\left(X_{2}\right)_{X_{1} X_{3}}$ is transversal to $X_{2}$. Thus, in $\tilde{B}_{n}$ :

$$
\left[\tilde{X}_{2},\left(\tilde{X}_{2}\right)_{\tilde{X}_{1} \tilde{X}_{3}}\right]=1
$$

Thus,

$$
\begin{aligned}
& 1=\left[\tilde{X}_{2}, \tilde{X}_{3}^{-1} \tilde{X}_{1}^{-1} \tilde{X}_{2} \tilde{X}_{1} \tilde{X}_{3}\right] \\
& =\tilde{X}_{2} \tilde{X}_{3}^{-1} \tilde{X}_{1}^{-1} \tilde{X}_{2} \tilde{X}_{1} \underbrace{\tilde{X}_{3} \tilde{X}_{2}^{-1} \tilde{X}_{3}^{-1}} \tilde{X}_{1}^{-1} \tilde{X}_{2}^{-1} \tilde{X}_{1} \tilde{X}_{3} \\
& \text { (By Claim II.4) }=\tilde{X}_{2} \tilde{X}_{3}^{-1} \tilde{X}_{1}^{-1} \tilde{X}_{2} \tilde{X}_{1} \tilde{X}_{2}^{-1} \tilde{X}_{3}^{-1} \underbrace{\tilde{X}_{2} \tilde{X}_{1}^{-1} \tilde{X}_{2}^{-1}} \tilde{X}_{1} \tilde{X}_{3} \\
& \text { (By Claim II.4) }=\quad \overbrace{\tilde{X}_{1}^{-1} \tilde{X}_{2}^{-1} \tilde{X}_{1}}^{-\tilde{X}_{1}} \tilde{X}_{3} \\
& \left(\left[X_{1}, X_{3}\right]=1\right)=\tilde{X}_{2} \tilde{X}_{3}^{-1} \tilde{X}_{1}^{-1} \tilde{X}_{2} \underbrace{\tilde{X}_{1} \tilde{X}_{2}^{-1} \tilde{X}_{1}^{-1}} \tilde{X}_{3}^{-1} \tilde{X}_{2}^{-1} \tilde{X}_{1} \tilde{X}_{1} \tilde{X}_{3}
\end{aligned}
$$

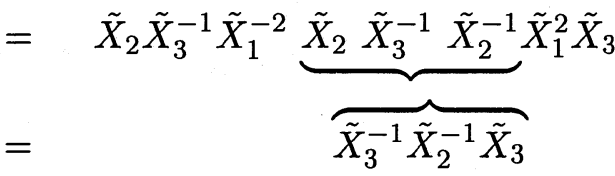

$$
\begin{aligned}
& \overbrace{\tilde{X}_{2}^{-1} \tilde{X}_{1}^{-1} \tilde{X}_{2}} \\
& \text { (By Claim II.4) }= \\
& \left(\text { Since }\left[X_{1}, X_{3}\right]=1\right)=\tilde{X}_{2} \tilde{X}_{3}^{-2} \tilde{X}_{1}^{-2} \tilde{X}_{2}^{-1} \tilde{X}_{1}^{2} \tilde{X}_{3}^{2} \\
& =\left(\tilde{X}_{3}\right)_{\tilde{X}_{2}^{-1}}^{-2}\left(\tilde{X}_{1}\right)_{\tilde{X}_{2}^{-1}}^{-2} \tilde{X}_{1}^{2} \tilde{X}_{3}^{2} .
\end{aligned}
$$

Thus, $\tilde{X}_{1}^{2} \tilde{X}_{3}^{2}=\left(\tilde{X}_{1}\right)_{\tilde{X}_{2}^{-1}}^{2} \cdot\left(\tilde{X}_{3}\right)_{\tilde{X}_{2}^{-1}}^{2}$.

By Remark III.1.1, $\left\{\tilde{Y}_{i}\right\}$ is conjugate to $\left\{\tilde{X}_{1},\left(\tilde{X}_{3}\right)_{\tilde{X}_{2}^{-1}}, \tilde{X}_{3},\left(\tilde{X}_{1}\right)_{\tilde{X}_{2}^{-1}}\right\}$ and thus satisfies $\tilde{Y}_{1}^{2} \tilde{Y}_{3}^{2}=\tilde{Y}_{4}^{2} \tilde{Y}_{2}^{2}$.

\section{III.2. $\tilde{\mathbf{B}}_{\mathrm{n}}$-groups and prime elements.}

Definition. $\tilde{B}_{n}-$ group.

A group $G$ is called a $\tilde{B}_{n}$-group if there exists a homomorphism $\tilde{B}_{n} \rightarrow$ $\operatorname{Aut}(G)$. We denote $(g)_{b}$ by $g_{b}$.

Definition. Prime element, supporting half-twist (s.h.t.) corresponding central element.

Let $G$ be a $\tilde{B}_{n}$-group. 
An element $g \in G$ is called a prime element of $G$ if there exists a halftwist $X \in B_{n}$ and $\tau \in \operatorname{Center}(G)$ with $\tau^{2}=1$ and $\tau_{b}=\tau \forall b \in \tilde{B}_{n}$ such that:

(1) $g_{\tilde{X}^{-1}}=g^{-1} \tau$.

(2) For every half-twist $Y$ adjacent to $X$ we have:

(a) $g_{\tilde{X} \tilde{Y}^{-1} \tilde{X}^{-1}}=g_{\tilde{X}}^{-1} g_{\tilde{X} \tilde{Y}^{-1}}$

(b) $g_{\tilde{Y}^{-1} \tilde{X}^{-1}}=g^{-1} g_{\tilde{Y}^{-1}}$.

(3) For every half-twist $Z$ disjoint from $X, g_{\tilde{Z}}=g$.

The half-twist $X$ (or $\tilde{X})$ is called the supporting half-twist of $g, \quad(X$ is the s.h.t. of $g$.)

The element $\tau$ is called the corresponding central element.

Lemma III.2.1. Let $G$ be a $\tilde{B}_{n}$-group.

Let $g$ be a prime element in $G$ with supporting half-twist $X$ and corresponding central element $\tau$. Then:

(1) $g_{\tilde{X}}=g_{\tilde{X}^{-1}}=g^{-1} \tau, \quad g_{\tilde{X}^{2}}=g$.

(2) $g_{\tilde{Y}^{-2}}=g \tau \quad \forall Y$ consecutive half-twist to $X$.

(3) $\left[g, g_{\tilde{Y}^{-1}}\right]=\tau \quad \forall Y$ consecutive half-twist to $X$.

Proof.

(1) $g_{\tilde{X}^{-2}}=\left(g_{\tilde{X}^{-1}}\right)_{\tilde{X}^{-1}}=\left(g^{-1} \tau\right)_{\tilde{X}^{-1}}=\left(g^{-1}\right)_{\tilde{X}^{-1}} \cdot=\left(g^{-1} \tau\right)^{-1} \tau=g \Rightarrow$ $g_{\tilde{X}}=g_{\tilde{X}^{-1}} \stackrel{\text { Axiom }}{=}{ }^{(1)} g^{-1} \tau$.

(2) $g_{\tilde{X}^{-1} \tilde{Y}^{-1} \tilde{X}^{-1}}=\left(g_{\tilde{X}^{-1}}\right)_{\tilde{Y}^{-1} \tilde{X}^{-1}}=\left(g^{-1} \tau\right)_{\tilde{Y}^{-1} \tilde{X}^{-1}}=\left(g_{\tilde{Y}^{-1} \tilde{X}^{-1}}\right)^{-1}$. $\tau \stackrel{\text { Axiom }}{=}(2) g_{\tilde{Y}^{-1}}^{-1} \cdot g \cdot \tau$.

On the other hand:

$$
\begin{aligned}
g_{\tilde{X}^{-1} \tilde{Y}^{-1} \tilde{X}^{-1}} & =g_{\tilde{Y}^{-1} \tilde{X}^{-1} \tilde{Y}^{-1}}=\left(g_{\tilde{Y}^{-1} \tilde{X}^{-1}}\right)_{\tilde{Y}^{-1}} \stackrel{\text { Axiom }^{(2)}}{=}\left(g^{-1} g_{\tilde{Y}^{-1}}\right)_{\tilde{Y}^{-1}} \\
& =g_{\tilde{Y}}^{-1} \cdot g_{\tilde{Y}^{-2}} .
\end{aligned}
$$

Thus, $g_{\tilde{Y}^{-} 2}=g \tau$. 


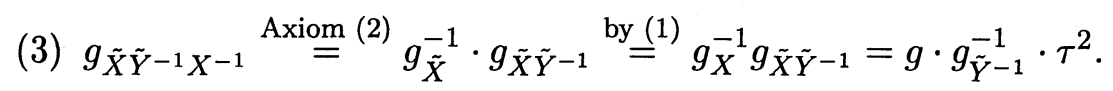

On the other hand:

$$
\begin{aligned}
& g_{\tilde{X} \tilde{Y}^{-1} \tilde{X}^{-1}} \stackrel{\text { by }(1)}{=}\left(g^{-1} \tau\right)_{\tilde{Y}^{-1} \tilde{X}^{-1}} \\
& \stackrel{\text { Axiom }}{=}{ }^{(2)}\left(g^{-1} \cdot g_{\tilde{Y}^{-1}}\right)^{-1} \cdot \tau=g_{\tilde{Y}^{-1}}^{-1} \cdot g \cdot \tau \text {. }
\end{aligned}
$$

Thus, $g \cdot g_{\tilde{Y}^{-1}}^{-1} \cdot=g_{\tilde{Y}^{-1}}^{-1} \cdot g \tau$.

Thus, $g_{\tilde{Y}^{-1}} \cdot g \cdot g_{\tilde{Y}^{-1}} \cdot g^{-1}=\tau^{-1}=\tau$.

Thus, $\left[g_{\tilde{Y}-1}, g\right]=\tau$.

Lemma III.2.2. Let $G$ be a $\tilde{B}_{n}$-group. Let $g$ be a prime element in $G$ with s.h.t. $X$ and corresponding central element $\tau$. Let $b \in \tilde{B}_{n}$. Then $g_{b}$ is a prime element with s.h.t. $X_{b}$ and central element $\tau$.

Proof. We use the fact that $\left(a_{b}\right)_{c}=\left(a_{c}\right)_{b_{c}}$ and $(a b)_{c}=a_{c} b_{c}$. We have to prove 3 properties:

(1) $g_{\tilde{X}^{-1}}=g^{-1} \tau \Rightarrow\left(g_{\tilde{X}^{-1}}\right)_{b}=\left(g^{-1} \tau\right)_{b} \Rightarrow g_{\tilde{X}^{-1} b}=g_{b}^{-1} \tau \Rightarrow g_{b b^{-1} \tilde{X}^{-1} b}=$ $\left(g_{b}\right)^{-1} \tau \Rightarrow\left(g_{b}\right)_{\tilde{X}_{b}^{-1}}=\left(g_{b}\right)^{-1} \cdot \tau$.

(2) Let $Y$ be a half-twist adjacent to $X_{b}$. Then $Y_{b^{-1}}$ is adjacent to $X$ and satisfies axiom (2) of prime elements for $g, X$ and $Y_{b}^{-1}$. Namely: $g_{\tilde{Y}^{-1}-1}^{-1} \tilde{X}=g^{-1} g_{\tilde{Y}_{b^{-1}}^{-1}}$ and $g_{\tilde{X} \tilde{Y}_{b^{-1}}^{-1} \tilde{X}^{-1}}=g_{\tilde{X}}^{-1} g_{\tilde{X} \tilde{Y}_{b^{-1}}^{-1}}$.

$$
\begin{aligned}
& \text { (a) } g_{\tilde{Y}_{b^{-1}}^{-1} \tilde{X}}=g^{-1} g_{\tilde{Y}_{b^{-1}}^{-1}} \Rightarrow \\
& \left(g_{\tilde{T}^{-1}-1} \tilde{X}_{b}=\left(g^{-1} g_{\tilde{Y}_{b^{-1}}^{-1}}\right)_{b} \Rightarrow\right. \\
& \left(g_{b}\right)_{\left(\tilde{Y}_{b^{-1}} \tilde{X}\right)_{b}}=\left(g_{b}\right)^{-1}\left(g_{b}\right)_{\tilde{Y}^{-1}} \Rightarrow \\
& \left(g_{b}\right)_{\tilde{Y} \tilde{X}_{b}}=g_{b}^{-1} \cdot\left(g_{b}\right)_{\tilde{Y}^{-1}} . \\
& \text { (b) } g_{\tilde{X} \tilde{Y}_{b^{-1}}^{-1} \tilde{X}^{-1}}=g_{\tilde{X}}^{-1} g_{\tilde{X} \tilde{Y}_{b^{-1}}^{-1}} \Rightarrow \\
& \left(g_{b}\right)_{\tilde{X}_{b} \tilde{Y}^{-1} \tilde{X}_{b}^{-1}}=\left(g_{b}^{-1}\right)_{\tilde{X}_{b}}\left(g_{b}\right)_{\tilde{X}_{b} \tilde{Y}^{-1}} .
\end{aligned}
$$

(3) Let $Z$ be a half-twist disjoint from $X_{b}$. Then $Z_{b^{-1}}$ is disjoint from $X$. Then $g_{\tilde{Z}_{b^{-1}}}=g$. We conjugate $g_{\tilde{Z}_{b}^{-1}}=g$ by $b$ by get: $\left(g_{b}\right)_{\tilde{Z}}=g_{b}$.

We need the following lemma on $B_{n}$ to prove later a criterion for prime element in a $\tilde{B}_{n}$-group. 
Claim III.2.3. Let $\left(X_{1}, \ldots, X_{n-1}\right)$ be a frame in $B_{n}=B_{n}[D, K]$. Let

$$
C\left(X_{1}\right)=\left\{b \in B_{n} \mid\left[b, X_{1}\right]=1\right\}
$$

(centralizer of $X_{1}$ ),

$$
C_{p}\left(X_{1}\right)=\left\{b \in B_{n} \mid\left(X_{1}\right)_{b}=X_{1} \text {, preserving polarization }\right\} .
$$

Let $\sigma=X_{2} X_{1}^{2} X_{2}$. Then $C\left(X_{1}\right)$ is generated by $\left\{X_{1}, \sigma, X_{3}, \ldots, X_{n}\right\}, C_{p}\left(X_{1}\right)$ is generated by $\left\{X_{1}^{2}, \sigma, X_{3}, \ldots, X_{n}\right\}$.

Proof of the Claim. Let $K=\left\{a_{1}, \ldots, a_{n}\right\}$. Let $x_{1}, \ldots, x_{n-1}$ be a system of consecutive simple paths in $D$, s.t. $X_{i}=H\left(x_{i}\right)\left(H\left(x_{i}\right)\right.$ is the half-twist corresponding to $x_{i} ; x_{i}$ connects $a_{i}$ with $\left.a_{i+1}\right)$. Let $\Gamma_{1}, \ldots, \Gamma_{n}$ be a free geometric base of $\pi_{1}(D-K, *)$ consistent with $\left(X_{1}, \ldots, X_{n-1}\right)$ (that is, $\left(\Gamma_{i+1}\right) X_{i}=\Gamma_{i},\left(\Gamma_{i}\right) X_{i}=\Gamma_{i} \Gamma_{i+1} \Gamma_{i}^{-1},\left(\Gamma_{j}\right) X_{i}=\Gamma_{j}$ for $\left.j \neq i, i+1\right)$. We can assume that the $x_{i}$ does not intersect the "tails" of $\Gamma_{1}, \ldots, \Gamma_{n}$.

Let $K_{1}$ be a finite set of $D$ obtained from $K \cup\left\{x_{1}\right\}$ by contracting $x_{1}$ to a point $\tilde{a}_{2} \in x_{1} . K_{1}=\left\{\tilde{a}_{2}, a_{3}, \ldots, a_{n}\right\}$. Let $B_{n-1}=B_{n-1}\left[D, K_{1}\right]$. Let $Y_{2}, \ldots, Y_{n-1}$ be a frame of $B_{n-1}$ where $Y_{i}$ can be identified with $X_{i}$ for $i \geq 3$.

Let $H=\left\{b \in B_{n-1} \mid\left(\tilde{a}_{2}\right) b=\tilde{a}_{2}\right\}$. From the short exact sequence $1 \rightarrow P_{n-1} \hookrightarrow B_{n-1} \rightarrow S_{n-1} \rightarrow 1$ (see [MoTe4]) we can conclude that $H$ is generated by $Y_{3}, \ldots, Y_{n-1}$ and by the generators of $P_{n-1}$. We remove the generators of $P_{n-1}$ that can be expressed in terms of $Y_{3}, \ldots, Y_{n-1}$ (see [A],[B], and [MoTe4], Section IV) and conclude that $H$ is generated by $Y_{2}^{2}, Y_{3}, \ldots, Y_{n}$. The element $Y_{2}^{2}$ corresponds to the motion $\mathcal{M}^{\prime}$ of $\tilde{a}_{2}, a_{3}, \ldots, a_{n}$ described as follows: $\tilde{a}_{2}, a_{4}, \ldots, a_{n}$ stays in place and $a_{3}$ is moving around $\tilde{a}_{2}$ in the positive direction (see Fig. III.2.(a)).

We define a homomorphism $\Phi: C_{p}\left(X_{1}\right) \rightarrow H$ as follows:

Let $U$ be a "narrow" neighborhood of $x_{1}$ such that $\lambda=\partial U$ is a simple loop. Take $b \in C_{p}\left(X_{1}\right)$. There exists a representing diffeomorphism $\beta: D \rightarrow$ $D\left(\beta(K)=K,\left.\beta\right|_{\partial D}=\operatorname{Id}_{\partial D}\right)$ s.t. $\left.\beta\right|_{\bar{U}}=\operatorname{Id}_{\bar{U}}(\bar{U}=U \cup \lambda)$.

The diffeomorphism $\beta$ also defines an element of $B_{n-1}\left[D, K_{1}\right]$. This element is in fact in $H$ since $\tilde{a}_{2} \in x_{1}$ and thus $\left(\tilde{a}_{2}\right) \beta=\tilde{a}_{2}$. Denote this element by $\Phi(b)$. The map $\Phi$ constructed in this way is obviously a homomorphism, $\Phi: C_{p}\left(x_{1}\right) \rightarrow H$. Clearly, $X_{3}, \ldots, X_{n-1} \in C_{p}\left(X_{1}\right)$. Clearly, $\Phi\left(X_{i}\right)=Y_{i}$ for $i \geq 3$. Let $\mathcal{M}$ be the following motion in $(D, K): a_{1}, a_{2}, a_{4}, \ldots, a_{n}$ are stationary and $a_{3}$ goes around $a_{1}, a_{2}$ in the positive direction (Fig. III.2(b)). Let $u$ be the braid in $C_{p}\left(X_{1}\right)$ induced from the motion in $\mathcal{M}$. 


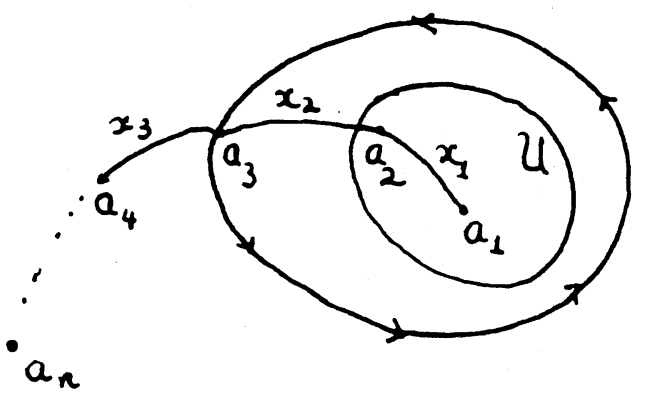

(b)

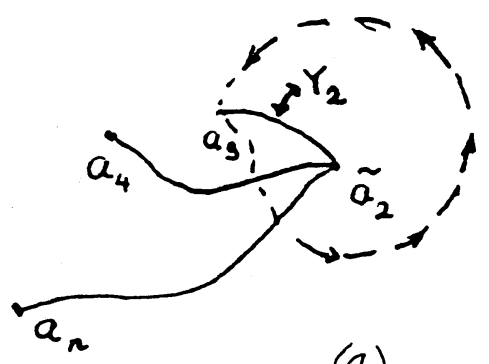

(a)

Figure III.2.

Clearly, $\Phi(u)=Y_{2}^{2}$. Thus, $\Phi$ is onto and $\Phi(u), \Phi\left(X_{3}\right), \ldots, \Phi\left(X_{n-1}\right)$ generate $h$. One can check that $u=Z_{31}^{2} Z_{32}^{2}$. But $Z_{31}=X_{2} X_{1} A_{1}^{-1}$ and $Z_{32}=X_{2}$. Thus $u=\sigma$. Thus $C_{p}\left(X_{1}\right)$ is generated by $\sigma, X_{3}, \ldots, X_{n-1}$ and a set of generators for $\operatorname{ker} \Phi$.

Consider $\pi_{1}\left(D-K \cup x_{1}, *\right)$. Let $\tilde{\Gamma}_{2}$ be the path obtained from connecting $\lambda$ with $* \in \partial D$ by a simple path intersecting each of $\Gamma_{3}, \ldots, \Gamma_{n}$ only at $*$. We get a (free) geometric base $\tilde{\Gamma}_{2}, \Gamma_{3}, \ldots, \Gamma_{n}$ of $\pi_{1}\left(D-\left(K \cup x_{1}\right), *\right)$. It is obvious that $\tilde{\Gamma}_{2}=\Gamma_{1} \Gamma_{2} . \Phi(b)$ defines in a natural way an automorphism of $\pi\left(D_{k} \cup\left\{X_{1}\right\}, *\right)$ s.t. $\Phi(b)$ does not change the product $\tilde{\Gamma}_{2} \Gamma_{3} \ldots \Gamma_{n}$, and $\left(\tilde{\Gamma}_{2}\right) \Phi(b)$ is a conjugate of $\tilde{\Gamma}_{2}$.

Consider now any $Z \in \operatorname{ker} \Phi$. We have $\left(\tilde{\Gamma}_{2}\right) Z=\tilde{\Gamma}_{2}\left(\tilde{\Gamma}_{2}=\Gamma_{1} \Gamma_{2}\right)$, $\left(\Gamma_{j}\right) Z=\Gamma_{j} \forall j=3, \ldots, n$. This implies that $Z$ can be represented by a diffeomorphism which is the identity outside of $U$, that is, $Z=X_{1}^{l}, l \in \mathbb{Z}$. Since $Z \in C_{p}\left(X_{1}\right)$, we get $l \equiv 0(\bmod 2)$.

Thus, $C_{p}\left(X_{1}\right)$ is generated by $X_{1}^{2}, \sigma, X_{3}, \ldots, X_{n-1}$. Clearly, $C\left(X_{1}\right)$ is generated by $C_{p}\left(X_{1}\right)$ and $X_{1}$.

the Claim

Lemma III.2.4. Let $\left\{\tilde{X}_{1}, \ldots, X_{n-1}\right\}$ be a frame in $B_{n}$, $\left(\tilde{X}_{1}, \ldots, \tilde{X}_{n-1}\right)$ their images in $\tilde{B}_{n}$. Let $u \in G$ (G is a $\tilde{B}_{n}$-group) be such that $u_{\tilde{X}_{1}^{-1}}=u^{-1} \tau$ with $\tau^{2}=1, \tau \in \operatorname{Center}(G), \tau_{b}=\tau \forall b \in \tilde{B}_{n} ;$

(2a) $u_{\tilde{X}_{2}^{-1} \tilde{X}_{1}^{-1}}=u^{-1} u_{\tilde{X}_{2}^{-1}}$;

(2b) $u_{\tilde{X}_{1} \tilde{X}_{2}^{-1} \tilde{X}_{1}^{-1}}=u_{\tilde{X}_{1}}^{-1} u_{\tilde{X}_{1} \tilde{X}_{2}^{-1}}$;

(3) $u_{\tilde{X}_{j}}=u \forall j=3, \ldots, n-1$. 
Then $u$ is a prime element in $G$, and $\tilde{X}_{1}$ is a supporting half-twist for $u$.

Proof. Let $Z \in B_{n}$ be any half-twist disjoint from $X_{1}, \tilde{Z}$ be the image of $Z$ in $\tilde{B}_{n} . \exists b \in B_{n}$ such that $\left(X_{1}\right)_{b}=X_{1},\left(X_{3}\right)_{b}=Z$. By Claim III.2.3, $b$ belongs to the subgroup of $B_{n}$ generated by $X_{1}, X_{3}, \ldots, X_{n-1}$ and $\sigma=X_{2} X_{1}^{2} X_{2}$. Let $\tilde{b}$ and $\tilde{\sigma}$ be the images of $b$ and $\sigma$ in $\tilde{B}_{n}$. We have $u_{\tilde{\sigma}^{-1}}=u_{\tilde{X}_{2}^{-1}} \tilde{X}_{1}^{-2} \tilde{X}_{2}^{-1}=$ $\left(u_{\tilde{X}_{2}^{-1}} \tilde{X}_{1}^{-1}\right)_{\tilde{X}_{1}^{-1} \tilde{X}_{2}^{-1}}=\left(u^{-1} u_{\tilde{X}_{2}^{-1}}\right)_{\tilde{X}_{1}^{-1} \tilde{X}_{2}^{-1}}=(\tau u)_{\tilde{X}_{2}^{-1}} \cdot u_{\tilde{X}_{2}^{-1} \tilde{X}_{1}^{-1} \tilde{X}_{2}^{-1}}=\tau u_{\tilde{X}_{2}^{-1}} \cdot$

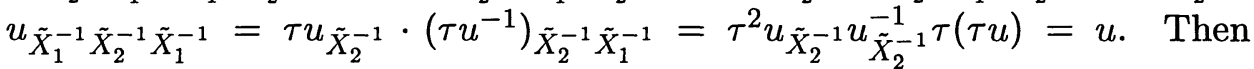
$u_{\tilde{\sigma}}=u$. Now: $u_{\tilde{X}_{j}}=u$ for $j \geq 3$ (by assumption (3)) and $u_{\tilde{X}_{1}^{2}}=u$ (by assumption (1)). Thus, if $X_{1}$ appears in $b$ an even number of times, then $u_{b}=u$. Otherwise we replace $b$ by $b X_{1}$. The "new" $b$ satisfies the same requirement for $b$, as above and $u_{\tilde{b}}=u$. Thus, we can assume $u_{\tilde{b}}=u$. We have

$$
u_{\tilde{Z}}=u_{\tilde{b}^{-1} \tilde{X}_{3} \tilde{b}}=u_{\tilde{X}_{3} \tilde{b}}=u_{\tilde{b}}=u .
$$

Let $Y$ be a half-twist in $B_{n}$ adjacent to $X_{1} . \exists b_{1} \in B_{n}$ s.t. $\left(X_{1}\right)_{b_{1}}=X_{1}$, $\left(X_{2}\right)_{b_{1}}=Y$. Let $\tilde{b}_{1}$ and $\tilde{Y}$ be the images of $b_{1}$ and $Y$ in $\tilde{B}_{n}$. As above, we can choose $b_{1}$ so that $u_{\tilde{b}_{1}}=u$. Applying $\tilde{b}_{1}$ on the assumptions $\left(2_{a}\right)$ and $\left(2_{b}\right)$ we get $\left(\right.$ since $\left.u_{\tilde{b}_{1}}=u,\left(\tilde{X}_{1}\right)_{\tilde{b}_{1}}=\tilde{X}_{1},\left(\tilde{X}_{2}\right)_{\tilde{b}_{1}}=\tilde{Y}\right)$ :

$$
u_{\tilde{Y}^{-1} \tilde{X}_{1}^{-1}}=u^{-1} u_{\tilde{Y}^{-1}} \text { and } u_{\tilde{X}_{1} \tilde{Y}^{-1} \tilde{X}_{1}^{-1}}=u_{\tilde{X}_{1}}^{-1} u_{\tilde{X}_{1} \tilde{Y}^{-1}} .
$$

\section{III.3. Polarized pairs and uniqueness of coherent pairs.}

Definition. Polarized pair

Let $G$ be a $\tilde{B}_{n}$-group, $h$ a prime element of $G, X$ its supporting halftwist. If $X$ is polarized, we say that $(h, X)$ (or $(h, \tilde{X})$ is a polarized pair with central element $\tau, \tau=h h_{\tilde{X}^{-1}}$.

Definition. Coherent pairs, Anti-coherent pairs

We say that two polarized pairs $\left(h_{1}, \tilde{X}_{1}\right)$ and $\left(h_{2}, \tilde{X}_{2}\right)$ are coherent (anticoherent) if $\exists \tilde{b} \in \tilde{B}_{n}$ such that $\left(h_{1}\right)_{\tilde{b}}=h_{2},\left(\tilde{X}_{1}\right)_{b}=\tilde{X}_{2}$, and $b$ preserves (reverses) the polarization.

Corollary III.3.1. Coherent and anti-coherent polarized pairs have the same central element. 
Proof. The prime elements of coherent and anti-coherent pairs are conjugate to each other. Thus by Lemma III.2.2 we get the corollary.

We need the following Lemma to prove later the unique existence of a prime element with given s.h.t. conjugate to a given prime element.

Lemma III.3.2. Let $h \in G$, a prime element with s.h.t. $\tilde{X}$. Let $b \in B_{n}$. Then: $\tilde{X}_{\tilde{b}}=\tilde{X} \Rightarrow h_{\tilde{b}}=h$.

Proof. We can choose a set of standard generators for $B_{n}[D, K],\left\{X_{1}, \ldots\right.$, $\left.X_{n-1}\right\}$ with $X_{1}=X$. Let $\sigma=X_{2} X_{1}^{2} X_{2}$. Consider $C_{p}\left(X_{1}\right)$, the centralizer of $X_{1}$ preserving polarization. By Lemma III.2.3, $C_{p}\left(X_{1}\right)$ is the subgroup of $B_{n}$ generated by $X_{1}^{2}, \sigma, X_{3}, \ldots, X_{n-1}$. Since $\tilde{X}_{3}, \ldots, \tilde{X}_{n-1}$ are disjoint from $X_{1}$, they do not change $h$ (by axiom(3) of prime elements). By Lemma

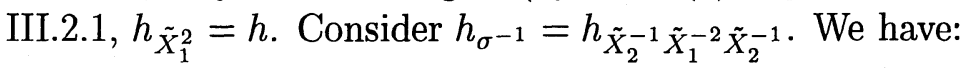

$$
\begin{aligned}
& h_{\tilde{\sigma}^{-1}}=h_{\tilde{X}_{2}^{-1} \tilde{X}_{1}^{-2} \tilde{X}_{2}^{-1}}=\left(h_{\tilde{X}_{2}^{-1}} \tilde{X}_{1}^{-1}\right)_{\tilde{X}_{1}^{-1}} \tilde{X}_{2}^{-1} \\
& \text { by Axiom(2) of prime element }\left(h^{-1} h_{\tilde{X}_{2}^{-1}}\right)_{\tilde{X}_{1}^{-1} \tilde{X}_{2}^{-1}} \\
& =\left(h_{\tilde{X}_{1}^{-1}}^{-1} h_{\tilde{X}_{2}^{-1} \tilde{X}_{1}^{-1}}\right)_{\tilde{X}_{2}^{-1}} \\
& \text { by Axiom(2) of prime element }\left(\tau h \cdot h^{-1} h_{\tilde{X}_{2}^{-1}}\right)_{\tilde{X}_{2}^{-1}}=\tau h_{\tilde{X}_{2}^{-2}} \\
& \text { by Lemma III.2.1(2) } \tau h \tau=h \text {. }
\end{aligned}
$$

Thus $h_{\tilde{\sigma}}=h$. Thus, for every generator $g$ of $C_{p}(X), h_{\tilde{g}}=h$. Since $b \in \tilde{C}(X)$, $h_{\tilde{B}}=h$.

Proposition III.3.3. Let $\{h, \tilde{X}\}$ be a polarized pair, $h \in G, \tilde{X} \in \tilde{B}_{n}$. Let $\tilde{T}$ be a polarized half-twist in $\tilde{B}_{n}$. Then there exists a unique prime element $g \in G$ such that $\{g, \tilde{T}\}$ and $\{h, \tilde{X}\}$ are coherent.

Proof. Let $X, T \in B_{n}$ be polarized half-twists representing $\tilde{X}$ and $\tilde{T}$. $\exists b \in$ $B_{n}$ such that $T=X_{b}$ preserving polarization. Let $\tilde{b}$ be the image of $b$ in $\tilde{B}_{n}$. Taking $g=h_{\tilde{b}}$ we obtain a polarized pair $\{g, \tilde{T}\}$ such that $\{g, \tilde{T}\}$ and $\{h, \tilde{X}\}$ are coherent. To prove the uniqueness of $g$, assume that $\left\{g_{1}, \tilde{T}\right\}$ is another polarized pair coherent with $\{h, \tilde{X}\}$. Then $\exists b_{1} \in B_{n}$ with $g=h_{\tilde{b}_{1}}$ and $T=X_{b_{1}}$, preserving polarization. We have $T=X_{b_{1}}=X_{b}$ and $X_{b_{1} b^{-1}}=X$. 
Denote $b_{2}=b_{1} b^{-1}$, so $X_{b_{2}}=X$ (preserving polarization). By the previous lemma, $h_{b_{2}}=h$. Thus, $h_{b_{1}}=h_{b}$ or $g=g_{1}$.

Definition. $L_{h, \tilde{X}}(\tilde{T})$

Let $(h, \tilde{X})$ be a polarized pair. $\tilde{T} \in \tilde{B}_{n} . L_{(h, \tilde{X})}(\tilde{T})$ is the unique prime element s.t. $\left(L_{(h, \tilde{X})}(\tilde{T}), \tilde{T}\right)$ is coherent with $(h, \tilde{X})$.

From uniqueness we get a simultaneous conjugation:

Lemma III.3.4. Assume $(h, \tilde{X})$ and $(g, \tilde{X})$ are polarized pairs. Let $\tau$ be the central element of $(g, \tilde{X})$. If $(h, \tilde{X})$ is anti-coherent to $(g, \tilde{X})$ then $h=g^{-1} \cdot \tau$.

Proof. By assumption, $\exists b \in B_{n}$ s.t. $g=h_{\tilde{b}}$ and $X=X_{b}$, reversing polarization. Thus $X_{b X^{-1}}=X$, preserving polarization. Thus $\left(h_{\tilde{b} \tilde{X}^{-1}}, \tilde{X}\right)$ is coherent with $\left(h_{\tilde{b} \tilde{X}^{-1}}, \tilde{X}_{\tilde{b} \tilde{X}^{-1}}\right)$. Clearly, $(h, \tilde{X})$ is coherent with $\left(h_{\tilde{b} \tilde{X}^{-1}}, \tilde{X}_{\tilde{b} \tilde{X}^{-1}}\right)$. From uniqueness, $h=h_{\tilde{b}_{\tilde{X}}^{-1}}=g_{\tilde{X}^{-1}}$. Since $\tau$ is the central element of $(g, \tilde{X})$, $\tau=g \cdot g_{\tilde{X}^{-1}}$. Thus, $g_{X^{-1}}=g^{-1} \tau$. So $h=g^{-1} \tau$.

Corollary III.3.5. If $\left(a_{i}, \tilde{X}\right)$ is coherent with $\left(g_{i}, \tilde{Y}\right) i=1,2$, then there exist $b \in \tilde{B}$ s.t. $(a)_{b}=g_{i} i=1,2$.

Proof. Let $b$ be the element of $\tilde{B}_{n}$ s.t. $\left(a_{1}\right)_{b}=g_{1},(\tilde{X})_{b}=\tilde{Y}$. Now, $\left(\left(a_{2}\right)_{b},(X)_{b}\right)$ is coherent with $\left(a_{2}, \tilde{X}\right)$. Since $(\tilde{X})_{b}=\tilde{Y},\left(\left(a_{2}\right)_{b}, \tilde{Y}\right)$ is coherent with $\left(a_{2}, \tilde{X}\right)$. The pair $\left(g_{2}, \tilde{Y}\right)$ is also coherent with $\left(a_{2}, \tilde{X}\right)$. From uniqueness, $\left(a_{2}\right)_{b}=g_{2}$.

\section{III.4. $\tilde{\mathbf{B}}_{\mathbf{n}}$-action of nondisjoint half-twists.}

Proposition III.4.1. Let $\tilde{T}, \tilde{Y}$ be 2 orderly adjacent polarized half-twists in $\tilde{B}_{n},\{h, \tilde{X}\}$ be a polarized pair, $h \in G, \tilde{X} \in \tilde{B}_{n}$. Denote by $\tilde{Y}^{\prime}$ the polarized half-twist obtained from $\tilde{Y}$ by changing polarization (that is, $\tilde{T}, \tilde{Y}^{\prime}$ are not orderly adjacent). Denote by

$$
\begin{aligned}
L(T) & =L_{\{h, \tilde{X}\}}(\tilde{T}), \\
L(Y) & =L_{\{h, \tilde{X}\}}(\tilde{Y}), \\
L\left(Y^{\prime}\right) & =L_{\{h, \tilde{X}\}}\left(\tilde{Y}^{\prime}\right) .
\end{aligned}
$$


Then

(1) $L(T)_{\tilde{T}^{-1}}=L(T)^{-1} \tau$

(2) $L(T)_{\tilde{Y}^{-1}}=L(T) L(Y)$;

(3) $L(T)_{\left(\tilde{Y}^{\prime}\right)^{-1}}=L(Y)^{-1} L(T)$.

Proof.

(1) By Lemma III.2.1(1).

(2) Let $b \in B_{n}$ be s.t. $L(T)=h_{b}, T=X_{b}$, preserving polarization. Let $Y_{1}=Y_{b^{-1}}$. Then $\left\{X, Y_{1}\right\}$ is a pair of adjacent half-twists $\left(X=T_{b^{-1}}, Y=\right.$ $\left.Y_{b^{-1}}\right)$, and so $h_{\tilde{Y}_{1}^{-1} \tilde{X}^{-1}}=h^{-1} h_{\tilde{Y}_{1}^{-1}}$. Applying $\tilde{b}$ to that equation, we get $(L(T))_{\tilde{Y}^{-1} \tilde{T}^{-1}}=L(T)^{-1} L(T)_{\tilde{Y}^{-1}}$, or

$$
L(T)_{\tilde{Y}^{-1}}=L(T) \cdot L(T)_{\tilde{Y}^{-1} \tilde{T}^{-1}}
$$

Let $b_{1}=b Y^{-1} T^{-1}$. Then $X_{b_{1}}=X_{b Y^{-1} T^{-1}}=T_{Y^{-1} T^{-1}}=Y$ (since $T_{Y^{-1}}=Y_{T}$ by III.0). Using that $T, Y$ are orderly adjacent and $X_{b}=T$, preserving polarization, one can easily check that actually $X_{b_{1}}=Y$, preserving polarization. Because of the uniqueness of $L(Y)=L_{\{h, \tilde{X}\}}(\tilde{Y})$, we get $L(Y)=h_{\tilde{b}_{1}}=h_{\tilde{b} \tilde{Y}^{-1} \tilde{T}^{-1}}=L(T)_{\tilde{Y}^{-1} \tilde{T}^{-1}}$. Together with the previous equation we get: $L(T)_{\tilde{Y}^{-1}}=L(T) L(Y)$, which is (2).

(3) Using $Y_{Y^{-1}}=Y^{\prime}$ (preserving polarization) and uniqueness, we can write $L\left(Y^{\prime}\right)=L(Y)_{\tilde{Y}^{-1}}$. By $(1), L(Y)_{\tilde{Y}^{-1}}=L(Y)^{-1} \tau$. Thus, $L\left(Y^{\prime}\right)=$ $L(Y)^{-1} \tau$. From (2) we get: $L(T)_{\tilde{Y}^{\prime-1}}=L(T) L\left(Y^{\prime}\right)=L(T) L(Y)^{-1} \tau=$ $L(Y)^{-1} L(T)$, which is $(3)$.

(We used $\left[L(T), L(T)_{\tilde{Y}^{-1}}\right]=\tau$, from Lemma III.2.1, which implies $\tau=$ $[L(T), L(T) L(Y)]=[L(T), L(Y)]$. )

Lemma III.4.2. Let $h$ be a prime element in $G, \tilde{X} \in \tilde{B}_{n}$ a supporting half-twist of $h, \tilde{Z}$ a half-twist in $\tilde{B}_{n}$ transversal to $\tilde{X}$. Then $h_{\tilde{Z}}=h$.

Proof. Let $X, Z$ be transversal half-twists in $B_{n}$, representing $\tilde{X}, \tilde{Z}$. Let $x, z$ be 2 transversally intersecting simple paths corresponding to $X, Z$ (see Fig. III.4).

There exists a simple path $y$ such that the corresponding half-twist $Y$ is adjacent to $X$ and $Z$, and $Z_{1}=Z_{Y^{-2}}$ is disjoint from $X$. Let $z_{1}$ be the path corresponding to $Z_{1}$ (see Fig. III.4). Denote by $\tilde{Y}, \tilde{Z}_{1}$ the images of $Y, Z_{1}$ in 


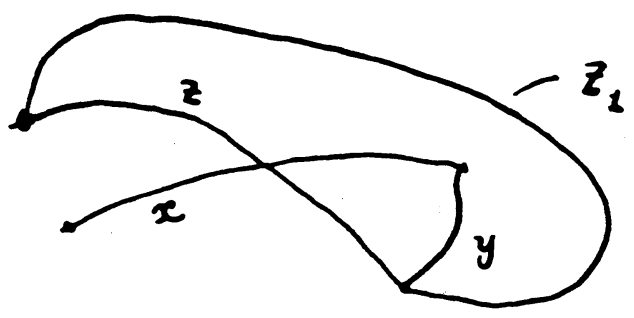

Figure III.4.

$\tilde{B}_{n}$. We have $h_{\tilde{Z}}=h_{\tilde{Y}^{-2} \tilde{Z}_{1} \tilde{Y}^{2}}$ by Lemma III.2.1 $(h \tau)_{\tilde{Z}_{1} \tilde{Y}^{2}}=(h \tau)_{\tilde{Y}^{2}}=h \tau \cdot \tau=h$.

\section{III.5. Commutativity properties.}

Proposition III.5.1. Let $\left\{g_{1}, \tilde{Y}_{1}\right\},\left\{g_{2}, \tilde{Y}_{2}\right\}$ be 2 polarized pairs in $G$. Assume that they are coherent or anti-coherent. Let $\tau$ be the corresponding central element of $\left(g_{1}, \tilde{Y}_{1}\right) \quad\left(\tau=g_{1}\left(g_{1}\right)_{\tilde{Y}_{1}^{-1}}\right)$.

Then

(1) if $\tilde{Y}_{1}, \tilde{Y}_{2}$ are adjacent, then $\left[g_{1}, g_{2}\right]=\tau$;

(2) if $\tilde{Y}_{1}, \tilde{Y}_{2}$ are disjoint or transversal, then $\left[g_{1}, g_{2}\right]=1$.

Proof.

(1) Assume first that $\left\{g_{1}, \tilde{Y}_{1}\right\},\left\{g_{2}, \tilde{Y}_{2}\right\}$ are coherent. Take $b \in \tilde{B}_{n}$ with $g_{2}=\left(g_{1}\right)_{b}, \tilde{Y}_{2}=\left(\tilde{Y}_{1}\right)_{b}$ (preserving polarization). Let $b_{1}=\tilde{Y}_{2}^{-1} \tilde{Y}_{1}^{-1}$. Then $\left(\tilde{Y}_{1}\right)_{b_{1}}=\tilde{Y}_{2}$. Assume that $b_{1}$ preserves polarization of $\tilde{Y}_{1}, \tilde{Y}_{2}$. We have $\left\{\left(g_{1}\right)_{b_{1}}, \tilde{Y}_{2}\right\}$ and $\left\{g_{2}, \tilde{Y}_{2}\right\}$ coherent with $\left\{g_{1}, \tilde{Y}_{1}\right\}$. By Proposition III.3.3 (the uniqueness part) we get $\left(g_{1}\right)_{b_{1}}=g_{2}$. Thus we have $g_{2}=\left(g_{1}\right)_{b_{1}}=\left(g_{1}\right)_{\tilde{Y}_{2}^{-1} \tilde{Y}_{1}^{-1}}=g_{1}^{-1}\left(g_{1}\right)_{\tilde{Y}_{2}^{-1}}$, and $\left[g_{1}, g_{2}\right]=\left[g_{1}, g_{1}^{-1}\left(g_{1}\right)_{\tilde{Y}_{2}^{-1}}\right]=$ $g_{1} g_{1}^{-1}\left(g_{1}\right)_{\tilde{Y}_{2}^{-1}} g_{1}^{-1}\left(g_{1}^{-1}\right)_{\tilde{Y}_{2}^{-1}} g_{1}=\left[g_{1},\left(g_{1}\right)_{\tilde{Y}_{2}^{-1}}\right]_{g_{1}} \stackrel{\text { by Lemma III.2.1(3) }}{=} \tau_{g_{1}}=\tau$. 
If $b_{1}$ does not preserve the polarization of $\tilde{Y}_{1}, \tilde{Y}_{2}$, consider $b_{2}=b_{1} \tilde{Y}_{2}$. Then $\tilde{Y}_{2}=\left(\tilde{Y}_{1}\right)_{b_{2}}$, preserving polarization. As above, we get

$$
\begin{aligned}
& g_{2}=\left(g_{1}\right)_{b_{2}}
\end{aligned}
$$

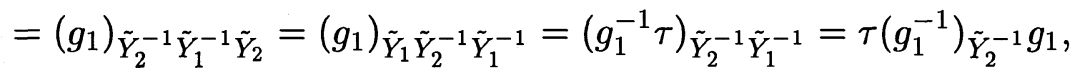

and then

$$
\begin{aligned}
{\left[g_{1}, g_{2}\right]=\left[g_{1}, \tau\right.} & \left.\left(g_{1}^{-1}\right)_{\tilde{Y}_{2}^{-1}} g_{1}\right] \\
& =\left[g_{1},\left(g_{1}^{-1}\right)_{\tilde{Y}_{2}^{-1}}\right]=\left[g_{1},\left(g_{1}\right)_{\tilde{Y}_{2}^{-1}}\right]_{\left(g_{1}\right)_{\tilde{Y}_{2}^{-1}}^{-1}} \stackrel{\text { by Lemma III.2.1(3) }}{=} \tau .
\end{aligned}
$$

If $\left\{g_{1}, \tilde{Y}_{1}\right\},\left\{g_{2}, \tilde{Y}_{2}\right\}$ are anti-coherent, denote $\tilde{Y}_{2}^{\prime}$ the half-twist obtained from $\tilde{Y}_{2}$ by changing polarization. One can then check that $\left\{g_{1}, \tilde{Y}_{1}\right\}$, $\left\{g_{2 ; \tilde{Y}_{2}^{-1}}, \tilde{Y}_{2}^{\prime}\right\}$ are coherent. We have from the above that $\tau=\left[g_{1},\left(g_{2}\right)_{\tilde{Y}_{2}^{-1}}\right]$. By Corollary III.3.1 $\tau$ is also the central element of $\left(g_{2}, \tilde{Y}_{2}\right)$. Thus $\tau=g_{2}\left(g_{2}\right)_{\tilde{Y}_{2}^{-1}}$ which implies $\left(g_{2}\right)_{\tilde{Y}_{2}^{-1}}=g_{2}^{-1} \tau$. Thus $\tau=\left[g_{1},\left(g_{2}\right)_{\tilde{Y}_{2}^{-1}}\right]=\left[g_{1}, g_{2}^{-1} \tau\right]=$ $\left[g_{1}, g_{2}^{-1}\right]=\left[g_{1}, g_{2}\right]_{g_{2}}^{-1}$. Thus, $\left[g_{1}, g_{2}\right]=\tau_{g_{2}}^{-1}=\tau$.

(2) We can assume that $\left\{g_{1}, \tilde{Y}_{1}\right\},\left\{g_{2}, \tilde{Y}_{2}\right\}$ are coherent. (Otherwise, we replace $\tilde{Y}_{2}$ by $\tilde{Y}_{2}^{\prime}$ and $g_{2}$ by $\left(g_{2}\right)_{\tilde{Y}_{2}^{-1}}$ and use $\left[g_{1},\left(g_{2}\right) \tilde{Y}_{2}^{-1}\right]=\left[g_{1}, g_{2}\right]_{g_{2}}^{-1}$ (see above).)

Consider first the case where $\tilde{Y}_{1}, \tilde{Y}_{2}$ are disjoint. We can choose a standard base of $\tilde{B}_{n}$, say $\left(\tilde{X}_{1}, \tilde{X}_{2}, \ldots, \tilde{X}_{n-1}\right)$ such that $\tilde{X}_{1}=\tilde{Y}_{1}, \tilde{X}_{3}=\tilde{Y}_{2}$ and the given polarizations of $\tilde{Y}_{1}, \tilde{Y}_{2}$ coincide with "consecutive" polarizations of $\tilde{X}_{1}, \tilde{X}_{3}$ ("end" of $\tilde{X}_{1}=$ "origin" of $\tilde{X}_{2}$, "end" of $\tilde{X}_{2}=$ "origin" of $\tilde{X}_{3}$ ). Let $b_{1}=\tilde{X}_{2}^{-1} \tilde{X}_{1}^{-1} \tilde{X}_{3}^{-1} \tilde{X}_{2}^{-1}$. Then $\tilde{Y}_{2}=\left(\tilde{Y}_{1}\right)_{b_{1}}$, preserving polarization. From Proposition III.3.3 (uniqueness) it follows that

$$
\begin{aligned}
& g_{2}=\left(g_{1}\right)_{b_{1}} \\
& =\left(g_{1}\right)_{\tilde{X}_{2}^{-1}} \tilde{X}_{1}^{-1} \tilde{X}_{3}^{-1} \tilde{X}_{2}^{-1}=\left(g_{1}^{-1}\left(g_{1}\right)_{\tilde{X}_{2}^{-1}}\right)_{\tilde{X}_{3}^{-1} \tilde{X}_{2}^{-1}}=\left(g_{1}^{-1}\right)_{\tilde{X}_{2}^{-1}}\left(g_{1}\right)_{\tilde{X}_{2}^{-1}} \tilde{X}_{3}^{-1} \tilde{X}_{2}^{-1}
\end{aligned}
$$

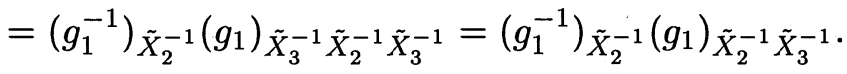

By III.2.1(3) $\left[g_{1},\left(g_{1}\right)_{\tilde{X}_{2}^{-1}}\right]=\tau$, which implies

$$
\left[g_{1},\left(g_{1}\right)_{\tilde{X}_{2}^{-1} \tilde{X}_{3}^{-1}}\right]=\left[g_{1},\left(g_{1}\right)_{\tilde{X}_{2}^{-1}}\right]_{\tilde{X}_{3}^{-1}}=\tau_{\tilde{X}_{3}^{-1}}=\tau .
$$

We can write

$$
\begin{aligned}
{\left[g_{1}, g_{2}\right]=\left[g_{1},\right.} & \left(g_{1}^{-1}\right)_{\tilde{X}_{2}^{-1}}\left(g_{1}\right)_{\left.\tilde{X}_{2}^{-1} \tilde{X}_{3}^{-1}\right]} \\
& =\left[g_{1},\left(g_{1}^{-1}\right)_{\tilde{X}_{2}^{-1}}\right] \cdot\left[g_{1},\left(g_{1}\right)_{\tilde{X}_{2}^{-1} \tilde{X}_{3}^{-1}}\right]_{\left(g_{1}\right)_{\tilde{X}_{2}^{-1}}}=\tau \cdot \tau=\tau^{2}=1
\end{aligned}
$$


Assume now that $\tilde{Y}_{1}, \tilde{Y}_{2}$ are transversal. As in the proof of Lemma III.4.2, we can find a half-twist $\tilde{T} \in \tilde{B}_{n}$ such that $\tilde{T}$ is adjacent to $\tilde{Y}_{1}, \tilde{Y}_{2}$ and $\tilde{Y}_{2}^{\prime}=\left(\tilde{Y}_{2}\right)_{\tilde{T}^{-2}}$ is disjoint from $\tilde{Y}_{1}$. Let $b \in \tilde{B}_{n}$ be such that $\tilde{Y}_{2}=\left(\tilde{Y}_{1}\right)_{b}$, $g_{2}=\left(g_{1}\right)_{b}$. Let $b^{\prime}=b \tilde{T}^{-2}, g_{2}^{\prime}=\left(g_{1}\right)_{b^{\prime}}=\left(g_{2}\right)_{\tilde{T}_{2}^{-2}}$. Then $\left\{g_{2}^{\prime}, \tilde{Y}_{2}^{\prime}\right\}$ is coherent, or anti-coherent, with $\left\{g_{1}, \tilde{Y}_{1}\right\}$. Since $\tilde{Y}_{2}^{\prime}, \tilde{Y}_{1}$ are disjoint, we get from the above $\left[g_{1}, g_{2}^{\prime}\right]=1$. By Lemma III.2.1 $g_{2}^{\prime}=\left(g_{2}\right)_{\tilde{T}^{-2}}=g_{2} \tau$, or $g_{2}=g_{2}^{\prime} \tau$. Therefore, $\left[g_{1}, g_{2}\right]=\left[g_{1}, g_{2}^{\prime} \tau\right]=\left[g_{1}, g_{2}^{\prime}\right]=1$.

Recall that here exists a natural homomorphism $\psi_{n}: B_{n} \rightarrow S_{n} . \psi_{n}\left(X_{i}\right)$ is the transposition $(i i+1)$ for $X_{i}$ a half-twist connecting the points $q_{i}$ and $q_{i+1}$.

Definition. $P_{n}$

$P_{n}=\operatorname{ker} \psi_{n}$.

Recall from [MoTe4] that: $P_{n}$ is generated by $Z_{i j}^{2}$, where:

$$
Z_{i j}=\left(X_{i}^{2}\right)_{X_{i+1} \ldots X_{j-1}} \text {. }
$$

Definition. $\tilde{P}_{n}$

$$
\tilde{P}_{n}=\operatorname{ker}\left(\tilde{B}_{n} \stackrel{\tilde{\psi}_{n}}{\rightarrow} S_{n}\right) \text { where } \psi_{n} \text { is induced naturally from } \psi_{n}
$$

Proposition III.5.2. Assume $n \geq 4$. Let $\tilde{X}_{1}, \tilde{X}_{2}$ be 2 adjacent half-twists in $\tilde{B}_{n}$. Let $c=\left[\tilde{X}_{1}^{2}, \tilde{X}_{2}^{2}\right]$. Then the commutant $\tilde{P}_{n}^{\prime}$ of $\tilde{P}_{n}$ is generated by $c$ where $c_{b}=c \forall b \in \tilde{B}_{n}$, and $c^{2}=1$. Moreover, if $\left(\tilde{Y}_{1}, \tilde{Y}_{2}\right)$ and $\left(\tilde{Z}_{1}, \tilde{Z}_{2}\right)$ are two pairs of adjacent half-twists, then $\left[\tilde{Z}_{1}^{2}, \tilde{Z}_{2}^{2}\right]=\left[\tilde{Y}_{1}^{2}, \tilde{Y}_{2}^{2}\right]=\left[\tilde{Z}_{1}^{2}, \tilde{Z}_{2}^{-2}\right]=$ $\left[\tilde{Z}_{1}^{-2}, \tilde{Z}_{2}^{-2}\right]=c$.

Proof. Let $\left.B_{n}=B_{n}(D, K)\right)$. Complete $\tilde{X}_{1}$ and $\tilde{X}_{2}$ to $\tilde{X}_{1}, \ldots, \tilde{X}_{n-1}$, a standard base of $\tilde{B}_{n}, \quad X_{i}=\left(H\left(x_{i}\right)\right.$ and $x_{1}, \ldots, x_{n-1}$ are simple paths in $D$. Let $c=\left[\tilde{X}_{1}^{2}, \tilde{X}_{2}^{2}\right]$. Let $x=\left(x_{1}\right)_{\tilde{X}_{2} \tilde{X}_{3}}$. We have a quadrangle formed by $x_{1}, x_{2}, x_{3}, x$, (see Fig. III.5(a)).

Denote by $X \in B_{n}$ the half-twist defined by $x$. Evidently, $\tilde{X}_{1}, \tilde{X}_{2}, \tilde{X}_{3}, \tilde{X}$ form a good quadrangle in $\tilde{B}_{n}$. Thus by Lemma III.1.2

$$
\tilde{X}_{1}^{2} \tilde{X}_{3}^{2}=\tilde{X}_{2}^{2} \tilde{X}^{2} .
$$

Denote $y_{1}=\tilde{X}_{1}^{2}, \quad y_{2}=\tilde{X}_{2}^{2}, \quad y_{3}=\tilde{X}_{3}^{2}, \quad y_{4}=\tilde{X}^{2}$, (the squares of the edges), $d_{1}=\tilde{X}_{1} \tilde{X}_{2}^{2} \tilde{X}_{1}^{-1}, d_{2}=\tilde{X}_{2} \tilde{X}_{3}^{2} \tilde{X}_{2}^{-1}$, (the squares of the diagonals), $y^{\prime}=\tilde{X}_{2} \tilde{X}_{1}^{2} \tilde{X}_{2}^{-1}$ (the square of the outer diagonal) 


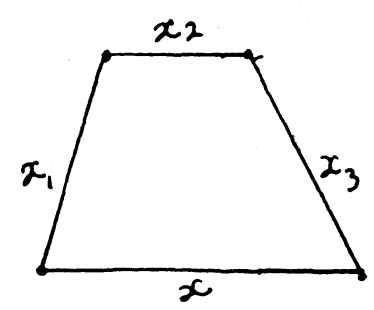

(a)

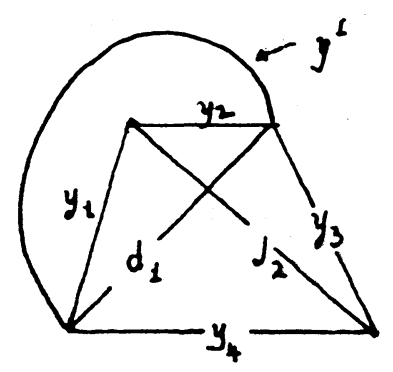

(b)

Figure III.5.

See Fig. III.5(b) where we denote the paths corresponding to the half-twists whose squares we considered here.

Clearly:

$y_{1} y_{3}=y_{3} y_{1}$

$d_{1}=\left(y^{\prime}\right)_{y_{1}^{-1}}$

$\left(y_{3}\right)_{x_{2}}=\left(d_{2}\right)_{x_{3}^{-2}}=\left(d_{2}\right)_{y_{3}^{-1}}$

$y_{1} y_{2} d_{1}=y_{2} y_{1} y^{\prime}=\Delta_{3}^{2}$ (a central element of $\left.P_{3}\right)$.

We rewrite (1.10) to get

$$
y_{4}=y_{1} y_{3} y_{2}^{-1}
$$

Conjugating (1.11) by $\tilde{X}_{1}$, we get:

$$
d_{2}=y_{1} y_{3} y^{\prime-1}
$$

conjugating (1.11) by $\tilde{X}_{2}$ we get $y_{4}=d_{1}\left(d_{2}\right)_{y_{3}^{-1}} \cdot y_{2}^{-1}$. Since $d_{1}=y_{1} y^{\prime} y_{1}^{-1}$,

$$
y_{4}=y_{1} y^{\prime} y_{1}^{-1} y_{3} d_{2} y_{3}^{-1} y_{2}^{-1} \stackrel{\text { by }}{\stackrel{(1.12)}{=}} y_{1} y^{\prime} y_{1}^{-1} y_{3} y_{1} y_{3} y^{\prime-1} y_{3}^{-1} y_{2}^{-1} \text {. }
$$

We compare the last expression with (1.11) to get:

$$
y^{\prime} y_{3}^{2} y^{\prime-1} y_{3}^{-1}=y_{3}, \quad \text { or } \quad\left[y^{\prime}, y_{3}^{2}\right]=1 .
$$

Since $y^{\prime}, y_{3}$ are squares of two adjacent half-twists in $\tilde{B}_{n}$, and any two pairs of adjacent half-twists are conjugate, we conclude from (1.13) that:

(1.14) $\forall$ pairs of adjacent half-twists, say $\tilde{Z}_{1}, \tilde{Z}_{2}$ in $\tilde{B}_{n}:\left[\tilde{Z}_{1}^{2}, \tilde{Z}_{2}^{4}\right]=1$, which also implies that $\left[\tilde{Z}_{1}^{2}, \tilde{Z}_{2}^{2}\right]=\left[\tilde{Z}_{1}^{-2}, \tilde{Z}_{2}^{2}\right]=\left[\tilde{Z}_{1}^{2}, \tilde{Z}_{2}^{-2}\right]=\left[\tilde{Z}_{1}^{-2}, \tilde{Z}_{2}^{-2}\right]$. 
Conjugating (1.11) by $\tilde{X}_{3}^{-1}$ we get:

$$
d_{1}=y_{1} y_{3}\left(d_{2}\right)_{y_{3}^{-1}}=y_{1} y_{3} \cdot y_{3} d_{2}^{-1} y_{3}^{-1}=y_{1} y_{3}^{2} \cdot d_{2}^{-1} y_{3}^{-1} \stackrel{(1.14)}{=} y_{1} d_{2}^{-1} y_{3}
$$

We have by $(1.14)$ that $1=\left[y_{1}^{2}, y_{2}\right]=\left[y_{1}, y_{2}\right]_{y_{1}^{-1}} \cdot\left[y_{1}, y_{2}\right]$. Denoting $c=\left[y_{1}, y_{2}\right]$, we can write:

$$
c_{y_{1}^{-1}}=c^{-1}, \quad \text { or } \quad c_{y_{1}}=c^{-1} \text {. }
$$

Denote by $\tilde{P}_{3}$ the subgroup of $\tilde{B}_{n}$ generated by $y_{1}, y_{2}, d_{1}$, and by $\alpha=\Delta_{3}^{2}=$ $y_{1} y_{2} d_{1}=y_{2} y_{1} y^{\prime}$ (a central element of $\tilde{P}_{3}$ ), so that

$$
y^{\prime}=y_{1}^{-1} y_{2}^{-1} \alpha
$$

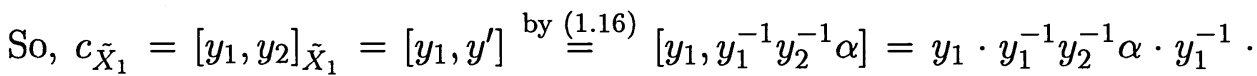
$\alpha^{-1} y_{2} y_{1}=y_{2}^{-1} y_{1}^{-1} y_{2} y_{1}=\left[y_{2}^{-1}, y_{1}^{-1}\right]=\left[y_{2}, y_{1}\right]=c^{-1}$. Thus we have $c_{\tilde{X}_{1}^{2}}=$ $\left(c^{-1}\right)_{\tilde{X}_{1}}=c$.

By $(1.15) c_{\tilde{X}_{1}^{2}}=c_{y_{1}}=c^{-1}$.

We compare the last two results to get $c=c^{-1}$ or

$$
c^{2}=1 \quad \text { and } \quad c_{\tilde{X}_{1}}=c .
$$

Using a conjugation which sends $\left(\tilde{X}_{1}, \tilde{X}_{2}\right)$ to $\left(\tilde{X}_{2}, \tilde{X}_{1}\right)$, we obtain from

$$
c_{\tilde{X}_{2}}^{-1}=c^{-1}, \quad \text { or } \quad c_{\tilde{X}_{2}}=c
$$

(1.17) and (1.18) show that $\forall z \in \tilde{B}_{3}$ (the subgroup of $\tilde{B}_{n}$ generated by $\left.\tilde{X}_{1}, \tilde{X}_{2}\right)$ we have

$$
c_{z}=c
$$

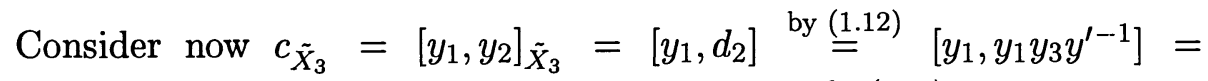
$y_{1} y_{1} y_{3} y^{\prime-1} y_{1}^{-1} y^{\prime} y_{3}^{-1} y_{1}^{-1}=y_{1} y_{3} \cdot\left(y_{1} y^{\prime-1} y_{1}^{-1} y^{\prime}\right) y_{3}^{-1} y_{1}^{-1} \stackrel{\text { by } \stackrel{(1.19)}{=}}{=} y_{1} y_{3} c y_{3}^{-1} y_{1}^{-1}=$ $c_{y_{1}^{-1} y_{3}^{-1}}=c_{y_{1}^{-3}}=c_{y_{3}}=c_{\tilde{X}_{3}^{2}}$, shortly $c_{\tilde{X}_{3}^{2}}=c_{\tilde{X}_{3}}$. This implies $c_{\tilde{X}_{3}}=c$.

Since $c=\left[\tilde{X}_{1}^{2}, \tilde{X}_{2}^{2}\right]$, we have $\forall \tilde{X}_{j}, j \geq 4, c_{\tilde{X}_{j}}=c$. Thus $\forall b \in \tilde{B}_{n} c_{b}=c$ and $c^{2}=1$.

Let $\left(\tilde{Y}_{1}, \tilde{Y}_{2}\right)$ be a pair of adjacent half-twists. Since every 2 pairs of adjacent half-twists are conjugate in $\tilde{B}_{n}, \quad \exists b \in \tilde{B}_{n}$ s.t. $\left[\tilde{Y}_{1}^{2}, \tilde{Y}_{2}^{2}\right]=\left[\tilde{X}_{1}, \tilde{X}_{2}^{2}\right]_{b}=$ 
$c_{b}$. Since $c_{b}=c \quad \forall b \in \tilde{B}_{n},\left[\tilde{Y}_{1}^{2}, \tilde{Y}_{2}^{2}\right]=c$. Since $c^{2}=1, c \in \operatorname{Center}\left(\tilde{B}_{n}\right)$ we also have $\left[\tilde{Y}_{1}^{2}, \tilde{Y}_{2}^{-2}\right]=\left[\tilde{Y}_{1}^{-2}, \tilde{Y}_{2}^{-2}\right]=c$. In particular, if $\left(\tilde{Z}_{1}, \tilde{Z}_{2}\right)$ is another pair of adjacent half-twists, $\left[\tilde{Z}_{1}^{2}, \tilde{Z}_{2}^{2}\right]=\left[\tilde{Y}_{1}^{2}, \tilde{Y}_{2}^{2}\right]=c$. Because any two disjoint and transversal half-twists of $\tilde{B}_{n}$ commute, and $\tilde{P}_{n}$ is generated by $\tilde{Z}_{i j}^{2}=\left(\tilde{X}_{i}^{2}\right)_{\tilde{X}_{i+1} \cdots \tilde{X}_{j-1}}, 1 \leq i<j \leq n$ (see [MoTe4]), we conclude that $\tilde{P}_{n}^{\prime}$ is generated by $c$.

\section{III.6. $\tilde{\mathbf{P}}_{\mathbf{n}}$ as a $\tilde{\mathbf{B}}_{\mathbf{n}}$-group.}

Recall: $\operatorname{Ab}\left(B_{n}\right) \simeq \mathbb{Z}\left(B_{n}\right.$ is generated by the half-twists and every 2 half-twists are conjugate).

Definition. $P_{n, 0}$

$P_{n, 0}=\operatorname{ker}\left(P_{n} \rightarrow \mathrm{Ab} B_{n}\right)$ ("degree zero" pure braids).

Definition. $\tilde{P}_{n, 0}$

$\tilde{P}_{n, 0}$ is the image of $P_{n, 0}$ in $\tilde{P}_{n}$.

Lemma III.6.1. Let $X_{1}, X_{2}$ be 2 consecutive half-twists in $B_{n}$. Let $u=$ $\left(\tilde{X}_{1}^{2}\right)_{\tilde{X}_{2}^{-1}} \tilde{X}_{2}^{-2}$. Then $u \in \tilde{P}_{n, 0} u$ is a prime element in $\tilde{P}_{n}$ (considered as a $\tilde{B}_{n}$-group), and $\tilde{X}_{1}$ is the supporting half-twist of $u$.

Proof. Clearly, $u \in \tilde{P}_{n, 0}$. Since $X_{1} X_{2} X_{1}=X_{2} X_{1} X_{2}, \quad\left(X_{1}\right)_{X_{2}^{-1}}=\left(X_{2}\right)_{X_{1}}$ and thus, $u=\left(\tilde{X}_{1}\right)_{\tilde{X}_{2}^{-1}}^{2} \tilde{X}_{2}^{-2}=\left(\tilde{X}_{2}\right)_{\tilde{X}_{1}}^{2} \tilde{X}_{2}^{-2}$. We often use here the fact that $\left(X_{1}\right)_{X_{2}^{-1}}=\left(X_{2}\right)_{X_{1}}$ as well as the fact that $\left[\tilde{X}_{1}^{ \pm 2}, X_{2}^{ \pm 2}\right]=c$, i.e., $\tilde{X}_{2}^{-2} \tilde{X}_{2}^{2}=$ $c \tilde{X}_{2}^{2} \tilde{X}_{1}^{-2}$ and $\left(\tilde{X}_{1}^{2}\right)_{X_{2}^{-1}}=\left(\tilde{X}_{1}^{2}\right)_{X_{2}} c$ for $c \in \operatorname{Center}\left(\tilde{B}_{n}\right), c^{2}=1$. Complete $X_{1}, X_{2}$ to a frame of $B_{n}: X_{1}, \ldots, X_{n-1} \cdot\left(\left\langle X_{i}, X_{i+1}\right\rangle=1\right.$ and $\left[X_{i}, X_{j}\right]=1$ $|i-j|>2$.). We shall use Lemma III.2.4, that is, we must check conditions (1), $\left(2_{a}\right),\left(2_{b}\right),(3)$ of Lemma III.2.4.

(1) We have $u_{\tilde{X}_{1}^{-1}}=\left(\tilde{X}_{2}^{2}\right)_{\tilde{X}_{1} \tilde{X}_{1}^{-1}} \cdot\left(\tilde{X}_{2}^{-2}\right)_{\tilde{X}_{1}^{-1}}=\tilde{X}_{2}^{2} \cdot\left(\tilde{X}_{1}^{-2}\right)_{\tilde{X}_{2}}$. Since $c=$ $\left[\tilde{X}_{1}^{2}, \tilde{X}_{2}^{2}\right]$ (see Proposition III.5.2), $\left(\tilde{X}_{1}^{-2}\right)_{\tilde{X}_{2}}=\left(\tilde{X}_{1}^{-2}\right)_{\tilde{X}_{2}^{-1}} c$. Thus: $u_{\tilde{X}_{1}^{-1}}=$ $\tilde{X}_{2}^{2} \cdot\left(\tilde{X}_{1}^{-2}\right)_{\tilde{X}_{2}^{-1}} c=u^{-1} c$.

$\left(2_{a}\right)$ Since $\left[\tilde{X}_{1}^{2}, \tilde{X}_{2}^{2}\right]=c, u_{\tilde{X}_{2}^{-1}}=\left(\tilde{X}_{1}^{2}\right)_{\tilde{X}_{2}^{-2}} \cdot \tilde{X}_{2}^{-2}=c \tilde{X}_{1}^{2} \tilde{X}_{2}^{-2}=\tilde{X}_{2}^{-2} \cdot \tilde{X}_{1}^{2}$, and $u_{\tilde{X}_{2}^{-1}} \tilde{X}_{1}^{-1}=\left(\tilde{X}_{2}^{-2}\right)_{\tilde{X}_{1}^{-1}} \cdot \tilde{X}_{1}^{2}=\left(\tilde{X}_{1}^{-2}\right)_{\tilde{X}_{2}} \cdot \tilde{X}_{1}^{2}=c\left(\tilde{X}_{1}^{-2}\right)_{\tilde{X}_{2}^{-1}} \cdot \tilde{X}_{1}^{2}$. 
On the other hand, $u^{-1} u_{\tilde{X}_{2}^{-1}}=\tilde{X}_{2}^{2} \cdot\left(\tilde{X}_{1}^{-2}\right)_{\tilde{X}_{2}^{-1}} \cdot \tilde{X}_{2}^{-2} \cdot \tilde{X}_{1}^{2}=c\left(\tilde{X}_{1}^{-2}\right)_{\tilde{X}_{2}^{-1}} \cdot$ $\tilde{X}_{1}^{2}$. We get

$$
u_{\tilde{X}_{2}^{-1} \tilde{X}_{1}^{-1}}=u^{-1} u_{\tilde{X}_{2}^{-1}} .
$$

$\left(2_{b}\right)$ Using (1) and $\left(2_{a}\right)$, we can write

$$
\begin{aligned}
& u_{\tilde{X}_{1} \tilde{X}_{2}^{-1}}=\left(u^{-1} c\right)_{\tilde{X}_{2}^{-1}}=u_{\tilde{X}_{2}^{-1}}^{-1} \cdot c \\
& u_{\tilde{X}_{1} \tilde{X}_{2}^{-1} \tilde{X}_{1}^{-1}}=\left(u^{-1} c\right)_{\tilde{X}_{2}^{-1}} \tilde{X}_{1}^{-1}=u_{\tilde{X}_{2}^{-1}}^{-1} u \cdot c \\
& u_{\tilde{X}_{1}}^{-1} u_{\tilde{X}_{1} \tilde{X}_{2}^{-1}}=c u \cdot u_{\tilde{X}_{2}^{-1}}^{-1} c=u_{\tilde{X}_{2}^{-1}}^{-1} u \cdot c
\end{aligned}
$$

(we use $\left[u, u_{\tilde{X}_{2}^{-1}}\right]=\left[\left(\tilde{X}_{1}^{2}\right)_{\tilde{X}_{2}^{-1}} \cdot \tilde{X}_{2}^{-2}, \tilde{X}_{2}^{-2} \tilde{X}_{1}^{2}\right]=c \cdot c \cdot c=c$ ). Thus,

$$
u_{\tilde{X}_{1} \tilde{X}_{2}^{-1} \tilde{X}_{1}^{-1}}=u_{\tilde{X}_{1}}^{-1} u_{\tilde{X}_{1} \tilde{X}_{2}^{-1}}
$$

(3) Clearly, $\forall j \geq 4, u_{\tilde{X}_{j}}=u$. Consider $u_{\tilde{X}_{3}^{-1}}=\left(\tilde{X}_{1}^{2}\right)_{\tilde{X}_{2}^{-1} \tilde{X}_{3}^{-1}}\left(\tilde{X}_{2}^{-2}\right)_{\tilde{X}_{3}^{-1}}$.

Since $u$ can also be written as $u=\left(\tilde{X}_{1}^{2}\right)_{\tilde{X}_{2}^{-1}} \cdot \tilde{X}_{2}^{-2}=\tilde{X}_{2}^{-2} \cdot\left(\tilde{X}_{1}^{2}\right)_{\tilde{X}_{2}^{-1}} \cdot c=$ $\tilde{X}_{2}^{-2} \cdot\left(\tilde{X}_{1}^{2}\right)_{\tilde{X}_{2}}$, we have:

$$
\begin{aligned}
& u_{\tilde{X}_{3}^{-1}}=u \Leftrightarrow\left(\tilde{X}_{1}^{2}\right)_{\tilde{X}_{2}^{-1} \tilde{X}_{3}^{-1}} \cdot\left(\tilde{X}_{2}^{-2}\right)_{\tilde{X}_{3}^{-1}}=\tilde{X}_{2}^{-2} \cdot\left(\tilde{X}_{1}^{2}\right)_{\tilde{X}_{2}} \Leftrightarrow \tilde{X}_{2}^{2} \cdot\left(\tilde{X}_{1}^{2}\right)_{\tilde{X}_{2}^{-1} \tilde{X}_{3}^{-1}} \\
& =\left(\tilde{X}_{1}^{2}\right)_{\tilde{X}_{2}} \cdot\left(\tilde{X}_{2}^{2}\right)_{\tilde{X}_{3}^{-1}}
\end{aligned}
$$

which is true, because $\left\{\left(\tilde{X}_{1}\right)_{\tilde{X}_{2}}, \tilde{X}_{2},\left(\tilde{X}_{2}\right)_{\tilde{X}_{3}^{-1}},\left(\tilde{X}_{1}\right)_{\tilde{X}_{2}^{-1} \tilde{X}_{3}^{-1}}\right\}$ form a good quadrangle (see Fig. III.6).

\section{Construction of $\underline{\mathbf{G}}(\mathbf{n})$.}

For $n \geq 3$ we define the group $\underline{G}(n)$ as follows:

Generators: $s_{1}, u_{1}, u_{2}, \ldots, u_{n-1}$.

Relations:

$$
\begin{aligned}
& {\left[s_{1}, u_{i}\right]=1 \quad \forall i=1,3, \ldots n-1 ;\left[u_{i}, u_{j}\right]=1 \text { when }|i-j| \geq 2} \\
& {\left[s_{1}, u_{2}\right]=\left[u_{i}, u_{i+1}\right]=\left[u_{1}, u_{2}\right] \forall i=2,3, \ldots n-2} \\
& {\left[u_{1}, u_{2}\right]=\left[u_{1}, u_{2}\right]_{s_{1}}=\left[u_{1}, u_{2}\right]_{u_{i}} \forall i=1,2, \ldots, n-1} \\
& {\left[u_{1}, u_{2}\right]^{2}=1}
\end{aligned}
$$




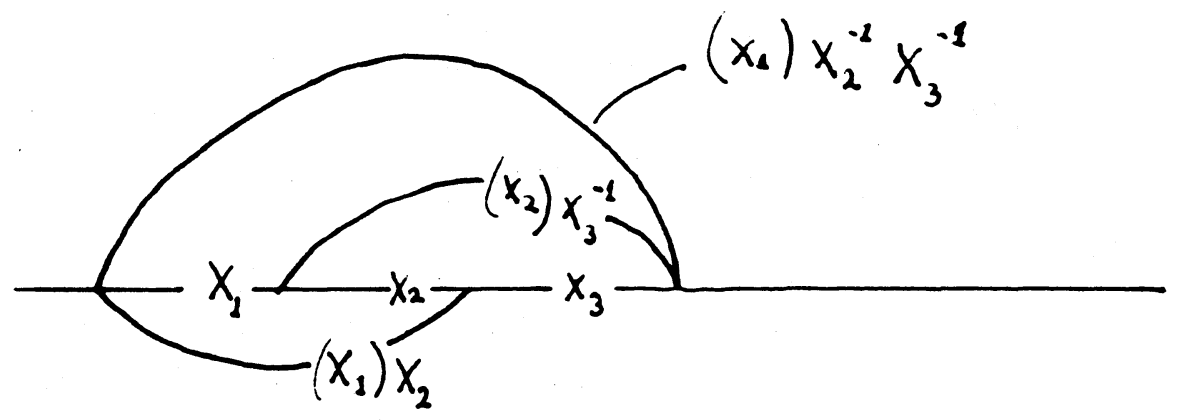

Figure III.6.

\section{Equivalent construction of $\underline{\mathbf{G}}(\mathbf{n})$.}

Consider a free abelian group $A(n)$ with generators $S_{1}, V_{1}, \ldots, V_{n-1}$ and a skew-symmetric $\mathbb{Z} / 2$ - valued bilinear form $Q(x, y)$ on $A(n)$ defined by: $Q\left(S_{1}, V_{i}\right)=0 \forall i=1,3, \ldots, n-1 ; Q\left(V_{i}, V_{j}\right)=0$ when $|i-j| \geq 2, Q\left(S_{1}, V_{2}\right)=$ $Q\left(V_{i}, V_{i+1}\right)=1 \forall i=1,2, \ldots, n-2$. One can check that there exists a unique central extension $G$ of $A(n)$ by $\mathbb{Z} / 2$ with $\mathrm{Ab}(G) \simeq A(n), G^{\prime} \simeq \mathbb{Z} / 2$ and such that $\forall x, y \in G[x, y]=Q(\bar{x}, \bar{y})$ where $(\bar{x}$ and $\bar{y}$ are the images of $x, y$ in $A(n))$.

Claim III.6.2. (1) The above central extension is isomorphic to $\underline{G}(n)$.

(2) $\mathrm{Ab}(\underline{G}(n))$ is a free abelian group with $n$ generators (i.e., $A(n))$ and $\underline{G}(n)^{\prime} \simeq \mathbb{Z} / 2$ (generated by $\left.\left[u_{1}, u_{2}\right]\right)$.

(3) The following formulas define a $\tilde{B}_{n}$-action on $\underline{G}(n)$ for $\left(\tilde{X}_{1}, \ldots\right.$, $\left.\tilde{X}_{n-1}\right)$, a standard set of generators in $\tilde{B}_{n}$, and $\nu=\left[u_{1}, u_{2}\right]$.

$$
\begin{aligned}
& \underline{\tilde{X}_{1-a c t i o n}} \quad \underline{\tilde{X}_{2} \text {-action }} \quad \underline{\tilde{X}_{k} \text {-action, } k \geq 3} \\
& s_{1} \rightarrow s_{1} \quad s_{1} \rightarrow u_{2} s_{1} \quad s_{1} \rightarrow s_{1} \\
& u_{1} \rightarrow u_{1}^{-1} \nu \quad u_{1} \rightarrow u_{2} u_{1} \quad u_{k-1} \rightarrow u_{k} u_{k-1} \\
& u_{2} \rightarrow u_{1} u_{2} \quad u_{2} \rightarrow u_{2}^{-1} \nu \quad u_{k} \rightarrow u_{k}^{-1} \nu \\
& u_{j} \rightarrow u_{j} \forall j \geq 3 \quad u_{3} \rightarrow u_{2} u_{3} ; \quad u_{k+1} \rightarrow u_{k} u_{k+1} \\
& u_{j} \rightarrow u_{j} \forall j \geq 4 \quad u_{j} \rightarrow u_{j} \forall j \neq k-1, k, k+1
\end{aligned}
$$

(4) Let $b \in \tilde{B}_{n}, y=\left(\tilde{X}_{1}\right)_{b}$. Then the $y^{2}$-action on $\underline{G}(n)$ coincides with the conjugation by $\left(s_{1}\right)_{b}$. 
(5) Let

(1.20)

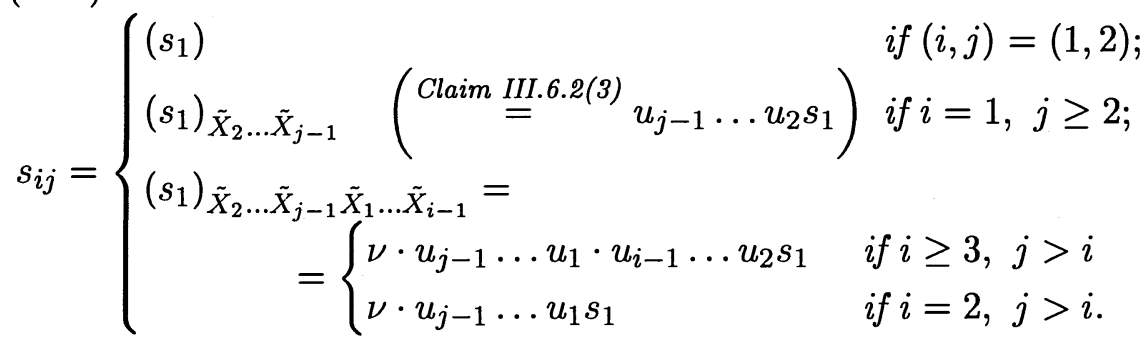

Then:

$$
\left[s_{i j}, s_{k l}\right]= \begin{cases}\nu, & \text { if }(\{i, j\} \cap\{k, l\})=1) \\ 1, & \text { otherwise. }\end{cases}
$$

(6) Let $\tilde{F}_{n-1}$ be the subgroup of $\underline{G}(n)$ generated by $\left(s_{n-1, n}, s_{n-2, n}, \ldots, s_{1 n}\right)$. $\tilde{B}_{n-1}$ acts on $\tilde{F}_{n-1}$ as follows:

$$
\begin{aligned}
\left(s_{j n}\right) \tilde{X}_{k} & =s_{j n} \quad j \neq k, k+1 ; \quad k=1 \ldots n-1 \\
\left(s_{k, n}\right) \tilde{X}_{k} & =s_{k+1, n} \\
\left(s_{k+1, n}\right) \tilde{X}_{k} & =s_{k n} \nu=s_{k+1, n} s_{k n} s_{k+1, n}^{-1} .
\end{aligned}
$$

This action is a $\tilde{B}_{n-1}$-action, where the action of the generators $\tilde{X}_{n-2}, \ldots \tilde{X}_{1}$ of $\tilde{B}_{n-1}$ correspond to standard Hurwitz moves on

$$
\left(s_{n-1, n}, s_{n-1, n}, \ldots, s_{1 n}\right) .
$$

(See the definition of Hurwitz moves in Chapter 0.)

(7) There is a natural chain of embeddings $\underline{G}(3) \subset \underline{G}(4) \subset \cdots \subset \underline{G}(n-$ 1) $\subset \underline{G}(n)$ corresponding to the chain: $\left(s_{1}, u_{1}, u_{2}\right) \subset\left(s_{1}, u_{1}, u_{2}, u_{3}\right) \subset$ $\ldots \subset\left(s_{1}, u_{1}, \ldots u_{n-1}\right)$.

Proof.

$(1),(2)$, and (3) are easy to verify.

(4) Consider first the case $b=I d$. From (3) we get for the $\tilde{X}_{1}^{2}$-action:

$$
s_{1} \rightarrow s_{1}, u_{1} \rightarrow u_{1}, u_{2} \rightarrow u_{2} \nu, u_{j} \rightarrow u_{j} \forall j \geq 3 \text {. }
$$

At the same time by the first construction:

$$
\left(s_{1}\right)_{s_{1}}=s_{1},\left(u_{1}\right)_{s_{1}}=u_{1},\left(u_{2}\right)_{s_{1}}=s_{1}^{-1} u_{2} s_{1}=u_{2} \nu,\left(u_{j}\right)_{s_{1}}=u_{j} \forall j \geq 3 .
$$


Thus $\tilde{X}_{1}^{2}$-action and $s_{1}$-conjugation coincide. Consider now any $b \in \tilde{B}_{n}$ and any $g \in \underline{G}(n)$. Let $h=g_{b^{-1}}$. We have:

$$
g_{\left(\tilde{X}_{1}^{2}\right)_{b}}=g_{b^{-1} \tilde{X}_{1}^{2} b}=\left((h)_{\tilde{X}_{1}^{2}}\right)_{b}=\left(h_{s_{1}}\right)_{b}=\left(h_{b}\right)_{\left(s_{1}\right)_{b}}=g_{\left(s_{1}\right)_{b}} .
$$

$(5),(6),(7)$ are easy to verify.

Lemma III.6.3. Let $n \geq 3$.

Let $\left\{X_{1}, \ldots, X_{n-1}\right\}$ be a frame of $B_{n}$.

Let

$$
Z_{i j}= \begin{cases}X_{1} & \text { if }(i, j)=(1,2) \\ \left(X_{1}\right)_{X_{2} \ldots X_{j-1}} & \text { if } i=1, j \geq 3 \\ \left(X_{1}\right)_{X_{2} \ldots X_{j-1} X_{1} \ldots X_{i-1}} & \text { if } i \geq 2, j>i .\end{cases}
$$

Let $\tilde{Z}_{i j}$ be the image of $Z_{i j}$ in $\tilde{B}_{n}$.

Consider $\underline{G}(n)$ as a $B_{n}$-group as in Claim III.6.2.

Then there exists a unique $\tilde{B}_{n}$-surjection $\Lambda_{n}: \tilde{P}_{n} \rightarrow \underline{G}(n)$ with $\Lambda_{n}\left(\tilde{X}_{1}^{2}\right)=$ $s_{1}$ and $\Lambda_{n}\left(\tilde{Z}_{i j}^{2}\right)=s_{i j}$ for $1 \leq i<j \leq n$.

Proof. Use induction on $n$.

For $n=3, \Lambda_{3} \tilde{P}_{3} \rightarrow \underline{G}(3)$ must be defined by $\lambda_{3}\left(\tilde{Z}_{i j}^{2}\right)=s_{i j}, 1 \leq i<j \leq 3$. One can check directly that $\Lambda_{3}$ is well defined, and that it is a $\tilde{B}_{3}$-surjection. Uniqueness of such $\Lambda_{3}$ is evident. exists.

Assume now that $n \geq 4$ and that the desired $\Lambda_{n-1}: \tilde{P}_{n-1} \rightarrow \underline{G}(n-1)$

Considering $\left(X_{1}, X_{2}\right) \subset\left(X_{1}, X_{2}, X_{3}\right) \subset \ldots \subset\left(X_{1}, \ldots, X_{n-1}\right)$, we get a chain of embeddings $B_{3} \subset B_{4} \subset \ldots \subset B_{n}$ and the corresponding chain $P_{3} \subset P_{4} \subset \ldots \subset P_{n}$. To the latter corresponds a chain of homomorphisms: $\tilde{P}_{3} \stackrel{i_{3}}{\longrightarrow} \tilde{P}_{4} \stackrel{i_{4}}{\longrightarrow} \ldots \rightarrow \tilde{P}_{n-1} \stackrel{i_{n-1}}{\longrightarrow} \tilde{P}_{n}$, where $\tilde{P}_{3}$ is obtained (by definition) from $P_{3}$ by adding the relations: $\left[\tilde{Z}_{12}^{2}, \tilde{Z}_{23}^{2}\right]=\left[\tilde{Z}_{12}^{2}, \tilde{Z}_{13}^{2}\right]=\left[\tilde{Z}_{23}^{2}, \tilde{Z}_{13}^{2}\right]$ and $\left[\tilde{Z}_{12}^{2}, \tilde{Z}_{23}^{2}\right]^{2}=$ 1.

It is known that the set $\left\{Z_{i j}^{2}, 1 \leq i<j \leq n\right\}$ generates $P_{n}$, and $P_{n} \simeq$ $P_{n-1} \ltimes F_{n-1}$, where $P_{n-1}$ is the subgroup of $P_{n}$ generated by $\left\{Z_{i j}^{2}, 1 \leq i<\right.$ $j \leq n-1\}, F_{n-1}$ is a free subgroup of $P_{n}$ generated by $\left\{Z_{i n}^{2}, 1 \leq i \leq n-1\right\}$, and the semi-direct product $P_{n-1} \ltimes F_{n-1}$ is defined according to the $P_{n-1^{-}}$ action on $F_{n-1}$ which comes from the $B_{n-1}$-action by conjugation (using $\left.B_{n-1} \subset B_{n} \supset P_{n}\right)$. The latter coincides with the standard $B_{n-1}$-action on $F_{n-1}$ (the generators $X_{n-2}, \ldots, X_{1}$ of $B_{n-1}$ correspond to standard Hurwitz moves on $\left(Z_{n-1, n}^{2}, Z_{n-2, n}^{2}, \ldots, Z_{1 n}^{2}\right)$ (see [MoTe4], Chapter 4). 
Using canonical $P_{n-1} \rightarrow \tilde{P}_{n-1}$, we obtain from $\Lambda_{n-1}$ a $B_{n-1}$-surjection $\hat{\Lambda}_{n-1}: P_{n-1} \rightarrow \underline{G}(n)$. For the free subgroup $F_{n-1}$ of $P_{n}$ generated by $\left\{Z_{i n}^{2}, n-1 \leq i \leq 1\right\}$ define $\mu_{n-1}: F_{n-1} \rightarrow \underline{G}(n)$ by $\mu_{n-1}\left(Z_{i n}^{2}\right)=s_{i n}$. Considering $P_{n}$ as $P_{n-1} \ltimes F_{n-1}$, we define $\hat{\Lambda}_{n}: P_{n} \rightarrow \underline{G}(n)$ which on $P_{n-1}$ coincides with $\hat{\Lambda}_{n-1}: P_{n-1} \rightarrow \underline{G}(n-1) \subset \underline{G}(n)$ (see Claim III.6.2(7)) and on $F_{n-1}$ coincides with $\mu_{n-1}: F_{n-1} \rightarrow \underline{G}(n)$. To show that such $\hat{\Lambda}_{n}$ exists one has to check the following:

1) The conjugation of $\mu_{n-1}\left(F_{n-1}\right)$ by elements of $\hat{\Lambda}_{n-1}\left(P_{n-1}\right)(\subset \underline{G}(n))$ coincides with the $P_{n-1}$-action defined by $P_{n-1} \subset B_{n-1} \subset B_{n} \rightarrow \tilde{B}_{n}$ and the given $\tilde{B}_{n}$-action on $\underline{G}(n)$. That is, $\forall f \in \mu_{n-1}\left(F_{n-1}\right)$ and $\forall h$ of the form $\hat{\Lambda}_{n-1}(\tilde{Y})\left(Y \in P_{n-1}\right)$ we must have $h^{-1} f h=f_{\tilde{Y}}$.

2) The $P_{n-1}$-action on $\mu_{n-1}\left(F_{n-1}\right)$ (defined by $P_{n-1} \subset B_{n-1} \subset$ $B_{n} \rightarrow \tilde{B}_{n}$ and the given $\tilde{B}_{n}$-action on $\left.\underline{G}(n)\right)$ comes from $B_{n-1}$-action on $\mu_{n-1}\left(F_{n-1}\right)$ in which $X_{n-2}, \ldots X_{1}$ correspond to the standard Hurwitz moves on $\left(s_{n-1, n}, s_{n-2, n}, \ldots s_{1 n}\right)$.

Proof of 1). Since $\forall b \in B_{n-1}, \hat{\Lambda}_{n-1}\left(\left(X_{1}^{2}\right)_{b}\right)=\left(s_{1}\right)_{b}$ we see from Claim III.6.2 that $\forall f \in \mu_{n-1}\left(F_{n-1}\right), \quad f_{\hat{\Lambda}_{n-1}\left(\left(X_{1}^{2}\right)_{b}\right)}=\left(f_{s_{1}}\right)_{b}=f_{\left(\tilde{X}_{1}^{2}\right)_{b}}$. Since $P_{n-1}$ is generated by $\left\{Z_{i j}^{2}, 1 \leq i<j \leq n-1\right\}$, i.e., by $\left\{\left(X_{1}^{2}\right)_{b}, b \in B_{n}\right\}$ we get 1$)$.

Proof of 2). It follows immediately from Claim III.6.2(6).

Thus, 1) and 2) are true and we can extend $\hat{\Lambda}_{n-1}, \mu_{n-1}$ to a homomorphism $\hat{\Lambda}_{n}: P_{n}\left(=P_{n-1} \ltimes F_{n-1}\right) \rightarrow \underline{G}(n)$ such that for $1 \leq i<j \leq n-1$ $\hat{\Lambda}_{n}\left(Z_{i j}^{2}\right)=\hat{\Lambda}_{n-1}\left(Z_{i j}^{2}\right)=s_{i j}$, and for $1 \leq i \leq n-1 \hat{\Lambda}_{n}\left(Z_{i n}^{2}\right)=\mu_{n-1}\left(Z_{i n}^{2}\right)=s_{i n}$, in short $\hat{\Lambda}_{n}\left(Z_{i j}^{2}\right)=s_{i j}$ for $1 \leq i<j \leq n$.

Using induction, one can check directly that $\hat{\Lambda}_{n}$ is a $B_{n}$-homomorphism (recall that by Claim III.6.2 we have explicit formulas for $s_{i j}$ 's).

Because $s_{1 n}=u_{n-1} \cdot u_{2} s_{1}$ (by 1.20), that is, $u_{n-1}=s_{1 n}\left(u_{n-2} \ldots u_{2} s\right)^{-1}$, we see that $\underline{G}(n)$ is generated by $\underline{G}(n-1)=\hat{\Lambda}_{n}\left(P_{n-1}\right)\left(=\hat{\Lambda}_{n-1}\left(P_{n-1}\right)\right)$ and $s_{1 n}=\hat{\Lambda}_{n}\left(Z_{1 n}^{2}\right)$. Therefore $\hat{\Lambda}_{n}$ is a $B_{n}$-surjection.

Let $N=\operatorname{ker}\left(B_{n} \rightarrow \tilde{B}_{n}\right)\left(=\operatorname{ker}\left(P_{n} \rightarrow \tilde{P}_{n}\right)\right)$. Let $T=X_{1}^{2} X_{3}^{2} X_{2}^{-2} Z_{14}^{-2}$. Clearly, $N$ is generated by $\left\{T_{b}, b \in B_{n}\right\}$. We have $\hat{\Lambda}_{n}(T)=\hat{\Lambda}_{4}(T)=s_{1} \cdot s_{34}$. $s_{23}^{-1} s_{14}^{-1} \stackrel{\text { Claim III.6.2 }}{=} s_{1} \cdot \eta u_{3} u_{2} u_{1} \cdot u_{2} s_{1} \cdot s_{1}^{-1} u_{1}^{-1} u_{2}^{-1} \eta \cdot s_{1}^{-1} u_{2}^{-1} u_{3}^{-1}=s_{1} \eta u_{3} u_{2}$. $s_{1}^{-1} u_{2}^{-1} u_{3}^{-1}=$ Id. Since $\hat{\Lambda}_{n}$ is a $B_{n}$-homomorphism, we get $\hat{\Lambda}_{n}\left(T_{b}\right)=$ Id $\forall b \in B_{n}$, and thus $\hat{\Lambda}_{n}(N)=$ Id. Hence $\hat{\Lambda}_{n}$ defines canonically a $\tilde{B}_{n}$-surjection $\Lambda_{n}: \tilde{P}_{n} \rightarrow \underline{G}(n)$ with $\Lambda_{n}\left(\tilde{X}_{1}^{2}\right)=s_{1}$. 
Uniqueness of such $\Lambda_{n}$ follows from the fact that $\tilde{P}_{n}$ is generated by the $B_{n}$-orbit of $\tilde{X}_{1}^{2}$.

Theorem III.6.4. There exists a unique $\tilde{B}_{n}$-isomorphism $\Lambda_{n}: \tilde{P}_{n} \rightarrow \underline{G}(n)$ with $\Lambda_{n}\left(\tilde{X}_{1}^{2}\right)=s_{1}$. In particular:

(1) $\mathrm{Ab} \tilde{P}_{n}$ is a free abelian group with $n$ generators, $\tilde{P}_{n}^{\prime} \simeq \mathbb{Z} / 2$ (generated by $\left.c=\left[\tilde{X}_{1}^{2}, \tilde{X}_{2}^{2}\right]\right)$;

(2) $\tilde{P}_{n, 0}$ is $\tilde{B}_{n}$-isomorphic to the subgroup $G_{0}(n)$ of $\underline{G}(n)$, generated by $u_{1}, \ldots, u_{n-1}, \mathrm{Ab} \tilde{P}_{n, 0}$ is a free abelian group with $n-1$ generators $\left\{\xi_{1}, \ldots, \xi_{n-1}\right\}, \tilde{P}_{n, 0}^{\prime} \simeq \mathbb{Z} / 2$, generated by $c=\left[\xi_{1}, \xi_{2}\right] ; \quad\left(\nu \in G_{0}(n)\right.$ corresponding to $\left.c \in \tilde{P}_{n, 0}\right)$.

(3) $\tilde{P}_{n, 0}$ is a primitive $\tilde{B}_{n}$-group generated by the $\tilde{B}_{n}$-orbit of a prime element $u=\tilde{X}^{2} \tilde{Y}^{-2}$, where $\tilde{X}, \tilde{Y}$ are adjacent half-twists in $\tilde{B}_{n}, \tilde{T}=$ $\tilde{X} \tilde{Y} \tilde{X}^{-1}$ is a supporting half-twist for $u$.

Proof. Clearly, $\tilde{P}_{n, 0}$ is generated by $\left\{\tilde{X}_{1}^{2} \tilde{Z}_{i j}^{-2}, l \leq i<j \leq n\right\}$. Because $\tilde{X}_{1}^{2} \cdot \tilde{Z}_{i j}^{-2}=\tilde{X}_{1}^{2} \tilde{Z}_{1 i}^{-2} \cdot \tilde{Z}_{1 i}^{2} \cdot \tilde{Z}_{i j}^{-2}$ and both $\tilde{X}_{1}^{2} \tilde{Z}_{1 i}^{-2}, \tilde{Z}_{1 i}^{2} \cdot \tilde{Z}_{i j}^{-2}$ are conjugates of $u$, we see that $\tilde{P}_{n, 0}$ is generated by the $\tilde{B}_{n^{-}}$orbit of $u$, and since $u$ is a prime element of $P_{n, 0}$, this means that $\tilde{P}_{n, 0}$ is a primitive $\tilde{B}_{n}$-group. By Lemma III.6.1, the s.h.t. of $u$ is $Y^{-1} X Y$. Thus, we proved (3).

Polarize each $X_{i}$ (and $\left.\tilde{X}_{i}\right)$ according to the sequence $\left(X_{1}, \ldots X_{n-1}\right)$ (the "end" of $X_{i}=$ the "origin" of $\left.X_{i+1}\right)$. By Proposition III.3.3 $\forall i=1, \ldots, n-$ $1 \exists$ unique prime element $\xi_{i}=L_{\left\{u, \tilde{X}_{1}\right\}}\left(\tilde{X}_{i}\right) \in \tilde{P}_{n, 0}$ such that $\left\{\xi_{i}, \tilde{X}_{i}\right\}$ is coherent with $\left\{u, \tilde{X}_{1}\right\}$. Clearly $\xi_{1}=u$.

By Proposition III.4.1 we have $\forall i=1, \ldots, n-1$ :

$$
\left(\xi_{i}\right)_{\tilde{X}_{i}^{-1}}=\xi_{i}^{-1} c ;\left(\xi_{i}\right)_{\tilde{X}_{i-1}^{-1}}=\xi_{i} \xi_{i-1} ;=\left(\xi_{i}\right)_{\tilde{X}_{i+1}^{-1}}=\xi_{i} \xi_{i+1} .
$$

It is clear also that $\forall j \neq i, i-1, i+1$

$$
\left(\xi_{i}\right)_{\tilde{X}_{j}}=\xi_{i}
$$

We see from (1.22), (1.23) that the subgroup of $\tilde{P}_{n, 0}$ generated by $\left(\xi_{1}, \ldots, \xi_{n-1}\right)$ is closed under the $\tilde{B}_{n}$-action. Since $\tilde{P}_{n, 0}$ is generated by the $\tilde{B}_{n}$-orbit of $u=\xi_{1}$, we conclude that $\tilde{P}_{n, 0}$ is generated by $\left(\xi_{1}, \ldots, \xi_{n-1}\right)$. This implies that $\tilde{P}_{n}$ is generated by $\left(\tilde{X}_{1}^{2}, \xi_{1}, \xi_{2}, \ldots, \xi_{n-1}\right)$. 

that

We have $\xi_{2}=L_{\left\{u, \tilde{X}_{1}\right\}}\left(\tilde{X}_{2}\right)=\left(\xi_{1}\right)_{\tilde{X}_{2}^{-1} \tilde{X}_{1}^{-1}}=\left(\tilde{X}_{1}^{-2}\right)_{\tilde{X}_{2}} \cdot \tilde{X}_{1}^{2}$, which implies

$$
\left[\tilde{X}_{1}^{2}, \xi_{2}\right]=c
$$

By Lemma III.5.1 we have

$$
\left[\xi_{i}, \xi_{j}\right]= \begin{cases}c & \text { if }|i-j|=1 \\ 1 & \text { if }|i-j| \geq 2\end{cases}
$$

Observe also that

$$
\left(\tilde{X}_{1}^{2}\right)_{\tilde{X}_{2}}=\xi_{2} \cdot \tilde{X}_{1}^{2}
$$

Formulas (1.22)-(1.26) show that we can define a $\tilde{B}_{n}$-homomorphism $M_{n}: \underline{G}(n) \rightarrow \tilde{P}_{n}$ with $M_{n}\left(s_{1}\right)=\tilde{X}_{1}^{2}, M_{n}\left(u_{i}\right)=\xi_{i}, i=1, \ldots, n-1$. (See Claim III.6.2.)

Since $\tilde{P}_{n}$ is generated by the $\tilde{B}_{n}$-orbit of $\tilde{X}_{1}^{2}$ and $\underline{G}(n)$ is generated by the $\tilde{B}_{n}$-orbit of $s_{1}$, we conclude that $\Lambda_{n}$ and $M_{n}$ are inverses of each other.

\section{III.7. Criterion for prime element.}

Proposition III.7.1. Assume $n \geq 5$. Let $G$ be a $\tilde{B}_{n}$-group,

$$
\left(\tilde{X}_{1}, \tilde{X}_{2}, \ldots, \tilde{X}_{n-1}\right)
$$

be a standard base of $\tilde{B}_{n}$. Let $S$ be an element of $G$ with the following properties:

(0) $G$ is generated by $\left\{S_{b}, b \in \tilde{B}_{n}\right\}$;

(1 $\left.1_{a}\right) S_{\tilde{X}_{2}^{-1} \tilde{X}_{1}^{-1}}=S^{-1} S_{\tilde{X}_{2}^{-1}} ;$

(1b) $S_{\tilde{X}_{1} \tilde{X}_{2}^{-1} \tilde{X}_{1}^{-1}}=S_{\tilde{X}_{1}}^{-1} S_{\tilde{X}_{1} \tilde{X}_{2}^{-1}}$;

(2) For $\tau=S S_{\tilde{X}_{1}^{-1}}, T=S_{\tilde{X}_{2}^{-1}}$ we have:

(2a) $\tau_{\tilde{X}_{1}^{2}}=\tau$

$\left(2_{b}\right) \tau_{T}=\tau_{\tilde{X}_{1}}^{-1}$ 
(3) $S_{\tilde{X}_{j}}=S \forall j \geq 3$;

(4) $S_{c}=S$, where $c=\left[\tilde{X}_{1}^{2}, \tilde{X}_{2}^{2}\right]$.

Then $S$ is a prime element of $G, \tilde{X}_{1}$ is a supporting half-twist of $S$ and $\tau$ is the corresponding central element. In particular, $\tau^{2}=1, \tau \in \operatorname{Center}(G)$, $\tau_{b}=\tau \forall b \in \tilde{B}_{n}$.

Proof. The proof includes several lemmas. From Theorem III.5.2, $c \in$ Center $\left(\tilde{B}_{n}\right), c^{2}=1$. From Theorem III.6.4 it follows that $\tilde{P}_{n}^{\prime}$ is generated by $c$. $\tilde{P}_{n}^{\prime}$ is a normal subgroup of $\tilde{B}_{n}$. Denote by $\tilde{\tilde{B}}_{n}=\tilde{B}_{n} / \tilde{P}_{n}^{\prime}$, $\tilde{\tilde{P}}_{n}=\tilde{P}_{n} / \tilde{P}_{n}^{\prime}=\operatorname{Ab} \tilde{P}_{n}$. $\tilde{\tilde{P}}_{n}$ is a commutative group. We have $\tilde{\tilde{\psi}}_{n}: \tilde{\tilde{B}}_{n} \rightarrow S_{n}$. By abuse of notaion we use $\psi_{n}$ for $\tilde{\psi}_{n}$. Let $Y \in B_{n}$. By abuse of notation we denote the image of $Y$ in $\tilde{B}_{n}$ or in $\tilde{\tilde{B}}_{n}$ or in $\tilde{\tilde{P}}_{n}$ by the same symbol $\tilde{Y}$. It is clear that $\tilde{B}_{n}$ acts on $\tilde{\tilde{P}}_{n}$ (through conjugations) as the symmetric group $S_{n}=\tilde{B}_{n} / \tilde{P}_{n}$.

Since $S_{c}=S$ and $c \in$ Center $\tilde{B}_{n}$, we have $\forall b \in \tilde{B}_{n}\left(S_{b}\right)_{c}=S_{b c}=$ $\left(S_{c}\right)_{b}=S_{b}$. Since $G$ is generated by $\left\{S_{b}, b \in \tilde{B}_{n}\right\}$ we have $\forall g \in G g_{c}=g$. In particular, we conclude that $\tilde{B}_{n}$ acts on $G$ as its quotient $\tilde{\tilde{B}}_{n}$; in other words, $G$ is a $\tilde{\tilde{B}}_{n}$-group.

Let $(D, K)$ be a model for $B_{n}, K=\left\{a_{1}, \ldots, a_{n}\right\}, B_{n}=B_{n}[D, K]$. Take any $a_{i_{1}}, a_{i_{2}} \in K$. Let $\gamma_{1}, \gamma_{2}$ be two different simple paths in $D-\left(K-a_{i_{1}}-a_{i_{2}}\right)$ connecting $a_{i_{1}}$ with $a_{i_{2}}$, let $H\left(\gamma_{1}\right), H\left(\gamma_{2}\right)$ be the half-twists corresponding to $\gamma_{1}, \gamma_{2}$, and let $\tilde{H}\left(\gamma_{1}\right), \tilde{H}\left(\gamma_{2}\right)$ be the images of $H\left(\gamma_{1}\right), H\left(\gamma_{2}\right)$ in $\tilde{\tilde{B}}_{n}$.

Lemma 1. Let $\gamma_{1}, \gamma_{2}$ be 2 simple paths in $D-\left\{K-a_{i_{1}}-a_{i_{2}}\right\}$ connecting $a_{i_{1}}$ with $a_{i_{2}}$. Then: $\tilde{H}\left(\gamma_{1}\right)^{2}=\tilde{H}\left(\gamma_{2}\right)^{2}$.

Proof of Lemma 1. Choose a frame of $B_{n}\left(Y_{1}, \ldots, Y_{n-1}\right)$ s.t. $Y_{1}=H\left(\gamma_{1}\right)$. Let $b \in B_{n}$ s.t. $\gamma_{2}=\left(\gamma_{1}\right) b$, that is $H\left(\gamma_{1}\right)_{b}=H\left(\gamma_{2}\right)$. Let $\tilde{Y}_{i}$ be the image of $Y_{i}$ in $\tilde{\tilde{B}}_{n}$.

Let $\sigma_{1}$ be the image of $b$ in $S_{n}$. Since $\left(a_{i}\right) b=a_{i},\left(a_{j}\right) b=a_{j}, \sigma_{1} \in$ $\operatorname{Stab}(i) \cap \operatorname{Stab}(j)$ in $S_{n}$. The subgroup of $\tilde{\tilde{B}}_{n}$ generated by $\tilde{Y}_{3}, \ldots, \tilde{Y}_{n-1}$ is mapped by $\tilde{\tilde{\psi}}_{n}: \tilde{\tilde{B}}_{n} \rightarrow S_{n}$ onto $\operatorname{Stab}(i) \cap \operatorname{Stab}(j)$. Choose $\tilde{b}_{1}$ in this subgroup with its image in $S_{n}$ equal to $\sigma_{1}$. Clearly, $\left(\tilde{Y}_{1}\right)_{\tilde{b}_{1}}=\tilde{Y}_{1}$. Since the image of $\tilde{b}_{1}^{-1} \tilde{b}$ in $S_{n}$ is equal to $\sigma_{1}^{-1} \sigma_{1}=\mathrm{Id}$, we have $\tilde{b}_{1}^{-1} \tilde{b} \in \tilde{\tilde{P}}_{n}$. Since $\tilde{\tilde{P}}_{n}$ 
is commutative when considering $\tilde{Y}_{1}^{2}$ as an element of $\tilde{\tilde{P}}_{n},\left(\tilde{Y}_{1}^{2}\right)_{b_{1}^{-1} \tilde{b}}=\tilde{Y}_{1}^{2}$. Thus, we have

$$
\tilde{H}\left(\gamma_{2}\right)^{2}=\tilde{H}\left(\gamma_{1}\right)_{\tilde{b}}^{2}=\left(\tilde{Y}_{1}^{2}\right)_{\tilde{b}_{1} \tilde{b}_{1}^{-1} \tilde{b}}=\left(\tilde{Y}_{1}^{2}\right)_{\tilde{b}_{1}^{-1} \tilde{b}}=\tilde{Y}_{1}^{2}=\tilde{H}\left(\gamma_{1}\right)^{2} \quad \square \text { Lemma } 1
$$

Definition. $f_{i j}$

$\forall i, j \in(1, \ldots, n), i \neq j$, we define $f_{i j} \in \tilde{\tilde{P}}_{n}$ as follows: Take any simple path $\gamma$ in $D-\left(K-a_{i}-a_{j}\right)$ connecting $a_{i}$ with $a_{j}$. Let $f_{i j}=\tilde{H}(\gamma)^{2}$. Lemma 1 shows that this definition does not depend on the choice of $\gamma$. We choose for $i<j$ :

$$
f_{i j}= \begin{cases}\left(\tilde{X}_{i}^{2}\right)_{X_{i+1}} \cdots X_{j-1} & 2<j-i \\ \tilde{X}_{i}^{2} & i+1=j .\end{cases}
$$

It is clear that for $\sigma_{1}$ the image in $S_{n}$ of $b \in \tilde{B}_{n}$ we have:

$$
\left(f_{i j}\right)_{b}=f_{(i) \sigma_{1},(j) \sigma_{1}} .
$$

It is clear from our choice of $\gamma$ for $f_{i j}=\tilde{H}(\gamma)^{2}$ that:

$$
\psi_{n}(\tilde{H}(\gamma))=(i, j)
$$

It will be convenient to use the following notation for $g \in G$ and $b \in \tilde{\tilde{B}}_{n}$ :

Notation. $\quad[g, b]$ : For $g \in G, b \in \tilde{\tilde{B}}_{n}$ and the action of $\tilde{\tilde{B}}_{n}$ on $G$, we denote $[g, b]=g \cdot g_{b^{-1}}^{-1}$.

One can check that:

$$
\begin{aligned}
& g_{b}=g \Leftrightarrow[g, b]=1 \\
& {[g, b]_{z}=g_{z}\left(g_{z}\right)_{b_{z}^{-1}}^{-1}} \\
& {\left[g^{-1}, b\right]=[g, b]_{g}^{-1}} \\
& {\left[g, b^{-1}\right]=[g, b]_{b}^{-1}} \\
& {\left[g_{1} g_{2}, b\right]=\left[g_{2}, b\right]_{g_{1}^{-1}} \cdot\left[g_{1}, b\right]} \\
& {\left[g, b_{1} b_{2}\right]=\left[g, b_{1}\right] \cdot\left[g, b_{2}\right]_{b_{1}^{-1}}}
\end{aligned}
$$

Notation. derline $Q_{b, l, m}$

$\forall b \in \tilde{\tilde{B}}_{n}, \quad \forall l, m \in(1, \ldots, n), l \neq m$, we denote $Q_{b, l, m}=\left[S_{b}, f_{l m}^{-1}\right]$. 
Lemma 2. (i) Let $b \in \tilde{\tilde{B}}_{n}$ be such that $(\{1,2\}) \psi_{n}(b) \cap\{l, m\}=\emptyset$. Then $Q_{b, l, m}=1$.

(ii) Let $Q=Q_{\mathrm{Id}, 1,3}=\left[S, f_{13}^{-1}\right]$. Then $Q_{\tilde{X}_{2}^{-1}}=Q$.

\section{Proof of Lemma 2.}

(i) Let $\left\{l_{1}, m_{1}\right\}=(\{l, m\}) \psi_{n}(b)^{-1}$. So $\left(f_{l m}\right)_{b^{-1}}=f_{l_{1} m_{1}}$. We have $\{1,2\} \cap$ $\left\{l_{1}, m_{1}\right\}=\emptyset$, that is, $3 \leq l_{1}, 3 \leq m_{1}$. By our choice, $f_{l m}$ is a product o $X_{j}$ for $j \geq 3$. Thus, using property (3) of $S$ we get $S_{f_{l_{1}, m_{1}}}=S$. In other words, $\left[S, f_{l_{1}, m_{1}}^{-1}\right]=1$. We get

$$
\left(Q_{b, l, m}\right)_{b^{-1}}=\left[S_{b}, f_{l m}^{-1}\right]_{b^{-1}}=\left[S,\left(f_{l m}^{-1}\right)_{b^{-1}}\right]=\left[S, f_{l_{1}, m_{1}}^{-1}\right]=1,
$$

and so $Q_{b, l, m}=1$.

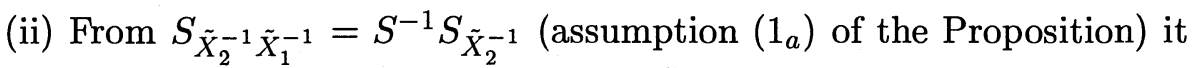
follows that $S_{\tilde{X}_{2}^{-1}}=S S_{\tilde{X}_{2}^{-1}} \tilde{X}_{1}^{-1}$. Applying $\tilde{X}_{3}^{-1}$, we get

$$
S_{\tilde{X}_{2}^{-1} \tilde{X}_{3}^{-1}}=S S_{\tilde{X}_{2}^{-1} \tilde{X}_{1}^{-1} \tilde{X}_{3}^{-1}}
$$

which, after applying $\tilde{X}_{2}^{-1}$, gives:

$$
S_{\tilde{X}_{2}^{-1} \tilde{X}_{3}^{-1} \tilde{X}_{2}^{-1}}=S_{\tilde{X}_{2}^{-1}} S_{\tilde{X}_{2}^{-1} \tilde{X}_{1}^{-1} \tilde{X}_{3}^{-1} \tilde{X}_{2}^{-1}}
$$

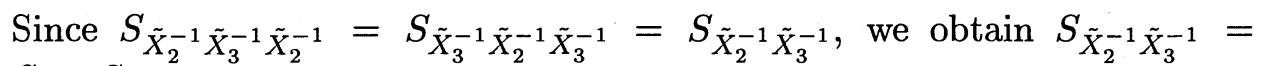
$S_{\tilde{X}_{2}^{-1}} S_{\tilde{X}_{2}^{-1} \tilde{X}_{1}^{-1} \tilde{X}_{3}^{-1} \tilde{X}_{2}^{-1}, \text { or }}$

$$
S_{\tilde{X}_{2}^{-1}}=S_{\tilde{X}_{2}^{-1} \tilde{X}_{3}^{-1}} S_{\tilde{X}_{2}^{-1} \tilde{X}_{1}^{-1} \tilde{X}_{3}^{-1} \tilde{X}_{2}^{-1}}
$$

Let $b_{1}=\tilde{X}_{2}^{-1} \tilde{X}_{1}^{-1} \tilde{X}_{3}^{-1} \tilde{X}_{2}^{-1}$. Observing that $\left(f_{13}\right)_{\tilde{X}_{2}^{-1}}=f_{12}$, we get from (1.28): $Q_{\tilde{X}_{2}^{-1}}=\left[S_{\tilde{X}_{2}^{-1}},\left(f_{13}^{-1}\right)_{\tilde{X}_{2}^{-1}}\right]=\left[S_{\tilde{X}_{2}^{-1} \tilde{X}_{3}^{-1}} S_{b_{1}}^{-1}, f_{12}^{-1}\right]$. Thus:

$$
Q_{\tilde{X}_{2}^{-1}}=\left[S_{b_{1}}^{-1}, f_{12}^{-1}\right]_{S_{\tilde{X}_{2}^{-1} \tilde{X}_{3}^{-1}}} \cdot\left[S_{\tilde{X}_{2}^{-1} \tilde{X}_{3}^{-1}}, f_{12}^{-1}\right] .
$$

Since $\psi_{n}\left(b_{1}\right)=\left(\begin{array}{lll}2 & 3\end{array}\right)\left(\begin{array}{ll}1 & 2\end{array}\right)\left(\begin{array}{lll}3 & 4\end{array}\right)\left(\begin{array}{l}2 \\ 3\end{array}\right)$ (products of transpositions), $(\{1,2\}) \psi_{n}\left(b_{n}\right)=\{3,4\}$. Since $\{3,4\} \cap\{1,2\}=\emptyset$, we get from (i) that $Q_{b_{1}, 1,2}=1$. Thus, $\left[S_{b_{1}}^{-1}, f_{12}^{-1}\right]=\left[S_{b_{1}}, f_{12}^{-1}\right]_{S_{b_{1}}}^{-1}=\left(Q_{b_{1}, 1,2}^{-1}\right)_{S_{b_{1}}}$. (1.29) now gives:

$$
Q_{\tilde{X}_{2}^{-1}}=\left[S_{\tilde{X}_{2}^{-1}} \tilde{X}_{3}^{-1}, f_{12}^{-1}\right] .
$$




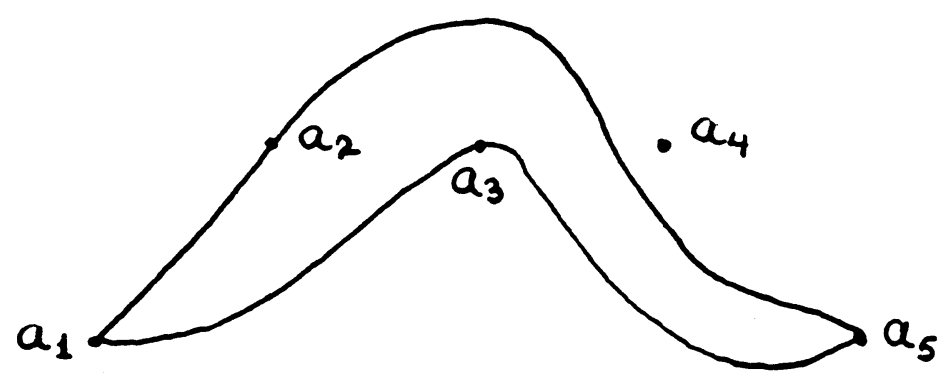

Figure III.7.1.

Consider a quadrangle formed by $\left\{a_{1}, a_{2}, a_{3}, a_{5}\right\}$, as in Fig. III.7.1. By Lemma III.1.2, we can write in $\tilde{\tilde{P}}_{n}: f_{35} f_{12}=f_{25} f_{13}$, or $f_{12}=f_{35}^{-1} f_{25} f_{13}$, $f_{12}^{-1}=f_{13}^{-1} f_{25}^{-1} f_{35}$.

From (1.30) we get:

$$
Q_{\tilde{X}_{2}^{-1}}=\left[S_{\tilde{X}_{2}^{-1} \tilde{X}_{3}^{-1}}, f_{13}^{-1} f_{25}^{-1} f_{35}\right]=\left[S_{\tilde{X}_{2}^{-1} \tilde{X}_{3}^{-1}} f_{13}^{-1}\right] \cdot\left[S_{\tilde{X}_{2}^{-1} \tilde{X}_{3}^{-1}}, f_{25}^{-1} f_{35}\right]_{f_{13}}
$$

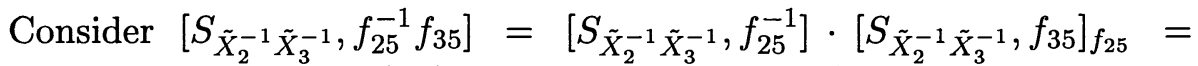

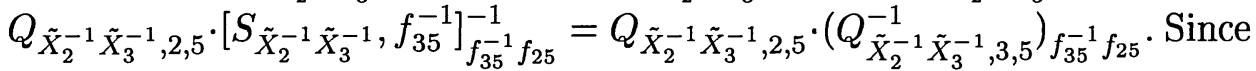
$\psi\left(\tilde{X}_{2}^{-1} \tilde{X}_{3}^{-1}\right)=\left(\begin{array}{ll}2 & 3\end{array}\right)(3 \quad 4)$, the images of $\{1,2\}$ under it are $\{1,4\}$. But $\{1,4\} \cap\{2,5\}=\emptyset$ and $\{1,4\} \cap\{3,5\}=\emptyset$. Thus, we get by (i) that

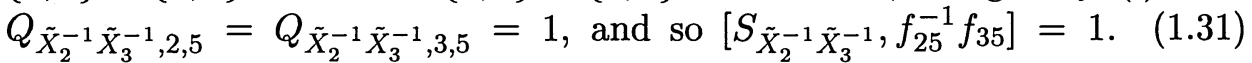
now implies $Q_{\tilde{X}_{2}^{-1}}=\left[S_{\tilde{X}_{2}^{-1}} \tilde{X}_{3}^{-1}, f_{13}^{-1}\right]$. By $(1.27) S_{\tilde{X}_{2}^{-1} \tilde{X}_{3}^{-1}}=S \cdot S_{\tilde{X}_{2}^{-1} \tilde{X}_{1}^{-1} \tilde{X}_{3}^{-1}}$ which gives

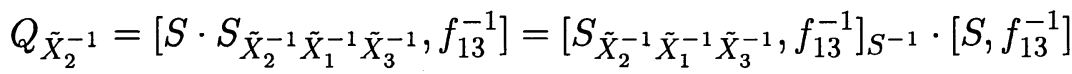

$$
\begin{aligned}
& =\left(Q_{\tilde{X}_{2}^{-1} \tilde{X}_{1}^{-2} \tilde{X}_{3}^{-1}, 1,3}\right)_{S^{-1}} \cdot Q \text {. }
\end{aligned}
$$

The value of $\psi\left(\tilde{X}_{2}^{-1} \tilde{X}_{1}^{-1} \tilde{X}_{3}^{-1}\right)\left(=\left(\begin{array}{lll}2 & 3\end{array}\right)\left(\begin{array}{ll}1 & 2\end{array}\right)\left(\begin{array}{ll}3 & 4\end{array}\right)\right)$ on $\{1,2\}$ is $\{2,4\}$. Since $\{2,4\} \cap\{1,3\}=\emptyset$ we get from part (i) of the Lemma that $Q_{\tilde{X}_{2}^{-1} \tilde{X}_{1}^{-1} \tilde{X}_{3}^{-1}, 1,3}=1$, therefore,

$$
Q_{\tilde{X}_{2}^{-1}}=Q \square \text { Lemma 2(ii) }
$$

Lemma 3. $\tau=Q^{-1}$.

Proof. By the assumption on $\tau, S_{\tilde{X}_{1}^{-1}}=S^{-1} \tau$. By definition of $T, T_{\tilde{X}_{1}^{-2}}=$ $S_{\tilde{X}_{2}^{-1} \tilde{X}_{1}^{-2}}$. We apply assumption $\left(1_{a}\right)$ twice to get, using $S_{\tilde{X}_{1}^{-1}}=S^{-1} \tau$, that 
$\left(S^{-1} S_{\tilde{X}_{2}^{-1}}\right)_{\tilde{X}_{1}^{-1}}=S_{\tilde{X}_{1}^{-1}}^{-1} S_{\tilde{X}_{2}^{-1}} \tilde{X}_{1}^{-1}=\tau^{-1} S \cdot S^{-1} S_{\tilde{X}_{2}^{-1}}=\tau^{-1} S_{\tilde{X}_{2}^{-1}}=\tau^{-1} T$.

Thus

$$
T_{\tilde{X}_{1}^{-2}}=\tau^{-1} T
$$

or

$$
\tau^{-1}=T_{\tilde{X}_{1}^{-2}} T^{-1}
$$

Applying $\tilde{X}_{1}^{2}$ on (1.32) and using $\tau_{\tilde{X}_{1}^{2}}=\tau$ (assumption $\left(2_{a}\right)$ ), we get

$$
\begin{aligned}
\tau^{-1} & =T \cdot T_{\tilde{X}_{1}^{2}}^{-1}=\left[T, \tilde{X}_{1}^{-2}\right]=\left[S_{\tilde{X}_{2}^{-1}}, f_{12}^{-1}\right]=\left[S,\left(f_{12}^{-1}\right)_{\tilde{X}_{2}}\right]_{\tilde{X}_{2}^{-1}}=\left[S, f_{13}^{-1}\right]_{\tilde{X}_{2}^{-1}} \\
& =Q_{\tilde{X}_{2}^{-1}} \stackrel{\text { by Lemma } 2}{=} Q
\end{aligned}
$$

that is,

(1.33) $\square$ Lemma 3

$$
\tau^{-1}=Q, \quad \text { or } \quad \tau=Q^{-1}
$$

Lemma 4. $\forall j \geq 3, \tau_{\tilde{X}_{j}}=\tau$.

Proof of Lemma 4. From $\tau=S S_{\tilde{X}_{1}^{-1}}$ and $S_{\tilde{X}_{j}}=S \forall j \geq 3$ it follows that $\tau_{\tilde{X}_{j}}=\tau \forall j \geq 3$.

Lemma 4

Lemma 5. $\tau_{\tilde{X}_{1}}=\tau$.

Proof of Lemma 5. Let us use now $\tau_{\tilde{X}_{1}}^{-1}=\tau_{T}\left(\left(2_{b}\right)\right.$ of the Proposition).

By Lemmas 2 and $3 \tau_{\tilde{X}_{2}}=\tau$.

Thus, $\tau_{T}=\tau_{S_{\tilde{X}_{2}^{-1}}}=S_{\tilde{X}_{2}^{-1}}^{-1} \tau S_{\tilde{X}_{2}^{-1}}=\left(S^{-1} \tau S\right)_{\tilde{X}_{2}^{-1}}=\left(\tau_{S}\right)_{\tilde{X}_{2}^{-1}}$.

So $\tau_{\tilde{X}_{1}^{-1}}=\tau_{\tilde{X}_{1}}=\tau_{T}^{-1}=\left(\tau_{S}^{-1}\right)_{\tilde{X}_{2}^{-1}}$, or

$$
\tau_{S}=\tau_{\tilde{X}_{1}^{-1} \tilde{X}_{2}}^{-1}
$$

Since $\tau_{\tilde{X}_{3}}=\tau$ and $S_{\tilde{X}_{3}}=S$, we get $\left(\tau_{S}\right)_{\tilde{X}_{3}}=\tau_{S}$ and

$$
\tau_{\tilde{X}_{1}^{-1} \tilde{X}_{2} \tilde{X}_{3}}=\left(\tau_{S}^{-1}\right)_{\tilde{X}_{3}}=\tau_{S}^{-1}=\tau_{\tilde{X}_{1}^{-1} \tilde{X}_{2}} .
$$

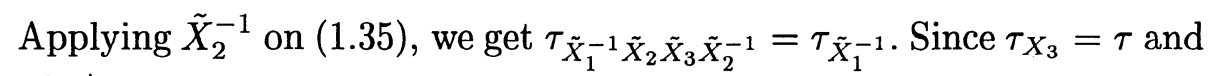
$\left\langle X_{2}, X_{3}\right\rangle=1, \tau_{\tilde{X}_{1}^{-1}} \tilde{X}_{2} \tilde{X}_{3} \tilde{X}_{2}^{-1}=\tau_{\tilde{X}_{1}^{-1}}^{-1} \tilde{X}_{3}^{-1} \tilde{X}_{2} \tilde{X}_{3} \stackrel{2}{=} \tau_{\tilde{X}_{3}^{-1}} \tilde{X}_{1}^{-1} \tilde{X}_{2} \tilde{X}_{3}=\tau_{\tilde{X}_{1}^{-1} \tilde{X}_{2} \tilde{X}_{3}}$. Thus:

$$
\tau_{\tilde{X}_{1}^{-1} \tilde{X}_{2} \tilde{X}_{3}}=\tau_{\tilde{X}_{1}^{-1}}
$$


Combining formulas (1.35)-(1.30) we get $\tau_{\tilde{X}_{1}^{-1}}=\tau_{\tilde{X}_{1}^{-1} \tilde{X}_{2}}$. Applying it to

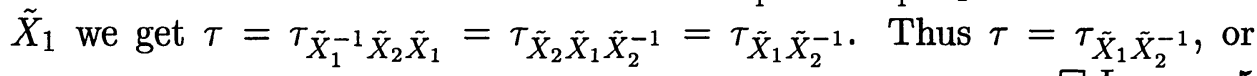
$\tau_{\tilde{X}_{1}}=\tau_{\tilde{X}_{2}}=\tau$.

Lemma 5

Lemma 6. $\tau_{\tilde{X}_{j}}=\tau \forall j=1,2, \ldots, n-1$.

Proof of Lemma 6. By Lemmas 2, 3, 4, 5.

Lemma 7. $\tau_{S}=\tau^{-1}$.

Proof of Lemma 7. From $\tau_{\tilde{X}_{1}}=\tau_{\tilde{X}_{2}}=\tau$ and (1.34).

Lemma 8. $\tau_{S}=\tau$.

Proof of Lemma 8. Consider assumption $\left(1_{b}\right)$ of the Proposition:

$$
S_{\tilde{X}_{1} \tilde{X}_{2}^{-1} \tilde{X}_{1}^{-1}}=S_{\tilde{X}_{1}}^{-1} S_{\tilde{X}_{1} \tilde{X}_{2}^{-1}}
$$

Using $S_{\tilde{X}_{1}^{-1}}=S^{-1} \tau$ and $\tau_{\tilde{X}_{1}}=\tau$, we get $S=S_{\tilde{X}_{1}}^{-1} \tau$, or $S_{\tilde{X}_{1}}^{-1}=S \tau^{-1}$, $S_{\tilde{X}_{1}}=\tau S^{-1}$. Assumption $\left(1_{b}\right)$ now gives (using $\tau_{\tilde{X}_{i}}=\tau \forall i=1, \ldots, n-1$ ):

$$
\tau S_{\tilde{X}_{2}^{-1} \tilde{X}_{1}^{-1}}^{-1}=S \tau^{-1} \cdot \tau S_{\tilde{X}_{2}^{-1}}^{-1}=S S_{\tilde{X}_{2}^{-1}}^{-1}=S T^{-1} .
$$

On the other hand, by $\left(1_{a}\right)$ and $(2), S_{\tilde{X}_{2}^{-1}} \tilde{X}_{1}^{-1}=S^{-1} T$. Thus

$$
\tau S_{X_{2}^{-1}}^{-1} X_{1}^{-1}=\tau T^{-1} S
$$

We compare the last 2 expressions to get $\tau T^{-1} S=S T^{-1}$, or

$$
\tau=S T^{-1} S^{-1} T, \quad \text { or } \quad T_{S^{-1}}^{-1}=\tau T^{-1} .
$$

By Lemmas 3 and 6

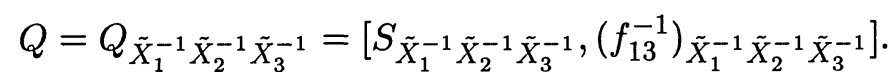

Thus:

$$
Q=\left[S_{\tilde{X}_{1}^{-1}} \tilde{X}_{2}^{-1} \tilde{X}_{3}^{-1}, f_{24}^{-1}\right]
$$




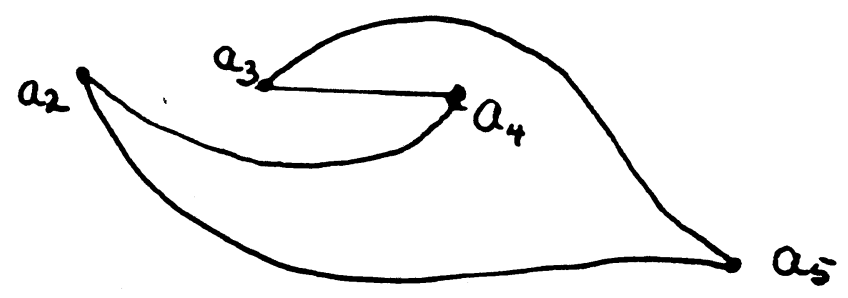

Figure III.7.2.

(we use $\left.(\{1,3\}) \psi\left(\tilde{X}_{1}^{-1} \tilde{X}_{2}^{-1} \tilde{X}_{3}^{-1}\right)=(\{1,3\})(1 \quad 2)(2 \quad 3)(3 \quad 4)=\{4,2\}\right)$.

Considering a quadrangle formed by $a_{2}, a_{3}, a_{4}, a_{5}$ (see Fig. III.7.2)

we can write in $\tilde{\tilde{P}}_{n}$ (Lemma III.2.2) $f_{35} f_{24}=f_{25} f_{34}$, or $f_{24}=f_{35}^{-1} f_{25} f_{34}$, $f_{24}^{-1}=f_{34}^{-1} f_{25}^{-1} f_{35}$. From (1.38) we get, denoting by $b=\tilde{X}_{1}^{-1} \tilde{X}_{2}^{-1} \tilde{X}_{3}^{-1}$,

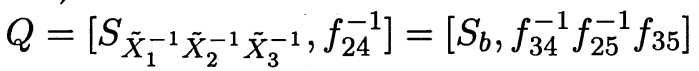

$$
\begin{aligned}
& =\left[S_{b}, f_{34}^{-1}\right]\left[S_{b}, f_{25}^{-1} f_{35}\right]_{f_{34}}=Q_{b, 3,4} \cdot\left[S_{b}, f_{25}^{-1}\right]_{f_{34}} \cdot\left[S_{b}, f_{35}\right]_{f_{25} f_{34}} \\
& =Q_{b, 3,4} \cdot\left(Q_{b, 2,5}\right)_{f_{34}} \cdot\left[S_{b}, f_{35}^{-1}\right]_{f_{35}^{-1} f_{25} f_{34}}^{-1}=Q_{b, 3,4} \cdot\left(Q_{b, 2,5}\right)_{f_{34}} \cdot\left(Q_{b, 3,5}\right)_{X}^{-1} .
\end{aligned}
$$

Now, $(\{1,2\}) \psi(b)=\{1,2\}(1 \quad 2)(2 \quad 3)(3 \quad 4)=\{4,1\}$. Since $\{4,1\} \cap$ $\{2,5\}=\emptyset$ and $\{4,1\} \cap\{3,5\}=\emptyset$, we get by Lemma 2 that $Q_{b, 2,5}=Q_{b, 3,5}=1$, and by (1.39)

$$
Q=Q_{b, 3,4}
$$

We can write:

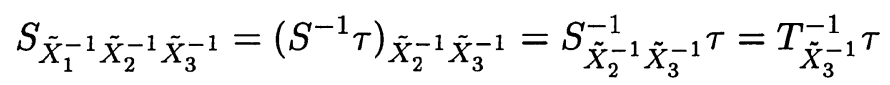

(using $\tau_{\tilde{X}_{i}}=\tau \forall i$ ). So

$$
\begin{aligned}
& Q=Q_{b, 3,4}=\left[S_{\tilde{X}_{1}^{-1}} \tilde{X}_{2}^{-1} \tilde{X}_{3}^{-1}, f_{34}^{-1}\right] \stackrel{\text { by } f_{34}=\tilde{X}_{3}^{2}}{=}\left[T_{\tilde{X}_{3}^{-1}}^{-1} \tau, \tilde{X}_{3}^{-2}\right] \\
& =\left[\tau, \tilde{X}_{3}^{-2}\right] T_{\tilde{X}_{3}^{-1}} \cdot\left[T_{\tilde{X}_{3}^{-1}}^{-1}, \tilde{X}_{3}^{-2}\right]=\left[T_{\tilde{X}_{3}^{-1}}^{-1}, \tilde{X}_{3}^{-2}\right]=\left[T^{-1}, \tilde{X}_{3}^{-2}\right]_{\tilde{X}_{3}^{-1}} .
\end{aligned}
$$

Since $Q_{\tilde{X}_{3}}=Q$, we get

$$
Q=\left[T^{-1}, \tilde{X}_{3}^{-2}\right] .
$$


This implies

$$
\begin{aligned}
Q_{S^{-1}}= & \left(T^{-1} T_{\tilde{X}_{3}^{2}}\right)_{S^{-1}} \stackrel{\text { assumption } 3}{=} T_{S^{-1}}^{-1} \cdot\left(T_{S^{-1}}\right)_{\tilde{X}_{3}^{2}} \\
& =\left[T_{S^{-1}}^{-1}, \tilde{X}_{3}^{-2}\right] \stackrel{\text { by }}{\stackrel{(1.37)}{=}}\left[\tau T^{-1}, \tilde{X}_{3}^{-2}\right] \\
& =\left[T^{-1}, \tilde{X}_{3}^{-2}\right]_{\tau^{-1}}\left[\tau, \tilde{X}_{3}^{-2}\right] \stackrel{\text { by Lemma } 4}{=}\left[T^{-1}, \tilde{X}_{3}^{-2}\right]_{\tau^{-1}} \stackrel{\text { by } \stackrel{(1.40)}{=} Q_{\tau^{-1}} .}{ }
\end{aligned}
$$

Using $Q=\tau^{-1}$ we get $\tau_{S^{-1}}^{-1}=\tau_{\tau^{-1}}^{-1}$. Thus, $\tau_{S^{-1}}=\tau$ and $\tau_{S}=\tau$. Lemma 8

We can now finish the proof of Proposition III.7.1.

By Lemma III.2.4, we only have to prove that $\tau^{2}=1, \tau_{b}=\tau \forall b \in \tilde{\tilde{B}}_{n}$ and $\tau \in \operatorname{Center}(G)$.

By the previous Lemma, $\tau_{S}=\tau$, and by Lemma $7, \tau_{S}=\tau^{-1}$. Thus, $\tau=\tau^{-1}$ and $\tau^{2}=1$.

By Lemma $6, \tau_{\tilde{X}_{i}}=\tau \forall i \in(1, \ldots, n-1)$. Thus $\tau_{b}=\tau \forall b \in \tilde{\tilde{B}}_{n}$.

By Lemma $8 \tau_{S}=\tau$, i.e. $[\tau, S]=1$. Let $b \in \tilde{B}_{n}:\left[\tau, S_{b}\right]=\left[\tau_{b^{-1}}, S\right]_{b}=$ $[\tau, S]_{b}=1$.

Thus $\tau$ commutes with $S_{b} \forall b \in \tilde{\tilde{B}}_{n}$. Since $\operatorname{ker}\left(\tilde{B}_{n} \rightarrow \tilde{\tilde{B}}_{n}\right)$ acts trivially on $G, \tilde{\tilde{B}}_{n}$ acts on $G$ via $\tilde{B}_{n}$, and thus $\tau$ commutes with $S_{b} \forall b \in \tilde{B}_{n}$.

By assumption (0) of the proposition, $G$ is generated by $\left\{S_{b}\right\}_{b \in \tilde{B}_{n}}$. Thus $\tau \in \operatorname{Center}(G)$.

$\square$ Proposition III.7.1

\section{New Set of Generators for $G$.}

Recall from Chapter II that $G=G\left(\varepsilon_{18}\right)=\pi_{1}\left(\mathbb{C P}^{2}-S_{3}, *\right)$ is generated by $E_{i}, E_{i}^{\prime}$ and satisfies the relations listed in Theorem II.6.

In this chapter we shall introduce new generators for $G$, using the braid group $B_{9}$ and the quotient $\tilde{B}_{9}$ from Chapter III.

\section{IV.1. New presentation of $B_{9}$.}

Definition. $T_{i} \quad i=1, \ldots, 9 \quad i \neq 4$

Consider a geometric model $(D, k)$ for $\# K=9$ as in Fig. IV.1.1.

Let $\left\{t_{i}\right\}_{i=1 \quad 04}^{0}$ be paths connecting different parts of $K$ as in Fig. IV.1.2.

Let $T_{i}$ be the half-twist corresponding to $t_{i} \quad i=1, \ldots, 9 i \neq 4 .\left(T_{i}=\right.$ $\left.H\left(t_{i}\right)\right)$ 


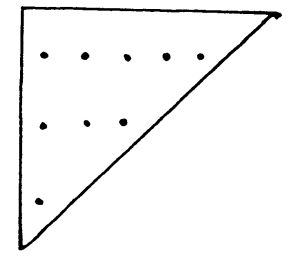

Figure IV.1.1

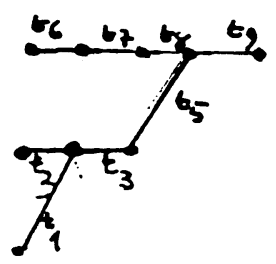

Fig. IV.1.2.

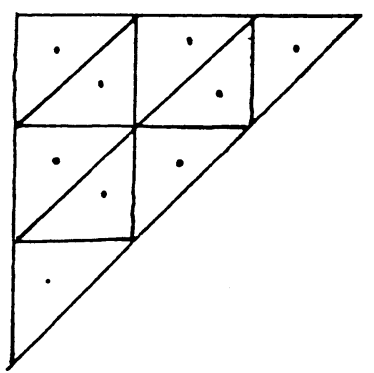

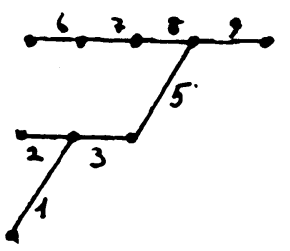

Figure IV.1.3.

Lemma IV.1.0. $T_{i}$ and $T_{j}$ are adjacent for $(i, j)$ as follows:

$$
\begin{array}{ll}
i, j \in\{1,2,3\} & \\
i=5 & j=3,8,9 \\
i=6,7,8 & j=i+1 .
\end{array}
$$

$T_{i}$ and $T_{j}$ are disjoint for $(i, j)$ as follows:

$$
\begin{array}{ll}
i \in\{1,2,3\} & j \in\{6,7,8,9\} \\
i=5 & j=1,2,6,7 \\
i=6 & j=8,9 \\
i=7 & j=9 .
\end{array}
$$

Proof. From Fig. IV.1.2.

Remark. The choice of the model comes from the configuration of planes in the degeneration of $V_{3}$ to the union of planes as in Fig.II.1. (We constructed this degeneration in BGT III [MoTe7].) In each of the triangles we choose a point (Fig. IV.1.3).

We choose a path connecting 2 points in neighboring triangles as in Fig. IV.1.4.

We then get a configuration which is basically equal to the one in Fig. IV.1.2. 


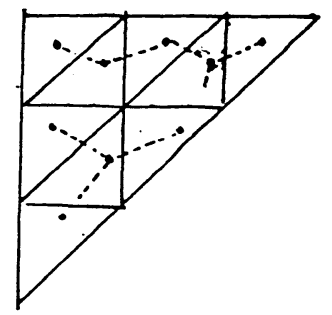

Figure IV.1.4.

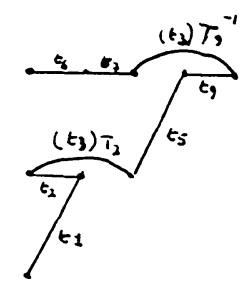

Figure IV.1.5.

Since we do not need all possible connections to get a set of generators for $B_{9}$, we skip the connection between the points in $P_{3}$ and $P_{5}$.

Lemma IV.1.1. There exists a presentation of the braid group $B_{9}$, as follows:

$$
B_{9}=\left\langle T_{i .} \mid i=1, \ldots, 9, \quad i \neq 4\right\rangle
$$

and the following is a complete set of relations:

$$
\begin{aligned}
& \left\langle T_{i}, T_{j}\right\rangle=1 \quad \text { if } T_{i} \text { and } T_{j} \text { are consecutive } \\
& {\left[T_{i}, T_{j}\right]=1 \quad \text { if } T_{i} \text { and } T_{j} \text { are disjoint }} \\
& {\left[T_{1}, T_{2}^{-1} T_{3} T_{2}\right]=1} \\
& {\left[T_{5}, T_{8}^{-1} T_{9} T_{8}\right]=1 .}
\end{aligned}
$$

Proof. Consider the geometric model $(D, K), \# K=9$ as in Fig. IV.1.1. We choose a frame in $B_{9}[D, K]$ where each half-twist in the frame corresponds to a path, as in Fig. IV.1.5.

In terms of $T_{i}$, this frame is

$$
T_{1}, T_{2}, T_{2}^{-1} T_{3} T_{2}, T_{5}, T_{9}, T_{9} T_{8} T_{9}^{-1}, T_{7}, T_{6}
$$

By E. Artin's presentation of the braid group (see Chapter 0), we know that $B_{9}$ is generated by the above frame and the only relations are triple 


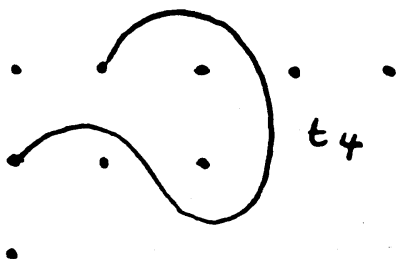

Figure IV.1.6.

relations for non-neighboring elements and commutation relations for neighboring elements. Thus, a full set of relations is:

$$
\begin{aligned}
\left\langle T_{1}, T_{2}\right\rangle & =\left\langle T_{2}, T_{2}^{-1} T_{3} T_{2}\right\rangle=\left\langle T_{2}^{-1} T_{3} T_{2}, T_{5}\right\rangle=\left\langle T_{5}, T_{9}\right\rangle \\
& =\left\langle T_{9}, T_{9} T_{8} T_{9}^{-1}\right\rangle=\left\langle T_{9} T_{8} T_{9}^{-1}, T_{7}\right\rangle=\left\langle T_{7}, T_{6}\right\rangle=1
\end{aligned}
$$

and all possible commutation relations between other elements of the frame.

Since $T_{3}=T_{2}\left(T_{2}^{-1} T_{3} T_{2}\right) T_{2}^{-1}$ and $T_{9}=T_{9}^{-1}\left(T_{9} T_{8} T_{9}^{-1}\right) T_{9}$, then $T_{1}, T_{2}, T_{3}, T_{5} T_{9}, T_{8}, T_{7}, T_{6}$ generates $B_{9}$. Translating the above relations to these generators and using simple facts about commutators, we obtain the Lemma.

Definition. $T_{4}$.

$T_{4}=T_{2}^{-1} T_{3} T_{7}^{-1} T_{8} T_{5} T_{8}^{-1} T_{7} T_{3}^{-1} T_{2}$. It is possible to notice that $T_{4}$ is the half-twist that corresponds to the path $t_{4}$ as in Fig. IV.1.6, and thus $T_{4}$ is adjacent to $T_{2}$ and $T_{6}$, transversal to $T_{7}$ and $T_{3}$ and disjoint from the others.

\section{IV.2. Presentation of $\tilde{\mathbf{B}}_{\mathbf{9}}$.}

Let $\tilde{B}_{9}$ be as in Chapter III, $\tilde{B}_{9}=B_{9} / T$, where $T=\langle[X, Y]\rangle$, and $X, Y$ are transversal.

Let $T_{i}$ be as in $\S 1$. Let $\tilde{T}_{i}$ be the images of $T_{i}$ in $\tilde{B}_{9}$.

Lemma IV.2.1. $\tilde{B}_{9}$ is generated by $\left\langle\tilde{T}_{i} \mid i=1 \ldots 9\right\rangle$ and the only relations are:

(1) $\left\langle\tilde{T}_{i}, \tilde{T}_{j}\right\rangle=1 \quad T_{i}, T_{j}$ are consecutive $i, j \neq 4$

(2) $\left[\tilde{T}_{i}, \tilde{T}_{j}\right]=1 \quad T_{i}, T_{j}$ are disjoint $i, j \neq 4$

(3) $\left[\tilde{T}_{1}, \tilde{T}_{2}^{-1} \tilde{T}_{3} \tilde{T}_{2}\right]=1$ 
(4) $\left[\tilde{T}_{5}, \tilde{T}_{8}^{-1} \tilde{T}_{9} \tilde{T}_{8}\right]=1$

(5) $\quad \tilde{T}_{4}=\tilde{T}_{2}^{-1} \tilde{T}_{3} \tilde{T}_{7}^{-1} \tilde{T}_{8} \tilde{T}_{5} \tilde{T}_{8}^{-1} \tilde{T}_{7} \tilde{T}_{3}^{-1} \tilde{T}_{2}$

Proof. By the previous Lemma, $\tilde{B}_{9}$ is generated by $\tilde{T}_{i}, i \neq 4$ with a full set of relations (1)... (4). We add one generator and express it in terms of the other relations to get $\tilde{T}_{4}$ and relation (5).

Lemma IV.2.2. (1) $\left\langle\tilde{T}_{4}, \tilde{T}_{2}\right\rangle=1$.

(2) $\left\langle\tilde{T}_{4}, \tilde{T}_{6}\right\rangle=1$.

(3) $\left\langle\tilde{T}_{7}, \tilde{T}_{6}\right\rangle=1$.

(4) $\left[\tilde{T}_{4}, \tilde{T}_{i}\right]=1 \quad i=1,3,5,9,8$.

Proof. We use Theorem III.3.1 from BGT I.

Since $T_{4}$ is consecutive to $T_{i}, \quad i=2,6$ and $T_{6}$ is consecutive to $T_{7}$ then $\left\langle T_{4}, T_{2}\right\rangle=\left\langle T_{4}, T_{6}\right\rangle=\left\langle T_{6}, T_{7}\right\rangle=1$. Thus, $\left\langle\tilde{T}_{4}, \tilde{T}_{2}\right\rangle=\left\langle T_{4}, T_{6}\right\rangle=\left\langle\tilde{T}_{6}, \tilde{T}_{7}\right\rangle=1$.

$T_{4}$ and $T_{i}$ for $i=1,9$ are disjoint, therefore, $\left[T_{4}, T_{i}\right]=1$ for $i=1,9$. Thus, $\left[\tilde{T}_{4}, \tilde{T}_{i}\right]=1$ for $i=1,9$. The half-twists $T_{4}$ and $T_{i} \quad i=3,5,8$ are transversal and, thus, $\left[\tilde{T}_{4}, \tilde{T}_{i}\right]=1$ for $i=3,5,8$ (Remark III.1.1).

We need the following relations of $\tilde{T}_{i}$ in order to get a smaller set of generators for $G$.

\section{Lemma IV.2.3.}

$$
\begin{array}{ll}
\left(\tilde{T}_{4}\right)_{\tilde{T}_{2}^{-1}} \tilde{T}_{3} \tilde{T}_{7}^{-1} \tilde{T}_{8}=\tilde{T}_{5} & \text { preserving polarization } \\
\left(\tilde{T}_{2}\right)_{\tilde{T}_{4}} \tilde{T}_{3} \tilde{T}_{5}^{-1} \tilde{T}_{7}^{-1}=\tilde{T}_{8} & \text { preserving polarization } \\
\left(\tilde{T}_{3}\right)_{\tilde{T}_{2}^{-1}} \tilde{T}_{4} \tilde{T}_{5}^{-1} \tilde{T}_{8}=\tilde{T}_{7} & \text { preserving polarization }
\end{array}
$$

Proof. It is actually true for $T_{i}$ instead of $\tilde{T}_{i}$. It can be verified geometrically using Fig. III.1.1 for a geometric presentation of a half-twist conjugated by another half-twist.

Lemma IV.2.4. Another presentation of $\tilde{B}_{9}$.

Let $\tilde{T}_{1^{\prime}}=\tilde{T}_{2}^{+2} \tilde{T}_{1} \tilde{T}_{2}^{-2} \quad \tilde{T}_{i}^{\prime}=\tilde{T}_{i} \quad i \neq 1$. Then $\tilde{B}_{9}$ is generated by $\tilde{T}_{i}^{\prime}$ and the following is a complete set of relations: 
(1) $\left\langle\tilde{T}_{i}^{\prime}, \tilde{T}_{j}^{\prime}\right\rangle=1$ if $\tilde{T}_{i}, \tilde{T}_{j}$ are adjacent $i, j \neq 4$.

(2) $\left[\tilde{T}_{i}^{\prime}, \tilde{T}_{j}^{\prime}\right]=1$ if $\tilde{T}_{i}, \tilde{T}_{j}$ are disjoint $i, j \neq 4$.

(3) $\left[\tilde{T}_{1}^{\prime}, \tilde{T}_{2}^{\prime} \tilde{T}_{3}^{\prime} \tilde{T}_{2}^{\prime-1}\right]=1$

(4) $\left[\tilde{T}_{5}^{\prime}, \tilde{T}_{8}^{\prime-1} \tilde{T}_{9}^{\prime} \tilde{T}_{8}\right]=1$

(5) $\tilde{T}_{4}^{\prime}=\tilde{T}_{2}^{\prime-1} \tilde{T}_{3}^{\prime} \tilde{T}_{7}^{\prime-1} \tilde{T}_{8}^{\prime} \tilde{T}_{5}^{\prime} \tilde{T}_{8}^{\prime-1} \tilde{T}_{7}^{\prime} \tilde{T}_{3}^{\prime-1} \tilde{T}_{2}^{\prime}$

Proof. Clearly, $T_{i}=T_{i}^{\prime} i \neq 1 \quad T_{1}=\tilde{T}_{2}^{\prime-2} \tilde{T}_{1}^{\prime} \tilde{T}_{2}^{\prime 2}$. We substitute these expressions in the relations of Lemma IV.2.1 to prove the Lemma.

$$
\text { IV.3. } \alpha: \tilde{\mathbf{B}}_{\mathbf{9}} \rightarrow \mathbf{G} \text {. }
$$

We want to prove that there exists $\alpha: \tilde{B}_{9} \rightarrow G$ s.t. $\alpha\left(\tilde{T}_{i}\right)=E_{i}$, for $E_{i}$ that were introduced in Chapter II. For that we prove certain relations that $E_{i}$ satisfy, based on Proposition II.6.

Lemma IV.3.1. (1) $\left\langle E_{i}, E_{j}\right\rangle=1$ if $T_{i}, T_{j}$ are adjacent $i, j \neq 4$.

(2) $\left[E_{i}, E_{j}\right]=1$ if $T_{i}, T_{j}$ are disjoint $i, j \neq 4$.

(3) $\left[E_{1}, E_{2} E_{3} E_{2}^{-1}\right]=1$

(4) $\left[E_{9}, E_{5}^{-1} E_{8} E_{5}\right]=1$

(5) $E_{4}=E_{2}^{-1} E_{3} E_{7}^{-1} E_{8} E_{5} E_{8}^{-1} E_{7} E_{3}^{-1} E_{2}$.

Proof. We use Proposition II.6, which states a list of relations satisfied by the $E_{i}$.

(1), (2) By Fig. IV.1.3, $T_{i}, T_{j} \quad(i, j \neq 4)$ are adjacent, $\Leftrightarrow \hat{L}_{i}$ and $\hat{L}_{j}$ are edges of some triangle, $\Leftrightarrow$ (by Corollary II.3) $\psi\left(E_{i}\right)$ and $\psi\left(E_{j}\right)$ have one common index. Moreover, $T_{i}$ and $T_{j}$ are disjoint $\Leftrightarrow \psi\left(E_{i}\right)$ and $\psi\left(E_{j}\right)$ are disjoint. By (1) and (2) of Proposition II.6 we get (1) and (2) of this Proposition.

(3) By Proposition II.6 (3), $E_{8}=E_{7} E_{5} E_{3}^{-1} E_{4}^{-1} E_{2} E_{4} E_{3} E_{5}^{-1} E_{7}^{-1}$. By Proposition II.6 (2), $\left[E_{3}, E_{4}\right]=1$. Thus, $E_{8}=E_{7} E_{5} E_{4}^{-1} E_{3}^{-1} E_{2} E_{3} E_{4}$ $E_{5}^{-1} E_{7}^{-1}$. By Proposition II.6 (1), $E_{3}^{-1} E_{2} E_{3}=E_{2} E_{3} E_{2}^{-1}$. Thus, 

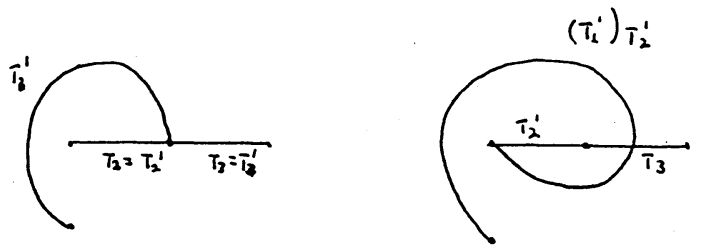

Figure IV.3.1.

$E_{2} E_{3} E_{2}^{-1}=E_{4} E_{5}^{-1} E_{7}^{-1} E_{8} E_{7} E_{5} E_{4}^{-1}$. By Proposition II.6 (2) $E_{i}$, for $i=4,5,7,8$, commutes with $E_{1}$. Thus, $\left[E_{1}, E_{2} E_{3} E_{2}^{-1}\right]=1$.

(4) Вy (3), $E_{8}=E_{7} E_{5} E_{3}^{-1} E_{4}^{-1} E_{2} E_{1} E_{3} E_{5}^{-1} E_{7}^{-1}$. By (2), $\left[E_{5}, E_{7}\right]=1$. newline Thus, $E_{5}^{-1} E_{8} E_{5}=E_{7} E_{3}^{-1} E_{4}^{-1} E_{2} E_{4} E_{3} E_{7}^{-1}$.

For $i=7,3,4,2, E_{i}$ commutes with $E_{9}$. Thus, $\left[E_{9}, E_{5}^{-1} E_{8} E_{5}\right]=1$.

(5) By Proposition II.6 (3), $E_{4}^{-1} E_{2} E_{4}=E_{3} E_{5}^{-1} E_{7}^{-1} E_{8} E_{7} E_{5} E_{3}^{-1}$. By Proposition II.6 (2)(1), $E_{4}^{-1} E_{2} E_{4}=E_{2} E_{4} E_{2}^{-1}$ and $\left[E_{5}, E_{7}\right]=1$. By Proposition II.6 (1) $E_{5}^{-1} E_{8} E_{5}=E_{8} E_{5} E_{8}^{-1}$. Thus, $E_{2} E_{4} E_{2}^{-1}=$ $E_{3} E_{7}^{-1} E_{5}^{-1} E_{8} E_{5} E_{7} E_{3}^{-1} . E_{4}=E_{2}^{-1} E_{3} E_{7}^{-1} E_{8} E_{5} E_{8}^{-1} E_{7} E_{3}^{-1} E_{2}$.

We first prove that there exist $\alpha^{\prime}: \tilde{B}_{9} \rightarrow G$ such that $\alpha^{\prime}\left(\tilde{T}_{i}^{\prime}\right)=E_{i}$.

Lemma IV.3.2. There exists a homomorphism $\alpha^{\prime}, \alpha^{\prime}: \tilde{B}_{9} \rightarrow G$ such that $\alpha^{\prime}\left(\tilde{T}_{i}^{\prime}\right)=E_{i}$.

Proof. By Lemma IV.2.4, $\tilde{B}_{9}$ is generated by $\tilde{T}_{i}^{\prime}$. Thus, we define $\alpha^{\prime}\left(\tilde{T}_{i}^{\prime}\right)=$ $E_{i}$. To prove that $\alpha^{\prime}$ induces a homomorphism, we have to show that $E_{i}$ satisfies any relation that $\tilde{T}_{i}^{\prime}$ satisfies. In IV.2.4 we presented a full list of relations for $\tilde{T}_{i}^{\prime}$. In Lemma IV.3.1 we proved that these relations are satisfied when $\tilde{T}_{i}^{\prime}$ is replaced by $E_{i}$.

\section{Lemma IV.3.3.}

$$
\left[E_{1}, E_{2}^{-1} E_{3} E_{2}\right]=1
$$

Proof. $T_{3}^{\prime}$ is transversal to $T_{2}^{\prime} T_{1}^{\prime} T_{2}^{-1}$. (See Fig. IV.3.1)

Thus, $\left[\tilde{T}_{2}^{\prime} \tilde{T}_{1}^{\prime} \tilde{T}_{2}^{-1}, \tilde{T}_{3}^{\prime}\right]=1$.

Thus, $\alpha^{\prime}\left[\tilde{T}_{2}^{\prime} \tilde{T}_{1}^{\prime} \tilde{T}_{2}^{-1}, \tilde{T}_{3}^{\prime}\right]=1$. 
Thus, $\left[E_{2} E_{1} E_{2}^{-1}, E_{3}\right]=1$.

Thus, $\left[E_{1}, E_{2}^{-1} E_{3} E_{2}\right]=1$.

Lemma IV.3.4. There exists $\alpha: \tilde{B}_{9} \rightarrow G$ such that $\alpha\left(\tilde{T}_{i}\right)=E_{i}$.

Proof. We use the presentation of $\tilde{B}_{9}$ from Lemma IV.2.1 where $\tilde{B}_{9}$ is generated by $\tilde{T}_{i}$. We define $\alpha\left(\tilde{T}_{i}\right)=E_{i}$. The relations listed in IV.2.1 are satisfied when $\tilde{T}_{i}$ is replaced by $E_{i}$ by Lemma IV.3.1 and Lemma IV.3.3. Thus we can extend the definition of $\alpha$ to the whole of $\tilde{B}_{9}$ in a natural way.

\section{IV.4. Prime elements in $B_{9}$.}

We now recall a few results from Chapters II and III concerning the braid group $B_{n}$ and the quotient group $\tilde{B}_{n}$. We refer the reader to Chapter III for the definition of prime element with s.h.t. (supporting half-twist) $T$, and central element $c$.

We quote here a few results from [MoTe4], Chapter II and Chapter III.

Lemma IV.4.0. If $X$ and $Y$ are 2 consecutive half-twists in $B_{n}$, then

(a) $X Y X=Y X Y$.

(b) $X Y X^{-1}=Y^{-1} X Y$.

(c) $(Y)_{X^{-1}}=(X)_{Y}$.

(d) $(Y)_{X^{-1}}$ is consecutive to $X$ and to $Y$. It is the half-twist corresponding to a path connecting those ends of $X$ and $Y$ which are not a common index of $X$ and $Y$.

(e) $u=\tilde{X}_{\tilde{Y}^{-1}}^{2} \tilde{Y}^{-2}=\tilde{Y}_{\tilde{X}}^{2} \cdot \tilde{Y}^{-2}$, is an element of $\tilde{P}_{n} ; u$ is a prime element with s.h.t. $\tilde{X}$ and central element $c=\left[\tilde{Y}^{2}, \tilde{X}^{2}\right]$ (i.e., $c^{2}=1, c \in$ $\left.\operatorname{Center}\left(\tilde{P}_{n}\right)\right)$.

(f) $\left[\tilde{X}^{\prime 2}, \tilde{Y}^{\prime 2}\right]=\left[\tilde{X}^{\prime 2}, \tilde{Y}^{-2}\right]=\left[\tilde{X}^{\prime-2}, \tilde{Y}^{\prime-2}\right]=c \forall X^{\prime}, Y^{\prime}$ a pair of consecutive half-twists where $c^{2}=1, c \in \operatorname{Center}(G)$.

(g) $\tilde{Y}^{-2}\left(\tilde{Y}^{2}\right)_{\tilde{X}^{-1}}=c$ (inverse of a prime element of $\tilde{P}_{n}$ with s.h.t. $\left.X\right)$. 


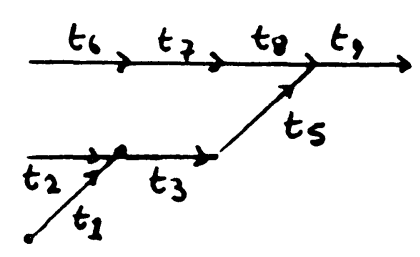

Figure IV.4.1.

(h) If $Z$ is transversal to $X, g$ a prime element with s.h.t. $X$, then $(g)_{Z}=$ $g$.

Proof.

(a) From Lemma III.3.1 [MoTe4].

(b) From Lemma II.4 and (a).

(c) From (b).

(d) Let $Y=H(y), y$ connects $a$ and $b$ and $X=H(x) x$ connects $b$ and c. Assume $X$ corresponds to a diffeomorphism $\beta: D \rightarrow D$ s.t. $\beta(b)=c$. Then $(Y)_{X^{-1}}=H\left((\sigma) \beta^{-1}\right)$. Clearly, $(\sigma) \beta^{-1}$ is a path connecting $a$ and $c$. (See Claim III.1.0 and Fig. III.1.1.)

(e) Lemma III.6.1.

(f) Proposition III.5.2.

(g) We shall prove that $c\left(Y^{-2}\left(Y^{2}\right)_{X^{-1}}\right)^{-1}$ is a prime element:

$$
c\left(Y^{-2}\left(Y^{2}\right)_{X^{-1}}\right)^{-1}=c\left(Y^{-2}\right)_{X^{-1}} Y^{2}=Y^{2}\left(Y^{-2}\right)_{X^{-1}} \quad \text { (by (b) ). }
$$

Let $T=(Y)_{X^{-1}}$. By (e) and (c), $(T)_{X}^{2} \cdot T^{-2}$ is a prime element. But $(T)_{X}=\left((Y)_{X^{-1}}\right)_{X}=Y$. Thus $Y^{2}(Y)_{X^{-1}}^{-2}$ is a prime element.

(h) Lemma III.4.3.

\section{$\xi$-situation:}

Consider $\tilde{B}_{9}, \tilde{P}_{9}$ as $\tilde{B}_{9}$-groups by conjugation.

Let $\tilde{T}_{1}, \ldots, \tilde{T}_{9}$ be as in $\S 1$.

We choose a polarization on $\tilde{T}_{i}$ from smaller end index to bigger end index as shown in Fig. IV.4.1.

Let $\xi_{1}=\left(\tilde{T}_{2}\right)_{\tilde{T}_{1}}^{2}\left(\tilde{T}_{2}\right)^{-2}$. 
By Lemma IV.4.0 (e), $\xi_{1}$ is a prime element in $\tilde{P}_{9}$ with s.h.t. $\tilde{T}_{1}$ and central element $\left[T_{2}^{2}, T_{1}^{2}\right]$.

Let $c$ be the corresponding central element. Thus, $c \in \operatorname{Center}\left(\tilde{P}_{9}\right), \quad c^{2}=$ $1, c=\left[T_{2}^{2}, T_{1}^{2}\right]$.

Let $\xi_{i}$ be the unique prime element in $\tilde{P}_{9}$ s.t. $\left(\xi_{i}, \tilde{T}_{i}\right)$ is coherent with $\left(\xi_{1}, \tilde{T}_{1}\right) i=2, \ldots, 9$. (See Proposition III.3.3).

Claim IV.4.1. (1) $c$ is the corresponding central element of $\left(\xi_{i}, T_{i}\right) \quad \forall i=$ $1 \ldots 9, c^{2}=1$.

(2) $c \in \operatorname{Center}\left(\tilde{B}_{9}\right)$.

(3) $\xi_{i}=(Y)_{\tilde{T}_{i}}^{2} Y^{-2}$ for some $Y$ half-twist adjacent to $T_{i}$.

(4) $c=\left[\tilde{T}_{k}^{2}, \tilde{T}_{\ell}^{2}\right] \quad \forall k, \ell$ s.t. $T_{k}$ and $T_{\ell}$ are consecutive.

(5) $\xi_{i}$ is a prime element of $\tilde{B}_{9}$.

(6) Let $X, Y$, be 2 half-twists, $X=H(x), \quad Y=H(y), \quad T_{i}=H\left(t_{i}\right)$ s.t. $x, y, t_{i}$ make a triangle. Assume that $x$ and $y$ meet in $v$, and a counter clockwise rotation around $v$ inside the triangle meets $x$ before it meets y (Fig. IV.4.2(a) and (b)). Then $\tilde{T}_{i}$, the s.h.t. of $\xi_{i}$ satisfies $T_{i}=X Y X^{-1}$. And: (i) If the polarization of $T_{i}$ goes from $x$ to $y$, then $\xi_{i}=\tilde{X}^{2} \tilde{Y}^{-2}$. (ii) If the polarization of $T_{i}$ goes from $y$ to $x$, then $\xi_{i}=X^{-2} Y^{2}$.

Proof.

(1) The pair $\left(\xi_{i}, \tilde{T}_{i}\right)$ is coherent with $\left(\xi_{1}, \tilde{T}_{1}\right)$. Thus, $\xi_{i}$ is conjugate to $T_{1}$ and $\tilde{T}_{i}$ is conjugate to $f_{1}$ by some $B_{i}$. Then by Lemma III.3.1, their corresponding central element is equal.

(2) A priori, $c \in \operatorname{Center}\left(\tilde{P}_{9}\right)$. We have to prove that $c \in \operatorname{Center}\left(\tilde{B}_{9}\right)$. Consider $\tilde{P}_{9}$ as $\tilde{B}_{9}$-group. $\xi_{i}$ is a prime element in $\tilde{P}_{9}$ as a a $\tilde{B}_{9}$-group where $c$ is the central element of $\left(\xi_{i}, T_{i}\right)$. Thus, we have $(c) b=c \quad \forall b \in$ $\tilde{B}_{9}$. (( $\left.\quad\right) b=$ action of $\tilde{B}_{9}$ on $\left.\tilde{P}_{9}\right)$, but $(c) b=c_{b}$ by definition. Thus, $c_{b}=c \quad \forall b \in \tilde{B}_{9} \Rightarrow c \in \operatorname{Center}\left(\tilde{B}_{9}\right)$.

(3) The pair $\left(\xi_{i}, T_{i}\right)$ is coherent with the pair $\left(\xi_{1}, \tilde{T}_{1}\right)$. Thus, $\exists b_{i} \in B$ s.t. $\xi_{i}=\left(\xi_{1}\right)_{b_{i}}$ and $\tilde{T}_{i}=\left(\tilde{T}_{1}\right)_{b_{i}}$. Denote $\left(\tilde{T}_{2}\right)_{b_{i}}=\tilde{Y}$ and apply conjugation by $b_{i}$ on $\xi_{1}=\left(\tilde{T}_{2}^{2}\right)_{T_{1}} \tilde{T}_{2}^{-2}$. 

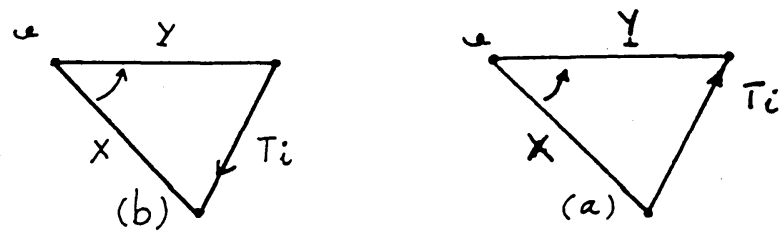

Figure IV.4.2.

(4) By Proposition III.5.2.

(5) By construction, $\xi_{i}$ is a prime element of $\tilde{P}_{9}$ with central element $c$. In (2) we proved that $c \in \operatorname{Center}\left(\tilde{B}_{9}\right)$. Thus, $\xi_{i}$ is also a prime element of $\tilde{B}_{9}$.

(6) Consider the two triangles (Fig. IV.4.2(a) and (b)):

If the polarization of $T_{i}$ goes from $X$ to $Y$ as in Fig. (a) (from $Y$ to $X$ as in Fig. (b)) then we take $b \in \tilde{B}_{n}$ s.t. $\left(T_{1}\right) b=\tilde{T}_{i}\left(T_{2}\right) b=Y$ preserving polarization of $T_{1}$ (reversing polarization), respectively. Clearly, $\left(\left(T_{2}\right)_{T_{1}}\right) b=X$. Consider the polarized pair $\left(\xi_{1}, \tilde{T}_{1}\right)=\left(\left(\tilde{T}_{2}\right)_{T_{1}}^{2} \tilde{T}_{2}^{-2}, \tilde{T}_{1}\right)$. We apply $b$ on it to get a coherent (anti-coherent) polarized pair $\left(\tilde{X}^{2} \tilde{Y}^{-2}, \tilde{T}_{i}\right)$. Recall that $\left(\xi_{i}, \tilde{T}_{i}\right)$ is coherent with $\left(\xi_{1}, \tilde{T}_{1}\right)$. Thus by Proposition III.3.3 (or by Lemma III.4.1) $\tilde{X}^{2} \tilde{Y}^{-2}=\xi_{i}$ (or $\tilde{X}^{2} \tilde{Y}^{-2}=$ $\left.\xi_{i}^{-1} c\right)$, respectively. To get $\xi_{i}=\tilde{X}^{-2} \tilde{Y}^{2}$ from $\tilde{X}^{2} \tilde{Y}^{-2}=\xi_{i}^{-1} c$ we use (4) above.

The next Corollary is technical in nature, to be used later in order to obtain a smaller set of generators for $G$.

Corollary IV.4.2. (1) $\xi_{1}=\left(\tilde{T}_{2}\right)_{\tilde{T}_{1}^{-1}}^{2} \tilde{T}_{2}^{-2}$

(2) $\xi_{2}=\tilde{T}_{1}^{-2}\left(\tilde{T}_{1}\right)_{\tilde{T}_{2}^{-1}}^{2} \quad \xi_{2}=T_{4}^{2}\left(T_{4}\right)_{T_{2}^{-1}}^{-2} \quad \xi_{2}=\tilde{T}_{3}^{-2}\left(\tilde{T}_{3}\right)_{\tilde{T}_{2}^{-1}}^{2}$

(3) $\xi_{3}=\tilde{T}_{1}^{2}\left(\tilde{T}_{1}\right)_{\tilde{T}_{3}^{-1}}^{-2}$

(4) $\xi_{4}=\left(\tilde{T}_{4}\right)_{\tilde{T}_{6}^{-1}}^{2} \tilde{T}_{6}^{-2}$

(5) $\xi_{5}=\tilde{T}_{8}^{-2}\left(\tilde{T}_{8}^{2}\right)_{\tilde{T}_{7}^{-1}}$

(6) $\xi_{6}=\left(\tilde{T}_{4}\right)^{-2}\left(\tilde{T}_{4}^{2}\right)_{\tilde{T}_{6}^{-1}}, \quad \xi_{6}^{-1}=c \tilde{T}_{7}^{2}\left(\tilde{T}_{7}\right)_{T_{6}^{-1}}^{-2}$ 

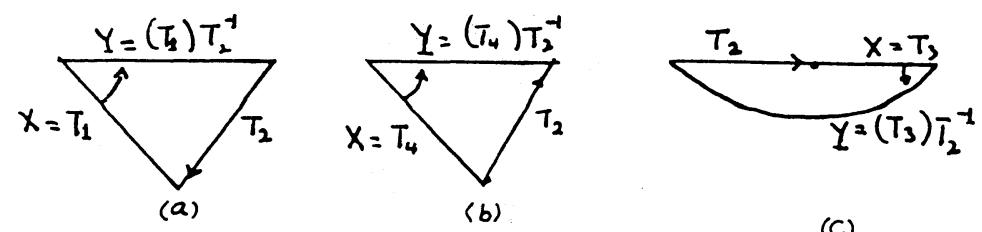

(C)

Figure IV.4.3.

(7) $\xi_{7}=\tilde{T}_{8}^{-2}\left(\tilde{T}_{8}\right)_{\tilde{T}_{7}^{-1}}^{2}$

(8) $\xi_{8}=\tilde{T}_{9}^{-2}\left(\tilde{T}_{9}\right)_{\tilde{T}_{8}^{-1}}^{2}$

(9) $\xi_{9}=\tilde{T}_{5}^{2}\left(\tilde{T}_{5}\right)_{\tilde{T}_{9}^{-1}}^{-2}$

(10) $\left(\xi_{4}\right)_{\tilde{T}_{2}^{-1}} \tilde{T}_{3} \tilde{T}_{7}^{-1} \tilde{T}_{8}=\xi_{5}$

(11) $\left(\xi_{2}\right)_{\tilde{T}_{4}} \tilde{T}_{3} \tilde{T}_{5}^{-1} \xi_{7}^{-1}=\xi_{8}$

(12) $\left(\xi_{3}\right)_{\tilde{T}_{2}^{-1}} \tilde{T}_{4} \tilde{T}_{5}^{-1} \tilde{T}_{8}=\xi_{7}$

Proof. In the entire proof we use Lemma IV.4.0(f) to interchange squares of consecutive half-twists, and multiplying the product by $c$. We also use the facts that $c^{2}=1, c \in \operatorname{Center}(G)$. The main tool is Lemma IV.4.1(6). We also use Lemma IV.4.0(d) to present an edge of a triangle as a conjugation of the other 2 edges.

(1) By definition.

(2) Consider the triangle from Fig. IV.4.3(a):

Since the polarization of $T_{2}$ goes from $Y$ to $X$, we apply Lemma IV.4.1(6) to get:

$$
\xi_{2}=X^{-2} Y^{2}=\tilde{T}_{1}^{-2}\left(\tilde{T}_{1}\right)_{\tilde{T}_{2}^{-1}}^{2}
$$

Consider the triangle from Fig. IV.4.3(b).

Since the polarization of $T_{2}$ goes from $X$ to $Y$, we apply Lemma IV.4.1(6) to get

$$
\xi_{2}=X^{2} Y^{-2}=\tilde{T}_{4}^{2}\left(\tilde{T}_{4}\right)_{\tilde{T}_{2}^{-1}}^{-2} .
$$

Consider the "triangle" from Fig. IV.4.3(c)

Since the polarization of $T_{2}$ goes from $Y$ to $X$, then:

$$
\xi_{2}=\tilde{T}_{3}^{-2}\left(\tilde{T}_{3}\right)_{T_{2}^{-1}}^{2}
$$




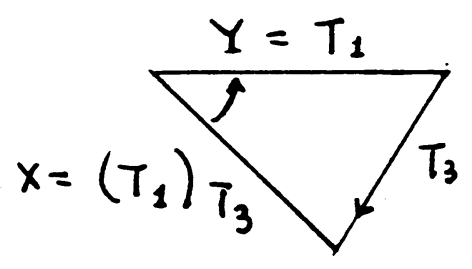

Figure IV.4.4.

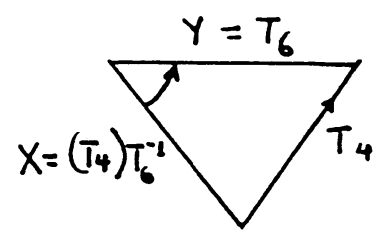

Figure IV.4.5.

(3) Consider the triangle from Fig. IV.4.4:

Since the polarization of $T_{3}$ goes from $Y$ to $X$, then:

$$
\xi_{3}=X^{-2} Y^{2}=\left(T_{1}\right)_{T_{3}}^{-2} T_{1}^{2} .
$$

We apply $\tilde{T}_{3}^{-2}$ on the above equation to get:

$$
\left(\xi_{3}\right)_{\tilde{T}_{3}^{-2}}=\left(\tilde{T}_{1}\right)_{\tilde{T}_{3}^{-1}}^{-2}\left(\tilde{T}_{1}\right)_{\tilde{T}_{3}^{-2}}^{2}
$$

By Lemma III.2.1, $\left(\xi_{3}\right)_{\tilde{T}_{3}^{-2}}=\xi_{3}$.

By Lemma IV.4.1(4), $\left(\tilde{T}_{1}^{2}\right)_{\tilde{T}_{3}^{-2}}=c T_{1}^{2}$.

Thus,

$$
\begin{aligned}
\xi_{3} & =c\left(T_{1}\right)_{T_{3}^{-1}}^{-2} T_{1}^{2} \\
& =T_{1}^{2}\left(T_{1}\right)_{T_{3}^{-1}}^{-2} \quad(\text { Lemma IV.4.0(5)) }
\end{aligned}
$$

(4) Consider the triangle from Fig. IV.4.5:

Since the polarization of $T_{4}$ goes from $X$ to $Y$

$$
\xi_{4}=\left(\tilde{T}_{4}\right)_{\tilde{T}_{6}^{-1}}^{2} \tilde{T}_{6}^{-2} .
$$

(5) Consider the triangle from Fig. IV.4.6: Since the polarization of $T_{5}$ goes from $X$ to $Y$

$$
\xi_{5}=\left(\tilde{T}_{8}\right)_{\tilde{T}_{5}}^{2} \tilde{T}_{8}^{-2}
$$




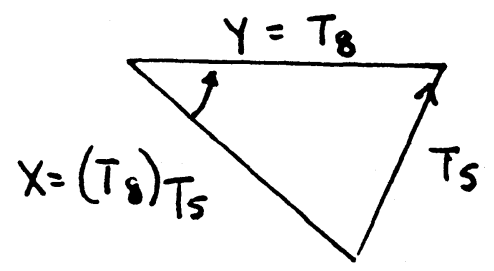

Figure IV.4.6.
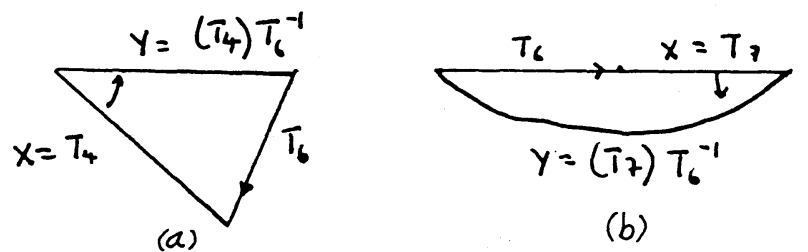

(b)

Figure IV.4.7.

(6) Consider the triangle from Fig. IV.4.7(a):

Since the polarization of $T_{6}$ goes from $Y$ to $X$

$$
\xi_{6}=\tilde{T}_{4}^{-2}\left(\tilde{T}_{4}^{2}\right)_{T_{6}^{-1}}
$$

Consider the "triangle" from Fig. IV.4.7(b).

Since the polarization of $T_{6}$ goes from $Y$ to $X$

$$
\xi_{6}=\tilde{T}_{7}^{-2}\left(\tilde{T}_{7}^{2}\right)_{T_{6}^{-1}} \Rightarrow \xi_{6}^{-1}=c \tilde{T}_{7}^{2}\left(\tilde{T}_{7}^{-2}\right)_{\tilde{T}_{6}^{-1}}
$$

(7) Consider the "triangle" from Fig. IV.4.8:

Since the polarization of $T_{7}$ goes from $Y$ to $X$

$$
\xi_{7}=\tilde{T}_{8}^{-2}\left(\tilde{T}_{8}^{2}\right)_{T_{7}^{-1}}
$$

(8) Similar to (7).

(9) Similar to (3).

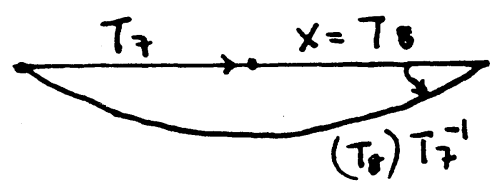

Figure IV.4.8. 
For (10)-(12) we shall only prove the first assertion. The others are similar. By Lemma III.2.2, $\left(\left(\xi_{2}\right)_{\tilde{T}_{4}} \tilde{T}_{3} \tilde{T}_{5}^{-1} \tilde{T}_{7}^{-1},\left(T_{2}\right)_{\tilde{T}_{4}} \tilde{T}_{3} \tilde{T}_{5}^{-1} \tilde{T}_{7}^{-1}\right)$ is a polarized pair coherent with $\left(\xi_{2}, \tilde{T}_{2}\right)$. By Lemma IV.2.3 $\left(\tilde{T}_{2}\right)_{\tilde{T}_{4}} \tilde{T}_{3} \tilde{T}_{5}^{-1} \tilde{T}_{7}^{-1}=\tilde{T}_{8}$, preserving polarization. Thus $\left(\left(\xi_{2}\right)_{\tilde{T}_{4}} \tilde{T}_{3} \tilde{T}_{5}^{-1} \tilde{T}_{7}^{-1}, \tilde{T}_{8}\right)$ is coherent with $\left(\xi_{2}, \tilde{T}_{2}\right)$. But also $\left(\xi_{8}, \tilde{T}_{8}\right)$ is coherent with $\left(\xi_{2}, \tilde{T}_{2}\right)$. From uniqueness, $\left(\xi_{2}\right)_{\tilde{T}_{4}} \tilde{T}_{3} \tilde{T}_{5}^{-1} \tilde{T}_{7}^{-1}=\xi_{8}$.

\section{IV.5. $\eta$-situation.}

In $\S 3$ we constructed a homomorphism of groups $\alpha: \tilde{B}_{9} \rightarrow G$ s.t. $\alpha\left(\tilde{T}_{i}\right)=$ $E_{i}$.

We introduce $G$ as a $\tilde{B}_{9}$-group by $(g) \tilde{Y}=g_{\alpha(\tilde{Y})}\left(=\alpha(\tilde{Y})^{-1} g \alpha(\tilde{Y})\right)$.

The homomorphism $\alpha$ here then becomes a homomorphism of $\tilde{B}_{9}$-groups.

Claim IV.5.1. Let $\mu=\alpha(c), \quad c=\left[T_{2}^{2}, T_{1}^{2}\right]$, then:

(a) $\mu=\left[E_{k}^{2}, E_{\ell}^{2}\right] \forall k, \ell$ s.t. $T_{k}$ and $T_{\ell}$ are adjacent.

(b) Let $\rho$ be any automorphism of the type $\rho_{m_{1}, m_{2}, m_{3}, m_{4}, m_{6}, m_{9}}$ s.t. $\exists j_{0}$ with $m_{j_{0}} \neq 0$ and all other $m_{i}=0$. Then $(\mu) \rho=\mu$.

(c) $\mu \in \operatorname{Center}(G)$.

(d) $\mu=\left[\left(E_{k}^{2}\right) \rho_{k}^{m} .\left(E_{\ell}\right)^{2} \rho_{\ell}^{n}\right] \quad \forall k, \ell$ s.t. $T_{k}$ and $T_{\ell}$ are adjacent, and $(k, \ell) \neq$ $(3,7),(2,8),(4,5)$.

(e) $\mu^{2}=1$.

(f) If $\tilde{X}$ and $\tilde{Y}$ are 2 consecutive half-twists, then $\alpha(\tilde{X})^{2} \alpha(\tilde{Y})^{2}=$ $\mu \alpha(\tilde{Y})^{2} \alpha(\tilde{X})^{2}$.

Proof.

(a) $c=\left[T_{k}^{2}, T_{\ell}^{2}\right] \forall k, \ell$ s.t. $\tilde{T}_{k}$ and $\tilde{T}_{\ell}$ are adjacent (Claim IV.4.1 (4)). Then $\mu=\alpha(c)=\alpha\left[\tilde{T}_{k}^{2}, \tilde{T}_{\ell}^{2}\right]=\left[E_{k}^{2}, E_{\ell}^{2}\right] \quad \forall k, \ell$ such that $\tilde{T}_{k}$ and $\tilde{T}_{\ell}$ are adjacent.

(b) $\rho=\rho_{1}^{m_{1}}\left(\rho_{2} \rho_{8}\right)^{m_{2}}\left(\rho_{3} \rho_{2}\right)^{m_{3}}\left(\rho_{4} \rho_{5}\right)^{m_{4}} \rho_{6}^{m_{6}} \rho_{9}^{m_{9}}$, and $\left(E_{i}\right) \rho=E_{i}$ if $\rho_{i}$ appears in $\rho$ to the power 0 . If $j_{0} \neq 1,2$, then $\left(E_{1}\right) \rho=E_{1}$ and $\left(E_{2}\right) \rho=E_{2}$. We take $\mu=\left[E_{1}^{2}, E_{2}^{2}\right]$ and apply $\rho$ on it to get $(\mu) \rho=\left[E_{1}^{2}, E_{2}^{2}\right] \rho=\left[\left(E_{1}^{2}\right) \rho,\left(E_{2}^{2}\right) \rho\right]=\left[E_{1}^{2}, E_{2}^{2}\right]=\mu$. If $j_{0}=1$ or 2 , we apply $\rho$ on $\mu=\left[E_{3}^{2}, E_{5}^{2}\right]$ and continue similarly to get the result. 
(c) Since $c \in \operatorname{Center}\left(\tilde{B}_{9}\right)$, then $\mu \in \operatorname{Center}\left(\alpha\left(\tilde{B}_{9}\right)\right)$. Since $\alpha\left(\tilde{T}_{i}\right)=E_{i}$, we get $\left[\mu, E_{i}\right]=1 \quad \forall i$. We want to prove $\left[\mu, E_{i^{\prime}}\right]=1 \quad \forall i$ and then we get $\mu \in \operatorname{Center}(G)$. ( $G$ is generated by $\left\{E_{j}, E_{j^{\prime}}\right\}_{j=1}^{9}$.) Take $\rho=$ $\rho_{1}^{m_{1}}\left(\rho_{2} \rho_{8}\right)^{m_{2}}\left(\rho_{3} \rho_{7}\right)^{m_{3}}\left(\rho_{4} \rho_{5}\right)^{m_{4}} \rho_{6}^{m_{6}} \rho_{9}^{m_{9}}$ such that $\rho_{i}$ appears in $\rho$ to the power 1 and all other $m_{i}=0$, Thus, $\left(E_{i}\right) \rho=E_{i^{\prime}}$ and there is exactly one $m_{j_{0}} \neq 0$. Thus, by $(b)(\mu) \rho=\mu$. Thus,

$$
1=(1) \rho=\left[E_{i}, \mu\right] \rho=\left[\left(E_{i}\right) \rho,(\mu) \rho\right]=\left[E_{i^{\prime}}, \mu\right] .
$$

(d) Since $(k, \ell) \neq(2,8),(3,7),(4,5)$, then $L_{k}$ and $L_{\ell}$ are not on the same line. Let $\rho^{\prime}=\rho_{1}^{m_{1}}\left(\rho_{2} \rho_{8}\right)^{m_{2}}\left(\rho_{3} \rho_{7}\right)^{m_{2}}\left(\rho_{4} \rho_{5}\right)^{m_{5}} \rho_{6}^{m_{6}} \rho_{9}^{m_{6}}$ where $\rho_{k}$ appears to the power $m$ and all other $m_{i}=0$. In particular, $\rho_{\ell}$ appears to the power $0 \cdot((k, \ell) \neq(2,8),(3,7),(4,5))$. Then $\left(E_{k}\right) \rho=\left(E_{k}\right) \rho_{k}^{m},\left(E_{\ell}\right) \rho=$ $E_{\ell}$. Let $\rho^{\prime}$ be as above s.t. $\rho_{\ell}$ appears there to the power $n$ and all other $m_{i}=0$. Then, $\left(E_{\ell}\right) \rho^{\prime}=\left(E_{\ell}\right) \rho_{\ell}^{n},\left(E_{k}\right) \rho^{\prime}=E_{k}$. Thus, $\mu=(\mu) \rho \rho^{\prime}=$ $\left[\left(E_{k}\right) \rho_{k}^{m},\left(E_{\ell}\right) \rho_{\ell}^{n}\right]$.

(e) $\mu^{2}=\alpha(c)^{2}=\alpha\left(c^{2}\right)=\alpha(1)=1$.

(f) From Lemma IV.4.1 (4).

Corollary IV.5.2. $\eta_{1}=\alpha\left(\xi_{1}\right)$ is a prime element in $G$ with s.h.t. $\tilde{T}_{1}$ and central element $\mu$.

Proof. $\xi_{1}$ is a prime element of $\tilde{B}_{9}$ with s.h.t. $\tilde{T}_{1}$ (see Claim IV.4.1). Thus, $\eta_{1}=\alpha\left(\xi_{1}\right)$ is a prime element of $\alpha\left(\tilde{B}_{9}\right)$ with s.h.t. $\tilde{T}_{1}$ and central element $\mu=\alpha(c)$, from the previous lemma, $\mu \in \operatorname{Center}(G)$. Thus, $\eta_{i}$ is a prime element of $G$.

\section{$\eta$-situation.}

Consider $G$ as a $\tilde{B}_{9}$-group.

Let $\eta_{1}=\alpha\left(\xi_{1}\right)$ be a prime element of $G$ with s.h.t. $\tilde{T}_{1}$ and central element $\mu=\alpha(c)$.

Let $\eta_{i}=L_{\eta_{1}, \tilde{T}_{1}}\left(\tilde{T}_{i}\right)$ be the unique prime element of $G$ such that $\left(\eta_{i}, \tilde{T}_{i}\right)$ is coherent with $\left(\eta_{1}, \tilde{T}_{1}\right)$.

Claim IV.5.3. Consider the $\eta$-situation. Then: 
(a) $\left(\eta, \tilde{T}_{i}\right)$ is a polarized pair of $G$ with s.h.t. $\tilde{T}_{i}$ and corresponding element $\mu$. In particular, $\eta_{i}$ is a prime element with s.h.t. $\tilde{T}_{i}$.

(b) $\eta_{i}=\alpha\left(\xi_{i}\right)$.

(c) Every $\eta_{i}$ is of the form $\alpha\left(X^{2} Y^{-2}\right)$ where $X, Y$ are adjacent half-twists and the s.h.t. $T_{i}$ is $X Y X^{-1}\left(=Y^{-1} X Y\right)$.

(d) $\eta_{1}=\left(E_{2}\right)_{E_{1}^{-1}}^{2} E_{2}^{-2}$.

$\eta_{2}=E_{1}^{-2}\left(E_{1}\right)_{E_{2}^{-1}}^{2}$

$\eta_{3}=E_{1}^{2}\left(E_{1}\right)_{E_{3}^{-1}}^{-2}$.

$\eta_{4}=\left(E_{4}\right)_{E_{6}^{-1}}^{2} E_{6}^{-2}$.

$\eta_{5}=\left(E_{8}\right)_{E_{5}}^{2} E_{8}^{-2}$.

$\eta_{6}=\left(E_{4}\right)^{-2}\left(E_{4}\right)_{E_{6}^{-1}}^{2} \quad \eta_{6}^{-1}=\mu E_{7}^{2}\left(E_{7}\right)_{E_{6}^{-1}}^{-2}$.

$\eta_{7}=E_{8}^{-2}\left(E_{8}\right)_{E_{7}^{-1}}^{2}$

$\eta_{8}=E_{9}^{-2}\left(E_{9}\right)_{E_{8}^{-1}}^{2}$

$\eta_{9}=E_{5}^{2}\left(E_{5}\right)_{E_{9}^{-1}}^{-2}$.

$\eta_{5}=\left(\eta_{4}\right)_{\tilde{T}_{2}^{-1}} \tilde{T}_{3} \tilde{T}_{7}^{-1} \tilde{T}_{8}$

$\eta_{7}=\left(\eta_{3}\right)_{\tilde{T}_{2}^{-1}} \tilde{T}_{4} \tilde{T}_{5}^{-1} \tilde{T}_{8}$

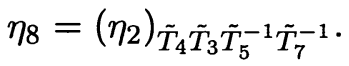

Proof.

(a) By the construction of $\eta_{i}$, the pairs $\left(\eta_{i}, \tilde{T}_{i}\right)$ are coherent polarized pairs. $\left(\eta_{1}, \tilde{T}_{1}\right)$ has $\mu$ as central element and coherent pairs have the same central element (Corollary III.3.1).

(b) $\xi_{1}$ is a prime element with s.h.t. $\tilde{T}_{i}$ s.t. $\left(\xi_{i}, \tilde{T}_{i}\right)$ coherent with $\left(\xi_{1}, \tilde{T}_{1}\right)$. Thus, $\alpha\left(\xi_{i}\right)$ is a prime element with s.h.t. $\tilde{T}_{i}$ s.t. $\left(\alpha\left(\xi_{i}\right), \tilde{T}_{i}\right)$ is coherent with $\left(\alpha\left(\xi_{1}\right), \tilde{T}_{1}\right)=\left(\eta_{1}, \tilde{T}_{1}\right)$. From uniqueness $\alpha\left(\xi_{i}\right)=\eta_{i}$. 
(c) From Lemma IV.4.1.(6).

(d) Apply $\alpha$ on the formulas of Corollary IV.4.2.

\section{IV.6. N-situation.}

\section{N-situation.}

Let $\mathcal{G}$ be a $\tilde{B}_{9}$-group.

Assume there exists a $\tilde{B}_{9}$-homomorphism $\lambda: \tilde{B}_{9} \rightarrow \mathcal{G}$ s.t. $(g) b=g_{\lambda(b)} \quad \forall b \in \tilde{B}_{9}, \forall g \in \mathcal{G}$.

Let $f$ be a prime element in $\mathcal{G}$ with s.h.t. $\tilde{T}_{1}$ and central element $\nu$.

Let $\left.f_{i}=L_{\left\{\left(f, \tilde{T}_{1}\right)\right\}}\left(\tilde{T}_{i}\right)\right\}$ be the unique prime element s.t. $\left(f_{i}, \tilde{T}_{1}\right)$ is coherent with $\left(f_{i}, \tilde{T}_{1}\right) i=1, \ldots, 9$.

By Corollary III.3.1, $\nu$ is the central element of $f_{i} \forall i$.

Let $\eta_{1}=\lambda\left(\xi_{1}\right), \eta_{1}$ is a prime element with s.h.t. $\tilde{T}_{1}$ and central element $\mu=\lambda(c)$.

Let $\eta_{i}=L_{\left\{\eta_{1}, \tilde{T}_{1}\right\}}\left(\tilde{T}_{i}\right)$.

It is easy to see that, similar to the situation in Claim IV.5.3, $\eta_{i}=\lambda\left(\xi_{i}\right)$.

Let $\mu=\lambda(c)$ be the central element of $\eta_{i}, \forall i$.

Let $N_{1}=\left\{\eta_{i} \mid\left(\eta_{i}, \tilde{T}_{i}\right)\right.$ be a polarized pair coherent with $\left(\eta_{1}, \tilde{T}_{1}\right), i=$ $1 \ldots 9\}$.

Let $N_{2}=\left\{f_{i} \mid\left(f_{i}, \tilde{T}_{i}\right)\right.$ be a polarized pair coherent with $\left(f, \tilde{T}_{1}\right), \quad i=$ $1 \ldots 9\}$.

\section{Definitions.}

Let $a, b \in N_{1} \cup N_{2}$. We say that $a$ and $b$ are weakly disjoint (transversal, disjoint, adjacent, consecutive or cyclic) if their s.h.t. are weakly disjoint (transversal, disjoint, adjacent, consecutive or cyclic respectively). (See definitions in the beginning of Chapter III.)

Let $a \in N_{1} \cup N_{2}$ with s.h.t. $\tilde{X}$. Let $\tilde{Z} \in \tilde{B}_{9}$. We say that $a$ and $\tilde{Z}$ are weakly disjoint (transversal, disjoint, adjacent, consecutive, cyclic) if $X$ and $Z$ are weakly disjoint (transversal, disjoint, adjacent, consecutive, cyclic respectively).

Lemma IV.6.1. $a \in N_{1} \cup N_{2}, \tilde{Z} \in \tilde{B}_{9} \mu=\lambda(c)$, then: 
(i) $a, \tilde{Z}$ are adjacent $\Rightarrow a_{\tilde{Z}^{2}}= \begin{cases}a \mu & a \in N_{1} \Rightarrow \\ a \nu & a \in N_{2}\end{cases}$

(ii) $a, \tilde{Z}$ are weakly disjoint or commonly supported $\Rightarrow a_{\tilde{Z}^{2}}=a$.

In other words,

$$
\left[\lambda\left(\tilde{Z}^{2}\right), a^{ \pm 1}\right]=\left[\lambda\left(\tilde{Z}^{-2}\right), a^{ \pm 1}\right]= \begin{cases}\mu & a \in N_{1}, a, Z \text { adjacent } \\ \nu & a \in N_{2}, a, Z \text { adjacent } \\ 1 & a, Z \text { weakly disjoint or cyclic. }\end{cases}
$$

Proof. Let $\tilde{X}$ be the s.h.t. of $a$.

(i) There exists $v \in P_{9}$ s.t. $Y=v^{-1} Z v$ is consecutive to $X$. By the definition of prime element:

$$
a_{\tilde{Y}^{-2}}= \begin{cases}a \mu & a \in N_{1} \\ a \nu & a \in N_{2} .\end{cases}
$$

On the other hand, $Y^{-2}=v^{-1} Z^{-2} v Z^{2} Z^{-2}=\left[v^{-1}, Z^{-2}\right] Z^{-2}$. Since $v \in P_{9}$ and $Z^{2} \in P_{9}$, then $\left[\tilde{v}^{-1}, \tilde{Z}^{-2}\right] \in \tilde{P}_{9}$. But $\tilde{P}_{9}^{\prime}$ is generated by $c$ (Lemma III.5.2), $c^{2}=1$, so $\tilde{Y}^{-2}=c^{\varepsilon} \tilde{Z}^{-2} \varepsilon=0,1$. Thus, $a_{\tilde{Y}^{-2}}=a_{\lambda\left(\tilde{Y}^{-2}\right)}=$ $a_{\lambda\left(c^{\varepsilon}\right) \lambda\left(\tilde{Z}^{-2}\right)}=a_{\mu^{\varepsilon} \lambda\left(\tilde{Z}^{-2}\right)}=a_{\lambda\left(\tilde{Z}^{-2}\right)}=a_{\tilde{Z}^{-2}}$. So, $a_{\tilde{Z}^{-2}}=a_{\tilde{Y}^{-2}}$. Thus,

$$
a_{\tilde{Z}^{-2}}= \begin{cases}a \mu & a \in N_{1} \\ a \nu & a \in N_{2}\end{cases}
$$

Since $\nu, \mu \in \operatorname{Center}(G)$

$$
a_{\tilde{Z}^{2}}= \begin{cases}a \mu & a \in N_{1} \\ a \nu & a \in N_{2} .\end{cases}
$$

(ii) If $a$ and $Z$ are weakly disjoint (cyclic), then: There exists $v \in P_{9}$ s.t. $Y=v^{-1} Z v$ and $X$ are disjoint (commonly supported). As above, $a_{\tilde{Y}^{2}}=a_{\tilde{Z}^{2}}$. By the definition of prime element $a_{\tilde{Y}^{2}}=1$. Thus, $a_{\tilde{Z}^{2}}=1$.

Proposition IV.6.2. (i) If $a, b \in N_{1} \cup N_{2}$ are adjacent, then

$$
\left[a, b^{ \pm 1}\right]= \begin{cases}\mu & a, b \in N_{1} \\ \nu & \text { otherwise. }\end{cases}
$$

(ii) If $a, b \in N_{1} \cup N_{2}$ are commonly supported or weakly disjoint, then

$$
\left[a, b^{ \pm 1}\right]=1
$$



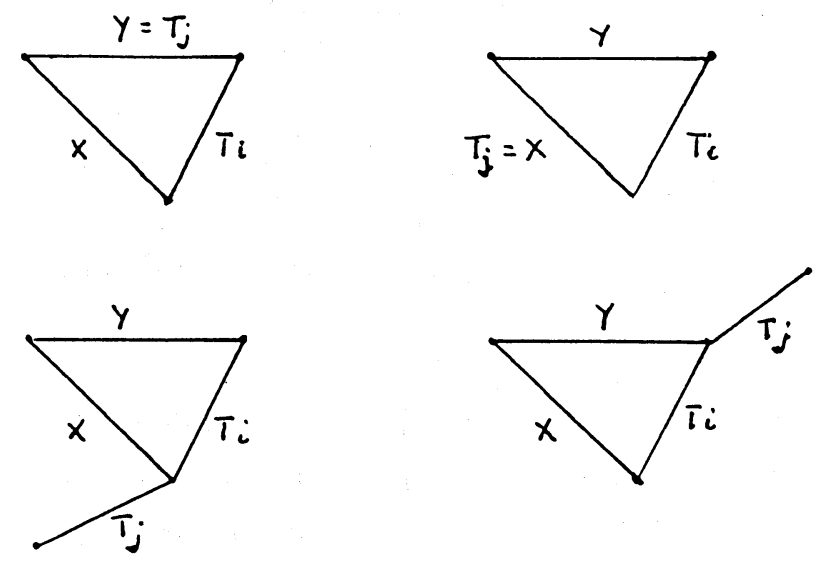

Figure IV.6.

Proof. For $a, b \in N_{1}$ and $a, b \in N_{2}$, the proof follows from Lemma III.4.2. Consider the case $a \in N_{1}, b \in N_{2}$. Assume $a=\eta_{i}$ with s.h.t. $\tilde{T}_{i}$, and $b \in N_{2}$ with s.h.t. $\tilde{T}_{j}$. By Claim IV.4.1(6), $a$ is of the form $\lambda\left(\tilde{X}^{2} \tilde{Y}^{-2}\right)$ where $X$ and $Y$ are consecutive half-twists s.t. $X, Y, T_{i}$ create a triangle as in Fig. IV.4.2.

$$
\text { Now, }\left[a, b^{ \pm 1}\right]=\left[\lambda\left(\tilde{X}^{2} \tilde{Y}^{-1}\right), b^{ \pm 1}\right]=\left[\lambda\left(\tilde{Y}^{-2}\right), b^{-1}\right]_{\lambda\left(\tilde{X}^{-2}\right)} \cdot\left[\lambda\left(\tilde{X}^{2}\right), b^{ \pm 1}\right] .
$$

( by Claim II.4). By the previous Lemmas and the fact that $\nu \in \operatorname{Center}(G)$, we get:

$$
\begin{gathered}
{\left[\lambda\left(\tilde{Y}_{2}^{-2}\right), b^{ \pm 1}\right]_{\lambda\left(\tilde{X}_{2}^{2}\right)}=\left\{\begin{array}{cc}
\nu & Y \text { and } T_{j} \text { are adjacent } \\
1 & Y \text { and } T_{j} \text { are weakly disjoint or } \\
\text { commonly supported. }
\end{array}\right.} \\
{\left[\lambda\left(X_{2}^{-2}\right), b^{ \pm 1}\right]=\left\{\begin{array}{cc}
\nu & X \text { and } T_{j} \text { are adjacent } \\
1 & X \text { and } T_{j} \text { are weakly disjoint or } \\
& \text { commonly supported. }
\end{array}\right.}
\end{gathered}
$$

These are the only possible values since $Y$ (or $X$ ) are never cyclic with $T_{j}$. Since $\nu^{2}=1$, in order to get $\left[a, b^{ \pm 1}\right] \neq 1$, we need one factor to be $\nu$ and the other one to be 1 . Thus we need $T_{j}$ adjacent to $Y$, and $T_{j}$ is weakly disjoint or commonly supported to $X$, (or vice-versa). This can happen in four different cases (Fig. IV.6). But in all of the four cases $T_{j}$ is adjacent to $T_{i}$ (see Fig. IV.6). 
Lemma IV.6.3. Consider the $N$-situation. Then:

$$
\begin{aligned}
&\left(f_{i}\right)_{\tilde{T}_{k}}=\left\{\begin{array}{lll}
f_{i}^{-1} \nu & k=i \\
f_{i} & T_{i}, T_{k} & \text { weakly disjoint } \\
f_{k} f_{i} & T_{i}, T_{k} & \text { orderly adjacent } \\
f_{i} f_{k}^{-1} & T_{i}, T_{k} & \text { are not orderly adjacent. }
\end{array}\right. \\
&\left(f_{i}\right)_{\tilde{T}_{k}^{-1}}=\left\{\begin{array}{lll}
f_{i}^{-1} \nu & k=i \\
f_{i} & T_{i}, T_{k} & \text { weakly disjoint } \\
f_{i} f_{k} & T_{i}, T_{k} & \text { orderly adjacent } \\
f_{k}^{-1} f_{i} & T_{i}, T_{k} & \text { are not orderly adjacent. }
\end{array}\right.
\end{aligned}
$$

Proof. Recall that $f_{i}$ is a prime element with s.h.t. $T_{i}$. Moreover, $\nu \in$ Center $(G), \nu=\left[f_{i}^{ \pm 1}, f_{j}^{ \pm 1}\right]$ for $T_{i}, T_{j}$ adjacent half-twists (previous Lemma).

One can see from Fig. IV.1.6 that if $T_{i}$ and $T_{k}$ are weakly disjoint then they are disjoint unless $k=4$ and $i=3,7$, in which case they are transversal. We shall treat separately the case where $T_{i}$ and $T_{k}$ are disjoint and the case where $T_{i}$ and $T_{k}$ are transversal.

For $k=i$ and for $T_{k}$ and $T_{i}$ disjoint we get from the definition of prime element that:

$$
\begin{array}{ll}
\left(f_{i}\right)_{\tilde{T}_{k}^{-1}}=f_{k}^{-1} \mu & i=k \\
\left(f_{i}\right)_{\tilde{T}_{k}^{-1}}=f_{i} & \text { for } T_{k} \text { and } T_{i} \text { disjoint. }
\end{array}
$$

We conjugate the above formulas by $\tilde{T}_{k}$ to get the correct formula for $\left(f_{i}\right)_{\tilde{T}_{k}}$ where $k=i$ or when $T_{i}$ and $T_{k}$ are weakly disjoint.

For $T_{i}$ and $T_{k}$ orderly adjacent we use Proposition III.4.1 to get: $\left(f_{i}\right)_{\tilde{T}_{k}^{-1}}=f_{i} f_{k}$.

Thus, $\left(f_{i}\right)_{\tilde{T}_{k}^{-2}}=\left(f_{i}\right)_{\tilde{T}_{k}^{-1}}\left(f_{k}\right)_{\tilde{T}_{k}^{-1}}=f_{i} f_{k} f_{k}^{-1} \nu=f_{i} \nu$.

Since $\nu^{2}=1, \nu \in \operatorname{Center}(G)$, we get: $f_{i}=\nu^{-1}\left(f_{i}\right)_{\tilde{T}_{k}^{-2}}=\left(f_{i}\right)_{\tilde{T}_{k}^{-2}} \nu$.

Thus, $\left(f_{i}\right)_{T_{k}}=\left(f_{i}\right)_{T_{k}^{-1}} \nu=f_{i} f_{k} \nu=f_{i} f_{k}\left[f_{k}^{-1}, f_{i}^{-1}\right]=f_{i} f_{k} f_{k}^{-1} f_{i}^{-1} f_{k} f_{i}=$ $f_{k} f_{i}$.

For $T_{i}$ and $T_{k}$ be non-orderly adjacent, we use III.4.1 again to get: $\left(f_{i}\right)_{\tilde{T}_{k}^{-1}}=f_{k}^{-1} f_{i}$.

Thus, $\left(f_{k}\right)_{\tilde{T}_{k}^{-2}}=\left(f_{k}\right)_{\tilde{T}_{k}^{-1}}^{-1}\left(f_{i}\right)_{\tilde{T}_{k}^{-1}}=\nu^{-1} f_{k} f_{k}^{-1} f_{i}=\nu f_{i} \Rightarrow$

$\left(f_{i}\right)_{T_{k}}=\nu\left(f_{i}\right)_{T_{k}^{-1}}=\nu f_{k}^{-1} f_{i}=\left[f_{i}, f_{k}^{-1}\right] f_{k}^{-1} f_{i}=f_{i} f_{k}^{-1} f_{i}^{-1} f_{k} f_{k}^{-1} f_{i}=f_{i} f_{k}^{-1}$. 
For $T_{i}$ and $T_{k}$ transversal we get from Lemma III.4.2 that

$$
\left(f_{i}\right)_{\tilde{T}_{k}^{-1}}=f_{i} /
$$

We conjugate the above formula by $\tilde{T}_{k}$ to get the correct formula for $\left(f_{i}\right)_{\tilde{T}_{k}}$ for $T_{i}$ and $T_{k}$ transversal.

IV.7. New set of generators for $\mathbf{G}:\left\{\mathbf{A}_{\mathbf{j}}, \mathbf{E}_{\mathbf{j}}\right\}$.

Recall

$$
\begin{aligned}
& E_{i}= \begin{cases}\Gamma_{i} & i \neq 2,7 \\
\Gamma_{i} & i=2,7\end{cases} \\
& E_{i}^{\prime}=\left(E_{i}\right) \rho_{i} \\
& A_{j}=E_{j}^{\prime} E_{j}^{-1} .
\end{aligned}
$$

Claim IV.7.1. $\left\{A_{j}, E_{j}\right\}_{j=1}^{9}$ generates $G$.

Proof. By Lemma II.2, $\left\{E_{j}, E_{j^{\prime}}\right\}_{j=1}^{9}$ generates $G$. Since $E_{j^{\prime}}=A_{j} E_{j}$, $\left\{A_{j}, E_{j}\right\}_{j=1}^{9}$ generates $G$.

Definition. $H_{1}=\tilde{B}_{n}$-orbit of $A_{1}$.

Proposition IV.7.2. $A=A_{1}$ is a prime element of $H_{1}$ with s.h.t. $\tilde{T}_{1}$.

Proof. Consider the following frame of $B_{9}: X_{1}=T_{1}, X_{2}=T_{3}, X_{3}=$ $\left(T_{5}\right)_{9}, X_{4}=T_{9} . X_{5}=T_{8}, X_{6}=T_{7}, X_{7}=T_{6}, X_{8}=\left(T_{2}\right)_{T_{3}^{-1} T_{5}^{-1} T_{8}^{-1} T_{7}^{-1} T_{6}^{-1}}$ (see Fig. IV.7.1).

In order to prove that $H_{1}$ is a prime element of $A_{1}$, we shall prove that all the necessary conditions of Proposition III.7.1 are fulfilled using the above frame of $B_{9}$. Let $\nu=A \cdot A_{\tilde{T}_{1}^{-1}}$. It is easy to see that $\nu=E_{1^{\prime}}^{2} E_{1}^{-2}$. $\left(\nu=A \cdot A_{\tilde{T}_{1}^{-1}}=A \cdot A_{E_{1}^{-1}}=E_{1^{\prime}} E_{1}^{-1} E_{1} E_{1^{\prime}} E_{1}^{-1} E_{1}^{-1}=E_{1^{\prime}}^{2} E_{1}^{-2}\right.$.

(0) By definition of $H_{1}, H_{1}$ is the full orbit of $A_{1}=A$. 


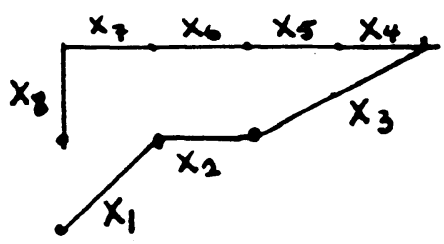

Figure IV.7.1.

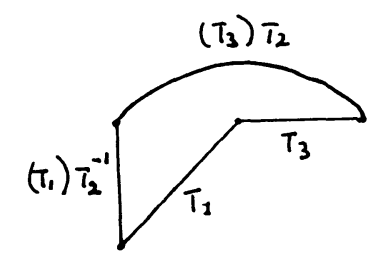

Figure IV.7.2.

(1) We have to prove $A_{\tilde{X}_{2}^{-1} \tilde{X}_{1}^{-1}}=A^{-1} A_{\tilde{X}_{2}^{-1}}$.

(1a) Since $A E_{1}=E_{1}^{\prime}$, we can use Proposition II.6 and Corollary II.3 to get $\left\langle A E_{1}, E_{3}\right\rangle=\left\langle E_{1}, E_{3}\right\rangle=1$. Thus, by Lemma II.4(h) we get $A_{E_{2}^{-1} E_{1}^{-1}}=$ $A^{-1} \cdot A_{E_{3}^{-1}}$. Since $A_{\tilde{X}}=A_{\alpha(\tilde{X})}$ and $\alpha\left(\tilde{X}_{1}\right)=\alpha\left(\tilde{T}_{1}\right)=E_{1}, \alpha\left(\tilde{X}_{2}\right)=$ $\alpha\left(\tilde{T}_{3}\right)=E_{3}$, we get (1a) .

(1b) $A_{\tilde{X}_{1}}=A_{\tilde{T}_{1}}=A_{\alpha\left(\tilde{T}_{1}\right)}=A_{E_{1}}$. Thus, $A_{\tilde{X}_{1}} E_{1}=A_{E_{1}} E_{1}=E_{1}^{-1} A E_{1} E_{1}=$ $E_{1}^{-1} E_{1}^{\prime} E_{1}^{-1} E_{1} E_{1}=E_{1}^{-1} E_{1}^{\prime} E_{1}=\left(E_{1}\right) \rho_{1}^{-1}$. By Proposition II.6 and Corollary II.3 we get $\left\langle A_{\tilde{X}_{1}} E_{1}, E_{3}\right\rangle=\left\langle E_{1}, E_{3}\right\rangle=1$. By Lemma II.4.(h) we get $A_{\tilde{X}_{1} E_{3}^{-1} E_{1}^{-1}}=A_{\tilde{X}_{1}}^{-1} A_{\tilde{X}_{1} E_{3}^{-1}}$. Since $\alpha\left(\tilde{X}_{1}\right)=E_{1}$ and $\alpha\left(\tilde{X}_{2}\right)=\alpha\left(\tilde{T}_{3}\right)=E_{3}$, we get $(1 \mathrm{~b})$.

(2a) We need to prove $\nu_{\tilde{X}_{1}^{2}}=\nu$. Since $\alpha\left(\tilde{X}_{1}\right)=\alpha\left(\tilde{T}_{1}\right)=E_{1}$, then $\nu_{\tilde{X}_{1}^{2}}=\nu_{E_{1}^{2}}$. To prove $\nu_{E_{1}^{2}}=\nu$, it is enough to prove $\left[E_{1^{\prime}}^{2}, E_{1}^{2}\right]=1$.

Consider the following 4 half-twists in $B_{9}: T_{1}, T_{3}, T_{2}^{-1} T_{3} T_{2}, T_{2} T_{1} T_{2}^{-1}$. Obviously, the above half-twist consists of a "good triangle" in $\tilde{B}_{9}$ (see Fig. IV.7.2).

By Lemma III.1.2,

$$
\text { and } \begin{aligned}
\tilde{T}_{1} \cdot \tilde{T}_{2}^{-1} \tilde{T}_{3} \tilde{T}_{2} & =\tilde{T}_{2}^{-1} \tilde{T}_{3} \tilde{T}_{2} \cdot \tilde{T}_{1} \\
\tilde{T}_{1}^{2} \cdot \tilde{T}_{2}^{-1} \tilde{T}_{3}^{2} \tilde{T}_{2} & =\tilde{T}_{3}^{2} \cdot \tilde{T}_{2} \tilde{T}_{1}^{2} \tilde{T}_{2}^{-1}
\end{aligned}
$$


By Lemma IV.3.4, there exist $\alpha: \tilde{B}_{9} \rightarrow G$ s.t. $\alpha\left(T_{i}\right)=E_{i}$. Thus,

$$
\text { and } \quad \begin{aligned}
& {\left[E_{1}, E_{2}^{-1} E_{3} E_{2}\right]=1} \\
& E_{1}^{2} \cdot E_{2}^{-1} E_{3}^{2} E_{2}=E_{3}^{2} E_{2} \cdot E_{1}^{2} E_{2}^{-1} .
\end{aligned}
$$

Thus, $E_{1}^{2}=E_{3}^{2} \cdot E_{2} E_{1}^{2} E_{2}^{-1} \cdot E_{2}^{-1} E_{3}^{-2} E_{2}$.

We shall find the commutator of $E_{1}^{2}$, with each of the 3 factors $\alpha=E_{3}$, $\beta=E_{2} E_{1}^{2} E_{2}^{-1}, \gamma=E_{2}^{-1} E_{3}^{-2} E_{2}$.

To find $\left[E_{1}^{2}, \gamma\right]$ we apply on $\left[E_{1}, E_{2}^{-1} E_{3} E_{2}\right]=1$ the Invariance Theorem (Corollary I.5) with $\rho_{1}\left(m_{1}=1\right.$ and all other $\left.m_{i}=0\right)$ to get $\left[E_{1}^{\prime}, E_{2}^{-1} E_{3} E_{2}\right]=1$ and thus, we get $\left[E_{1^{\prime}}^{2}, E_{2}^{-1} E_{3}^{2} E_{2}\right]=1$. i.e., $\left[E_{1^{\prime}}^{2}, \gamma\right]=1$.

$\left[E_{1^{\prime}}^{2}, \alpha\right]=\left[E_{1^{\prime}}^{2}, E_{3}^{2}\right]=\left[\left(E_{1}^{2}\right) \rho_{1}, E_{3}^{2}\right]=\left(\left[E_{1}^{2}, E_{3}^{2}\right]\right) \rho_{1}=(\mu) \rho_{1}=$ $\mu$ (By IV.5.1).

$$
\begin{aligned}
{\left[E_{1^{\prime}}^{2}, \beta\right]=\left[E_{1^{\prime}}^{2}, E_{2} E_{1}^{2} E_{2}^{-1}\right] } & =\left[E_{1^{\prime}}^{2}, E_{1}^{-1} E_{2}^{2} E_{1}\right]=\text { (Claim II.4) } \\
& =\left[E_{1^{\prime}}^{2}, E_{1} \cdot E_{1}^{-2} E_{2}^{2} E_{1}^{2} E_{2}^{-2} \cdot E_{2}^{2} E_{1}^{-1}\right] \\
& =\left[E_{1^{\prime}}^{2}, E_{1} \cdot \mu \cdot E_{2}^{2} E_{1}^{-1}\right]=(\mu \in \operatorname{Center}(G)) \\
& =\left[E_{1^{\prime}}^{2}, E_{1} E_{2}^{2} E_{1}^{-1}\right] \\
& =\left[E_{1}^{-1}, E_{1^{\prime}}^{2} E_{1}, E_{2}^{2}\right]_{E_{1}^{-1}} \\
& =\left[\left(E_{1}^{2}\right) \rho_{1}^{-1}, E_{2}^{2}\right]_{E_{1}^{-1}} \\
& =\left(\left[E_{1}^{2}, E_{2}^{2}\right] \rho_{1}^{-1}\right)_{E_{1}^{-1}} \\
& =\left((\mu) \rho_{1}^{-1}\right)_{E_{1}^{-1}} \quad(\operatorname{Lemma} \text { IV.5.1) } \\
& =\mu_{E_{1}^{-1}}=(\mu \in \operatorname{Center}(G)) \\
& =\mu .
\end{aligned}
$$

To find $\left[E_{1^{\prime}}^{2}, \alpha \beta \gamma\right]$ we use Claim II.4(d),

$$
\begin{aligned}
{\left[E_{1^{\prime}}^{2}, E_{1}^{2}\right]=\left[E_{1^{\prime}}^{2}, \alpha \beta \gamma\right] } & =\left[E_{1^{\prime}}^{2}, \alpha\right]\left[E_{1^{\prime}}^{2}, \beta\right]_{\alpha^{-1}} \cdot\left[E_{1^{\prime}}^{2}, \gamma\right]_{\beta^{-1} \gamma^{-1}} \\
& =\mu \cdot(\mu)_{\alpha} \cdot(1)_{z}=(\operatorname{since} \mu \in \operatorname{Center}(G)) \\
& =\mu \cdot \mu \cdot 1=\quad\left(\text { since } \mu^{2}=1\right) \\
& =1 .
\end{aligned}
$$


(2b) Let $B=(A)_{\tilde{X}_{2}^{-1}}=(A)_{\tilde{T}_{3}^{-1}}=(A)_{E_{3}^{-1}}$. To prove $(2 \mathrm{~b})$ we have to show $\nu_{B}=\nu_{E_{1}}^{-1}$. Now:

$$
\begin{aligned}
& \nu_{B}=\nu_{(A)_{E_{3}^{-1}}} \\
& =A_{E_{3}^{-1}}^{-1} \cdot \nu \cdot A_{E_{3}^{-1}} \\
& =E_{3} E_{1} \underbrace{E_{1^{\prime}}^{-1} E_{3}^{-1} \cdot E_{1^{\prime}}^{2}} E_{1}^{-2} \cdot E_{3} E_{1^{\prime}} E_{1}^{-1} E_{3}^{-1}= \\
& \text { (By Claim II.4(a)) } \\
& =\underbrace{E_{3} E_{1} E_{3}^{2}} E_{1^{\prime}}^{-1} \underbrace{E_{3}^{-1} E_{1}^{-2} E_{3}} E_{1^{\prime}} E_{1}^{-1} E_{3}^{-1}= \\
& \text { (By Claim II.4(a)) } \\
& =E_{1}^{2} E_{3} E_{1} E_{1^{\prime}}^{-1} \overbrace{E_{1} E_{3}^{-2} E_{1}^{-1}} E_{1^{\prime}} E_{1}^{-1} E_{3}^{-1} \\
& =E_{1}^{2} E_{3} E_{1}^{2} \underbrace{E_{1}^{-1} E_{1^{\prime}}^{-1} E_{1}} E_{3}^{-2} \underbrace{E_{1}^{-1} E_{1^{\prime}} E_{1}} E_{1}^{-2} E_{3}^{-1} \\
& =E_{1}^{2} E_{3} E_{1}^{2} \underbrace{\left(E_{1}\right) \rho_{1}^{-1} \cdot E_{3}^{-2}\left(E_{1}\right) \rho_{1}^{-1}} E_{1}^{-2} E_{3}^{-1}= \\
& \left(\begin{array}{c}
\text { By Claim II.4(a) } \\
\text { and Prop. II.6 (1) }
\end{array}\right) \\
& =E_{1}^{2} E_{3} E_{1}^{-2} \overbrace{E_{3}\left(E_{1}^{2}\right) \rho_{1}^{-1} E_{3}^{-1}} E_{1}^{-2} E_{3}^{-1} \\
& =E_{1}^{2} \cdot \underbrace{E_{3} E_{1}^{2} E_{3}^{-1}} \cdot \underbrace{E_{3}^{2}\left(E_{1}^{2}\right) \rho_{1}^{-1} E_{3}^{-2}} \cdot \underbrace{E_{3} E_{1}^{-2} E_{3}^{-1}}= \\
& \left(\begin{array}{c}
\text { By Claim II.4(a) and } \\
\text { Lemmas IV.3.4, IV.4.1 }
\end{array}\right) \\
& =E_{1}^{2} \cdot \overbrace{E_{1}^{-1} E_{3}^{2} E_{1}} \overbrace{\mu \cdot\left(E_{1}^{2}\right) \rho_{1}^{-1}} \cdot \overbrace{E_{1}^{-1} E_{3}^{-2} E_{1}}= \\
& (\mu \in \operatorname{Center}(G)) \\
& =\mu E_{1}^{2} \cdot E_{1}^{-1} E_{3}^{2} E_{1} \cdot E_{1}^{-1} E_{1^{\prime}}^{-2} E_{1} \cdot E_{1}^{-1} E_{3}^{-2} E_{1} \\
& =\mu E_{1} E_{3}^{2} E_{1^{\prime}}^{-2} E_{3}^{-2} E_{1} \\
& =\mu E_{1} \mu E_{1^{\prime}}^{-2} \cdot E_{1}= \\
& \text { (Since } \mu^{2}, \mu \in \operatorname{Center}(G) \text { ) } \\
& =E_{1} E_{1^{\prime}}^{-2} E_{1} \\
& \text { Thus, } \nu_{B}=E_{1} E_{1^{\prime}}^{-2} E_{1} \text {. }
\end{aligned}
$$

On the other hand, $\nu_{E_{1}}^{-1}=E_{1}^{-1}\left(E_{1^{\prime}}^{2} E_{1}^{-2}\right)^{-1} E_{1}=E_{1} E_{1^{\prime}}^{-2} E_{1}$. 
(3) We have to prove $A_{\tilde{X}_{j}}=A \quad \forall j \geq 3$. We recall: $A=E_{1^{\prime}} E_{1}^{-1}$ and $A_{X_{j}}=A_{\alpha\left(X_{j}\right)}$. Since $X_{3}=\left(T_{5}\right)_{T_{9}}, X_{4}=T_{9}, X_{5}=T_{8}, X_{6}=T_{7}$ and $X_{7}=T_{6}$, we get $\alpha\left(X_{j}\right)$ for $j \geq 3$ is a product of $E_{i}$ for $i=5,9,8,7,6$. $\left[E_{1}, E_{i}\right]=\left[E_{1^{\prime}}, E_{i}\right]=1$ for $i=5,9,8,7,6$ (Proposition II.6), we get $A_{\tilde{X}_{j}}=A_{\alpha\left(\tilde{X}_{j}\right)}=A \forall j=3,4,5,6,7$. Now: $\tilde{X}_{8}=\left(\tilde{T}_{2}\right)_{\tilde{T}_{3}^{-1}} \tilde{T}_{5}^{-1} \tilde{T}_{8}^{-1} \tilde{T}_{7}^{-1} \tilde{T}_{6}^{-1}$. Thus, $\alpha\left(\tilde{X}_{8}\right)=\left(E_{2}\right)_{E_{3}^{-1} E_{5}^{-1} E_{8}^{-1} E_{7}^{-1} E_{6}^{\prime-1}}$. Thus, $A_{\tilde{X}_{8}} \stackrel{A_{\alpha\left(\tilde{X}_{8}\right)}}{=} A_{\left(E_{2}\right)} E_{3}^{-1} E_{5}^{-1} E_{8}^{-1} E_{7}^{-1} E_{6}^{-1}$.

We first prove that $(A)_{\left(E_{2}\right)_{E_{3}^{-1}}}=A$.

Since $\left(T_{2}\right)_{T_{3}^{-1}}$ and $T_{1}$ are disjoint $\left[T_{1},\left(T_{2}\right)_{T_{3}^{-1}}\right]=1$. Thus

$$
\alpha\left(\left[\tilde{T}_{1},\left(\tilde{T}_{2}\right)_{\tilde{T}_{3}^{-1}}\right]\right)=1
$$

Thus, $\left[E_{1},\left(E_{2}\right)_{E_{3}^{-1}}\right]=1$. We apply on this relation the invariance Theorem (Corollary I.5) to get $\left[E_{1^{\prime}},\left(E_{2}\right)_{E_{3}^{-1}}\right]=1$. Since $A=E_{1^{\prime}} E_{1}^{-1}$ we get $\left[A,\left(E_{2}\right)_{E_{3}^{-1}}\right]=1$. Thus, $A_{\left(E_{2}\right)}=A$. Thus, $A_{\tilde{X}_{8}^{-1}}=$

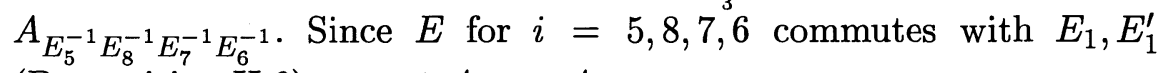
(Proposition II.6), we get $A_{\tilde{X}_{8}}=A$.

(4) Let $c=\left[\tilde{X}_{1}^{2}, \tilde{X}_{2}^{2}\right]$. We have to show that $A_{c}=A$. Since $c=\left[\tilde{X}_{1}^{2}, \tilde{X}_{2}^{2}\right]=$ $\left[\tilde{T}_{1}^{2}, \tilde{T}_{3}^{2}\right], \alpha(c)=\left[E_{1}^{2}, E_{3}^{2}\right]=\mu$, where $\mu \in \operatorname{Center}(G)$. Thus, $A_{c}=A_{\mu}=$ A.

Thus all the conditions of Proposition III.7.1 are fulfilled and $A$ is a prime element of $H_{1}$.

Proposition IV.7.3. A is a prime element of $G$ with s.h.t. $\tilde{T}_{1}$.

Proof. By the previous propositions, $A$ is a prime element of $H_{1}$ with s.h.t. $T_{1}$ and central element $\nu=A \cdot A_{\tilde{T}_{1}^{-1}}, \nu \in \operatorname{Center}\left(H_{1}\right)$. By the definition of a prime element, in order to prove that $A$ is also a prime element of $G$, it is enough to show that $\nu=A A_{\tilde{T}_{1}^{-1}} \in \operatorname{Center}(G)$. Since $A$ is a prime element of $H_{1}$ with central element $\nu$, we get $(\nu)_{b}=\nu \forall b \in \tilde{B}_{9}$. In particular, $(\nu)_{\tilde{T}_{i}}=\nu \forall i=1 \ldots 9$. Thus, $\nu_{E_{i}}=\nu \forall i=1 \ldots 9$. For $i \neq 1$, we apply $\rho$ on $\nu_{E_{i}}=\nu$, to get, using the Invariance Theorem, the relation $\nu_{E_{i}^{\prime}}=\nu$ (for $\left.i \neq 1(\nu) \rho_{i}=\nu\right)$. For $i=1$ we use $\left[\nu, E_{1}\right]=1$ (from above), and $[\nu, A]=1$ (since $A \in H_{1}$ and $\left.\nu \in \operatorname{Center}\left(H_{1}\right)\right)$ to get $\left[E_{1^{\prime}}, \nu\right]=\left[A E_{1}, \nu\right]=1$. Thus $\left[E_{i}, \nu\right]=\left[E_{i^{\prime}}, \nu\right]=1 \forall i=1 \ldots 9 \Rightarrow \nu \in \operatorname{Center}(G)$. 


\section{IV.8. New set of generators for $G\left\{\mathbf{E}_{\mathbf{j}}, \mathbf{h}_{\mathbf{j}}, \eta_{\mathbf{j}}\right\}_{\mathbf{j}=1}^{9}$.}

We introduce here a new set of generators for $G$. In $\S 3$ we introduced a homomorphism $\alpha: \tilde{B}_{9} \rightarrow G$ s.t. $\alpha\left(\tilde{T}_{i}\right)=E_{i}$ and introduced $G$ as a $\tilde{B}_{9}$ group using $\alpha$. In $\S 5$ we proved that $\alpha\left(\xi_{1}\right)=\eta_{1}=\left(E_{2}^{2}\right)_{E_{1}} \cdot E_{2}^{-2}$ is a prime element of $G$ with s.h.t. $\tilde{T}_{1}$ and central element $\mu=\left[E_{2}^{2}, E_{1}^{2}\right]$. We proved in $\S 7$ that $A=E_{1}, E_{1}$ is a prime element of $G$ with s.h.t. $\tilde{T}_{1}$ and central element $\nu=A_{E_{1}^{-1}}=E_{1^{\prime}}^{2} E_{1}^{-2}$. In $\S 6$ we introduced the $N$-situation. Here we consider the $N$-situation for $\alpha: \tilde{B}_{9} \rightarrow G$, and $h_{1}=A \in G$.

Consider the $N$-situation with $\eta_{i}, h_{i}, N_{1}, N_{2}$ as follows:

$h_{i}$ is the unique prime element with s.h.t. $\tilde{T}_{i}$ s.t. $\left(h_{i}, \tilde{T}_{i}\right)$ is coherent with $\left(A, \tilde{T}_{1}\right)$. The central element of $h_{i}$ is $\nu$.

$\eta_{i}$ is the unique prime element with s.h.t. $\tilde{T}_{i}$ s.t. $\left(\eta_{i}, \tilde{T}_{i}\right)$ is coherent with $\left(\eta_{1}, \tilde{T}_{1}\right)$. The corresponding central element is $\mu$.

$N_{1}=\left\{\eta_{i} \quad i=1 \ldots 0\right\}$.

$N_{2}=\left\{h_{i} \quad i=1 \ldots 0\right\}$.

Lemma IV.8.0. (i) Let $f$ be a prime element in $G$ with s.h.t. $\tilde{T}_{i}$. Then $f$ commutes with $E_{i}^{2}$.

(ii) If $T_{j}$ is transversal to $T_{i}$ then $f$ commutes with $E_{j}$.

(iii) If $T_{j}$ is consecutive to $T_{i}$ then $\left[\left(E_{i}\right)_{E_{j}^{ \pm 1}}, E_{i}^{2}\right]=\mu$.

Proof.

(i) By Lemma III.2.1, $(f)_{\tilde{T}_{i}^{2}}=f$. By definition of $G$ as a $\tilde{B}_{9 \text {-group: }}$ $(f)_{\tilde{T}_{i}^{2}}=f_{\alpha\left(\tilde{T}_{i^{\prime}}^{2}\right)}$. Since $\alpha\left(\tilde{T}_{i}\right)=E_{i} \quad$ (Lemma IV.3.4) we get $(f)_{E_{i}^{2}}=f$. Thus, $f$ commutes with $E_{i}^{2}$.

(ii) By Lemma III.4.2 $(f)_{\tilde{T}_{j}}=f$. But $f_{\alpha\left(\tilde{T}_{j}\right)}=f_{E_{j}}$. Thus it commutes with $E_{j}$.

(iii) Lemma IV.5.1.

Lemma IV.8.1. Let $A_{i}=E_{i}^{\prime} E_{i}^{-1}$. Let $h_{i}, \eta_{i} \mu, \nu$ be as above. Then,

(1) $A_{1}=A=h_{1}$.

(2) $A_{2}=h_{2}^{-1} \eta_{2}$.

(3) $A_{3}=h_{3} \eta_{3}^{-1} \mu \nu$.

(4) $A_{4}=h_{4}^{2} \eta_{4}^{-2} \mu \nu$. 
(5) $A_{5}=h_{5}^{2} \eta_{5}^{-2} \mu \nu$.

(6) $A_{6}=\eta_{6}^{3} h_{6}^{2} \mu \nu$.

(7) $A_{7}=h_{7} \eta_{7}^{-1} \mu \nu$.

(8) $A_{8}=h_{8}^{-1} \eta_{8}$

(9) $A_{9}=h_{9}^{2} \eta_{9}^{-3} \nu$.

Proof. We use the definition of prime element and Lemmas II.7, IV.5.3, IV.6.1 IV.6.2, IV.6.3, and IV.8.0. Recall that $\nu^{2}=\mu^{2}=1, \nu, \mu \in \operatorname{Center}(G)$. We use here often the following two facts: If $f$ is a prime element in $G$ with s.h.t. $\tilde{T}_{i}$, then $f$ commutes with $E_{i}^{2}$ (Lemma IV.8.0(i)); $\left(E_{i}^{2}\right)_{E_{j}^{ \pm 1}}$ and $E_{i}^{2}$ commute up to $\mu$ for $T_{i}$ and $T_{j}$ consecutive half-twists (Lemma IV.8.0(iii)).

(1) By definition of $h_{1}$.

(2) By Corollary II.7, $A_{2}=E_{1}^{-2} A_{1}^{-1}\left(A_{1}\right)_{E_{2}^{-1}}\left(E_{1}^{2}\right)_{E_{2}^{-1}}$. Since $A_{1}$ is a prime element with s.h.t. $\tilde{T}_{1}$ (Proposition IV.7.3), $A_{1}$ commutes with $E_{1}^{2}$. Thus, $A_{2}=A_{1}^{-1} E_{1}^{-2}\left(E_{1}^{2}\right)_{E_{2}^{-1}}\left(A_{1}\right)_{E_{2}^{-1}} A_{1}^{-1}=h_{1}^{-1}$ from (1). $E_{1}^{-2}\left(E_{1}^{2}\right)_{E_{2}^{-1}}=\eta_{2}$ by Lemma IV.5.3. $\quad\left(A_{1}\right)_{E_{2}^{-1}}=\left(h_{1}\right)_{\tilde{T}_{2}^{-1}}=h_{2}^{-1} h_{1}$ since $\tilde{T}_{1}$ and $\tilde{T}_{2}$ are not orderly adjacent (by Lemma IV.6.3).

Thus, $A_{2}=h_{1}^{-1} \eta_{2} h_{2}^{-1} h_{1}$. By Lemma IV.6.2, $\eta_{2}$ commutes with $h_{2}^{-1}$ and $\eta_{2} h_{1}=h_{1} \eta_{2} \nu(\nu \in \operatorname{Center}(G))$. Thus, $A_{2}=\nu h_{1}^{-1} h_{2}^{-1} h_{1} \eta_{2}$. By the same Lemma, $h_{1}^{-1} h_{2}^{-1} h_{1}=\nu h_{2}^{-1}$. Thus, $A_{2}=\nu^{2} h_{2}^{-1} \eta_{2}=h_{2}^{-1} \eta_{2}$.

(3) By Corollary II.7, $A_{3}=E_{1}^{-2} A_{1}^{-1}\left(A_{1}\right)_{E_{3}^{-1}}\left(E_{1}^{2}\right)_{E_{3}^{-1}}$. Like in (2) we can write, $A_{3}=A_{1}^{-1} E_{1}^{-2}\left(E_{1}^{2}\right)_{E_{3}^{-1}}\left(A_{1}\right)_{E_{3}^{-1}}$.

$A_{1}^{-1}=h_{1}^{-1}$ from (1). $E_{1}^{-2}\left(E_{1}^{2}\right)_{E_{3}^{-1}}=\eta_{3}^{-1} \mu$. (Lemmas IV.5.3 and IV.5.1(d)). $\quad\left(A_{1}\right)_{E_{3}^{-1}}=\left(h_{1}\right)_{\tilde{T}_{3}^{-1}}=h_{1} h_{3}$. (Lemma IV.6.3). Thus, $A_{3}=h_{1}^{-1} \mu \eta_{3}^{-1} h_{1} h_{3}$. Since $\left[h_{1}^{-1}, \eta_{3}^{-1}\right]=\nu$, and $\mu \in \operatorname{Center}(G)$, $A_{3}=\mu \eta_{3}^{-1} h_{1}^{-1} \nu h_{1} h_{3}$.

Since $\mu, \nu \in \operatorname{Center}(G), A_{3}=\mu \nu \eta_{3}^{-1} h_{3}^{-1}$ Since $\eta_{3}$ commutes with $h_{3}$, $A_{3}=h_{3} \eta_{3}^{-1} \mu \nu$.

(4) By Corollary II.7,

$$
\left(A_{4}\right)_{E_{2}^{-1} E_{4}^{-1}}=E_{4}^{2} A_{3} E_{3}^{2} A_{2}\left(E_{3}^{-2}\right)_{E_{2}^{-1}}\left(A_{3}^{-1}\right)_{E_{2}^{-1}}\left(E_{4}^{-2}\right)_{E_{2}^{-1}}
$$




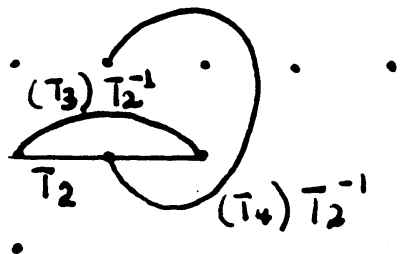

Figure IV.8.

Since $A_{3}$ is a product of prime elements with s.h.t. $\tilde{T}_{3}, A_{3}$ commutes with $E_{3}^{2}\left(=\alpha\left(\tilde{T}_{3}^{2}\right)\right)$ (Lemma IV.8.0). Thus,

$$
\left(A_{4}\right)_{E_{2}^{-1} E_{4}^{-1}}=E_{4}^{2} E_{3}^{2} A_{3} A_{2}\left(E_{3}^{-2}\right)_{E_{2}^{-1}}\left(A_{3}^{-1}\right)_{E_{2}^{-1}}\left(E_{4}^{-2}\right)_{E_{2}^{-1}}
$$

Now, $E_{4}=\alpha\left(\tilde{T}_{4}\right), \tilde{T}_{4}$ is a half-twist which is transversal to $\tilde{T}_{3}$. Thus, by Corollary IV.8.0(ii), $E_{4}^{-2}$ commutes with $A_{3}^{-1}$. Thus, $\left(A_{4}\right)_{E_{2}^{-1}, E_{4}^{-1}}=$ $E_{1}^{2} E_{3}^{2} A_{3} A_{2}\left(E_{3}^{-2}\right)_{E_{2}^{-1}}\left(E_{4}^{-2}\right)_{E_{2}^{-1}}\left(A_{3}^{-1}\right)_{E_{2}^{-1}} . Z_{1}=\left(T_{3}\right)_{T_{2}^{-1}}$ and $Z_{2}=$ $\left(T_{4}\right)_{T_{2}^{-1}}$ are 2 half-twists which are adjacent to $T_{3}$ and to $T_{2}$ (Fig. IV.8). Thus, by Lemma IV.6.1, $\left[\alpha\left(Z_{i}^{2}\right), f\right]=\nu$ where $f$ is a prime element with s.h.t. $T_{3}$ or $T_{2}$ and central element $\nu$ and $\left[\alpha\left(Z_{i}^{2}\right), \eta\right]=\mu$ where $\eta$ is a prime element with s.h.t. $T_{3}$ or $T_{2}$ and central element $\mu$ $(i=1,2)$.

$A_{3} A_{2}$ is a product of 4 prime elements with s.h.t. $\tilde{T}_{2}$ or $\tilde{T}_{3}$. Two of them have a central element $\mu$, and 2 of them have a central element $\nu$. Thus, $A_{3} A_{2} \alpha\left(Z_{1}^{-2}\right) \alpha\left(Z_{2}^{-2}\right)=(\nu \mu)^{2} \alpha\left(Z_{1}^{-2}\right) \alpha\left(Z_{2}^{-2}\right) A_{3} A_{2}$. Since $\alpha\left(Z_{1}\right)=\left(E_{3}\right)_{E_{2}^{-1}}, \alpha\left(Z_{2}\right)=\left(E_{4}\right)_{E_{2}^{-1}}$ and $\mu^{2}=\nu^{2}=1$, we have $A_{3} A_{2}\left(E_{3}^{-2}\right)_{E_{2}^{-1}}\left(E_{4}^{-2}\right)_{E_{2}^{-1}}=\left(E_{3}^{-2}\right)_{E_{2}^{-1}}\left(E_{4}^{-2}\right)_{E_{2}^{-1}} \cdot A_{3} A_{2}$. Thus, $\left(A_{4}\right)_{E_{2}^{-1} E_{4}^{-1}}=E_{4}^{2} E_{3}^{2}\left(E_{3}^{-2}\right)_{E_{2}^{-1}}\left(E_{4}^{-2}\right)_{E_{2}^{-1}} A_{3} A_{2}\left(A_{3}^{-1}\right)_{E_{2}^{-1}}$.

From Corollary IV.4.2, $\tilde{T}_{4}^{2}\left(\tilde{T}_{4}^{2}\right)_{\tilde{T}_{2}^{-1}}^{-2}=\tilde{T}_{3}^{-2}\left(\tilde{T}_{3}^{2}\right)_{\tilde{T}_{2}^{-1}}$. We use Lemma IV.4.1(4) and $c^{2}=1$ to exchange factors and rewrite this equation as $\tilde{T}_{4}^{2} \tilde{T}_{3}^{2}\left(\tilde{T}_{3}^{-2}\right)_{\tilde{T}_{2}^{-1}}\left(\tilde{T}_{4}^{-2}\right)_{\tilde{T}^{-1}}=1$. We apply $\alpha$ to it to get: $E_{4}^{2} E_{3}^{2}\left(E_{3}^{-2}\right)_{E_{2}^{-1}}\left(E_{4}^{-2}\right)_{E_{2}^{-1}}=1$. Thus, $\left(A_{4}\right)_{E_{2}^{-1} E_{4}^{-1}}=A_{3} A_{2}\left(A_{3}^{-1}\right)_{E_{2}^{-1}}$. By (2) (3),

$$
\begin{aligned}
\left(A_{4}\right)_{E_{2}^{-1} E_{4}^{-1}} & =h_{3} \eta_{3}^{-1} \mu \nu h_{2}^{-1} \eta_{2}\left(\eta_{3} h_{3}^{-1}\right)_{\tilde{T}_{2}^{-1}} \mu \nu \\
& =h_{3} \eta_{3}^{-1} h_{2}^{-1} \eta_{2} \eta_{3} \eta_{2} h_{2}^{-1} h_{3}^{-1} .(\text { By Lemma IV.6.3) }
\end{aligned}
$$

Since $\left[h_{2}^{-1}, \eta_{3}^{-1}\right]=\nu$ and $\left[\eta_{2}, \eta_{3}^{-1}\right]=\mu$ (Lemma IV.6.2), we get $\left(A_{4}\right)_{E_{2}^{-1} E_{4}^{-1}}=\mu \nu h_{3} h_{2}^{-1} \eta_{2}^{2} h_{2}^{-1} h_{3}^{-1}$. Since $h_{2}$ commutes with $\eta_{2}$ and 
$\left[h_{3}, h_{2}^{-1}\right]=\left[h_{3}, \eta_{2}\right]=\nu$, we get $\left(A_{4}\right)_{E_{2}^{-1} E_{4}^{-1}}=\nu^{5} \mu \eta_{2}^{2} h_{2}^{-2}=\nu \mu \eta_{2}^{2} h_{2}^{-2}$.

Thus,

$$
A_{4}=\left(\nu \mu \eta_{2}^{2} h_{2}^{-2}\right)_{E_{4} E_{2}}
$$

Since

$$
\begin{aligned}
& \left(\eta_{2}\right)_{E_{2}}=\eta_{2}^{-1} \mu \\
& \left(h_{2}\right)_{E_{2}}=h_{2}^{-1} \nu \\
& \left(h_{4}\right)_{E_{2}}=h_{4} h_{2}^{-1} \\
& \left(\eta_{4}\right)_{E_{2}}=\eta_{4} \eta_{2}^{-1}
\end{aligned}
$$

So,

$$
\begin{aligned}
A_{4} & =\nu \mu\left(\eta_{2}^{-1} \mu \eta_{2} \eta_{4}^{-1}\right)^{2} \circ\left(h_{2}^{-1} \nu h_{2} h_{4}^{-1}\right)^{-2} \\
& =\nu \mu \eta_{4}^{-2} h_{4}^{+2} .
\end{aligned}
$$

(5) By Corollay II.7.

$$
\begin{aligned}
A_{5} & =\left(A_{4}\right)_{E_{2}^{-1} E_{3} E_{7}^{-1} E_{8}}=(\text { from (4)) } \\
& =\nu \mu\left(\eta_{4}\right)_{E_{2}^{-1} E_{3} E_{7}^{-1} E_{8}}\left(h_{4}\right)_{E_{2}^{-1} E_{3} E_{7}^{-1} E_{8}}^{2} .
\end{aligned}
$$

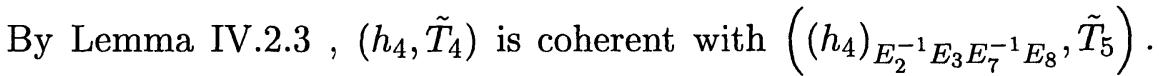

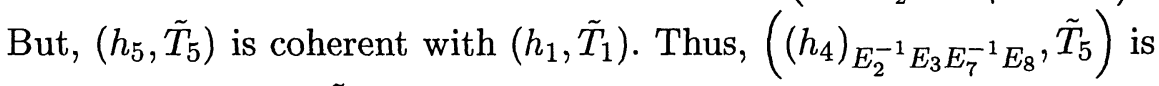

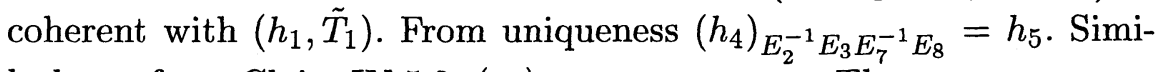
larly, or from Claim IV.5.3, $\left(\eta_{4}\right)_{E_{2}^{-1} E_{3} E_{7}^{-1} E_{8}}=\eta_{5}$. Thus,

$$
A_{5}=\nu \mu \eta_{5}^{-2} h_{5}^{+2} \text {. }
$$

(6) By Corollary II.7,

$$
A_{6}=E_{4}^{-2} A_{4}^{-1}\left(A_{4}\right)_{E_{6}^{-1}}\left(E_{4}^{2}\right)_{E_{6}^{-1}} .
$$

By (4) above, $A_{4}$ is a product of prime elements, with s.h.t. $\tilde{T}_{4}$. Thus, $A_{4}$ commutes with $E_{1}^{2}$. Thus:

$$
A_{6}=A_{4}^{-1} E_{4}^{-2}\left(E_{4}^{2}\right)_{E_{6}^{-1}}\left(A_{4}\right)_{E_{6}^{-1}} .
$$


By Lemma IV.5.3, $E_{4}^{-2}\left(E_{4}^{2}\right)_{E_{6}^{-1}}=\eta_{6}$. Thus,

$$
A_{6}=\nu \mu h_{4}^{+2} \eta_{4}^{-2} \eta_{6} \nu \mu\left(\eta_{4}^{-2}\right)_{E_{6}^{-1}}\left(h_{4}^{+2}\right)_{E_{6}^{-1}}
$$

Since $\tilde{T}_{4}$ and $\tilde{T}_{6}$ are not orderly adjacent,

$$
\begin{aligned}
\left(\eta_{4}\right)_{E_{6}^{-1}}=\left(\eta_{4}\right)_{\tilde{T}_{6}^{-1}} & =\eta_{6}^{-1} \eta_{4} \\
\left(h_{4}\right)_{E_{6}^{-1}} & =h_{6}^{-1} h_{4} .
\end{aligned}
$$

Thus,

$$
\begin{aligned}
A_{6} & =h_{4}^{-2} \eta_{4}^{+2} \eta_{6}\left(\eta_{6}^{-1} \eta_{4}\right)^{-2}\left(h_{6}^{-1} h_{4}\right)^{2} \\
& =h_{4}^{-2} \eta_{4}^{+2} \eta_{6} \eta_{4}^{-1} \eta_{6} \eta_{4}^{-1} \eta_{6} h_{6}^{-1} h_{4} h_{6}^{-1} h_{4} .
\end{aligned}
$$

Since $T_{4}$ and $T_{6}^{-1}$ are adjacent, $\left[h_{4}, \eta_{6}\right]=\left[h_{4}, h_{6}\right]=\nu$ (Lemma IV.6.2), and thus, $h_{4} h_{6}^{-1}=h_{6}^{-1} h_{4} \nu$ and $\eta_{6} h_{4}=h_{4} \eta_{6} \nu$. Moreover, $h_{4}$ and $\eta_{4}$ commute. Thus,

$$
A_{6}=\nu^{9} \eta_{4}^{+2} \eta_{6} \eta_{4}^{-1} \eta_{6} \eta_{4}^{-1} \eta_{6} h_{6}^{-2} .
$$

Since $\left[\eta_{6}, \eta_{4}\right]=\mu, \eta_{6} \eta_{4}^{-1}=\eta_{4}^{-1} \eta_{6} \mu$, and thus, $A_{6}=\nu^{9} \mu^{3} \eta_{6}^{3} h_{6}^{-2}=$ $\eta_{5}^{3} h_{6}^{-2} \nu \mu$.

(7) and (8): Similar to the proof of (5).

(9) By Corollary II.7,

$$
A_{9}=E_{5}^{-2} A_{5}^{-1}\left(A_{5}\right)_{E_{9}^{-1}}\left(E_{5}^{2}\right)_{E_{9}^{-1}}
$$

Since $A_{5}$ is a product of prime elements in $G$ with s.h.t. $\tilde{T}_{5}$, it commutes with $E_{5}^{2}$. Thus,

$$
A_{9}=A_{5}^{-1} E_{5}^{-2}\left(E_{5}^{2}\right)_{E_{9}^{-1}}\left(A_{5}\right)_{E_{9}^{-1}} .
$$

By Lemma IV.5.3, $E_{5}^{-2}\left(E_{5}^{+2}\right)_{E_{9}^{-1}}=\mu \eta_{9}^{-1}$. Thus,

$$
A_{9}=\nu \mu h_{5}^{-2} \eta_{5}^{2} \cdot \mu \eta_{9}^{-1} \cdot\left(\nu \mu \eta_{5}^{-2} h_{5}^{+2}\right)_{E_{9}^{-1}} .
$$

Since $T_{5}$ and $T_{9}$ are orderly adjacent,

$$
\begin{aligned}
\left(\eta_{5}\right)_{E_{9}^{-1}}=\left(\eta_{5}\right)_{\tilde{T}_{9}^{-1}} & =\eta_{5} \eta_{9} \\
\left(h_{5}\right)_{E_{9}^{-1}} & =h_{5} h_{9} .
\end{aligned}
$$


Thus,

$$
A_{9}=h_{5}^{-2} \eta_{5}^{2} \mu \eta_{9}^{-1}\left(\eta_{5} \eta_{9}\right)^{-2}\left(h_{5} h_{9}\right)^{2} .
$$

Since $\left[\eta_{5}, \eta_{9}\right]=\mu$, then $\eta_{9}^{-1} \eta_{5}^{-1}=\eta_{5}^{-1} \eta_{9}^{-1} \mu$. Since $\left[h_{5}, h_{9}\right]=\left[h_{5}, \eta_{9}\right]=$ $\nu$, then $h_{9} h_{5}=h_{5} h_{9}^{-1} \nu$ and $\eta_{9}^{-1} h_{5}=h_{5} \eta_{9}^{-1} \nu$. Thus we can collect all $h_{5}$ and $\eta_{5}$ at the left to get

$$
A_{9}=h_{5}^{-2} \eta_{5}^{2} \eta_{5}^{-2} h_{5}^{2} \eta_{9}^{-1} \eta_{9}^{-2} h_{9}^{2} \cdot \mu^{4} \nu^{3} .
$$

Thus, $A_{9}=\eta_{9}^{-3} h_{9}^{2} \nu$.

Lemma IV.8.2. (a) $h_{i}^{3}=\eta_{i}^{3} \quad \forall i=1 \ldots 9$.

(b) $\nu \mu=1$.

Proof. For $T_{i}$ and $T_{j}$ orderly adjacent

$$
\begin{aligned}
\left(h_{i}\right)_{E_{j}^{-1} E_{i}^{-1}} & =\left(h_{i} h_{j}\right)_{E_{i}^{-1}} \\
& =\nu h_{i}^{-1} h_{j} h_{i} \quad(\text { By Lemma IV.6.3) } \\
& =\nu h_{i}^{-1} \nu h_{i} h_{j} \quad(\text { By Lemma IV.6.2) } \\
& =h_{j} \quad\left(\text { Since } \nu^{2}=1, \nu \in \operatorname{Center}(G)\right) .
\end{aligned}
$$

Similarly,

$$
\left(\eta_{i}\right)_{E_{j}^{-1} E_{i}^{-1}}=\eta_{j}
$$

Thus

$$
\begin{aligned}
& \left(h_{j}\right)_{E_{i} E_{j}}=h_{i} \\
& \left(\eta_{j}\right)_{E_{i} E_{j}}=\eta_{i} .
\end{aligned}
$$

(a) By Corollary II.7,

$$
\left(A_{9}\right)_{E_{8}^{-1} E_{9}^{-1}}=E_{9}^{2} A_{8}\left(E_{9}^{-2}\right)_{E_{8}^{-1}}=E_{9}^{2} h_{8}^{-1} \eta_{8}\left(E_{9}^{-2}\right)_{E_{8}^{-1}}
$$

Since $T_{9}$ is a half-twist adjacent to $T_{8}$ and $h_{8}, \eta_{8}$ are prime elements with s.h.t. $\tilde{T}_{8}$, we get by Lemma IV.6.1 that $E_{9}^{2} h_{8}^{-1}=\nu h_{8}^{-1} E_{9}^{2}$ and $E_{9}^{2} \eta_{8}=\mu \eta_{8} E_{9}^{2}$. Thus,

$$
\left(A_{9}\right)_{E_{8}^{-1} E_{9}^{-1}}=E_{9}^{2} A_{8}\left(E_{9}^{-2}\right)_{E_{8}^{-1}}=\nu \mu h_{8}^{-1} \eta_{8} E_{9}^{2}\left(E_{9}^{-2}\right)_{E_{8}^{-1}} .
$$


By Lemmas IV.5.3 and IV.8.0 $E_{9}^{2}\left(E_{9}^{-2}\right)_{E_{8}^{-1}}=\mu \eta_{8}^{-1}$. Thus,

$$
E_{9}^{2} A_{8}\left(E_{9}^{-2}\right)_{E_{8}^{-1}}=\nu h_{8}^{-1} \text {. }
$$

Thus, $\left(A_{9}\right)_{E_{8}^{-1} E_{9}^{-1}}=\nu h_{8}^{-1}$ and $A_{9}=\nu\left(h_{8}^{-1}\right)_{E_{9} E_{8}}=\nu h_{9}^{-1}$. We compare this with the previous Lemma to get $\eta_{9}^{-3} h_{9}^{2} \nu=h_{9}^{-1} \nu$. Thus, $\eta_{9}^{-3} h_{9}^{3}=1$. Since $\left(\eta_{9}, \tilde{T}_{9}\right)$ is coherent with $\left(\eta_{i}, \tilde{T}_{i}\right)$ and $\left(h_{9}, \tilde{T}_{9}\right)$ is coherent with $\left(h_{i}, \tilde{T}_{i}\right)$, we can use Corollary III.3.5 to conclude that $\forall i \exists \tilde{B}_{i} \in \tilde{B}_{9}$ s.t. $\eta_{i}=\left(\eta_{9}\right)_{\tilde{B}_{i}}$ and $h_{i}=\left(h_{9}\right)_{\tilde{B}_{i}}$. Thus, $\eta_{i}^{-3} h_{i}^{3}=1 \quad \forall i$.

(b) By Corollary II.7, $\left(A_{9}\right)_{E_{8}^{-1} E_{9}^{-1}}=E_{9}^{2} A_{8}\left(E_{9}^{-2}\right)_{E_{8}^{-1}}$.

Thus,

$$
\begin{aligned}
& A_{8}=E_{9}^{-2}\left(A_{9}\right)_{E_{8}^{-1} E_{9}^{-1}}\left(E_{9}^{2}\right)_{E_{8}^{-1}}=
\end{aligned}
$$

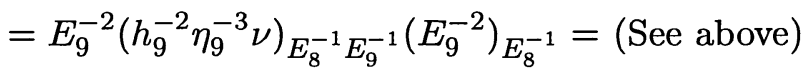

$$
\begin{aligned}
& =E_{9}^{-2} h_{8}^{2} \eta_{8}^{-3} \nu\left(E_{9}^{2}\right)_{E_{8}^{-1}}= \\
& =E_{9}^{-2}\left(E_{9}^{2}\right)_{E_{8}^{-1}} h_{8}^{2} \eta_{8}^{-3} \nu^{3} \mu^{3}= \\
& =\eta_{8} h_{8}^{2} \eta_{8}^{-3} \nu \mu= \\
& =\eta_{8}^{-2} h_{8}^{2} \nu \mu \text {. }
\end{aligned}
$$

We compare with the previous result to get $\nu \mu=1$.

Proposition IV.8.3. Let $A_{i}=E_{i^{\prime}} E_{i}^{-1}$. Let $h_{i}, \eta_{i} \mu, \nu$ be as before. Then:
(1) $A_{1}=h_{1}$.
(2) $A_{2}=h_{2}^{-1} \eta_{2}$
(3) $A_{3}=h_{3} \eta_{3}^{-1}$.
(4) $A_{4}=h_{4}^{-1} \eta_{4}$.
(5) $A_{5}=h_{5}^{-1} \eta_{5}$.
(6) $A_{6}=h_{6}$.
(7) $A_{7}=h_{7} \eta_{7}^{-1}$.
(8) $A_{8}=h_{8}^{-1} \eta_{8}$. 


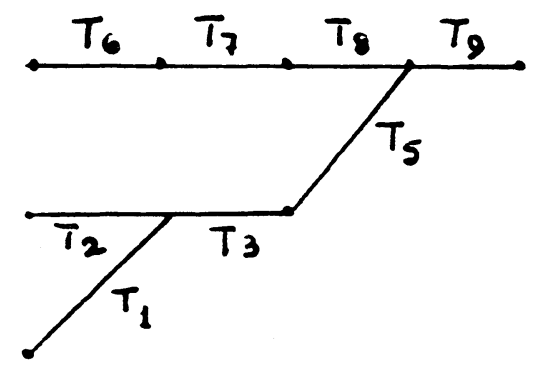

Figure V.1.

(9) $A_{9}=h_{9}^{-1} \nu$.

Proof. Immediately from the previous 2 lemmas.

Lemma IV.8.4. $\left\{E_{j}, \eta_{j}, h_{j}\right\}_{j=1}^{9}$ generates $G$.

Proof. By Lemma IV.7.1, $\left\{A_{j}, E_{j}\right\}$ generates $G$. By the previous lemma, $A_{j}$ is a product of $\nu, h_{j}, \eta_{j}$. However, $\nu$ is the central element of $\left(h_{1}, \tilde{T}_{1}\right)$. Thus, $\nu=\left(h_{1}\right)\left(h_{1}\right)_{\tilde{T}_{1}}$.

\section{Construction of $\mathbf{G}_{\mathbf{9}}, \quad \hat{\alpha}: \mathbf{G}_{\mathbf{9}} \stackrel{\sim}{\rightarrow} \mathbf{G}$.}

\section{Construction of $\mathrm{G}_{0}(9)$.}

Let $G_{0}(9)$ be the group generated by $g_{i} i=1 \ldots 9 i \neq 4$ with the following list of relations:

$$
\begin{aligned}
& {\left[g_{1}, g_{2}\right]^{2}=1 .} \\
& {\left[g_{1}, g_{2}\right] \in \operatorname{Center}\left(G_{0}(9) .\right)} \\
& {\left[g_{i}, g_{j}\right]= \begin{cases}{\left[g_{1}, g_{2}\right]} & T_{i}, T_{j} \text { are adjacent } \\
1 & T_{i}, T_{j} \text { are disjoint. }\end{cases} }
\end{aligned}
$$

where $T_{i}$ are described as follows:

Denote $\nu=\left[g_{1}, g_{2}\right]$. 
Let us reformulate the relations of $G_{0}(9)$ as follows:

$$
\begin{aligned}
G_{0}(9)=\left\langle g_{1}, \ldots, \check{g}_{4}, \ldots,\right. & g_{9} \mid\left[g_{i}, g_{j}\right] \\
& =\left\{\begin{array}{ll}
\tau & T_{i}, T_{j} \text { are adjacent, } \\
1 & T_{i}, T_{j} \text { are disjoint. }
\end{array} \tau^{2}=1 \quad \tau_{g_{0}}=\tau\right\rangle
\end{aligned}
$$

Remark. $G_{0}(9)$ or $G_{0}(n)$ in general can be described in a different way: Take $A_{n-1}$, a free abelian group on $n-1$ generators $A_{n-1}=\left\langle w_{1}, \ldots, w_{n-1}\right\rangle$. Define a skew-symmetric form on $A_{n-1}$ as follows:

$$
w_{i} \cdot w_{j}= \begin{cases}1 & |i-j|=1 \\ 0 & |i-j| \neq 1\end{cases}
$$

Let $G_{0}(n)$ be the unique central extension that satisfies

$$
1 \rightarrow \mathbb{Z} / 2 \stackrel{b}{\rightarrow} G_{0}(n) \stackrel{a}{\rightarrow} A_{n-1} \rightarrow 1
$$

where $G_{0}(n)$ is generated by $u_{1} \ldots u_{n-1}, a\left(u_{i}\right)=w_{i}$ and $\left[u_{i}, u_{j}\right]=$ $\begin{cases}1 & |i-j| \neq 1 \\ \tau & |i-j|=1\end{cases}$

and $b(1)=\tau$. We have: $\operatorname{Ab}\left(G_{0}(n)\right)=A_{n-1}, \quad G_{0}(n)^{\prime}=\{\tau, 1\} \simeq \mathbb{Z} / 2$.

\section{$\mathbf{G}_{0}(9)$ as a $\tilde{B}_{9}$-group.}

$$
\left(g_{i}\right)_{\tilde{T}_{k}}= \begin{cases}g_{i}^{-1} \tau & i=k \\ g_{i} & T_{i} \text { and } T_{k} \text { are disjoint } \\ g_{k} g_{i} & T_{i} \text { and } T_{k} \text { are orderly adjacent } \\ g_{i} g_{k}^{-1} & T_{i} \text { and } T_{k} \text { are not orderly adjacent }\end{cases}
$$

Remark V.0. Let $G_{0}(9)$ and $g_{i}$ be as above. Then $g_{i}$ is a prime element of $G_{0}(9)$ with s.h.t. $\tilde{T}_{i}$ and central element $\tau$.

Proof. By the actions of $\tilde{B}_{9}$ on $g_{i}$ and the axioms of prime element.

Consider the semidirect product: $\tilde{B}_{9} \rtimes G_{0}(9)$. 


\section{Construction of $\stackrel{\rtimes}{\alpha}: \tilde{\mathbf{B}}_{9} \rtimes \mathbf{G}_{0}(9) \rightarrow \mathbf{G}$.}

$$
\begin{aligned}
& \left.\stackrel{\rtimes}{\alpha}\right|_{\tilde{B}_{9}}=\alpha \\
& \quad \stackrel{x}{\alpha}\left(\tilde{T}_{i}\right)=E_{i} \\
& \quad \stackrel{\rtimes}{\alpha}\left(\xi_{i}\right)=\alpha\left(\xi_{i}\right)=\eta_{i} \text { (Lemma IV.5.3) } \\
& \stackrel{\rtimes}{\alpha}(c)=\alpha(c)=\mu \\
& \left.\stackrel{\rtimes}{\alpha}\right|_{G_{0}(9)} \text { defined by } \stackrel{\rtimes}{\alpha}\left(g_{i}\right)=h_{i} . \\
& \quad \stackrel{\rtimes}{\alpha}(\tau)=\stackrel{\rtimes}{\alpha}\left[g_{1}, g_{2}\right]=\left[h_{1}, h_{2}\right]=\nu .
\end{aligned}
$$

Since $\nu$ is the central element of $h_{i}$ it belong to $\operatorname{Center}(G)$ and is of order 2. Thus, by Proposition IV.4.2 all relations that $g_{i}$ satisfy are also satisfied by $h_{i}$, and thus $\left.\stackrel{\rtimes}{\alpha}\right|_{G_{0}(9)}$ is well-defined.

By Lemma IV.4.3, $\left.\hat{\alpha}\right|_{G_{0}(9)}$ is compatible with the action of $\tilde{B}_{9}$ on $G_{0}(9)$ and thus we have $\stackrel{\rtimes}{\alpha}: \tilde{B}_{9} \rtimes G_{0}(9) \rightarrow G$.

\section{Construction of $\mathbf{G}_{9}$.}

Let $g_{i}$ be the generators of $G_{0}(9)$ as above.

Let $\tau=\left[g_{1}, g_{2}\right]$.

Let $\xi_{i}$ be the prime elements in $\tilde{B}_{9}$ defined in Chapter IV.4 ( $\xi$-situation).

Let $c$ be the central element of $\xi_{i} \cdot c=\left[\tilde{T}_{1}^{2}, \tilde{T}_{2}^{2}\right]$.

Let $N_{9} \subseteq \tilde{B}_{a} \rtimes G_{0}(9)$ be the normal subgroup generated by $\tau c$ and $\left(g_{i} \xi_{i}\right)^{3}$ :

$N_{9}=\left\langle\left(\tau c^{-1}, \quad\left(g_{i} \xi_{i}^{-1}\right)^{3} i=1 \ldots 9 i \neq 4\right\rangle\right.$.

$G_{9}=\tilde{B}_{9} \rtimes G_{0}(9) / N_{9}$.

Construction of $\hat{\alpha}, \hat{\alpha}: \mathbf{G}_{\mathbf{9}} \rightarrow \mathbf{G}$.

By Lemma IV.4.5, $\nu \mu=1,\left(\eta_{i} \eta_{i}^{-1}\right)^{3}=1$, so $\stackrel{x}{\alpha}\left(N_{9}\right)=1$. Thus $\stackrel{x}{\alpha}$ induces a map on $G_{9}$ denoted by $\hat{\alpha}$. $\hat{\alpha}: G_{9} \rightarrow G$.

\section{Construction of $\hat{\beta}: \mathbf{G} \rightarrow \mathbf{G}_{\mathbf{9}}$.}

We start by using a set of generators for $G,\left\{\Gamma_{i}, \Gamma_{i^{\prime}}\right\}_{i=1}^{9}$.

We then choose as a set of generators $\left\{E_{i}, E_{i^{\prime}}\right\}_{i=1}^{9}$ where $E_{i}=$ $\left\{\begin{array}{ll}\Gamma_{i} & i \neq 2,7 \\ \Gamma_{i^{\prime}} & i=2,7\end{array}\right.$ and $E_{i^{\prime}}=\left(E_{i}\right) \varphi_{i}$. 
The third set of generators was $\left\{E_{i}, A_{i}\right\}_{i=1}^{9}$ where $A_{i}=E_{i^{\prime}} E_{i}^{-1}$. Finally, we take the following set of generators for $G: E_{i}, h_{i}, \eta_{i}$. We define $\hat{\beta}: G \rightarrow G_{9}$ on the third set of generators as follows: $\hat{\beta}\left(E_{i}\right)=\tilde{T}_{i} \quad i=1 \ldots 9$. $\hat{\beta}\left(A_{1}\right)=g_{1}$.

$\hat{\beta}\left(A_{2}\right)=g_{2}^{-1} \xi_{2}$.

$\hat{\beta}\left(A_{3}\right)=g_{3} \xi_{3}^{-1}$.

$\hat{\beta}\left(A_{4}\right)=g_{4}^{-1} \xi_{4}$.

$\hat{\beta}\left(A_{5}\right)=g_{5}^{-1} \xi_{5}$.

$\hat{\beta}\left(A_{6}\right)=g_{6}$.

$\hat{\beta}\left(A_{7}\right)=g_{7} \xi_{7}^{-1}$.

$\hat{\beta}\left(A_{8}\right)=g_{8}^{-1} \xi_{8}$.

$\hat{\beta}\left(A_{9}\right)=\tau g_{9}^{-1}$.

Remark. By definition of $\hat{\alpha}$ and by the formula for expressing $A_{i}$ in terms of $h_{i}$ and $\eta_{i}$ (Lemma IV.8.3), if $\hat{\beta}$ is well-defined, then $\hat{\alpha} \hat{\beta}=I d$.

Theorem V.1. $\hat{\beta}$ is well-defined.

Proof. We recall that $G=F_{18} / G(\varepsilon(18))$ where $F_{18}$ is the free group generated on $\left\{\Gamma_{i}, \Gamma_{i^{\prime}}\right\}_{i=1}^{9}$ and $G(\varepsilon(18))$ is the subgroup generated by the relations induced from the factors in the braid monodromy factorization $\varepsilon(18)$ (see Theorem I.1) by the Van Kampen method. To prove that $\hat{\beta}$ is well-defined, we have to prove that all relations induced by the Van Kampen Theorem are valid when each generator in a relation is replaced by its image under $\hat{\beta}$. In what follows we shall take every braid in the braid monodromy factorization $\varepsilon(18)$ and use the Van Kampen method to deduce from it a relation on $\pi_{1}\left(\mathbb{C P}^{2}-S\right)$ in terms of $\Gamma_{i}$ and $\Gamma_{i^{\prime}}$. Then we shall present the relation in terms of $E_{i}$ and $A_{i}$. The next step is to substitute $\tilde{T}_{i}$ instead of $E_{i}$ and $\hat{\beta}\left(A_{i}\right)$ instead of $A_{i}$ and confirm that the relation holds.

Denote:

$t_{i}=\hat{\beta}\left(E_{i}\right)=\tilde{T}_{i}$.

$t_{i^{\prime}}=\hat{\beta}\left(E_{i}^{\prime}\right)$.

$t_{i^{\prime \prime}}=\hat{\beta}\left(E_{i} E_{i^{\prime}} E_{i}^{-1}\right)$.

$a_{i}=\hat{\beta}\left(A_{i}\right)=\beta\left(E_{i^{\prime}} E_{i}^{-1}\right)=t_{i^{\prime}} t_{i}^{-1}$.

We have expressions for $a_{i}$ in terms of $g_{i}, \xi_{i}, \tau$.

$a_{1}=g_{1}$.

$a_{2}=g_{2}^{-1} \xi_{2}$.

$a_{3}=g_{3} \xi_{3}^{-1}$. 


$$
\begin{aligned}
& a_{4}=g_{4}^{-1} \xi_{4} . \\
& a_{5}=g_{5}^{-1} \xi_{5} . \\
& a_{6}=g_{6} . \\
& a_{7}=g_{7} \xi_{7}^{-1} . \\
& a_{8}=g_{8}^{-1} \xi_{8} . \\
& a_{9}=\tau g_{9}^{-1} .
\end{aligned}
$$

Then:

$$
\begin{aligned}
& t_{i}=\tilde{T}_{i} . \\
& t_{i^{\prime}}=a_{i} t_{i} . \\
& t_{i^{\prime \prime}}=\left(a_{i} t_{i}\right)_{t_{i}}=\left(a_{i}\right)_{t_{i}} \cdot t_{i}=t_{i}\left(a_{i}\right)_{t_{i}^{2}}=t_{i} a_{i} \text { (Lemma II.3). }
\end{aligned}
$$

$\varepsilon(18)$ implies relations of type $\left\langle b_{1}, b_{2}\right\rangle=1$ relations of type $\left[b_{1}, b_{2}\right]=1$ and relations of type $b_{1}=b_{2}$. We shall treat all relations of type $\left\langle b_{1}, b_{2}\right\rangle$ together. To do this we prove the following two lemmas:

Lemma 1. $\left(a_{i}\right)_{t_{i}}=\left(a_{i}\right)_{t_{i}^{-1}}= \begin{cases}\tau a_{i}^{-1} & i=1,6,9 \\ a_{i}^{-1} & \text { otherwise }\end{cases}$

Proof of Lemma 1. The elements $g_{i}$ and $\xi_{i}$ are prime elements with s.h.t. $t_{i}$. By Lemma II.5, for a prime element $g$ with s.h.t. $t$, we have:

$(g)_{t}=(g)_{t^{-1}}=\tau g^{-1}$.

For such $g$ we then also have:

$\left(g^{-1}\right)_{t}=\left(g^{-1}\right)_{t^{-1}}=\tau^{-1} g$.

Since $\tau^{-1}=\tau$ we have for every $f$ a prime element with s.h.t. $t$ or an inverse of a prime element with s.h.t. $t$ :

$(f)_{t}=(f)_{t^{-1}}=\tau f$.

For $i=1,6,9$, one can see from the above list that $a_{i}$ is a prime element, or $\tau$ multiplied by an inverse of a prime element, and since $\tau \in \operatorname{Center}(G)$, we get the Lemma for $i=1,6,9$.

For $i \neq 1,6,9, a_{i}$ is a product of a prime element with an inverse of a prime element. Write $a_{i}=f_{i} \cdot f_{i}^{\prime}$, thus, $\left(a_{i}\right)_{t_{i}}=\left(a_{i}\right)_{t_{i}^{-1}}$ will be the product of the inverse of the 2 factors times $\tau^{2}(\tau \in \operatorname{Center}(G))$. By Lemma IV.6.2 such two factors commute. Thus,

$$
\begin{aligned}
\left(a_{i}\right)_{t_{i}}=\left(f_{i}\right)_{t_{i}}\left(f_{i}^{\prime}\right)_{t_{i}} & \\
& =\tau f_{i}^{-1} \tau\left(f_{i}^{\prime}\right)^{-1}=\tau^{2} f_{i}^{-1} f_{i}^{\prime-1}=f_{i}^{-1} f_{i}^{\prime-1}=f_{i}^{\prime-1} f_{i}^{-1}=a_{i}^{-1}
\end{aligned}
$$

The same is true for $\left(a_{i}\right)_{t_{i}^{-1}}$. 
Lemma 2. If $T_{i}$ and $T_{j}$ are adjacent and $d_{i}=a_{i}$ or $\left(a_{i}\right)_{t_{i}}$ then $\left(d_{i}\right)_{\underline{t}_{\underline{j}}^{-1}} t_{i}^{-1}=$ $d_{i}^{-1}\left(d_{i}\right)_{t_{\underline{j}}^{-1}}$ for $t_{\underline{j}}=t_{j}$ or $t_{j^{\prime}}$ or $t_{j^{\prime \prime}}$.

Proof of Lemma 2. By the above list:

$a_{i}=g_{i}$ or $g_{i}^{-1} \xi_{i}$ or $g_{i} \xi_{i}^{-1}$ or $\tau g_{i}^{-1}$.

By the above Lemma:

$\left(a_{i}\right)_{t_{i}}=\tau g_{i}^{-1}$ or $\xi_{i}^{-1} g_{i}$ or $\xi_{i} g_{i}^{-1}$ or $g_{i}$.

Thus, $d_{i}=g_{i}$ or $\tau g_{i}^{-1}$ or $\left(g_{i} \xi_{i}^{-1}\right)^{ \pm 1}\left(g_{i}^{-1} \xi_{i}\right)^{ \pm 1}$.

If $d_{i}$ is of the form $g_{i}$ or $\tau g_{i}^{-1}$ it satisfies the Lemma by the definition of prime element (axiom 2). For symmetry reasons we shall only treat the case $d_{i}=g_{i}^{-1} \xi_{i}$.

Case 1. $\quad t_{\underline{j}}=t_{j}$

$$
\begin{aligned}
\left(d_{i}\right)_{t_{j}^{-1} t_{i}^{-1}} & =\left(g_{i}^{-1} \xi_{i}\right)_{t_{j}^{-1} t_{i}^{-1}}=\left(g_{i}\right)_{t_{j}^{-1} t_{i}^{-1}} \cdot\left(\xi_{i}\right)_{t_{j}^{-1} t_{i}^{-1}} \\
& \text { By axiom } 2 \text { of the prime element }\left(g_{i}^{-1} g_{i} t_{j}^{-1}\right)^{-1} \cdot \xi_{i}^{-1} \cdot\left(\xi_{i}\right)_{t_{j}^{-1}} \\
& =\left(g_{i}^{-1}\right)_{t_{j}^{-1}} g_{i} \xi_{i}^{-1}\left(\xi_{i}\right)_{t_{j}^{-1}} \\
& \text { By Proposition IV.6.2 } \xi_{i}^{-1} g_{i}\left(g_{i}^{-1}\right)_{t_{j}^{-1}} \cdot\left(\xi_{i}\right)_{t_{j}^{-1}} \\
& =\left(g_{i}^{-1} \xi_{i}\right)^{-1}\left(g_{i}^{-1} \xi\right)_{t_{j}^{-1}}=d_{i}^{-1}\left(d_{i}\right)_{t_{j}^{-1}}
\end{aligned}
$$

Case 2. $t_{j}=t_{j^{\prime}}$

$\left(d_{i}\right)_{t_{j}^{\prime}-1}=\left(d_{i}\right)_{\left(t_{j} a_{j}\right)^{-1}}=\left(\left[a_{j}, d_{i}\right] d_{i}\right)_{t_{j}^{-1}}=\left[a_{j} d_{i}\right]_{t_{j}^{-1}} \cdot\left(d_{i}\right)_{t_{j}^{-1}}$.

By Proposition IV.6.2, Claim II.4 and by the formulas for $a_{i},\left[a_{j}, d_{i}\right]$ is a product of $\tau$. Since $\tau$ is of order $2,\left[a_{j}, d_{i}\right]=\tau^{\varepsilon} \quad \varepsilon=0,1$. Thus, $\left(d_{i}\right)_{t_{j^{\prime}}^{-1}}=$ $\tau^{\varepsilon}\left(d_{i}\right)_{t_{j}^{-1}}$. So we get the claim from case 1 when multiplying each side of the equation there by $\tau^{\varepsilon}$ to get the equation for $t_{j^{\prime}}$.

Case 3

$t_{j}=t_{j^{\prime \prime}}$

$\overline{\text { As }}$ in Case 2 we get $\left(d_{i}\right)_{t_{j}^{\prime \prime}}=\left(d_{i}\right)_{t_{j}} \tau^{\varepsilon}$ and we use Case 1 to get Case 3 .

\section{Lemma 2}


Since $T_{i}$ and $T_{j}$ are adjacent, $\left\langle t_{i}, t_{j}\right\rangle=1$. We use the above Lemma to deduce from Lemma IV.3.1 that $\left\langle d_{i} t_{i}, t_{\underline{j}}\right\rangle=1 \underline{j}=j, j^{\prime}, j^{\prime \prime}$. Since $d_{i}=a_{i}$ or $\left(a_{i}\right)_{t_{i}}$ we get $\left\langle t_{\underline{i}}, t_{\underline{j}}\right\rangle=1$. This covers all the triple relations which are induced from $\varepsilon(18)$.

Lemma 3. For $i, j$ s.t. $T_{i}$ and $T_{j}$ are disjoint or transversal, we have $\left[a_{i}, t_{j}\right]=1$ and $\left[t_{\underline{t}}, t_{\underline{j}}\right]=1$ where $\underline{i}=i$ or $i^{\prime}, \underline{j}=j$ or $j^{\prime}$.

Proof of Lemma 3. It is enough to prove $\left[a_{i}, t_{j}\right]=\left[t_{i}, t_{j}\right]=\left[t_{i^{\prime}}, t_{j}\right]=$ $\left[t_{i^{\prime}}, t_{j^{\prime}}\right]=1$.

If $T_{i}$ and $T_{j}$ are disjoint, then $\left[T_{i}, T_{j}\right]=1$. If $T_{i}$ and $T_{j}$ are transversal, then $\left[\tilde{T}_{i}, \tilde{T}_{j}\right]=1$. In any case, $\left[\tilde{T}_{i}, \tilde{T}_{j}\right]=1$ and thus, $\left[t_{i}, t_{j}\right]=1$.

Now, $a_{i}$ is a prime element with s.h.t. $t_{i}$ or a product of 2 prime elements. If $t_{j}$ is disjoint from $t_{i}$ we know that each of the prime factors in $a_{i}$ commutes with $t_{j}$ by the definition of prime element. If $t_{j}$ is transversal to $t_{i}$ then each of the prime factors in $a_{i}$ commutes with $t_{j}$ by Lemma III.3.5. In any case, $\left[a_{i}, t_{j}\right]=1$. Now, $t_{i^{\prime}}=a_{i} t_{i}, \quad\left[t_{i^{\prime}}, t_{j}\right]=\left[a_{i} t_{i}, t_{j}\right]$. Since $t_{i}$ commutes with $t_{j}$ and $a_{i}$, then $\left[t_{i^{\prime}}, t_{j}\right]=\left[a_{i}, t_{j}\right]$, which equal 1 . Now, $\left[t_{i^{\prime}}, t_{j^{\prime}}\right]=\left[a_{i} t_{i}, a_{j} t_{j}\right]$. Since $t_{j}$ commutes with $a_{i} t_{i}$ and $a_{j}$ commutes with $t_{i}$ to prove that $t_{i^{\prime}}$ commutes with $t_{j^{\prime}}$ it is enough to prove $\left[a_{i}, a_{j}\right]=1$. This follows from Proposition IV.6.2.

We use the following Lemma to show that all commutation relations in $\varepsilon(18)$ are satisfied when $t_{i}$ is replacing $E_{i}$ and $a_{i}$ is replacing $A_{i}$.

Lemma 4. Let $\tilde{Z}_{i j}^{2}$ be a braid in $B_{18}$ s.t. $\tilde{Z}_{i j}$ connects $q_{i}$ or $q_{i^{\prime}}$ with $q_{j}$ or $q_{j^{\prime}}$ where outside of 2 small discs centered at $q_{i}, q_{i^{\prime}}$ and $q_{j}, q_{j^{\prime}}$ respectively, the path goes below the real line, except when it goes above some of the pairs $q_{k}, q_{k^{\prime}}$ (for $i<k<j k \in K$ ). Assume $T_{i}$ and $T_{j}$ are disjoint or transversal. Then the relation induced by $\tilde{Z}_{i j}^{2}$ via the Van Kampen-Zariski method is mapped to 1 under $\beta$.

Proof. We cut $\tilde{Z}_{i j}$ into 2 pieces, one connects $u$ with the disc around $q_{i}$ and $q_{i^{\prime}}$ from below and the other one connects $u$ with the disc around $q_{j}$ and $q_{j^{\prime}}$ above the pairs $q_{k} q_{k^{\prime}} k \in K$. Thus the relation induced from $\tilde{Z}_{i j}^{2}$ is

$$
\left[\Gamma_{\underline{i}},\left(\prod_{k \in K} \Gamma_{k}^{-1} \Gamma_{k^{\prime}}^{-1}\right) \Gamma_{\underline{j}}\left(\prod_{k \in K} \Gamma_{k^{\prime}} \Gamma_{k}\right)\right]=1
$$


where $\Gamma_{\underline{i}}=\left(\Gamma_{i}\right) \rho_{i}^{m}$ for some $m$. Since $\left(\Gamma_{i}\right) \rho_{i}=\Gamma_{i^{\prime}},\left(\Gamma_{i^{\prime}}\right) \rho_{i}=\Gamma_{i^{\prime}} \Gamma_{i} \Gamma_{i^{\prime}}$, we know that $\Gamma_{\underline{i}} \in\left\langle\left(\Gamma_{i}, \Gamma_{i^{\prime}}\right\rangle\right.$.

It is enough to consider $\Gamma_{\underline{i}}=\Gamma_{i}$ or $\Gamma_{i^{\prime}}$ and $\Gamma_{\underline{j}}=\Gamma_{j}$ or $\Gamma_{j^{\prime}}$, since by proving that $\Gamma_{i}$ and $\Gamma_{i^{\prime}}$ commutes with $X \Gamma_{j} X^{-1}$, we can conclude that $X \Gamma_{j} X^{-1}$ commutes with every element $g$ from $\left\langle\Gamma_{i}, \Gamma_{i^{\prime}}\right\rangle$; in particular, $\Gamma_{\underline{i}}$ commutes with $X \Gamma_{j} X^{-1}$. Similarly, $\Gamma_{\underline{i}}$ commutes with $X \Gamma_{j^{\prime}} X^{-1}$. Thus, $\left[X^{-1} \Gamma_{\underline{i}} X, \Gamma_{j}\right]=\left[X^{-1} \Gamma_{\underline{i}} X, \Gamma_{j^{\prime}}\right]=1$. So, $\left[X^{-1} \Gamma_{\underline{i}} X, \Gamma_{\underline{j}}\right]=1$.

Now, $\beta\left(\Gamma_{\underline{i}}\right)=t_{i}$ or $t_{i^{\prime}}, \beta\left(\Gamma_{\underline{j}}\right)=t_{j}$ or $t_{j^{\prime}}, \beta\left(\Gamma_{k^{\prime}} \Gamma_{k}\right)=t_{k^{\prime}} \cdot t_{k}=a_{k} t_{k}^{2}$. So

$$
\beta\left(\left(\prod_{k \in K} \Gamma_{k}^{-1} \Gamma_{k^{\prime}}^{-1}\right) \Gamma_{\underline{j}}\left(\prod_{k \in K} \Gamma_{k^{\prime}} \Gamma_{k}\right)\right)=\left(\prod_{k \in K} t_{k}^{-2} a_{k}^{-1}\right) a_{j}^{\delta} t_{j}\left(\prod_{k \in K} a_{k} t_{k}^{2}\right),
$$

which is a product of squares of half-twists and prime elements. Thus, we can use Propositions IV.6.1 and IV.6.2 to rearrange the factors in the above product while multiplying the product with the appropriate $\nu^{\varepsilon}$ which is a central element to get

$$
\tau^{\varepsilon} a_{j}^{\delta} \prod_{k \in K} a_{k}^{-1}\left(a_{k}\right)_{t_{j}^{-1}} \prod_{k \in K} t_{k}^{-2}\left(t_{k}^{2}\right)_{t_{j}^{-1}} \cdot t_{j}
$$

Now,

$$
a_{k}^{-1}\left(a_{k}\right)_{t_{j}^{-1}}=\left\{\begin{array}{llc}
\left(a_{k}\right)_{t_{j}^{-1}} t_{k}^{-1} & t_{j}, t_{k} \text { adjacent } & \text { (Lemma 2) } \\
1 & t_{j}, t_{k} \text { disjoint or transversal (Lemma 3). }
\end{array}\right.
$$

Since $a_{k}$ is a product of prime elements with $\tau$ by Lemma III.2.2, $\left(a_{k}\right)_{t_{j}^{-1}} t_{h}^{-1}$ is also such a product. Thus, $a_{k}^{-1}\left(a_{k}\right)_{t_{j}^{-1}}$ is 1 or a prime element supported on $t_{j}$.

By Lemma IV.4.0 when $t_{k}$ and $t_{j}$ are adjacent, $\left(t_{k}^{-2}\right)\left(t_{k}^{2}\right)_{t_{j}^{-1}}$ is a product of $c$ with an inverse of a prime element supported on $t_{j}$. By Remark III.1.1, if $t_{k}$ and $t_{j}$ are disjoint or transveral then $t_{k}^{-2}\left(t_{k}^{2}\right)_{t_{j}^{-1}}=1$.

Thus,

$$
\begin{aligned}
\beta\left(\left(\prod_{k \in K} \Gamma_{k}^{-1} \Gamma_{k^{\prime}}^{-1}\right)\right. & \left.\left.\Gamma_{\underline{j}}\left(\prod_{k \in K} \Gamma_{k^{\prime}} \Gamma_{k^{\prime}}^{-1}\right)\right)\right) \\
& =\tau^{\varepsilon} a_{j}^{\sigma} c^{t}\left(\prod\left(\begin{array}{c}
\text { prime elements supported } \\
\text { on } t_{j} \text { or their inverse }
\end{array}\right)\right) \cdot t_{j} \\
& =c^{t} \tau^{\varepsilon}\left(t_{j^{\prime}} t_{j}^{-1}\right)^{\delta}\left(\prod\left(\begin{array}{c}
\text { prime elements supported } \\
\text { on } t_{j} \text { or their inverse }
\end{array}\right)\right) \cdot t_{j} .
\end{aligned}
$$


We have to check if this product commutes with $\beta\left(\Gamma_{\underline{i}}\right)$. Since $\Gamma_{\underline{i}}$ belongs to the subgroup generated by $\Gamma_{i}$ and $\Gamma_{i^{\prime}}$ which is the same as the subgroup generated by $E_{i}$ and $E_{i^{\prime}}, \beta\left(\Gamma_{\underline{i}}\right)$ is a product of $\beta\left(E_{i}\right) \quad\left(=t_{i}\right)$ and of $\beta\left(E_{i^{\prime}}\right)(=$ $\left.t_{i}^{\prime}\right)$. Thus it is enough to check that the above product commutes with $t_{i}$ and $t_{i^{\prime}}$.

Now, $t_{i}=\tilde{T}_{i}$ and $t_{j}=\tilde{T}_{j}$ are transversal or disjoint, thus by Remark III.1.1(d) $t_{i}, t_{i^{\prime}}$ commutes with $t_{j}, t_{j^{\prime}}$. By the definition of prime element (Axiom 3) and Lemma III.4.2, a prime element supported on $t_{j}$ commutes with $t_{i}$ and $t_{i}^{\prime}$. Finally, $c, \tau \in \operatorname{Center}\left(G_{9}\right)$, and thus all the factors in the above product commute with $t_{i}$ and $t_{i^{\prime}}$ and, therefore, the product commutes.

Lemma 4

Lemma 4 covers all degree 2 elements that appear in $\varepsilon(18)$.

We still have to check the degree 1 elements that appear in $\varepsilon(18)$.

We recall from Lemma II.2 that for $E_{i}=\left\{\begin{array}{ll}\Gamma_{i} & i \neq 2,7 \\ \Gamma_{i^{\prime}} & i=2,7\end{array}\right.$ and $A_{i}=$ $E_{i^{\prime}} E_{i}^{-1}$ we have

(1) $\Gamma_{i^{\prime}} \Gamma_{i}=E_{i^{\prime}} E_{i}=A_{i} E_{i}^{2}$.

(2) $\Gamma_{i}= \begin{cases}E_{i} & i \neq 2,7 \\ E_{i}^{-1} E_{i^{\prime}} E_{i}=E_{i}^{-1} A_{i} E_{i}^{2} & i=2,7 .\end{cases}$

(3) $\Gamma_{i^{\prime}}= \begin{cases}E_{i}=A_{i} E_{i} & i \neq 2,7 \\ E_{i} & i=2,7\end{cases}$

We have to consider each of the degree 1 elements in $\varepsilon(18)$ and to apply $\beta$ on both sides of the induced relation in order to prove that we get the same element of $G_{9}$.

We want to rewrite each side of the induced relation as a product of the following form: $\tau^{\varepsilon}$. prime elements $\cdot$ half-twists, and then use formulas for the action of $\tilde{B}_{9}$ on $G_{9}$ and Lemma 1 .

We shall only consider here one braid which is a degree 1 element. We shall consider $\tilde{Z}_{22^{\prime}(1)}$.

$\tilde{z}_{22^{\prime}(1)}=i_{1 i^{\prime}} 2 / 2^{\prime} \quad$;

$\tilde{Z}_{22^{\prime}(1)}$ implies by the RMS method the relation

$\Gamma_{2^{\prime}}=\Gamma_{1^{-1}} \Gamma_{1^{\prime}}^{-1} \Gamma_{2} \Gamma_{1^{\prime}} \Gamma_{1}$.

From (2) above, this relation is actually:

$E_{2}=E_{1}^{-2} A_{1}^{-1} E_{2}^{-1} A_{2} E_{2}^{2} A_{1} E_{1}^{2}$. 
Recall that $t_{i}=\beta\left(E_{i}\right) . a_{i}=\beta_{i}\left(A_{i}\right)$. Thus,

$$
\begin{aligned}
& \beta(\ell s)=t_{2} \\
& \beta(r s)=t_{1}^{-2} a_{1}^{-1} t_{2}^{-1} a_{2} t_{2}^{2} a_{1} t_{1}^{2}= \\
& \left.=\left(a_{1}\right)_{t_{1}^{+2}}^{-1}\left(a_{2}\right)_{t_{2} t_{1}^{+2}}\left(a_{1}\right)_{t_{1}^{-2} t_{2} t_{1}^{+2}} \cdot t_{1}^{-2} t_{2} t_{1}^{2}=\text { (using Lemma } 1\right) \\
& =a_{1}^{-1} \cdot\left(a_{2}^{-1}\right)_{t_{1}^{2}}\left(a_{1}\right)_{t_{2} t_{1}^{2}} t_{1}^{-2} t_{2} t_{1}^{2}= \\
& \text { (using Lemma III.2.1(2) applied on } a_{2} \text { and on }\left(a_{1}\right)_{t_{2}} \text { ) } \\
& =g_{1}^{-1}\left(g_{2}^{-1} \xi_{2}\right)^{-1}\left(g_{1}\right)_{t_{2}} \cdot t_{1}^{-2} t_{2} t_{1}^{2}= \\
& =g_{1}^{-1} \xi_{2}^{-1} g_{2} g_{1} g_{2}^{-1} \cdot t_{1}^{-2} t_{2} t_{1}^{2}=\text { (using }\left[g_{1}^{-1}, g_{2}\right]=\tau \text { and }\left[g_{1}^{-1}, \xi_{2}^{-1}\right]=\tau \text { ) } \\
& =\tau^{2} \cdot \xi_{2} g_{2} g_{1}^{-1} g_{1} g_{2}^{-1} \cdot t_{1}^{-2} t_{2} t_{1}^{2} \\
& =\xi_{2}^{-1} t_{1}^{-2} t_{2} t_{1}^{2} t_{2}^{-1} \cdot t_{2} \\
& =\xi_{2}^{-1} \cdot t_{1}^{-2}\left(t_{1}^{2}\right)_{t_{2}^{-1}} \cdot t_{2} \\
& =\xi_{2}^{-1} \cdot \quad \xi_{2} \quad \cdot t_{2}=t_{2} \quad \text { (Lemma IV.4.2) }
\end{aligned}
$$

Theorem V.1

Lemma V.2. $\hat{\alpha} \hat{\beta}=I_{d} \quad \hat{\beta} \tilde{\alpha}=I_{d}$

Proof. By definition of $\hat{\alpha}$ and by the formula for expressing $A_{i}$ in terms of $h_{i}$ and $\eta_{i}$ (Lemma IV.8.3), $\hat{\alpha} \hat{\beta}=I d$.

For $\hat{\beta} \hat{\alpha}$ recall that $G_{9}$ is generated by $\tilde{T}_{i}$ and $g_{i}$, i.e., by $t_{i}$ and $g_{i}$, i.e., by $\tilde{T}_{i}, a_{i}$ and $\xi_{i}$.

$\hat{\beta} \hat{\alpha}\left(t_{i}\right)=\hat{\beta} \hat{\alpha}\left(\tilde{T}_{i}\right)=\hat{\beta}\left(\tilde{E}_{i}\right)=\tilde{T}_{i}=t_{i}$.

By Proposition IV.8.3 and by $\hat{\alpha}\left(\xi_{i}\right)=\eta_{i}, \quad \hat{\alpha}\left(g_{i}\right)=h_{i}$, we get $\hat{\alpha}\left(a_{i}\right)=A_{i}$. Thus, $\hat{\beta} \hat{\alpha}\left(a_{i}\right)=\hat{\beta}\left(A_{i}\right) \stackrel{\text { by def. }}{=} a_{i}$

In Lemma IV.5.3 we have expressions for $\eta_{i}$, in terms of $E_{i}$. If we apply $\hat{\beta}$ on these expressions, we get the same expressions where $E_{i}$ is replaced by $\tilde{T}_{i}$. These expressions are exactly the expressions for $\xi_{i}$ as products of $\tilde{T}_{i}$ 's from Lemma IV.4.2. Thus, $\hat{\beta}\left(\eta_{i}\right)=\xi_{i}$.

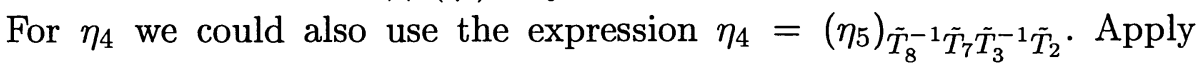
on it the $\tilde{B}_{9}$ homomorphism $\hat{\beta}$ to get $\hat{\beta}\left(\eta_{4}\right)=\left(\hat{\beta}\left(\eta_{5}\right)\right)_{\tilde{T}_{8}^{-1} \ldots \tilde{T}_{2}} \stackrel{\text { from above }}{=}$ $\left(\xi_{5}\right)_{\tilde{T}_{8}^{-1} \ldots \tilde{T}_{2}} \stackrel{\text { Lemma IV.4.2 }}{=} \xi_{4}$.

So $\hat{\beta} \hat{\alpha}\left(\xi_{i}\right)=\hat{\beta}\left(\eta_{i}\right)=\xi_{i} \quad$ by IV.4.2 and IV.5.3.

Thus, $\hat{\beta} \hat{\alpha}=I d$. 
Corollary V.3. $G \simeq \frac{\tilde{B}_{9} \ltimes G_{0}(9)}{N_{9}}=G_{9}$

Proof. $\hat{\beta}: G \rightarrow G_{9}$ is an isomorphism.

\section{Main Results and Formulations of Additional Results.}

In this chapter we shall state the main results concerning the fundamental group of the complement of a branch curve of a Veronese surface of order 3 proven in the previous chapters. The theorem is formulated in Theorem VI.1. We shall also formulate additional results on fundamental groups that were proven in earlier works as well as future results.

\section{VI.1. Main results and "forthcoming" results.}

In order to phrase the main results we recall a few definitions.

Definition. Braid group $B_{n}=B_{n}[D, K]$

Let $D$ be a closed disc in $\mathbb{R}^{2}, K \subset D, K$ finite. Let $B$ be the group of all diffeomorphisms $\beta$ of $D$ such that $\beta(K)=K,\left.\beta\right|_{\partial D}=\operatorname{Id}_{\partial D}$. For $\beta_{1}, \beta_{2} \in B$, we say that $\beta_{1}$ is equivalent to $\beta_{2}$ if $\beta_{1}$ and $\beta_{2}$ induce the same automorphism of $\pi_{1}(D-K, u)$. The quotient of $B$ by this equivalence relation is called the braid group $B_{n}[D, K](n=\# K)$. We sometimes denote by $\bar{\beta}$ the braid represented by $\beta$. The elements of $B_{n}[D, K]$ are called braids.

Definition. $H(\sigma)$, half-twist defined by $\sigma$

Let $D, K$ be as above. Let $a, b \in K, K_{a, b}=K-a-b$ and $\sigma$ be a simple path in $D-\partial D$ connecting $a$ with $b$ s.t. $\sigma \cap K=\{a, b\}$. Choose a small regular neighborhood $U$ of $\sigma$ and an orientation preserving diffeomorphism $f: \mathbb{R}^{2} \rightarrow \mathbb{C}^{1}\left(\mathbb{C}^{1}\right.$ is taken with usual "complex" orientation) such that $f(\sigma)=[-1,1], \quad f(U)=\left\{z \in \mathbb{C}^{1}|| z \mid<2\right\}$. Let $\alpha(r), r \geq 0$, be a real smooth monotone function such that $\alpha(r)=1$ for $r \in\left[0, \frac{3}{2}\right]$ and $\alpha(r)=0$ for $r \geq 2$.

Define a diffeomorphism $h: \mathbb{C}^{1} \rightarrow \mathbb{C}^{1}$ as follows. For $z \in \mathbb{C}^{1}, z=r e^{i \varphi}$ let $h(z)=r e^{i(\varphi+\alpha(r))}$. It is clear that on $\left\{z \in \mathbb{C}^{1}|| z \mid \leq \frac{3}{2}\right\}, h(z)$ is the positive rotation on $180^{\circ}$ and that $h(z)=$ Identity on $\left\{z \in \mathbb{C}^{1}|| z \mid \geq 2\right\}$, in particular on $\mathbb{C}^{1}-f(U)$. Considering $\left.\left(f \circ h \circ f^{-1}\right)\right|_{D}$ (we always take composition from left to right) we get a diffeomorphism of $D$ which switches 
$a$ and $b$ and is the identity on $D-U$. Thus it defines an element of $B_{n}[D, K]$, called the half-twist defined by $\sigma$ and denoted $H(\sigma)$.

Definition. Frame of $B_{n}[D, K]$

Let $D$ be a disc in $\mathbb{R}^{2}$. Let $K=\left\{a_{1}, \ldots, a_{n}\right\} K \subset D$. Let $\sigma_{1}, \ldots, \sigma_{n-1}$ be a system of simple paths in $D-\partial D$ such that each $\sigma_{i}$ connects $a_{i}$ with $a_{i+1}$ and for

$$
i, j \in\{1, \ldots, n-1\}, i<j \quad, \quad \sigma_{i} \cap \sigma_{j}= \begin{cases}\emptyset & \text { if }|i-j| \geq 2 \\ a_{i+1} & \text { if } j=i+1 .\end{cases}
$$

Let $H_{i}=H\left(\sigma_{i}\right)$. We call the ordered system of (positive) half-twists $\left(H_{1}, \ldots, H_{n-1}\right)$ a frame of $B_{n}[D, K]$ defined by $\left(\sigma_{1}, \ldots, \sigma_{n-1}\right)$, or a frame of $B_{n}[D, K]$ for short.

Definition. Transversal half-twists

The half-twists $H\left(\sigma_{1}\right)$ and $H\left(\sigma_{2}\right)$ will be called transversal if $C_{1}$ and $C_{2}$ intersect transversally in one point which is not an end point of either of the $\sigma_{i}$ 's.

Definition. $\tilde{B}_{n}$

Let $T_{n}$ be the subgroup of $B_{n}$ normally generated by $[X, Y]$ for $X, Y$ transversal half-twists. $\tilde{B}_{n}$ is the quotient of $B_{n}$ modulo $T_{n}$. We choose a frame $X_{i}$ of $B_{n}$. We denote their images in $\tilde{B}_{n}$ by $\tilde{X}_{i}$.

Proposition-Definition. $G_{0}(n), \tau, u_{1}$

Let $A_{n-1}$ be the free abelian group on $w_{1}, \ldots, w_{n-1} d$. Let us define a $\mathbb{Z} / 2$ skew-symmetric form on $A_{n-1}$ as follows:

$$
w_{i} \cdot w_{j}= \begin{cases}1 & (i-j)=1 \\ 0 & \text { otherwise }\end{cases}
$$

There exists a unique central extension $G_{0}(n)$, of $\mathbb{Z} / 2$ by $A_{n-1}$, with generators $u_{1} \ldots u_{n-1}$ that satisfies

$$
\begin{aligned}
& 1 \rightarrow \mathbb{Z} / 2 \stackrel{b}{\rightarrow} G_{0}(n) \stackrel{a}{\rightarrow} A_{n-1} \rightarrow 1 \\
& a\left(u_{i}\right)=w_{i} \\
& {\left[u_{i}, u_{j}\right]=b\left(w_{i} \cdot w_{j}\right)= \begin{cases}\tau & |i-j|=1 \\
0 & \text { otherwise } .\end{cases} }
\end{aligned}
$$


We always consider $G_{0}(n)$ with the standard $\tilde{B}_{n}$-action as follows:

$$
\left(u_{i}\right)_{\tilde{X}_{k}}= \begin{cases}u_{i}^{-1} \tau & k=i \\ u_{k} u_{i} & |i-k|=1 \\ u_{i} & |i-k| \geq 2\end{cases}
$$

Claim. $\operatorname{Ab}\left(G_{0}(n)\right)=A_{n-1}$ (free abelian group on $n-1$ generators), $G_{0}(n)^{\prime}=\{\tau, 1\}(\simeq \mathbb{Z} / 2)$.

Proof. Claim III.6.4.

Let $\psi_{n}$ be the standard homomorphisms $B_{n} \stackrel{\psi_{n}}{\rightarrow} S_{n}$ (= symmetric group).

Let $\mathrm{Ab}$ be the standard homomorphism $B_{n} \stackrel{\mathrm{Ab}}{\rightarrow} \mathbb{Z}$.

Since $\psi_{n}([X, Y])=1$, and $\mathrm{Ab}([X, Y])=1, \psi_{n}$ and $\mathrm{Ab}$ induce homomorphisms on $\tilde{B}_{n}$.

Definition. $\tilde{\psi}_{n}, \tilde{P}_{n}, \tilde{P}_{n, 0}, c$

$\tilde{\psi}_{n}: \tilde{B}_{n} \rightarrow S_{n}$, the induced homomorphism from $\psi_{n}$.

$\widetilde{\mathrm{Ab}}: \tilde{B}_{n} \stackrel{\widetilde{\mathrm{Ab}}}{\rightarrow} \mathbb{Z}$, the induced homomorphism from $B_{n} \stackrel{\mathrm{Ab}}{\rightarrow} \mathbb{Z}$.

$\tilde{P}_{n}=\operatorname{ker} \tilde{\psi}_{n}$.

$\tilde{P}_{n, 0}=\operatorname{ker} \tilde{\psi}_{n} \cap \operatorname{ker} \widetilde{\mathrm{Ab}}=\operatorname{ker} \tilde{P}_{n} \rightarrow \operatorname{Ab}\left(\tilde{B}_{n}\right)=\mathbb{Z}$.

$c=\left[\tilde{X}_{1}^{2}, \tilde{X}_{2}^{2}\right]$ for 2 consecutive half-twists.

Consider $\tilde{B}_{9} \ltimes G_{0}(9)$ with respect to the standard $\tilde{B}_{9}$ action on $G_{0}(9)$.

Definition. $v_{1}, N_{9}, G_{9}, \tilde{\psi}_{9}: G_{9} \rightarrow S_{9}$

$v_{1}=\left(\tilde{X}_{2} \tilde{X}_{1} \tilde{X}_{2}^{-1}\right)^{2} \tilde{X}_{2}^{-2}$ for a frame $X_{1}, \ldots, X_{8}$ of $B_{9}$.

$N_{9}=$ normal subgroup generated by $c \tau^{-1},\left(u_{1} v_{1}^{-1}\right)^{3}$.

$G_{9}=\tilde{B}_{9} \rtimes G_{0}(9) / N_{9}$.

$\tilde{\psi}_{9}: G_{9} \rightarrow S_{9} \quad \tilde{\psi}_{9}(\alpha, \beta)=\tilde{\psi}_{9}(\alpha)$.

Definition. $\quad \mathrm{Ab}_{9}, H_{9}, H_{9,0}$

$\mathrm{Ab}_{9}: G_{9} \rightarrow \mathbb{Z} \quad \mathrm{Ab}_{9}(\alpha, \beta)=\widetilde{\mathrm{Ab}}(\alpha)$.

$H_{9}=\operatorname{ker} \tilde{\psi}_{9}$.

$H_{9,0}=\operatorname{ker} \tilde{\psi}_{9} \cap$ ker $\mathrm{Ab}_{9}$.

Theorem VI.1. Let $V_{3}$ be the Veronese surface of order 3 . Let $S_{3}$ be the branch curve of a generic projection $V_{3} \rightarrow \mathbb{C P}^{2}$. Let $\mathbb{C}^{2}$ be an "affine piece" of $\mathbb{C P}^{2}$. Let $S=S_{3} \cap \mathbb{C}^{2}$. Let $G=\pi_{1}\left(\mathbb{C}^{2}-S\right)$. Then $G \cong G_{9}$ s.t. $\psi: G \rightarrow S_{9}$ is compatible with $\tilde{\psi}_{9}: G_{9} \rightarrow S_{9}$. 
Proof. Corollary V.3.

Proposition VI.2. Let $V_{3}$ be the Veronese surface of order 3 . Let $S_{3}$ be the branch curve of a generic projection $V_{3} \rightarrow \mathbb{C P}^{2}$. Let $\bar{G}=\pi_{1}\left(\mathbb{C P}^{2}-S_{3}\right)$. Consider $v_{1}$ as an element of $G_{9}$. Then there exist $w_{0} \in H_{9,0}$ s.t. $\bar{G} \simeq \bar{G}_{9}$ where $\bar{G}_{9}=G_{9} /\left\langle v_{1}^{18} w_{0}\right\rangle$.

Proof. To appear in [MoTe9].

Proposition VI.3. Let $X_{i}$ be a frame in $B_{n}$. Let $c=\left[\tilde{X}_{1}^{2}, \tilde{X}_{2}^{2}\right]$. Then

$c=\left[\tilde{X}_{1}^{2}, \tilde{X}_{2}^{2}\right]=\left[\tilde{X}_{i}^{2}, \tilde{X}_{i+1}^{2}\right]=\cdots=\left[\tilde{X}_{n-2}^{2}, \tilde{X}_{n-1}^{2}\right]$.

Moreover, $\left(\tilde{P}_{n}^{\prime}\right)=\left(\tilde{P}_{n, 0}\right)^{\prime}=\{1, c\} \simeq \mathbb{Z}_{2}$.

$\operatorname{Ab}\left(\tilde{P}_{n}\right)=$ free abelian group on $n$ generators.

$\tilde{B}_{n}$ acts on $\tilde{P}_{n, 0}$ by conjugation.

$\tilde{P}_{n, 0}$ with this action is isomorphic to $G_{0}(n)$ with the standard $\tilde{B}_{n}$-action as defined previously.

There exists a series: $1 \subseteq\left(\tilde{P}_{n, 0}\right)^{\prime} \subseteq \tilde{P}_{n, 0} \subseteq \tilde{P}_{n} \subset \tilde{B}_{n}$ s.t. $\tilde{B}_{n} / \tilde{P}_{n}=S_{n}$, $\tilde{P}_{n} / \tilde{P}_{n, 0} \simeq \mathbb{Z}, \quad \tilde{P}_{n, 0} /\left(\tilde{P}_{n, 0}\right)^{\prime} \simeq A_{n-1}=\mathrm{Ab}\left(G_{0}(n)\right), \quad\left(\tilde{P}_{n, 0}\right)^{\prime} \simeq \mathbb{Z}_{2}$.

$\operatorname{Ab}\left(B_{n}\right)=\mathbb{Z}$.

Proof. See in [MoTe4], Chapters 4, 5, definitions of $\tilde{P}_{n}$ and $\tilde{P}_{n, 0}$ and Theorem III.6.4.

Proposition VI.4. There exists a series $1 \subseteq H_{9,0}^{\prime} \subset H_{9,0} \subset H_{9} \subset G_{9}$, where $G_{9} / H_{9} \simeq S_{9}$, $H_{9,0}^{\prime}=H_{9}^{\prime}=\{1, c\} \cong \mathbb{Z} / 2$.

$H_{9} / H_{9,0} \simeq \mathbb{Z}$ $H_{9,0} / H_{9,0}^{\prime} \simeq(\mathbb{Z}+\mathbb{Z} / 3)^{8}$,

Proof. To appear in [MoTe9].

Proposition VI.5. Let $\bar{H}_{9}$ and $\bar{H}_{9,0}$ be the images of $H_{9,0}$ and $H_{q}$ in $\bar{G}_{9}$. Then $\bar{H}_{9,0}^{\prime}=\bar{H}_{q}^{\prime}$ and

$$
1 \subseteq \bar{H}_{9,0}^{\prime} \subseteq \bar{H}_{9,0} \subseteq \bar{H}_{g} \subset \bar{G}_{g}
$$

where $\bar{G}_{9} / \bar{H}_{9} \simeq S_{9}, \quad \bar{H}_{9} / \bar{H}_{9,0} \simeq \mathbb{Z}_{9}, \quad \bar{H}_{9,0} / \bar{H}_{9,0}^{\prime} \simeq(\mathbb{Z}+\mathbb{Z} / 3)^{8}, \quad \bar{H}_{9,0}^{\prime} \cong$ $\mathbb{Z} / 2$. 
Proof. To appear in [MoTe9].

\section{VI.2. The Galois cover of $V_{n}$.}

We shall quote here other results on fundamental groups related to Veronese surfaces proven in earlier works.

Theorem. Let $V_{n}$ be a Veronese surface of order $n$. Let $\left(V_{n}\right)_{\text {Gal }}$ be its Galois cover with respect to $f$, a generic projection. Then $\pi_{1}\left(\left(V_{n}\right)_{\mathrm{Gal}}^{\mathrm{Aff}}\right)$ the fundamental group of the part of $\left(V_{n}\right)_{\text {Gal }}$ that lies over a generic affine part of $\mathbb{C P}^{2}$ is a direct sum of $n^{2}-1$ cyclic groups of order $n$.

Proof. See [MoTe3].

Theorem. Let $V_{n}$ be a Veronese surface of order $n$. Let $\left(V_{n}\right)_{\mathrm{Gal}}$ be its Galois cover with respect to $f$, a generic projection to $\mathbb{C P}^{2}$. Then $\pi_{1}\left(V_{n}\right)_{\text {Gal }}$ is a direct sum of $n^{2}-2$ cyclic groups of order $n$.

Proof. See [MoTe3].

The above results concern the computation of ker $\psi$ for

$$
\psi: \frac{\pi_{1}\left(\mathbb{C}^{2}-S\right)}{\left\langle\Gamma_{j}^{2}\right\rangle} \rightarrow S_{n}
$$

for $S$, the branch curve of a generic projection to $\mathbb{C P}^{2}$ of $V_{n}$, and $\left\{\Gamma_{j}\right\}$ a $g$-base for $\pi_{1}(\mathbb{C}-S, *)$. To carry out the computation we used the relations induced from the Van Kampen method (Chapter II), $\Gamma_{j}^{2}=\Gamma_{j^{\prime}}^{2}=1$ and the $R M S$ method without using the computations of Chapter IV. These results are easier since we assume there that all generators of fundamental groups are of order 2 .

\section{VI.3. The Galois cover of $\mathrm{X}_{\mathrm{ab}}$.}

We shall also quote here a few results concerning the fundamental group of the Galois cover of $X_{a b}$.

Definition. $X_{a b}$ 
Let $X=\mathbb{C P}^{1} \times \mathbb{C P}^{1}$. Let $\ell_{1}$ be the first $\mathbb{C P}^{1}$ and $\ell_{2}$ the second one. Let $a, b \in N^{+}$. Look at $E=a \ell_{1}+b \ell_{2}$. Embed $X$ in some $\mathbb{P}^{N}$ with respect to the linear system $|E|$. Denote the image of the embedding by $X_{a b}$.

Theorem. The fundamental group $\pi_{1}\left(\left(X_{a b}\right)_{\mathrm{Gal}}^{\mathrm{Aff}}\right)$ is a finite commutative group on $n-1$ generators $(n=2 a b)$, each of order $C(c=$ g.c.d. $(a, b))$ and there are no further relations.

Proof. Theorem 10.1 from [MoTe5].

Theorem. The fundamental group $\pi_{1}\left(\left(X_{a b}\right)_{\text {Gal }}\right)$ is a finite abelian group with $n-2$ generators, each of order $c(c=$ g.c.d. $(a, b))$ and there are no further relations.

Proof. Theorem 10.2 from [MoTe5].

Corollary. If $a, b$ are relatively prime, then $\left(X_{a b}\right)_{\mathrm{Gal}}$ is simply connected.

These results give us very interesting examples of surfaces of general type. The Galois covers are minimal surfaces of general type. Their index is zero for $a=b=5$ or for $a=4$, and $b=7$ and positive for $a \geq 5, b \geq 6$. By the above Corollary, they are simply connected for $a, b$ relatively prime. Thus we get a series of simply connected surfaces of general type with positive index unlike the Bogomolov-watershed Conjecture (see [FH]). Moreover, $X_{55}$ is an example of a surface of general type with zero index and even type with finite commutative fundamental group whose universal cover is homomorphic to a connected sum of $S^{2} \times S^{2}$. $X_{55}$ gives also an exotic differential structure on a connected sum of several copies of $S^{2} \times S^{2}$. There are only a few other such examples (one of them is $X_{4,7}$ ). The other 3 examples will appear in [MoTe10].

In this work we have computed fundamental groups of complements of branch curves as part of our research on algebraic surfaces. This work also has implications to the topology of complements of curves in general. For general singular curves see, for example, [L1] and [L2].

\section{References.}

[A] E. Artin, Theory of braids, Ann. Math. 48 (1947), 101-126. 
[B] J. Birman, Braids, Links and Mapping Class Groups, Princeton University Press, 1975.

[C1] F. Catanese, On the moduli spaces of surfaces of general type, J. Diff. Geom. 19, 2 (1984), 483-515.

[C2] F. Catanese, Connected components of moduli spaces, J. Diff. Geom. 24 (1986), 395-399.

[FH] Feustel / Holzapfel, Symmetry points and Chern invariants of Picardmodular-surfaces, Math. Nach. 111 (1983), 7-40.

[L1] A. Libgober, Fundamental groups to complements to plane singular curves, Proc. of the AMS, 46 (1988), 28-45.

[L2] A. Libgober, Homotopy groups of the complements to singular hypersurfaces II, Ann. of Math. 139 (1994), 119-145.

[Mo] B. Moishezon, Stable branch curves and braid monodromies, in Lect. Notes in Math. 862 (1981), 107-192.

[Mo1] B. Moishezon, Algebraic surfaces and the arithmetic of braids, I, in Arithmetic and Geometry, papers dedicated to I.R. Shafarevich, Birkhäuser, 1983, 199-269.

[Mo2] B. Moishezon, Algebraic surfaces and the arithmetic of braids, II, Contemp. Math. 44 (311-344), 1985.

[MoTe1] B. Moishezon and M. Teicher, Existence of simply connected algebraic surfaces of positive and zero indices, Proceedings of the National Academy of Sciences, United States of America, 83 (1986), 6665-6666.

[MoTe2] B. Moishezon and M. Teicher, Simply connected algebraic surfaces of positive index, Invent. Math. 89 (1987), 601-643.

[MoTe3] B. Moishezon and M. Teicher, Galois coverings in the theory of algebraic surfaces, Proc. of Symp. in Pure Math. 46 (1987), 47-65.

[MoTe4] B. Moishezon and M. Teicher, Braid group technique in complex geometry, I, Line arrangements in $\mathbb{C P}^{2}$, Contemp. Math. 78 (1988), 425-555.

[MoTe5] B. Moishezon and M. Teicher, Finite fundamental groups, free over $\mathbb{Z} / c \mathbb{Z}$, for Galois covers of $\mathbb{C P}^{2}$, Math. Ann. 293 (1992), 749-766.

[MoTe6] B. Moishezon and M. Teicher, Braid group technique in complex geometry, II, From arrangements of lines and conics to cuspidal curves, in Algebraic Geometry, Lecture Notes in Math. 1479 (1990). 
[MoTe7] B. Moishezon and M. Teicher, Braid group techniques in complex geometry III, Projective degeneration of $V_{3}$, Contemp. Math. 162, 313-332.

[MoTe8] B. Moishezon and M. Teicher, Braid group techniques in complex geometry IV, Braid monodromy of the branch curve $S_{3}$ of $V_{3} \rightarrow \mathbb{C P}^{2}$ and application to $\pi_{1}\left(\mathbb{C P}^{2}-S_{3}, *\right)$, Contemp. Math. 162, 332-358.

[MoTe9] B. Moishezon and M. Teicher, Fundamental groups of complements of curves in $\mathbb{C P}^{2}$ as solvable groups, in preparation.

[MoTe10] B. Moishezon and M. Teicher, On the Galois cover of Hirzebruch surfaces, in preparation.

[MoTe11] B. Moishezon and M. Teicher, New spin manifolds, in preparation.

[MoTe12] B. Moishezon and M. Teicher, Singularities and Algebraic Surfaces, to appear, Academic Press.

[P] U. Persson, Chern invariants of surfaces of general type, Comp. Math. 43 (1981), 3-58.

[VK] E.R. Van Kampen, On the fundamental group of an algebraic curve, Am. J. Math. 55 (1933), 255-260.

[Z] O. Zariski, Algebraic Surfaces (Ch. VIII), Second Edition, Springer 1971.

ReCEIVEd April 5TH, 1994.

DEPT. OF MATH.

Columbia UNIVERSiTy

NEW YoRK, N.Y. 10027

U.S.A.

AND

Dept. of Math. \& Comp. Sci.

BAR-IlaN UNIVERSITY

RAMAT-GAN 52900

ISRAEL 